Dissertation zur Erlangung des Doktorgrades der

Fakultät für Chemie und Pharmazie

der Ludwig-Maximilians-Universität München

\title{
Firefly Luciferase Mutants as Sensors of Proteome Stress
}

Rajat Gupta

aus

New Delhi

India

2012 


\section{Erklärung}

Diese Dissertation wurde im Sinne von $§ 7$ der Promotionsordnung vom 28. November 2011 von Herrn Prof. Dr. F. Ulrich Hartl betreut.

\section{Eidesstattliche Versicherung}

Diese Dissertation wurde eigenständig und ohne unerlaubte Hilfe erarbeitet.

München, am 18.09.2012

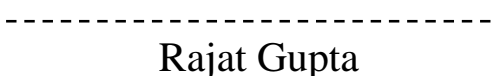

Rajat Gupta

Dissertation eingereicht am: 18.09 .2012

1. Gutachter: $\quad$ Prof. Dr. F. Ulrich Hartl

2. Gutachter: PD Dr. Konstanze Winklhofer

Mündliche Prüfung am: $\quad 06.11 .2012$ 


\section{Acknowledgements}

I would like to express my deepest gratitude to Prof. Dr. F. Ulrich Hartl for giving me an opportunity to work in his international and interdisciplinary team. His constant support, faith and encouragement have been extremely helpful for the development of the work presented in this thesis. The stimulating discussions and exchange of ideas with Prof. Hartl helped me to nurture the scientific and analytical skills. I also like to thank Dr. Manajit Hayer-Hartl for her constant advice and suggestions during the course of the work.

I would like to thank PD Dr. Konstanze Winklhofer for being a co-referee of my thesis committee.

I am grateful to Dr. Mark Hipp for his constant support and suggestions for the development of this project and for his help in my thesis corrections.

I would also like to thank Dr. Niti Kumar, Dr. Frédéric Frottin and Dr. Prasad Kasturi for their immense help during scientific discussions and correction of this thesis and for their constant encouragement. I sincerely acknowledge Dr. Prasad Kasturi for performing the crucial experiments in C. elegans. Many thanks to Dr. Andreas Bracher and Dr. Swasti Raychaudhuri for their contributions in the work presented in this thesis.

I am also grateful to Evelyn, Silke and Andrea for their kind help in all my administrative-related queries and to Nadine, Emmanuel, Elisabeth, Verena, Romy and Albert for their expert technical and logistical help. Without their support the journey would have been difficult.

I would like to take this opportunity to express my sincere thanks to Dr. Niti kumar, Dr. Sathish Lakshmipathy, Dr. Rashmi Gupta, Dr. Jyoti Sinha, Christian Löw, Andreas Wörner, Florian Rüßmann, Matthias Antonin, Dr. Frédéric Frottin, Dr. Julian Micoud, Ayano Konagai, Yury Kukushkin and rest of the Hartl department for all the endless fun in the lab. All these people have significantly contributed in smoothing my stay in Germany and without their support and fun my Ph.D. journey would have been incomplete.

Lastly, I am indebted to my family members whose constant faith and motivation has allowed me to reach this stage where I can express my gratitude to all the people associated with me in these last four years. The work presented in this thesis is accredited to my family members for their unconditional love. 


\section{Contents}

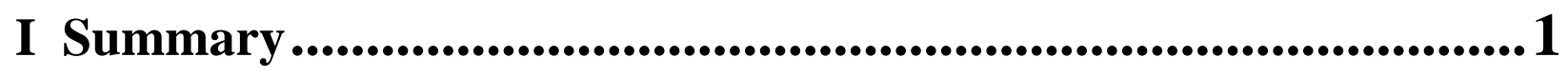

II Introduction ...............................................................................4

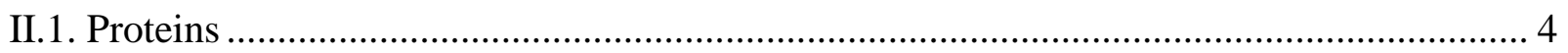



II.3. Energy Landscape Perspective on Protein Folding ............................................................. 6

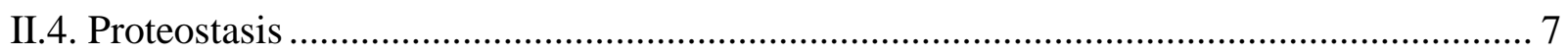



II.4.1.1. Different Classes of Chaperones ....................................................................... 11

II.4.1.1.1. Ribosome Associated Chaperones................................................................ 12

II.4.1.1.2. Non-ribosome Associated Chaperones......................................................... 13

II.4.1.1.2.1. The Hsp70 Chaperone System .............................................................. 14

II.4.1.1.2.2. The Hsp90 Chaperone System ............................................................... 19

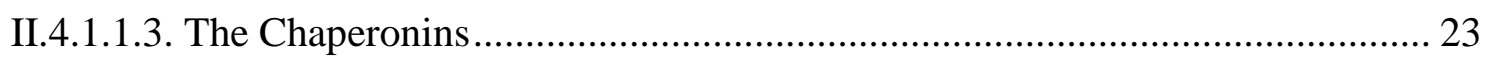

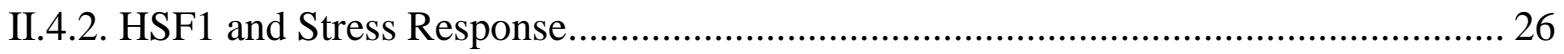



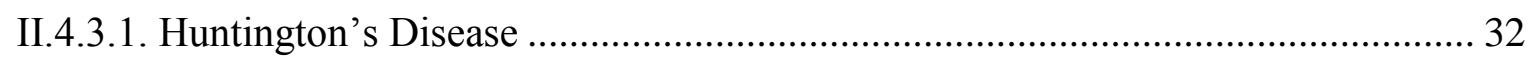

II.4.4. Cellular Defense Mechanisms against Protein Misfolding ....................................... 34

II.4.4.1. Chaperone Mediated Refolding and Disaggregation .......................................... 34

II.4.4.2. The Degradation Arm of Proteostasis ................................................................... 36

II.4.4.2.1. The Ubiquitin-Proteasome System (UPS) .................................................... 37

II.4.4.2.2. Autophagy Mediated Degradation................................................................. 40

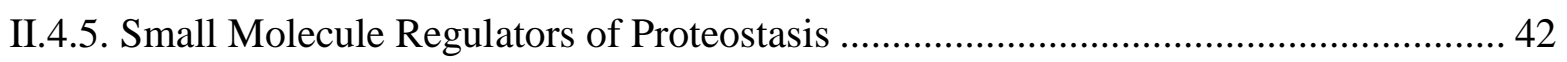

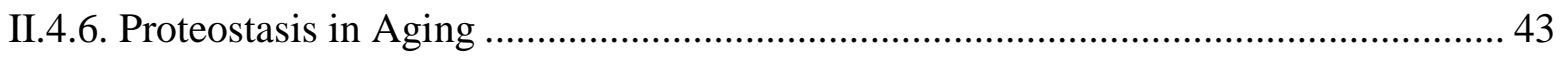

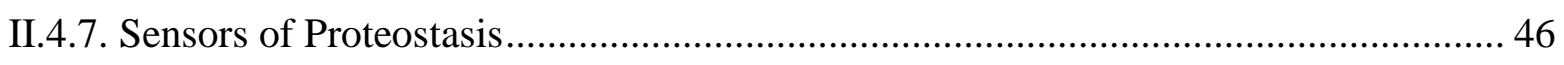

III Aim of the Study .................................................................. 48

IV Materials and Methods .....................................................49

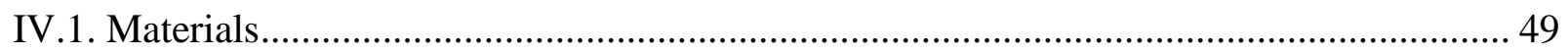




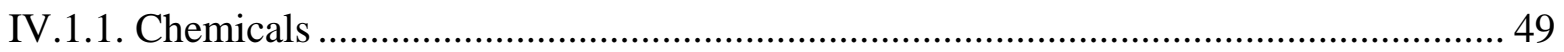

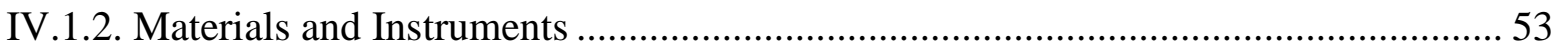

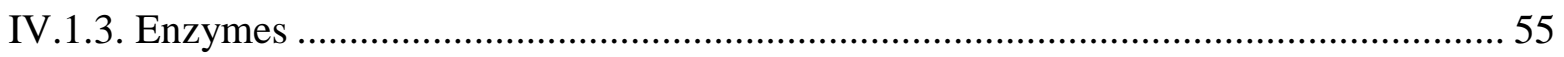

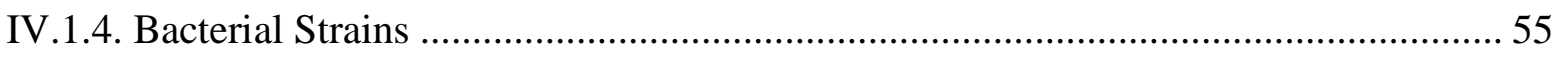



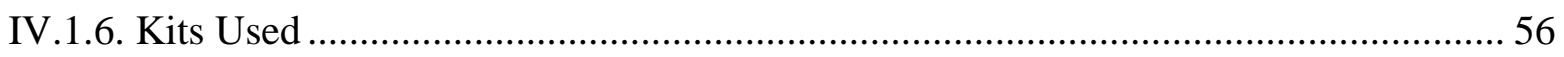

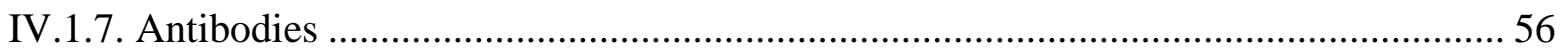

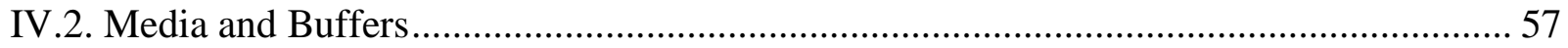

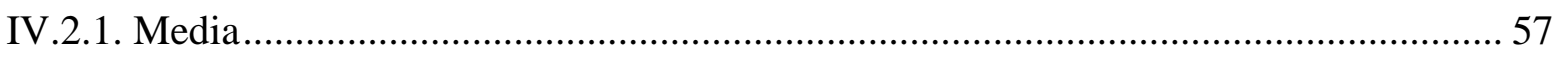

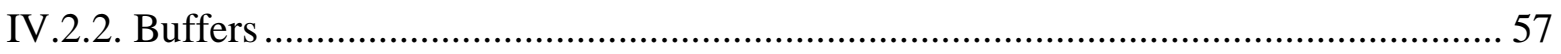

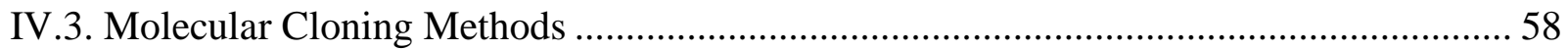

IV.3.1. Preparation and Transformation of Chemically Competent E. coli Cells ................. 59



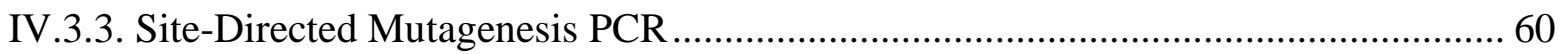



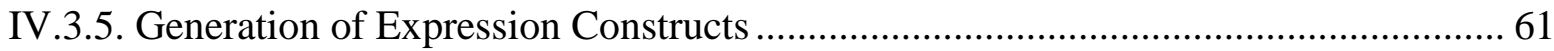

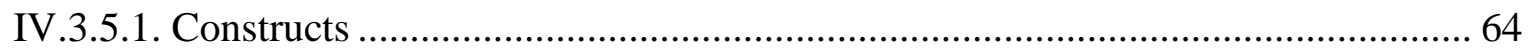

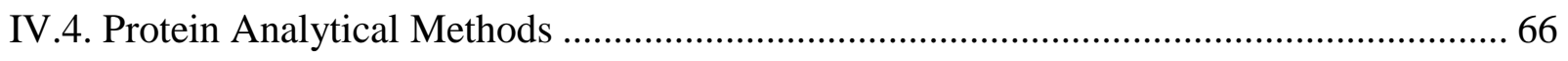

IV.4.1. Sodium Dodecyl Sulfate-Polyacrylamide Gel Electrophoresis (SDS-PAGE).......... 66

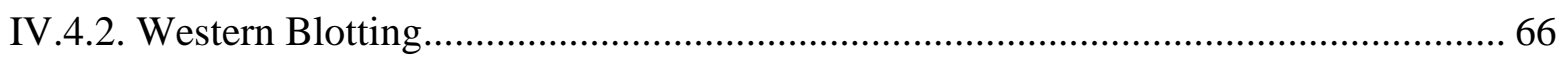



IV.5. In Vitro Characterization of Fluc Mutants ..................................................................... 67

IV.5.1. Thermal Denaturation of Fluc Variants in RRL..................................................... 67

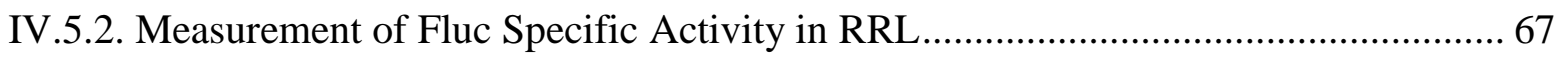

IV.5.3. Assessment of Co-translational Folding Efficiency of Fluc Variants in RRL .......... 68

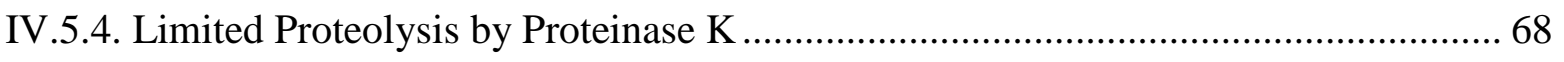

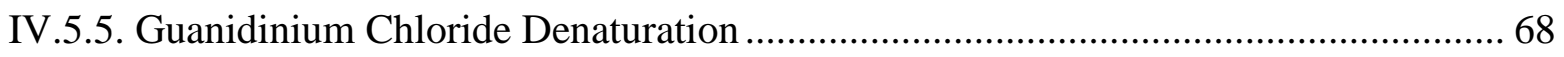

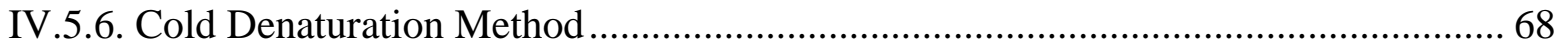

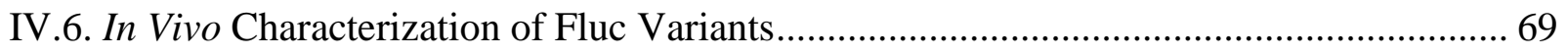

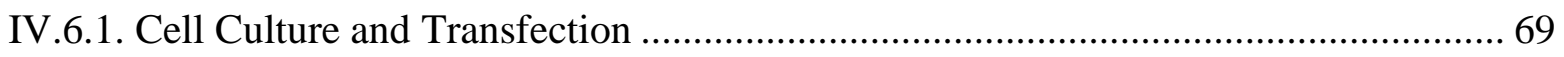

IV.6.2. Protein Sample Preparation and Specific Activity Measurements............................ 70 
IV .6.3. Fluorescence Microscopy …………………............................................................. 70

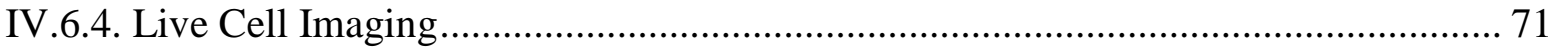

IV.6.5. Generation of Stable Cell Lines ......................................................................... 72

IV.6.6. Cryo-Preservation of Mammalian Cell Lines ............................................................. 73

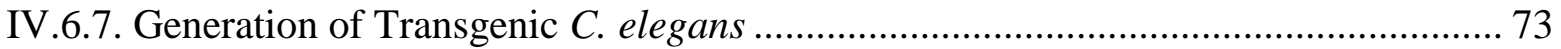

IV.6.8. Reverse Transcriptase-PCR (RT-PCR) Experiments in C. elegans .......................... 74

V Results .........................................................................................75

V.1. Generation of Conformationally Destabilized Firefly Luciferase Mutants....................... 75

V.2. In Vitro Characterization of Fluc Mutants ......................................................................... 77

V.2.1. Functional Characterization of Fluc Mutants in RRL …………………................... 77

V.2.1.1. Thermal Instability of Fluc Mutants .................................................................... 77

V.2.1.2. Specific Activities of Fluc Mutants in RRL.................................................... 80

V.2.1.3. Co-translational Folding Efficiency of Fluc Variants........................................... 82

V.2.2. Structural Characterization of Fluc Variants in RRL ................................................. 83

V.2.2.1. Sensitivity of Fluc Variants to Proteinase K ..................................................... 83

V.2.2.2. Sensitivity of Fluc Variants to Guanidinium Chloride Mediated Denaturation .. 84

V.2.2.3. Sensitivity of Fluc Variants to Cold Denaturation............................................... 85

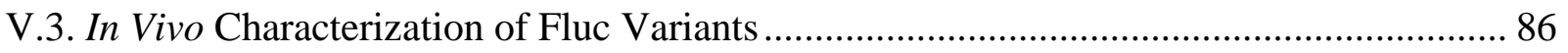

V.3.1. Chaperone Dependence of Fluc Variants in vivo ........................................................ 86

V.3.2. Generation and Characterization of Fluc-EGFP Variants in RRL ............................. 87

V.3.3. Thermal Stability of Fluc-EGFP Variants in vivo ...................................................... 89

V.3.4. Effect of Fluc-EGFP-based Sensors on the Cytosolic Stress Response ..................... 92

V.4. Applications of Fluc-EGFP-based Sensors in vivo ………............................................. 95

V.4.1. Assessment of Proteostasis Capacity in Presence of Small Molecule Inhibitors ....... 95

V.4.2. Analysis of Proteostasis Collapse by Huntingtin Protein .............................................. 97

V.4.3. Assessing Proteostasis in C. elegans Upon Heat Stress ............................................ 101

V.4.4. Assessing Proteostasis in C. elegans During Aging ................................................... 104

V.4.5. Analysis of the Proteostasis Capacity of Cytosol and Nucleus ............................... 105

V.4.5.1. Design and Biochemical Characterization of NLS- and NES-Fluc-EGFP Variants 105 
V.4.5.2. Effect of Proteasome Inhibition on the Stability of Compartment Specific Fluc Sensors 109

V.4.5.3. Assessment of the Recovery Capacity of Nucleus and Cytosol after MG132 Removal....

V.4.5.4. Role of the Nucleolus in Stress Response

V.4.5.5. The Aggregates of Fluc-EGFP Variants Co-localize with 20S Proteasome and Ubiquitin upon Proteasome Inhibition 116

V.4.5.6. Role of the Nucleus in the Degradation of NES-FlucDM-EGFP . 118

V.4.5.7. Effect of DnaJB1 Down-regulation on the Degradation of NES-FlucDM-EGFP

V.4.5.8. Effect of DnaJB1 Down-regulation on the Subcellular Localization of NES-

FlucDM-EGFP Aggregates upon Proteasome Inhibition 121

V.4.5.9. Effect of a Pharmacological Chaperone on the Stability of NLS- and NES-FlucEGFP Variants

\section{Discussion} 125

VI.1. Fluc Mutants Are Structurally and Functionally Destabilized.... 127

VI.2. Assessment of Folding Capacity of Cells Using Fluc-Based Sensors ..... 129

VI.3. Assessment of Refolding Capacity of Cells by Fluc Sensors 131

VI.4. Fluc-Based Sensors Report on Tissue-Specific Differences in C. elegans.... 132

VI.5. Fluc-Based Sensors Report on Proteostasis Collapse by Mutant Huntingtin 133

VI.6. Analysis of Intra-Compartmental Proteostasis Capacity by Fluc-Based Sensors........ 135

VI.7. Role of the Nucleus in Protein Quality Control of Cytoplasmic Proteins 137

VI.8. Role of DnaJB1 in the Degradation of Proteins 138

\section{References}

VIII Appendices.........................................................................................172

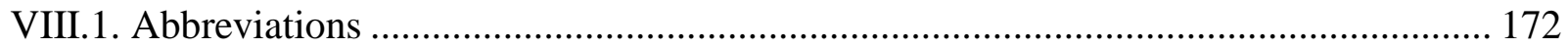

VIII.2. Curriculum Vitae ......................................................................................... 175 


\section{Summary}

Proteostasis is defined as the ability of cells to control the concentration, conformation and subcellular location of its proteins by maintaining a dynamic equilibrium between protein synthesis and degradation. All cellular factors that participate and assist in maintaining protein homeostasis under normal and stress conditions constitute the proteostasis network (PN). Molecular chaperones and components of the degradation machinery form central hubs of the PN and play a pivotal role in the life of a protein from its synthesis to degradation. Deficiencies in proteostasis are associated with numerous diseases, such as neurodegenerative diseases (Huntington's disease, Parkinson's disease etc.), cancer and cardiovascular disease. Additionally, the gradual loss of cellular proteostasis capacity is implicated both as a cause and consequence of the aging process.

The understanding of proteostasis and its regulation requires a suitable sensor that can report on the status of the PN under different cellular conditions. Previously reported proteostasis sensors were either tissue-system specific or limited to measuring the changes in specific components of the PN. Thus, they cannot be used to report global changes in the PN in different cell types and organs. To circumvent all these limitations, we developed temperature sensitive mutants of the enzyme Firefly luciferase (Fluc) as proteostasis sensors.

We selected Fluc because it provides several advantages: A.) The enzyme has no biological role in widely used cellular and animal systems, and thus the sensors derived from it can be used with minimal perturbation of the system under investigation. B.) Fluc depends on chaperones for its folding and therefore the protein folding and refolding capacity of cells can be readily estimated via enzymatic activity measurements. C.) Fluc activity can be measured by a simple luminescence-based assay with exquisite sensitivity and a wide dynamic range in cell extracts, intact cells and model organisms. Moreover, by tagging Fluc with appropriate signal sequences, inter-compartmental proteostasis can be easily studied.

In this study, we generated 6 single and 12 Fluc double mutants by weakening polar contacts in the Fluc three-dimensional structure. Using translation in rabbit reticulocyte lysate (RRL), we showed that the mutations affected the thermostability of the variants to different 
degrees. Among the 18 Fluc variants, we selected the least stable single mutant, R188Q (FlucSM) and the least stable double mutant, R188Q+R261Q (FlucDM) for further in vitro and in vivo characterization. We probed the effect of mutations on the structure of Fluc variants by several assays such as proteinase $\mathrm{K}$ digestion and could show that FlucSM and FlucDM are conformationally destabilized.

To further increase their potential as biological sensors, we tagged Fluc variants with EGFP. We were able to show that Fluc-EGFP based sensors do not significantly affect the proteostasis capacity of the cellular system under study. We next demonstrated the applications of Fluc-EGFP variants in different cell types and model organism under various stress conditions. Upon heat stress, FlucSM and FlucDM formed aggregates in HeLa cells which were efficiently resolubilized during recovery. Thus, Fluc-based sensors function as reporters of heat stress and allow assessment of the cellular capacity to refold heat denatured protein. We also showed that Fluc-EGFP based sensors are efficient in reporting the global changes in proteostasis when folding and degradation components of the PN are inhibited by small molecule inhibitors. Next we demonstrated that the Fluc-based sensors are efficient in reporting the decline in cellular proteostasis capacity by mutant huntingtin with an expanded polyglutamine stretch. Using C. elegans, we demonstrated the application of Fluc-EGFP variants in studying tissuespecific differences upon heat stress and during aging. We showed that body-wall muscle cells and neuronal cells in C. elegans respond differentially to acute stress (heat stress) and to chronic proteome stress during aging.

We tagged Fluc-EGFP and FlucDM-EGFP with a nuclear localization signal (NLS) or a nuclear export signal (NES) to study inter-compartmental proteostasis between the nucleus and the cytoplasm. Using Fluc-EGFP variants, we showed that although the nucleus is highly sensitive to proteostasis imbalance, it is more efficient in recovering from stress than the cytosol. Furthermore, contrary to our expectations, we observed that NES-FlucDM-EGFP formed aggregates in the nucleus upon proteasome inhibition. These nuclear aggregates were present in the nucleoli and they co-localized with $20 \mathrm{~S}$ proteasomes and ubiquitin. We showed that the degradation of NES-FlucDM-EGFP is enhanced when it is retained in the nucleus, suggesting a role of the nucleus in degradation. We also proposed a role of DnaJB1 (Hsp40), a co-chaperone of Hsp70, in targeting and degradation of cytosolic misfolded protein in the nucleus. 
In summary, this study describes the development and characterization of Fluc-EGFP based sensors and their potential applications to assess the proteostasis status in a wide range of experimental systems, including cell and organism models of stress, neurodegenerative disease and aging. The use of the sensors to understand the differential regulation of the PN in the nucleus and the cytosol is also described. 


\section{Introduction}

\section{II.1. Proteins}

Cells are the basic and elementary entities of an organism and contain a wide range of biomolecules such as nucleic acids, proteins, carbohydrates and lipids to constitute their structural and functional framework. The genetic information encoded by nucleic acids is transcribed and translated into proteins which are highly specialized in their functions. Protein molecules are polymers of a combination of 20 different L-amino acids which have the same backbone but different side chains. It is the sequence and combination of amino acids that confers different physical, chemical and biological properties to proteins and allows them to adopt a unique three-dimensional structure that reflects their functions inside the cell.

There are four different hierarchical levels of organization of protein structure. The first level is primary structure, which is the linear sequence of amino acids translated from the genetic code. The next level is secondary structure which refers to the spatial arrangement of amino acids in a particular order giving rise to a highly regular local sub-structure. Generally, $\alpha$-helix, $\beta$-sheet and $\beta$-turns are the most common forms of secondary structures in proteins. The third level refers to the tertiary structure which involves the global positioning of the secondary structure elements in three-dimensional space and both covalent (peptide bonds and disulfide bridges) and non-covalent interactions (hydrogen bonds, Van-der-Waals and hydrophobic interactions) are the determinants of this type of arrangement. The last level of protein organization is quaternary structure which results from the assembly of two or more separate polypeptide chains (identical or different) in three-dimensional complexes to form a multisubunit protein.

\section{II.2. Protein Folding}

Synthesis of proteins begins on the ribosome, a giant multi-molecular complex of proteins and ribosomal RNA that, in concert with other protein molecules, translates the genetic code of the messenger RNA (mRNA) into a linear chain of amino acids called polypeptide. The amino acids are covalently linked to each other by a peptide bond between carboxyl and amino groups, a reaction catalyzed by ribosomes during translation. This process of translation takes place in a vectorial fashion with the N-terminus of polypeptide emerging first from the 
ribosome's exit tunnel. The newly synthesized polypeptide chain must acquire a unique threedimensional conformation in order to perform its function in the cell. This conformation is referred as "native state" of a protein and the process by which the polypeptide chains adopt their functional structure is called "protein folding".

During protein folding, a polypeptide chain in solution can access a large number of conformations because of the free rotation of every single covalent bond in the backbone of its constituting amino acids. If a polypeptide has to scan all the possible available conformations it would take an astronomical amount of time to reach its native state. This ambiguity between the calculated time and the actual time that a polypeptide takes to fold is known as the Levinthal Paradox (Levinthal et al., 1962; Zwanzig et al., 1992). Since in cells protein folding usually occurs on a biologically relevant time scale, in the order of seconds to minutes, Levinthal proposed that folding follows a well-defined pathway, taking place through a series of transient intermediates rather than through a random conformational search.

Several models have been proposed to account for the rapid folding of proteins. According to the first model called "hydrophobic collapse" model, there is a rapid non-specific collapse of the polypeptide chain where hydrophobic non-polar side chains are buried deep inside the core, and hydrophilic and polar side chains are exposed on the surface of the folded protein. From a thermodynamic perspective, the sequestration of the hydrophobic side chains from the solvent facilitates the formation of folding intermediates and therefore hydrophobic collapse occurs spontaneously and results in the formation of the molten globule state (Dill et al., 1995; Kauzmann, 1959). The second view about protein folding, also regarded as "framework model" postulates that the folding process is a directed process where the local interactions lead to the transient formation of the secondary structural elements. Once a framework is set, the secondary structures then eventually collapse to give rise to the tertiary structure of a protein (Baldwin, 1989; Fersht, 2008; Kim and Baldwin, 1982). To reasonably account the folding process, a third view called "nucleation-condensation model" unifies the features of both the hydrophobic collapse model and the framework model. According to this model, folding is a hierarchical process that begins with the initial rapid formation of the secondary structures. There is a gradual build-up/layering of new structures over the previous ones which help in stabilization of folding intermediates. During this process, the water molecules are excluded 
from the hydrophobic core which minimizes the free energy and leads to the collapse of the partially folded polypeptide chain to its native state (Daggett and Fersht, 2003; Dill et al., 1995).

\section{II.3. Energy Landscape Perspective on Protein Folding}

In the past, many models were proposed to describe protein folding in vitro. However, the complexity of the folding process and its variability for different proteins delayed the understanding of this dynamic process. Moreover, the initial models mainly focused on folding of small single domain proteins upon dilution from denaturant. To overcome the short comings of these models, a different perspective based on the protein's potential energy surface was developed. This energy landscape perspective, also referred as "folding funnel" is a pictorial representation that describes the progression of an unfolded polypeptide chain with a very highdimensional accessible conformational space en route to the native state at the global energy minimum (Bryngelson et al., 1995; Clark, 2004; Dill and Chan, 1997) (Figure 1).

The folding funnel can often be rugged due to kinetic traps where partially folded intermediates get entrapped. These kinetic traps, also called local energy minima, arise due to non-specific intramolecular interactions in the folding intermediates (Brockwell and Radford, 2007). The number and depth of local kinetic traps on the energy landscape defines the degree of ruggedness of the folding pathway (Onuchic et al., 1997). Under physiological conditions, the folding of nascent chains becomes even more complex due to the collision between different folding intermediates. Such intermolecular interactions besides impairing the folding process can also lead to the entrapment of partially folded or misfolded states in the major kinetic traps where they may eventually aggregate. These aggregates including amyloid fibrils have very deep global energy minima and therefore they are more stable species than the native protein itself. Cells have evolved complex mechanisms to prevent these off-pathway aggregation reactions by encoding a set of helper proteins called molecular chaperones that prevent non-native interactions between the folding intermediates and help in smoothing the energy landscape (Hartl et al., 2011). 


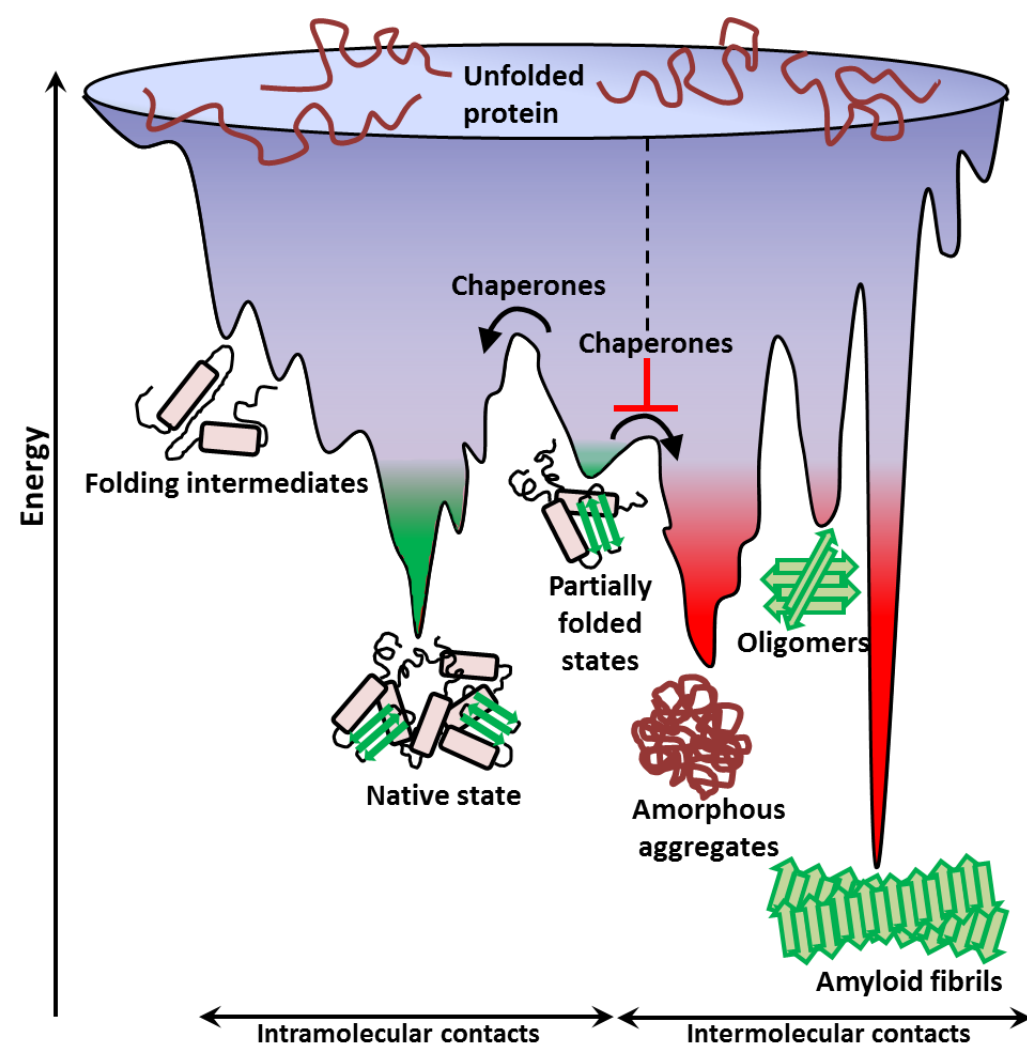

\section{Figure1: Energy landscape perspective on protein folding and misfolding.}

Scheme of a funnel-shaped folding pathway that polypeptide chains undergo in order to reach their native state by forming intramolecular contacts. The polypeptide chains at the top of funnel can acquire a wide range of conformations and as they move down the funnel to reach their native state (shown in green), their folding intermediates can get kinetically trapped. In vivo, molecular chaperones help these folding intermediates in traversing the rugged energy-landscape by preventing intermolecular interactions. However, due to molecular crowding, several folding intermediate species may engage in non-specific intermolecular interactions to form amorphous aggregates, oligomers or amyloid fibrils. These products of off-pathway folding reaction have very deep global energy minima (hence they are highly stable) and therefore they might get trapped irreversibly (shown in red). Modified from (Hartl et al., 2011).

\section{II.4. Proteostasis}

Protein folding in cells is a highly complex and challenging process due to the highly crowded and heterogeneous intracellular environment. Escherichia coli express around 300-400 $\mathrm{gl}^{-1}$ of total macromolecules in the cytoplasm (Zimmerman and Trach, 1991). It has been estimated that macromolecules occupy around $20-30 \%$ of the total cell's volume and therefore this fraction is physically unavailable to other molecules. This results in exclusion of volume that a molecule can occupy and causes crowding (van den Berg et al., 2000). Macromolecular crowding also plays a role in the functional interactions between molecules (Martin and Hartl, 
1997). However, crowding can also considerably enhance the affinities between protein molecules, especially partially folded intermediates. Aberrant non-specific interactions between non-native structures can lead to the formation of aggregates (Dobson, 2003; Ellis, 2006). Furthermore, in such a crowded milieu, these interactions are significantly aggravated upon cellular stress. The source of the stress can be environmental such as heat stress or intrinsic such as oxidative stress.

Aggregates, often exposing 'sticky' hydrophobic residues can potentially sequester essential cellular factors leading to cellular toxicity and numerous protein conformational disorders, such as Alzheimer's disease, Parkinson's disease, amyotrophic lateral sclerosis, Huntington's disease and prion diseases (Bucciantini et al., 2002; Olzscha et al., 2011; Suhr et al., 2001). To circumvent the unproductive folding of nascent chains and to prevent misfolding and aggregation of proteins, cells inherently protect their proteome through protein quality control at various stages from protein synthesis to folding and degradation of irreversibly misfolded species. Thus, cells have evolved the ability to adapt to non-permissive conditions by up-regulating the expression of a subset of proteins that participate in restoring the disturbed homeostasis.

The capacity of cells to control the concentration, conformation, binding interactions and subcellular location of each individual protein constituting the proteome is called protein homeostasis or proteostasis (Balch et al., 2008; Powers et al., 2009). The set of factors/proteins that engage in maintaining the health of other proteins from their birth to their death constitute the proteostasis network (Figure 2). Among these, molecular chaperones and components of the cellular degradation machinery, namely the ubiquitin proteasome system and autophagy form the central hub of the proteostasis network (PN). Protein remodeling factors comprising of proteins engaged in refolding misfolded proteins, disaggregation and translocation across biological membranes are equally important for the maintenance of cellular proteostasis. A deficiency in proteostasis has been implicated in numerous human diseases and contributes to cellular dysfunction and aging. 


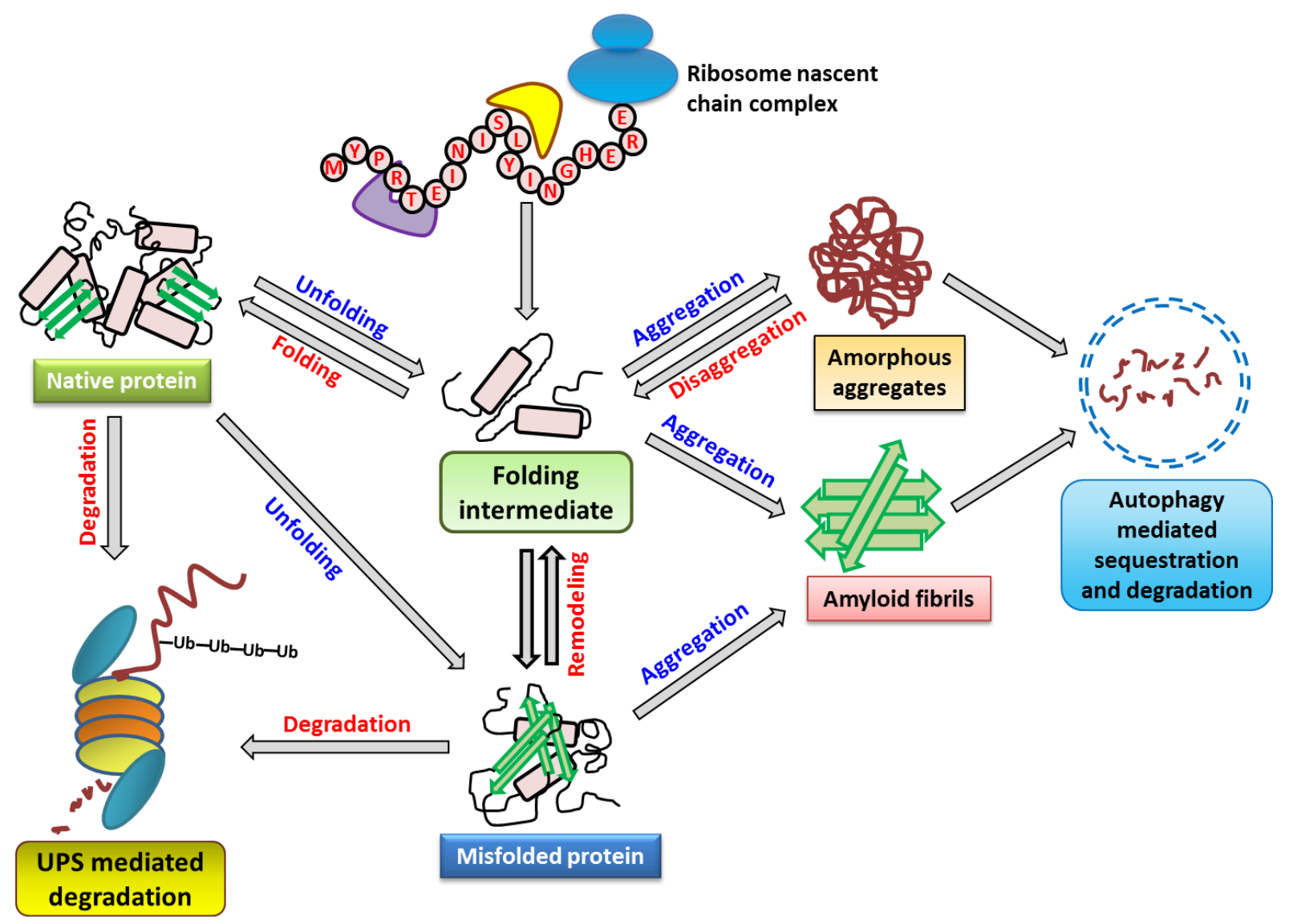

Figure 2: The major components of the proteostasis network (PN).

The PN comprises over 1000 general and specialized components such as chaperones, components of degradation machinery (ubiquitin, proteasome and autophagy) and proteins involved in trafficking that integrate with each other to maintain cellular protein homeostasis. Chaperones shield the hydrophobic patches on emerging nascent chains and aid the folding intermediates to reach their native state. Intermolecular interactions between partially folded intermediates result in protein misfolding. The misfolded proteins are either refolded to the native state by chaperones or they are eventually degraded by the ubiquitin proteasome system. Failure in refolding and degradation results in the accumulation of misfolded proteins and the formation of aggregates. The aggregates can be resolubilized by the action of disaggregase or they are cleared by autophagy mediated sequestration and degradation.

\section{II.4.1. Molecular Chaperones in Proteostasis}

Competition between protein folding and misfolding is a fundamental feature of the folding process in cells. During translation, nascent chains are inserted into the crowded milieu of the cell and expose numerous hydrophobic surfaces and regions of unstructured polypeptide backbone that can mediate non-covalent interactions with other non-native polypeptides. Bacteria have fast translation rates of 10-20 residues per second compared to $\sim 3-8$ residues in 
eukaryotes (Liang et al., 2000; Pedersen, 1984). In eukaryotes, a polypeptide can begin to fold to form a protein domain as soon as it emerges from the ribosomes, a process called co-translational folding. However, a domain can only completely fold when its entire sequence has emerged from the ribosome (Agashe et al., 2004; Netzer and Hartl, 1997). This scenario exposes non-native surfaces on nascent chains for a considerable length of time to interact non-specifically and make them highly aggregation prone.

In addition to the exposure of hydrophobic surfaces on nascent chains during cotranslational folding, the close proximity of nascent chains on polyribosomes may substantially increase their tendency to form non-native structures. However, cells have evolved systems which protect the emerging polypeptides from non-specific intra- or inter-molecular interactions. The first level of quality control is ensured by the arrangement of the polyribosomes on the mRNA. In both prokaryotes and eukaryotes, the polyribosomes are spatially positioned in staggered or pseudo-helical arrangement with their exit tunnels facing outwards. This arrangement maximizes the distance between emerging nascent polypeptide chains on adjacent ribosomes and hence serves to minimize the intermolecular interactions that otherwise may lead to aggregation and limit productive folding (Brandt et al., 2010; Brandt et al., 2009). As the environment in cells is highly crowded with various macromolecules and therefore not an ideal habitat for productive protein folding, an additional quality control mechanism at the level of chaperones becomes essential (Georgopoulos, 1992).

Cells encode a group of proteins called chaperones to prevent nascent chains from aberrant interactions. The term molecular chaperone was first used by Laskey and colleagues to describe a role of nucleoplasmin, an acidic nuclear protein that helps in the assembly of nucleosomes by neutralizing the non-specific ionic interactions between histones and DNA (Laskey et al., 1978). Later, John Ellis described chaperones as a ubiquitous family of proteins which mediate the correct folding of other polypeptides without being the component of the final functional structure (Ellis, 1987; Ellis, 1993).

Notably, chaperones by themselves do not provide any steric information for either polypeptide folding or in the oligomerization process; rather they use ingenious mechanism to promote the folding of nascent and newly synthesized polypeptide chains along the folding pathway. Chaperones, transiently and non-covalently, shield the exposed hydrophobic surfaces 
on their substrate clients and prevent their non-specific interactions with other molecules. These proteins, in some cases, can also facilitate the post-translational assembly of polypeptides into oligomeric structures as in the assembly of the functional enzyme Ribulose-1,5-bisphosphate carboxylase oxygenase (Rubisco) (Hemmingsen et al., 1988; Liu et al., 2010; Saschenbrecker et al., 2007). Many of the chaperones promote de novo folding by iterative cycles of substrate binding and release driven by ATP hydrolysis and may require co-factors or co-chaperones. Another common determinant evolutionary conserved in almost all the chaperone families is their specificity to recognize hydrophobic segments flanked by basic amino acid residues in their client proteins (Rousseau et al., 2006). This feature allows them to selectively recognize unfolded or misfolded proteins because they expose otherwise buried hydrophobic amino acid residues.

Although in cells there is a constitutive expression of all major chaperone classes, there is a remarkable increase in their cellular concentrations during conditions of stress (Ananthan et al., 1986; Bienz and Pelham, 1987). Such stress-induced chaperones are also called $\underline{H}$ eat $\underline{S}$ hock Proteins (HSPs) and they are generally classified according to their molecular weight, such as Hsp100, Hsp70, Hsp90, Hsp60, Hsp40 and small HSPs like Hsp27.

After more than three decades of research it is now well established that molecular chaperones are multifaceted and highly versatile molecules that are involved in diverse cellular functions, including de novo folding, oligomeric assembly, intracellular protein trafficking, refolding of stress denatured proteins and assistance in proteolytic degradation (Hartl and HayerHartl, 2009). A detailed discussion on their synthesis and their role in preventing protein aggregation during stress follows.

\section{II.4.1.1. Different Classes of Chaperones}

Chaperones are highly conserved in all three domains of life from archaea to bacteria to eukaryotic cells and they follow the same functional principles (Figure 3). They form a cellular folding network that helps to maintain the integrity of the cellular proteome both during normal growth and under stress conditions. Chaperone mediated folding is highly processive and it is coupled to translation and translocation which prevents the newly synthesized polypeptides from drifting into the bulk cytosol (Langer et al., 1992; Thulasiraman et al., 1999). Subsequently, 
chaperones are classified broadly into three main classes depending on their functional role at different stages of protein maturation.

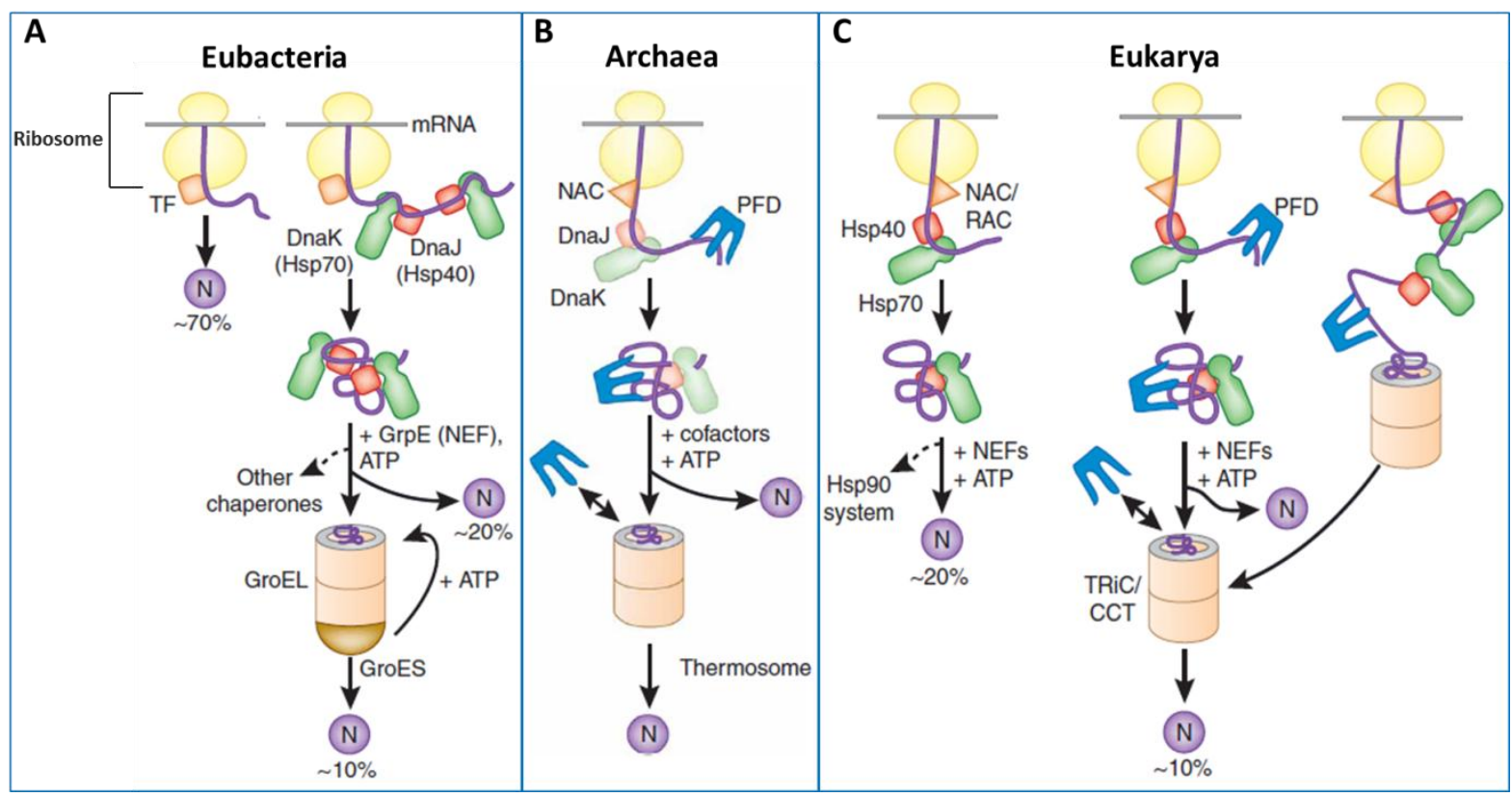

Figure 3: Organization of chaperone pathways in the three domains of life.

(a) In eubacteria, the nascent chains emerging from ribosomes interact with Trigger Factor (TF) and most of them ( 70\% of total) may fold rapidly into proteins upon release from the ribosomes without further assistance. Longer chains interact subsequently with DnaK (Hsp70) and DnaJ, chaperones which help in folding through several ATP-dependent iterative cycles of binding and release ( $20 \%$ of total). About $10 \%$ of total chains transit the GroEL and GroES chaperonin system to reach their native state (N). (b) In archaea, only some species contain DnaK/DnaJ. (c) In eukaryotes, similar to TF, nascent chain-associated complex (NAC) probably interacts with nascent chains. About $20 \%$ of chains reach their native states through assistance by ribosome associated chaperones (RAC), Hsp70 and Hsp40. A subset of chains requires Hsp90 for their folding. About $10 \%$ of chains are co- or post-translationally transferred to the chaperonin TRiC/CCT by Hsp70 and prefoldin (PFD). Adapted from (Hartl and Hayer-Hartl, 2009).

\section{II.4.1.1.1. Ribosome Associated Chaperones}

This category of chaperones includes proteins like Trigger factor (TF) (in bacteria),

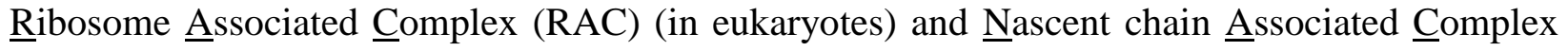
(NAC) (in both archaea and eukaryotes) that interact with newly synthesized nascent chains at the ribosome exit tunnel (Figure 3). Therefore, these ribosome associated chaperones are optimally positioned to interact with the majority of nascent chains destined to the cytosol. They all transiently bind to the exposed hydrophobic residues on polypeptide chains and prevent them from making non-specific intra or intermolecular contacts, thus delaying their premature co- 
translational folding (Agashe et al., 2004; Kaiser et al., 2006; Kramer et al., 2009). This provides sufficient time to the elongating polypeptide to receive its entire structural information requisite for the productive folding to begin.

Much of our current understanding about the functions and mechanism of this class of chaperones is based on the studies done with TF. TF is a $48 \mathrm{kDa}$ protein that binds to ribosomes in a 1:1 stoichiometry and interacts with nascent chains in an ATP independent manner (Hesterkamp et al., 1996). Recognition of ribosome bound polypeptide by TF is mediated by short sequences enriched in hydrophobic (particularly aromatic) residues (Patzelt et al., 2001). However, hydrophobicity alone is not the only determinant of affinity of TF for nascent chains because it has been shown that TF does not bind to the hydrophobic signal anchor sequence of FtsQ protein. TF can also recognize hydrophilic surfaces on certain folded domains of ribosomal protein S7 (Lakshmipathy et al., 2010). A recent study on the functions of TF suggests that bacterial outer membrane proteins are the most prominent substrates of TF and loss of TF results in premature, co-translational protein translocation (Oh et al., 2011). On the basis of these findings, similar functions of NAC and RAC can be envisioned. For instance, mutations in NAC have been shown to cause early embryonic lethality in higher eukaryotes, such as M. musculus, D. melanogaster and C. elegans (Bloss et al., 2003; Deng and Behringer, 1995; Markesich et al., 2000). However, direct evidence of these archaeal and eukaryotic ribosome bound chaperones in protein folding remains elusive.

\section{II.4.1.1.2. Non-ribosome Associated Chaperones}

The members of this class of chaperones are ubiquitously present in the cytosol of eubacteria, eukaryotes and some archaea, as well as in subcellular organelles such as the endoplasmic reticulum and mitochondria. They include members of the Hsp70 (DnaK) chaperone system and members belonging to the Hsp90 chaperone family that mediate the folding of longer nascent chains that failed to achieve their native state by ribosome associated chaperones (Figure 3). Since these chaperones participate in the folding process downstream, they do not bind directly to the ribosomes and are involved in both co- or post-translational folding of nascent chains. Chaperones belonging to this class show functional redundancy with ribosome associated chaperones whereby they hold and stabilize the nascent chains in a state competent for subsequent folding or translocation. The structure and mechanistic insight into the 
functions of the Hsp70 chaperone system and Hsp90 is presented in detail in the following subsections.

In eukaryotes and archaea, another protein complex called prefoldin or Gim complex (GimC) acts in a manner similar to Hsp70 to stabilize nascent chains. It is a $90 \mathrm{kDa}$ heterooligomeric complex involved in folding of actin and tubulin in Saccharomyces cerevisiae (Geissler et al., 1998; Hansen et al., 1999). Besides functioning in protein folding, prefoldin is also involved in channeling the substrates to the further downstream chaperones called chaperonins (Siegert et al., 2000; Vainberg et al., 1998).

\section{II.4.1.1.2.1. The Hsp70 Chaperone System}

The members of the Hsp70 family are highly conserved and ubiquitously distributed proteins found in all organisms from prokaryotes (DnaK) to eukaryotes (Hsp70). In eukaryotes, they are also present in subcellular organelles, mitochondria (e.g. Grp75) and endoplasmic reticulum (e.g. Grp78 or BiP or Kar2). There are multiple functionally redundant homologs of Hsp70 in the eukaryotic cytosol, such as in S. cerevisiae that contains four non-ribosome associated Hsp70s, Ssa1-4 and three ribosome-associated Hsp70s, called Ssb1, Ssb2 and Ssz1 (Lindquist and Craig, 1988; Nelson et al., 1992; Pfund et al., 1998). The cytosol of higher eukaryotes contains a constitutively expressed Hsp70 homolog called Hsc70 (ㅌeat $\underline{\text { Shock }}$ Cognate 70) and a stress inducible form, Hsp70. Henceforth, "Hsp70" will be generally used for different organisms and different compartments.

The crystal structure of Hsp70 shows that it consists of a $~ 44 \mathrm{kDa} \mathrm{N}$-terminal domain (ATPase domain) that mediates ATP binding and hydrolysis (Flaherty et al., 1990) and a 27 kDa C-terminal peptide binding domain (Bukau and Horwich, 1998; Zhu et al., 1996) (Figure 4a). The $\mathrm{C}$-terminal domain contains a $\beta$-sandwich subdomain with a peptide binding cleft and a $\alpha$-helical latch-like segment that acts as a lid to permit the entry and release of the substrate. Using a number of different approaches including phage display and synthetic peptide libraries, a consensus motif recognized by Hsp70 in substrate polypeptides has been identified. It has been found that Hsp70 has high affinity for short extended peptide stretches (approx. seven residues) enriched in aliphatic, hydrophobic residues (particularly leucine and isoleucine) in the central region flanked by positively charged residues (Blond-Elguindi et al., 1993; Flynn et al., 1991; Richarme and Kohiyama, 1993; Rudiger et al., 1997). Such binding sites for Hsp70 occur on 
average every $\sim 40$ residues in proteins and may account for the activity of Hsp70 to prevent aggregation (Rudiger et al., 1997). This substrate specificity allows Hsp70 to recognize structural features that are fingerprints of most nascent chains and enables it to shield unfolded polypeptides during translation and translocation and prevent them from premature folding. This binding affinity for hydrophobic side chains also allows Hsp70 to recognize misfolded and nonnative protein conformations with exposed hydrophobic residues. Quantitative immunoprecipitation analysis in mammalian cells demonstrated that Hsc70 transiently and preferentially associates with elongating polypeptides larger than $20 \mathrm{kDa}$ and at least $15-20 \%$ of newly synthesized proteins associate with Hsc70 during their biogenesis (Thulasiraman et al., 1999). These results are consistent with findings in prokaryotes where DnaK was shown to interact with $\sim 15 \%$ of polypeptides and has been shown to facilitate the post-translational folding of multi-domain proteins through several cycles of binding and release (Calloni, 2012; Teter et al., 1999).

Hsp70, like all other chaperones, transiently associates with its substrates and uses an ATP dependent mechanism to facilitate protein folding. The mechanistic elucidation of the reaction cycle of Hsp70 came from both in vitro and in vivo studies, primarily using DnaK which has served as a paradigm for all canonical Hsp70s (Figure 4b). Hsp70 has been shown to bind only to unfolded, but not to folded or native proteins in a temperature-dependent manner and the complex of Hsp70 with the nucleotide (ATP or ADP) modulates its intrinsic affinity for the polypeptide (Palleros et al., 1991; Pellecchia et al., 2000). In the ATP bound state, Hsp70 rapidly binds to its polypeptide in an open state where the latch over the peptide binding cleft is open. The hydrolysis of bound ATP by the N-terminal ATPase domain causes a conformational change leading to the closure of the latch and holding of the substrate molecule in a folding competent state (Liberek et al., 1991b; Palleros et al., 1994). 

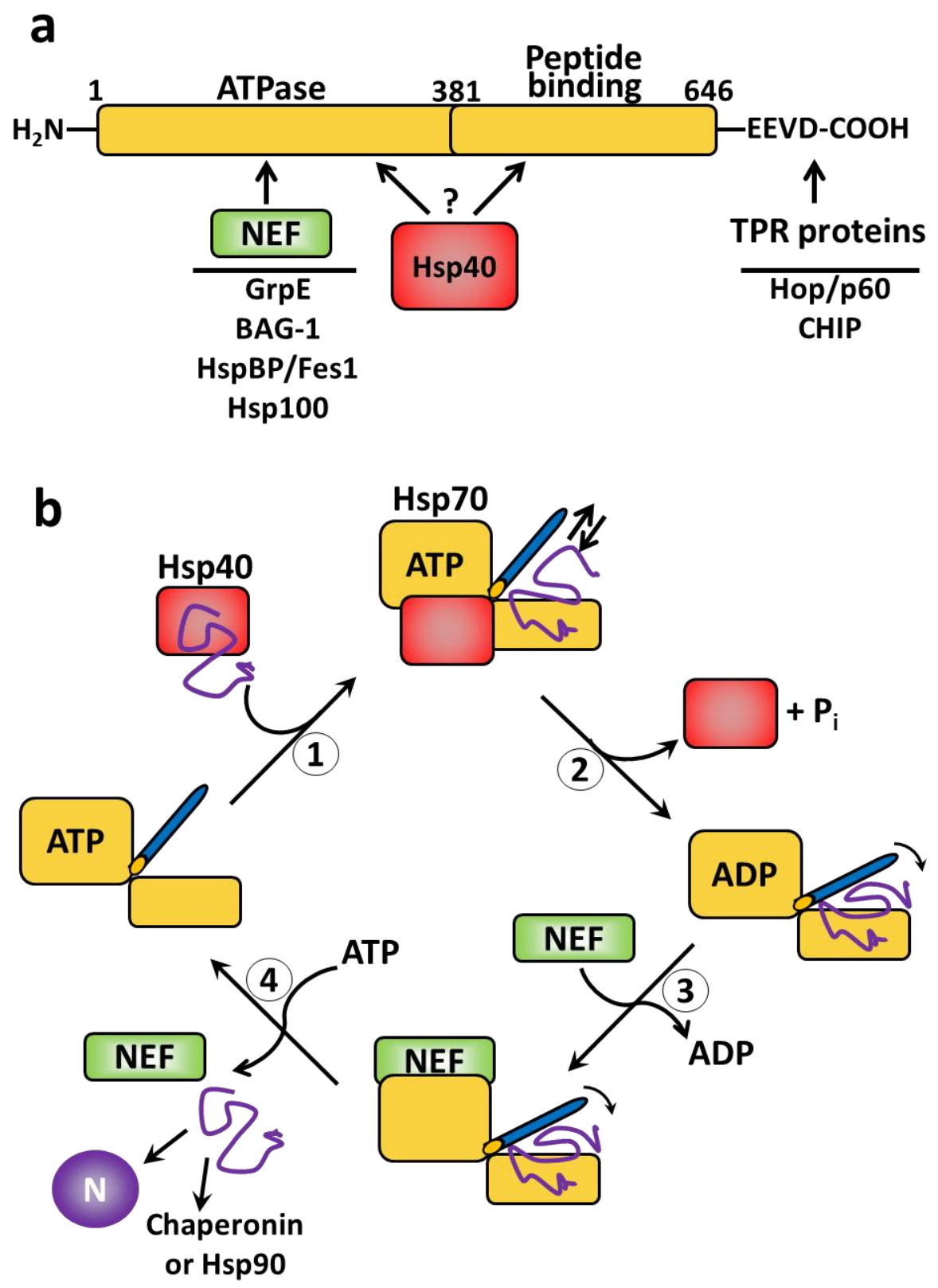

Figure 4: Structure and reaction cycle of the Hsp70 chaperone system.

(a) Domain architecture of Hsp70 showing the N-terminal ATPase domain and the C-terminal peptide binding domain (residue numbers refer to human Hsp70). The interaction of prokaryotic and eukaryotic cofactors with Hsp70 is shown schematically. Only the Hsp70 proteins of the eukaryotic cytosol contain an EEVD sequence at their C-terminus that mediates their interaction with tetratricopeptide repeat (TPR) containing proteins like HOP and CHIP. (b) Reaction cycle of Hsp70. (1) The reaction cycle starts with the Hsp40 (red) mediated delivery of unfolded substrate to ATP-bound Hsp70 (yellow). (2) Hsp40 stimulates ATPase activity of Hsp70 resulting in ATP hydrolysis and closure of the $\alpha$-helical lid (blue) to stabilize the Hsp70-substrate complex. Hsp40 dissociates from Hsp70 (3) Nucleotide exchange factor (NEF) (green) catalyzes the dissociation of ADP. (4) ATP binding to Hsp70 induces a conformational change leading to opening of $\alpha$-helical lid and release of substrate. (5) Released substrate either folds to a native state $(\mathrm{N})$, is transferred to downstream chaperones or rebinds to Hsp70 for another round of folding cycle. Adapted from (Hartl and Hayer-Hartl, 2009). 
The cycling of Hsp70 between its different nucleotide bound states is regulated by its two co-chaperones, Hsp40 and NEF (Nucleotide Exchange Factor) (Harrison et al., 1997; Liberek et al., 1991a; Mayer et al., 2000). Hsp40 (DnaJ) is a $41 \mathrm{kDa}$ J-domain containing protein whose N-terminal domain binds to Hsp70 and stimulates its ATPase activity. This results in ATP hydrolysis and concomitant stabilization of the Hsp70-substrate complex (Cyr et al., 1992; Mayer, 2010). The carboxyl terminal domain of Hsp40 has a substrate binding property and it can recognize aromatic and large aliphatic residues that allow it to recruit Hsp70 to target polypeptides in spatial proximity (Misselwitz et al., 1998; Rudiger et al., 2001; Szabo et al., 1996). Thus, Hsp40s provide substrate specificity to their partner Hsp70 molecules. Consequently, cells possess a variety of different Hsp40 molecules with different substrate binding properties which further broadens the activity spectrum of Hsp70 (Kampinga and Craig, 2010). In addition to Hsp40, NEFs play a pivotal role in the functional cycle of Hsp70. These protein molecules bind to the ATPase domain of Hsp70 and by modifying the conformation of the nucleotide binding pocket, promote the release of bound ADP (Figure 4). This allows the rebinding of another ATP molecule, which then triggers the release of substrate from the peptide binding cleft and thus completes the Hsp70 folding cycle (Harrison et al., 1997; Szabo et al., 1994). In prokaryotes, GrpE (23 kDa) acts as a NEF for DnaK and it is also present in eukaryotes in organelles of symbiotic origin (i.e. mitochondria and chloroplasts). Eukaryotic cytosol contains the BAG-1 protein and proteins belonging to the Hsp110 family that act as a NEF for Hsp70 (Dragovic et al., 2006; Hohfeld and Jentsch, 1997; Polier et al., 2008; Raviol et al., 2006).

Since members of the Hsp70 family, together with their co-chaperones, function at a very crucial junction in the hierarchical structure of protein folding helpers, they are functionally integrated with other chaperone members and they co-ordinate with each other to provide a protective folding compartment (Langer et al., 1992; Thulasiraman et al., 1999). For instance, it was demonstrated in E. coli that deletion of DnaK in TF-deleted cells resulted in massive aggregation of cytosolic proteins and combined deletion of both TF and DnaK caused synthetic lethality (Calloni, 2012; Deuerling et al., 1999). Further, upon deletion of TF, the polypeptide flux through DnaK increases from $\sim 15 \%$ to $\sim 40 \%$ showing that there is partial functional redundancy between different classes of chaperones (Teter et al., 1999). Using firefly luciferase as a model protein, it was shown in rabbit reticulocyte lysate (RRL) that Hsc70 together with Hsp40 and TRiC sequentially mediate the folding of luciferase nascent chains and depletion of 
either of these chaperones disrupts the highly organized chaperone pathway (Frydman et al., 1994). In S. cerevisiae, it was shown that Hsp70 and TRiC cooperate in the folding and assembly of the on Hippel-Lindau (VHL) tumor suppressor complex (Melville et al., 2003). Thus, Hsp70 synergizes its activity with other chaperones and co-chaperones to effectively fold cellular proteins and lack of one chaperone component generally causes other chaperone members to take over its functions.

Hsp70 members are highly multifunctional proteins that have been shown to play a key role in proteome maintenance, such as in de novo protein folding (co- or post-translational), protein translocation across membranes (Lyman and Schekman, 1997; Matlack et al., 1999; Young et al., 2003), refolding of stress damaged proteins (Ben-Zvi et al., 2004; Goloubinoff et al., 1999; Schroder et al., 1993; Sharma et al., 2010), in preventing protein aggregation (Auluck et al., 2002; Broadley and Hartl, 2009; Klucken et al., 2004; Sakahira et al., 2002; Warrick et al., 1999), disaggregation (Ben-Zvi and Goloubinoff, 2001; Diamant et al., 2000; Liberek et al., 2008; Shorter, 2011) and degradation of irreparable misfolded proteins (Bercovich et al., 1997; Fisher et al., 1997; Urushitani et al., 2004). These essential and diverse cellular functions of Hsp70 are attributed to its physical interaction with various co-chaperones such as Hsp40, NEFs and with proteins such as HIP, HOP and CHIP.

HIP, Hsp70 Interacting Protein, is a $\sim 48 \mathrm{kDa}$ eukaryotic protein that was identified in a yeast two-hybrid screen (Hohfeld et al., 1995). HIP has been shown to interact with the ATPase domain of Hsp70 by its tetratricopeptide repeat (TPR) region (Velten et al., 2000) and this interaction slows dissociation of ADP from Hsp70. This stimulates the chaperone activity of Hsp70, presumably because it stabilizes the Hsp70 substrate complex by preventing premature substrate release (Hohfeld et al., 1995). In this regard, HIP is antagonist to BAG-1 which promotes the release of the bound ADP from Hsp70 and results in substrate release (Hohfeld and Jentsch, 1997; Takayama et al., 1997). Recently, HIP together with Hsc70 has been shown to significantly reduce inclusion formation in an in vitro model of Spinal Bulbar Muscular Atrophy (SBMA) and a primary neuronal model of polyglutamine disease (Howarth et al., 2009).

HOP, $\underline{H}$ sp70-ㅍsp90 Organizing Protein, a $60 \mathrm{kDa}$ protein (also called Sti1 or p60 in yeast) was first identified in a genetic screen to play a role in heat shock response of some Hsp70 genes (Nicolet and Craig, 1989; Smith et al., 1993). Subsequently, it was shown that HOP is a 
functional homolog of the BAG-1 protein that stimulates nucleotide exchange by Hsp70 (Gross and Hessefort, 1996). HOP interacts with Hsp70 and Hsp90 via its three TPR domains and plays an essential role in coupling Hsp70 to Hsp90 (Scheufler et al., 2000; Schmid et al., 2012). For example, it was shown in S. cerevisiae that association of HOP with Hsp70 and Hsp90 redirects VHL tumor suppressor protein from the folding pathway to the ubiquitin-proteasome mediated degradation pathway (McClellan et al., 2005). HOP has also been shown to stimulate refolding of thermally denatured firefly luciferase by bridging the interaction between Hsp70 and Hsp90 (Johnson et al., 1998).

CHIP, Carboxyl-terminus of $\underline{\text { Hsc70 }}$ Interacting Protein is a cytoplasmic $35 \mathrm{kDa}$ protein which possesses three N-terminal TPR domains that bind to the C-terminus of Hsp70 and Hsp90 molecular chaperones (Ballinger et al., 1999). CHIP has been shown to inhibit the Hsp40 induced stimulation of ATPase activity of Hsp70 and, thus, acts as a negative regulator of the Hsp70 reaction cycle (Ballinger et al., 1999; Stankiewicz et al., 2010). The C-terminus of CHIP possesses an E3 ubiquitin ligase like activity that allows it to ubiquitylate irreparable Hsp70 and Hsp90 bona fide substrates (in vitro and in vivo) and targets them for the ubiquitin-proteasome mediated degradation pathway (Connell et al., 2001; Murata et al., 2001). CHIP together with Hsc70 has been shown to ubiquitylate immature forms of cystic fibrosis transmembrane conductance regulator (CFTR) protein and mutant superoxide dismutase 1 (SOD1) protein and facilitate their degradation (Meacham et al., 2001; Urushitani et al., 2004). Both CFTR and SOD1 are implicated in protein misfolding diseases, cystic fibrosis disease and familial amyotrophic lateral sclerosis (ALS) disease, respectively. A recent study suggests that CHIP has an intrinsic chaperone like activity that enables it to selectively recognize and bind misfolded proteins. This function of CHIP is temperature sensitive which may allow CHIP to target heat denatured proteins directly for degradation (Rosser et al., 2007). Therefore, CHIP plays a pivotal role in cellular triage decisions that regulate the balance between folding and degradation of chaperone substrates.

\section{II.4.1.1.2.2. The Hsp90 Chaperone System}

The dimeric heat shock protein 90 (Hsp90) is a highly conserved protein found in all organisms from bacteria to mammals except archaea. Using genomics and bioinformatics approaches, a study has shown that Hsp90 family members underwent multiple duplications and 
also subsequent losses during their evolution. This study has classified the members of the Hsp90 family into 5 subfamilies: (i) cytosolic Hsp90A, (ii) endoplasmic reticulum (ER)localized Hsp90B, (iii) chloroplast Hsp90C, (iv) mitochondrial TNFR-associated protein (TRAP) and (v) bacterial High temperature protein $\underline{G}(\mathrm{HtpG})$ (Chen et al., 2006). The cytosolic Hsp90A is further divided into Hsp90AA (constitutively expressed isoform) and Hsp90AB (inducible isoform during stress).

While the eubacterial HtpG is dispensable under normal growth conditions (Bardwell and Craig, 1988), the Hsp90 in eukaryotes is one of the most abundant ( 1-2\% of total cytosolic proteins) and essential protein for cell viability (Borkovich et al., 1989). This means in eukaryotes Hsp90 could have many diverse cellular functions other than chaperoning folding intermediates. Indeed, Hsp90 was first identified during affinity purification of the protooncogenic tyrosine kinase v-Src protein where it co-immunoprecipitated with v-Src from (Rous sarcoma) virus-transformed chicken cells (Brugge et al., 1981). Later, Hsp90 was found to be associated with several different clients such as protein kinases and nuclear steroid receptors (e.g. glucocorticoid receptor) (Joab et al., 1984; Schuh et al., 1985; Smith, 1993; Smith et al., 1992). Since then, Hsp90 clients have grown tremendously owing to genome-wide high throughput studies. For instance, in S. cerevisiae, 1,022 unique proteins interact genetically and 505 proteins interact physically with Hsp90, making Hsp90 one of the most highly connected proteins in the yeast genome network (http://thebiogrid.org/35923/summary/saccharomycescerevisiae/hsp82.html). Although protein kinases and steroid hormone receptors are the best understood clients of Hsp90, other essential proteins such as transcription factors, chromatin remodeling factors and proteins involved in cell signaling unequivocally require Hsp90 for their structural maturation and functional regulation (Sharma et al., 2012; Taipale et al., 2010). Because of its central position in numerous vital regulatory hubs, Hsp90 is absolutely essential for stable propagation of cancerous cells (Kubota et al., 2010; Neckers, 2007; Pick et al., 2007; Whitesell and Lindquist, 2005). 




Figure 5: Structure and ATPase cycle of the Hsp90 chaperone system.

(a) Domain structure of Hsp90 consisting of amino-terminal domain (NTD), middle domain (MD) and carboxy-terminal domain (CTD). (b) Schematic of conformational cycle of Hsp90. In ADP-bound state, Hsp90 is present in open or relaxed state. ATP binding to the ATPase domain of NTD induces a conformational rearrangement in Hsp90 and the subsequent closure of the ATP lid in the NTD. After lid closure, the NTDs dimerize, forming the closed Hsp90 dimer. This ATP-bound state of Hsp90 is referred to as closed or tense state and this state is committed for ATP hydrolysis. After ATP hydrolysis, the NTDs dissociate and both monomers separate amino-terminally. The inactive substrate molecule interacts mostly with the middle domain (MD) and is conformationally activated as Hsp90 proceeds through the ATPase cycle. Adapted from (Taipale et al., 2010).

Hsp90 exits as a dimer in the functional active state. As shown in Figure 5a, the monomer contains a highly conserved amino-terminal domain (NTD) connected to a middle domain via a charged linker. The NTD has a nucleotide binding pocket and has a weak intrinsic ATPase activity (Obermann et al., 1998; Prodromou et al., 1997). Several conserved residues in the NTD form a molecular lid that closes over the nucleotide binding pocket in its ATP bound state (closed or tense state). In the closed state, a slow transient interaction of the NTDs of each monomer facilitates ATPase activity (Cunningham et al., 2008; Prodromou et al., 2000). 
Hydrolysis of ATP with subsequent dissociation of ADP restores Hsp90 to its original open or relaxed state (Sullivan et al., 1997).

The ATPase cycle has been shown to result in large and highly dynamic conformational rearrangements that enable Hsp90 to recognize its diverse client repertoire and are essential for client maturation (Csermely and Kahn, 1991; Hessling et al., 2009) (Figure 5b). Some naturally occurring small molecule compounds like geldanamycin bind to the ATP binding pocket of Hsp90 with high affinity and specificity. This causes the displacement of ATP and functional arrest of the Hsp90 chaperone cycle (Stebbins et al., 1997; Whitesell and Lindquist, 2005). The middle domain is followed by a carboxy-terminal domain (CTD) which mediates dimerization and is less conserved in sequence (Harris et al., 2004; Minami et al., 1994). The five C-terminal residues (MEEVD motif) form a highly conserved TPR domain binding site that allows Hsp90 to interact with a number of co-chaperones containing TPR domains (Pearl and Prodromou, 2006; Young et al., 1998).

Like the Hsp70 family members, Hsp90 function is also modulated by its sequential cooperation with different co-chaperones that regulate the ATPase activity of Hsp90 and hence its interaction with the diverse substrates ( $\mathrm{Li}$ et al., 2012; Prodromou et al., 1999). Cochaperones such as HOP and p23 inhibit Hsp90's ATPase activity and are likely to be involved in client loading or the formation of a Hsp90-client substrate complex (McLaughlin et al., 2006; Schmid et al., 2012; Southworth and Agard, 2011; Young and Hartl, 2000). The co-chaperone AHA1 (Activator of Hsp90 ATPase homologue 1) stimulates the Hsp90 conformational cycle by enhancing the ATPase activity and permitting the substrate release for the next maturation step (Meyer et al., 2004; Panaretou et al., 2002). Some co-chaperones like HOP and CHIP play an essential role in facilitating the cooperative and successive action of Hsp40, Hsp70 and Hsp90 on client proteins to promote either folding or degradation. For example, in the maturation of progesterone receptor (PR), Hsp40 first docks onto the unfolded PR polypeptide and then recruits the Hsp70-ATP complex. Hydrolysis of ATP triggers the formation of the stable complex Hsp70-ADP-PR. Hsp90-HOP then binds Hsp70-ADP, allowing transfer of PR to Hsp90. The final maturation step of PR involves the binding of p23 and ATP to Hsp90, which then leads to the dissociation of HOP and Hsp70 (Cintron and Toft, 2006). Repeated failure of the maturation step of the client by Hsp90 results in the degradation of the substrate by CHIP. 
CHIP has also been reported to remodel the Hsp90-client complex by impairing the binding of p23 which is required for the late maturation steps of Hsp90 clients and promotes their degradation through the proteasome (Connell et al., 2001). Thus, a fine balance between protein folding and degradation mediated by Hsp90 critically depends on the cellular concentrations and affinities of the co-chaperones (Kundrat and Regan, 2010).

Apart from its physiological function, Hsp90 plays a critical role during stress conditions. Early evidence for the role of Hsp90 in protein folding came from in vitro studies where it was demonstrated that chemically denatured substrates like citrate synthase (mainly $\alpha$-helical structure) and Fab fragment of a monoclonal antibody (only $\beta$-sheets present) can be efficiently refolded with a high yield in the presence of purified Hsp90 (Wiech et al., 1992). It was also shown in vitro that Hsp90 primarily functions in preventing aggregation of stress-denatured proteins and maintains them in a folding competent state (Freeman and Morimoto, 1996; Yonehara et al., 1996). Further, in vivo studies using firefly luciferase as a model substrate have documented the role of $\mathrm{Hsp} 90$ in protecting the proteins during stress and its role in refolding of non-native structures and degradation of terminally misfolded proteins during recovery from stress (Schneider et al., 1996). Hsp90-HOP, together with Hsp70-Hsp40 functions as a folding machine, also called foldosome that participates in the refolding of stress-denatured proteins during the recovery phase and prevents aggregation of misfolded proteins (Hutchison et al., 1994; McClellan et al., 2007; Powers et al., 2008; Schumacher et al., 1996; Walerych et al., 2009; Wegele et al., 2006). The Hsp90 reservoir, besides buffering proteostasis against environmental stress, is also involved in safeguarding protein functions in context of genetic variation. Hsp90 maintains mutant proteins in their functional state and hence silences the preexisting mutations in the genetic background. However, during stress, the Hsp90 reservoir is depleted which allows the emergence of hidden phenotypes from the mutant proteins that can be tolerated by cells, leading to genetic evolution (Jarosz and Lindquist, 2010; Queitsch et al., 2002; Rutherford and Lindquist, 1998).

\section{II.4.1.1.3. The Chaperonins}

Further downstream of non-ribosome associated chaperones, at the distal end of the folding pathway, large cylindrical protein machines called chaperonins participate in the folding of newly synthesized polypeptides (Figure 3). The chaperonins are double-ring complexes of 
$\sim 800 \mathrm{kDa}$ conserved in all domains of life. They are nano-cage compartments enclosing a central cavity where proteins in non-native states can be encapsulated for folding process (Viitanen et al., 1992). Unlike ribosome bound chaperones (TF, NAC and RAC) and Hsp70, that primarily functions in the de novo folding process by holding newly synthesized chains in a folding competent state, the cylindrical chaperonin complexes are presumed to work like an Anfinsen's cage where the unfolded polypeptide in the central cavity of chaperonin is secluded from the cellular milieu. Under these conditions, similar to infinite dilution, the unfolded polypeptide achieves its native state according to its thermodynamic potential (Ellis, 1994). However, experimental evidence suggests that like all other ATP dependent chaperones, the chaperonins fold the polypeptides via iterative annealing mechanism of substrate binding and release at the expense of energy from ATP hydrolysis. For example, the GroEL-GroES system has been shown to actively participate in the folding process by causing steric confinement of the protein. This reduces the entropic folding barrier of the intermediate and strongly accelerates native state formation (Chakraborty et al., 2010).

Depending on the architecture and sequence similarity, the chaperonins have been divided into two distinct subgroups (Figure 6). Group I chaperonins, such as GroEL (L for large) in Escherichia coli and Hsp60 in organelles of symbiotic origin (mitochondria and chloroplasts), require a co-factor/co-chaperonin called GroES (S for small) or Hsp10 for their functions. GroES or Hsp10 acts like a lid that sits on the GroEL central cavity and helps in protein encapsulation. Group II chaperonins such as thermosome in archaea and TRiC in eukaryotes (TCP-1 Ring


encapsulate proteins in their cavity. They have a built-in lid formed by the protrusion of a $\alpha$ helical insertion in their apical domain (Ditzel et al., 1998; Klumpp et al., 1997).

The bacterial chaperonin GroEL and its co-chaperone GroES are indispensable for the viability of E. coli under all growth conditions (Fayet et al., 1989; Horwich et al., 1993). Structurally, GroEL consists of two heptameric rings of identical subunits of $57 \mathrm{kDa}$ each, stacked back-to-back (Braig et al., 1994). GroES, on the other hand, is a dome-shaped homoheptameric ring of $10 \mathrm{kDa}$ subunits each that binds to the ends of the GroEL cylinder in the presence of ATP (Hunt et al., 1996) (Figure 6a). The GroEL-GroES machine is a wellorchestrated system where binding of GroES to GroEL leads to allosteric modulation in the 
GroEL subunits (Xu et al., 1997). This results in expansion of the central cavity, enough to encapsulate a polypeptide of $\sim 60 \mathrm{kDa}$ (Hartl and Hayer-Hartl, 2002; Sakikawa et al., 1999; Tang et al., 2006). Generally, it takes $~ 10 \mathrm{sec}$ for the ATP hydrolysis which drives the folding of intermediates to native tertiary structure inside the GroEL cavity. However, if the substrate still exposes hydrophobic residues then it is immediately captured for the next round of the folding cycle (Corrales and Fersht, 1996; Mayhew et al., 1996). It has been shown in vitro that GroEL can bind about half of the total soluble proteins of E. coli in denatured state (Viitanen et al., 1992) and in vivo approximately 10-15\% of all cytoplasmic proteins (under normal growth) and upto 30\% of stress denatured proteins interact with GroEL (Ewalt et al., 1997; Houry et al., 1999; Kerner et al., 2005).
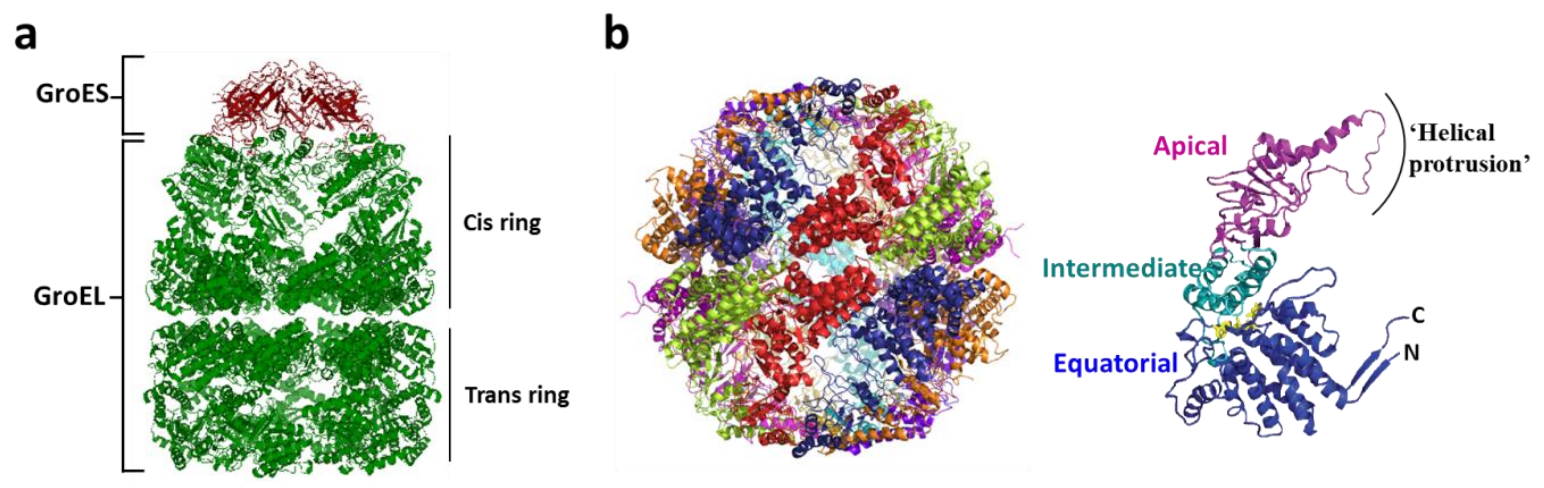

Figure 6: Structure of the chaperonin system in prokaryotes and eukaryotes.

(a) Crystal structure of the asymmetric GroEL-GroES complex in prokaryotes (Protein Data Bank: $1 \mathrm{AON}$ ), showing the GroES-bound chamber of GroEL (called cis ring) and the opposite GroEL ring (called trans ring). Adapted from (Xu et al., 1997). (b) left: Crystal structure of the eukaryotic chaperonin TRiC/CCT (Protein Data Bank: 3P9D), showing two heterogeneous 8-membered rings stacked back-toback. Right: domain structure of one of the eight TRiC subunits consisting of equatorial domain (blue), intermediate domain (cyan) and apical domain (magenta). The $\alpha$-helical protrusion in the apical domain acts as a built-in lid for substrate encapsulation. Adapted from (Dekker et al., 2011).

Group II chaperonins also function in a similar manner as the GroEL/ES system in ATP dependent substrate encapsulation in the chaperonin cavity (Meyer et al., 2003). Though they also have a double ring structure like group I chaperonins, they are more heterogeneous in sequence and structure. For example, thermosome has two or three different subunits per complex arranged in eight or nine fold symmetrical rings. TRiC from eukaryotes is a heterooligomeric, toroid shaped complex consisting of eight different subunits per ring and each subunit is $\sim 50-60 \mathrm{kDa}$ in size (Figure 6b). Interestingly, Group II chaperonins share sequence 
homology with GroEL at the ATP binding site, but they differ considerably in sequence of the substrate binding site (Kim et al., 1994; Spiess et al., 2006). TRiC, like GroEL, is an essential protein since at least two cytoskeletal proteins, actin and tubulin, are obligate substrates of TRiC (Dobrzynski et al., 1996; Gao et al., 1992; Llorca et al., 1999; Yaffe et al., 1992). Unlike GroEL/ES which can act only post-translationally, TRiC has been shown to fold the discrete domains of firefly luciferase co-translationally (Frydman et al., 1994). From biochemical studies using unfolded firefly luciferase, actin and tubulin, it has been shown that while GroEL/ES failed to fold these model proteins, TRiC was able to mediate their folding, suggesting that it can interact with a different range of substrates via mechanism distinct from class I chaperonins (Frydman et al., 1992; Tian et al., 1995; Yam et al., 2008). A recent study of the TRiC interactome suggests that $\sim 10 \%$ of newly synthesized cellular proteins, including actin, tubulin, cell cycle regulators and tumor suppressors are TRiC substrates. Generally, multidomain proteins ranging from 40-75 $\mathrm{kDa}$ are ideal substrates (Yam et al., 2008). This study also showed that proteins belonging to oligomeric assemblies are highly enriched in the interactome and this suggests a role of $\mathrm{TRiC}$ in facilitating protein complex assembly in cells.

\section{II.4.2. HSF1 and Stress Response}

Both prokaryotes and eukaryotes have an evolutionary conserved mechanism to effectively respond to changes inflicted by their environment (global or local). This mechanism was first observed as a temperature-induced puffing pattern in polytene chromosomes of Drosophila melanogaster larvae salivary glands (Ritossa, 1962). It is now broadly referred to as heat shock response. A decade later, many research studies showed that the heat shock response resulted in a robust activation of a subset of genes encoding the heat shock proteins (HSPs) that primarily restore the disturbed biochemical landscape of a cell (Lindquist, 1986; Morimoto, 1993).

Further insight into the mechanism of the heat shock response outlined the following highly conserved features that are either prerequisites or the consequences of cellular stress response (Figure 7 ).

1. The heat shock response is mediated at the transcription level by an array of highly conserved inverted repeats (nGAAn), called as $\underline{\text { Heat }}$ Shock Elements (HSEs) that are present in multiple copies, upstream of the hsp genes (Amin et al., 1988; Pelham, 1982). 
2. The transcriptional induction of stress response upon proteotoxic insults is led by a specific transcription regulator, $\underline{\text { Heat }} \underline{\text { Shock }}$ Factor (HSF) that selectively binds to the HSEs and induces transcription of HSPs (Abravaya et al., 1991; Kingston et al., 1987; Parker and Topol, 1984; Wu, 1984). Yeast, fruit fly, and nematode possess only a single HSF whereas mammals have three different HSFs, HSF1, HSF2 and HSF4 (Akerfelt et al., 2007). Distinct HSFs exhibit tissue-specific patterns of expression and contribute to diverse physiological and developmental processes. Among different HSFs, HSF1 is considered as a master regulator for induction of $h s p$ genes during stress (Morimoto, 1998; Trinklein et al., 2004).

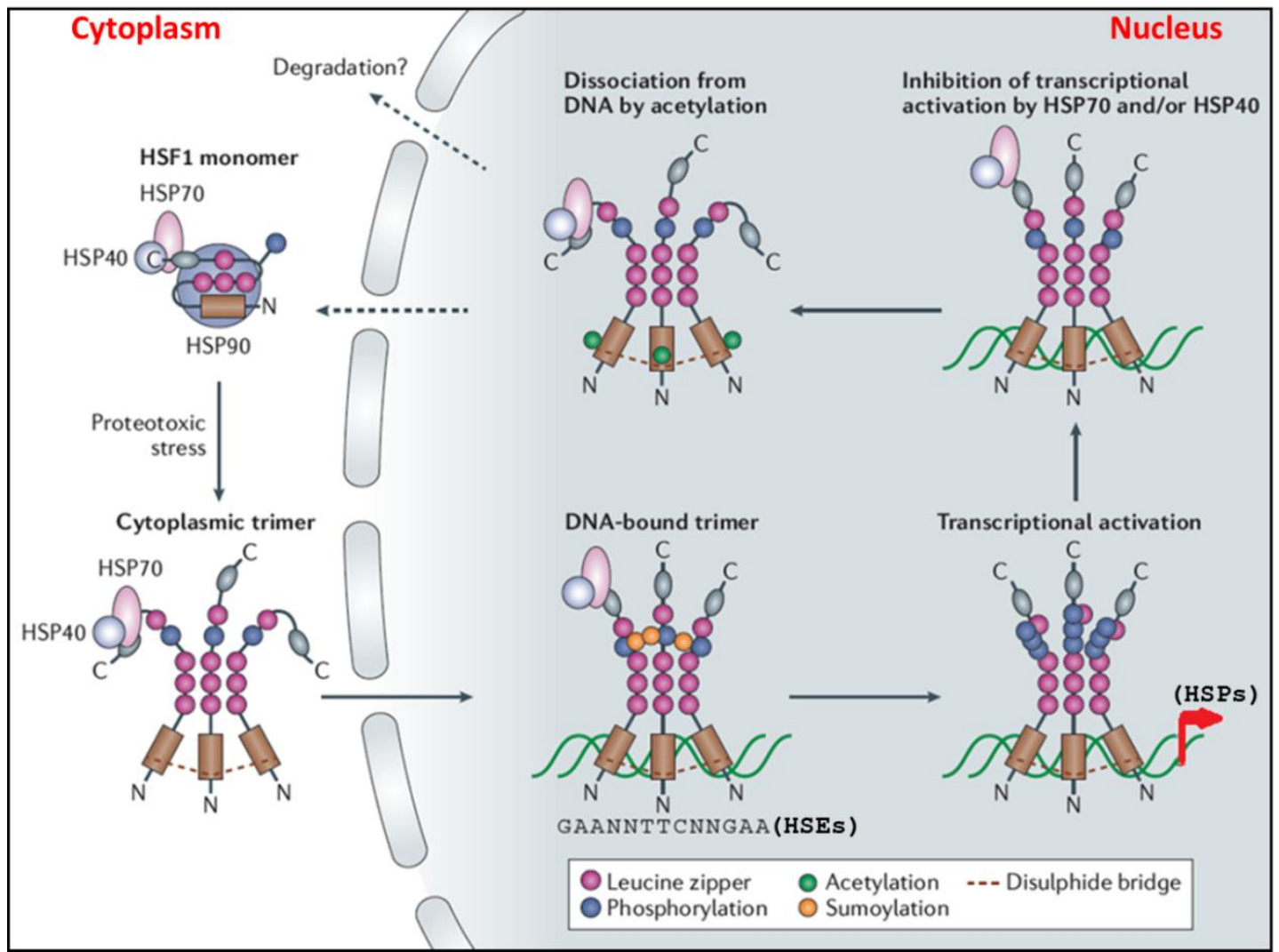

\section{Figure 7: HSF1 mediated heat shock response.}

Under normal conditions, Heat Shock Factor 1 (HSF1) exists as an inactive monomer in a complex with Hsp70, Hsp40 and Hsp90 in the cytoplasm. Stress conditions lead to protein unfolding and misfolding in the cytoplasm which results in dissociation of the chaperone complex from HSF1. HSF1 then trimerizes and translocates to the nucleus where it undergoes post-translational modifications including acetylation, sumoylation that render it transcriptionally active. Activated HSF1 trimer induces the transcription of $h s p$ genes, resulting in the translation of HSPs, including Hsp70 and Hsp90. The increased cellular concentration of these chaperones binds to HSF1 and attenuates its activity by negative feedback mechanism. Adapted from (Neef et al., 2011). 
3. Under normal physiological conditions, monomeric HSF1 is diffusely distributed in cytoplasm and nucleus and is kept in an inactive complex with Hsp70, Hsp40 and Hsp90 (Ali et al., 1998; Mosser et al., 1993; Nadeau et al., 1993; Zou et al., 1998). During proteotoxic stress, the structure and function of proteins is compromised, leading to the depletion of the chaperone reservoir. As a result, the chaperone complex from HSF1 dissociates and free HSF1 monomers assemble into a trimer (Sorger and Nelson, 1989). Extensive post-translational modifications such as acetylation, phosphorylation and SUMOylation renders HSF1 active (Akerfelt et al., 2010). The active HSF1 trimer then translocates to the nucleus and binds to the HSEs to induce transcription of HSPs (Baler et al., 1993; Perisic et al., 1989; Sarge et al., 1993; Westwood et al., 1991). Thus, the heat shock response is initiated by the presence of misfolded and damaged proteins (Ananthan et al., 1986; Baler et al., 1992). Some aggregation prone proteins like those involved in Huntington's disease (mutant huntingtin) and Parkinson's disease (mutant $\alpha$-synuclein) fail to properly induce the stress response (Hay et al., 2004; Prahlad and Morimoto, 2009; Zourlidou et al., 2007) which may contribute to the pathology of neurodegeneration.

4. The HSPs shield non-native or misfolded proteins and help in recovery from proteostasis imbalance. Once the chaperone reservoir reverts to normal, the HSF1 activation is attenuated by a negative feedback mechanism upon rebinding of the chaperone complex and post-translational modifications (Abravaya et al., 1992; Baler et al., 1992; Guo et al., 2001; Shi et al., 1998).

This multistep and highly regulated activation of the heat shock response culminates in restoration of proteostasis. However, failure in eliciting a stress response can lead to global instability of the proteome and may result in accumulation of misfolded proteins which can engage in non-specific interactions to form aggregates. Therefore, many studies are now focusing on increasing the stress response via HSF1 activation to provide a potential therapeutic edge to diseases of proteostasis deficiency (Neef et al., 2011).

\section{II.4.3. Protein Misfolding and Aggregation}

During its lifetime, an organism is under constant stress such as environmental (e.g. nutrient balance), physical (e.g. heat stress), chemical (e.g. oxidative stress) and 
pathophysiological (e.g. ischemia, bacterial infection etc.). Destabilizing mutations in the genetic background, translational errors and chronic stress like aging further risk the integrity of the proteome (Drummond and Wilke, 2008; Gidalevitz et al., 2010). Despite the activation of stress response and the existence of various protein surveillance mechanisms including molecular chaperones and degradation pathways, the load of non-native protein species can overwhelm the cellular quality control system during proteotoxic insults. Under these circumstances, proteins fail to fold correctly or to retain their biologically active state. This may result in exposure of otherwise buried hydrophobic residues and cause protein misfolding (Dobson, 1999, 2003). During extreme or prolonged stress, misfolded proteins significantly populate the crowded cellular milieu and engage in non-specific intra- or inter-molecular interactions which may result in the formation of aggregates or inclusion bodies (IBs). IBs can be cytoplasmic and/or nuclear and sometimes they are formed in the perinuclear area, close to the microtubule organizing centre (MTOC) in eukaryotic cells. These IBs around the MTOC, referred as aggresomes contain ubiquitinated misfolded proteins and are formed in a microtubule dependent, active process, caged by the intermediate filament protein vimentin (Johnston et al., 1998; Kopito, 2000).

If these products of off-pathway reaction are not cleared either by refolding, by resolubilizing or by degradation, their accumulation can lead to proteinopathies and cellular toxicity. Indeed, many neurodegenerative disorders such as Alzheimer's disease (AD), Parkinson's disease (PD), Huntington's disease (HD), amyotrophic lateral sclerosis (ALS) and prion-based diseases are protein conformational diseases associated with a gain-of-function phenotype (Table 1). In these diseases, the causative proteins form amyloid fibrils or plaques consisting of SDS insoluble and heat stable cross $\beta$-pleated sheets that run perpendicular to the long axis of the fibril (Chiti and Dobson, 2006; Sunde and Blake, 1997). These highly ordered amyloid fibrils can be cytoplasmic, nuclear or even extracellular and are generally formed via nucleation based linear pathways from monomers to oligomers to protofibrils to fibrils (Kiefhaber et al., 1991; Sipe, 1992). Despite the often ubiquitous expression of disease-related proteins, only a selective population of neurons gets affected in these neurodegenerative diseases. 
Table 1: Clinical and biochemical features of neurodegenerative diseases. Adapted from (Soto, 2003).

\begin{tabular}{|c|c|c|c|}
\hline Disease & Clinical features & Proteins involved & $\begin{array}{c}\text { Cellular location of } \\
\text { aggregates }\end{array}$ \\
\hline Alzheimer's (AD) & Progressive dementia & Amyloid- $\beta$ and tau & $\begin{array}{l}\text { Extracellular, } \\
\text { cytoplasmic }\end{array}$ \\
\hline Parkinson's (PD) & Movement disorder & $\alpha$-Synuclein & Cytoplasmic \\
\hline Huntington's (HD) & $\begin{array}{l}\text { Dementia, motor and } \\
\text { psychiatric problems }\end{array}$ & Huntingtin & Nuclear \\
\hline $\begin{array}{l}\text { Amyotrophic lateral } \\
\text { sclerosis (ALS) }\end{array}$ & Movement disorder & Superoxide dismutase & Cytoplasmic \\
\hline $\begin{array}{c}\text { Transmissible } \\
\text { spongiform } \\
\text { encephalopathies }\end{array}$ & $\begin{array}{l}\text { Dementia, ataxia, } \\
\text { psychiatric problems } \\
\text { and insomnia }\end{array}$ & Prion protein & Extracellular \\
\hline
\end{tabular}

All the protein conformational diseases listed above are associated with pleiotropic effects that contribute to the generic cellular toxicity by the respective causative proteins (Figure 8). Toxicity can be due to the exposure of normally buried moieties such as hydrophobic side chains or free main chain $\mathrm{NH}$ and $\mathrm{CO}$ groups that can lead to non-native hydrogen bond interactions with other proteins (Mossuto et al., 2010). Indeed, several studies have shown that sequestration of metastable and essential cellular factors such as proteins involved in chromatin remodeling, transcription, translation, nuclear import and cytoskeletal structure by the aggregates is the major cause of observed toxicity in vivo (Bucciantini et al., 2002; Chai et al., 2002; Olzscha et al., 2011; Suhr et al., 2001). Aggregates can also interfere with the cellular defense mechanisms by altering protein folding homeostasis (Gidalevitz et al., 2006; Satyal et al., 2000), by blocking proteasome mediated degradation (Bence et al., 2001; Bennett et al., 2005) or by inhibiting autophagy (Cuervo et al., 2004). Other models suggest that the aggregates can engage in aberrant interaction with cellular membranes leading to the formation of membrane pores (Lashuel et al., 2002) or they can disturb cellular ion homeostasis (Quist et al., 2005), may cause mitochondrial dysfunction and oxidative stress (Muller et al., 2010) (Figure 8). 


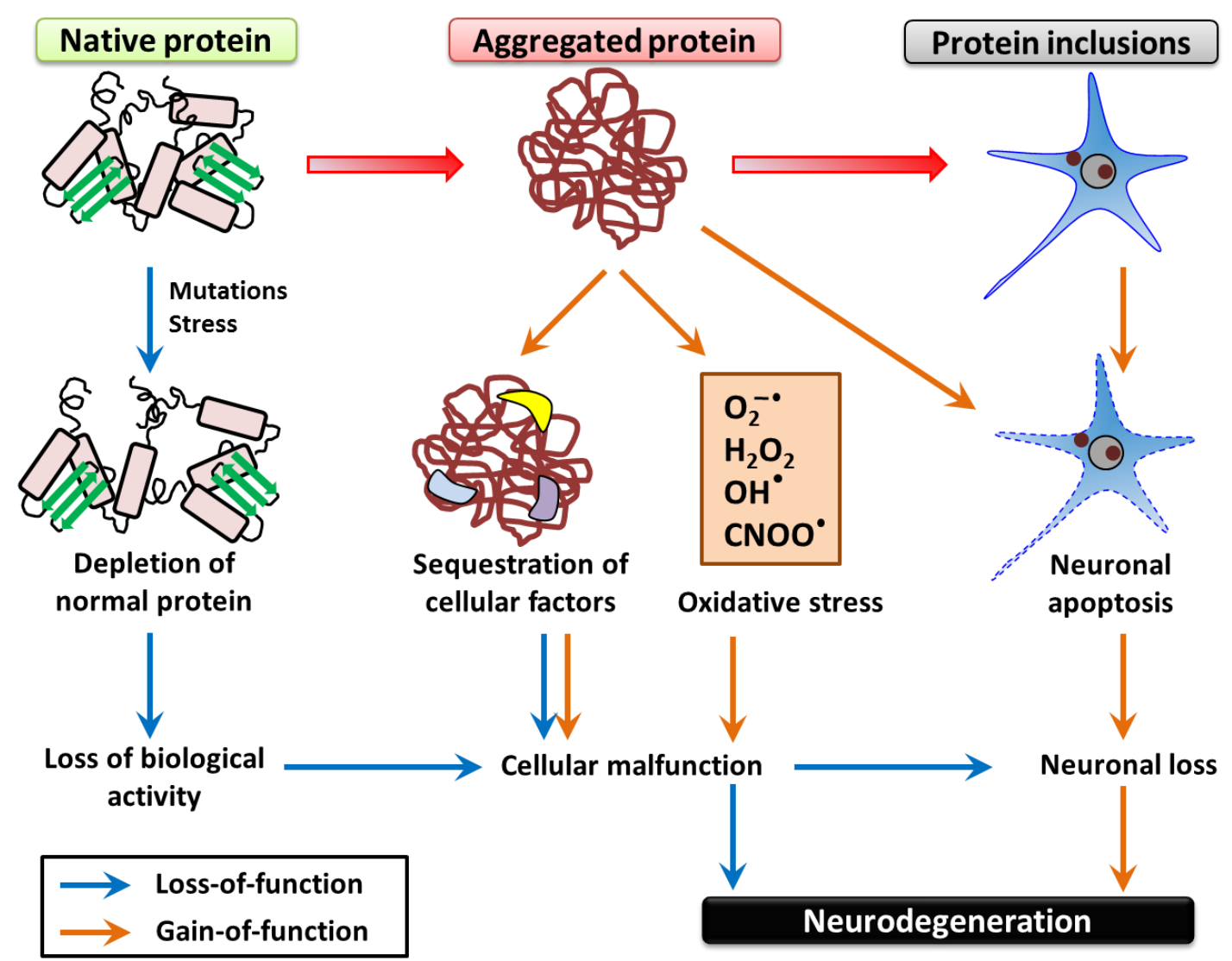

Figure 8: Models for the mechanism of neurodegeneration due to protein misfolding and aggregation.

Neuronal cell death in diseases like Huntington's disease and Alzheimer's disease can be contributed by both loss-of-function of native active proteins and/or gain-of-function by misfolded proteins. Mutations, translational errors and various kinds of stress can lead to protein unfolding and degradation which results in depletion of active functional molecules and hence loss-of-function phenotypes. Proteins can lose their structure and can engage in non-specific intermolecular interactions to form cytoplasmic and or intranuclear aggregates and inclusion bodies. Often, these aggregates, due to sticky exposed hydrophobic residues, can display gain-of-function phenotypes by either sequestering essential cellular factors or by causing oxidative stress.

Not only the misfolded proteins with gain-of-function phenotype can lead to pathological states and human diseases, but protein misfolding can also result in loss-of-function phenotypes (Winklhofer et al., 2008). For example, mutations in proteins such as p53 and VHL tumor suppressor cause enhanced degradation and subsequent decrease in the concentration of the active functional molecules may lead to tumor development (Scott and Frydman, 2003). It has also been shown that mutations in CFTR impair its correct folding and transport from the endoplasmic reticulum to the plasma membrane, causing loss of CFTR function and cystic 
fibrosis (Amaral, 2005). The following sub-section discusses in detail Huntington's disease as an example to understand the principles of gain-of-function and loss-of-function phenotypes.

\section{II.4.3.1. Huntington's Disease}

Huntington's disease (HD) is an autosomal dominant neurodegenerative disorder characterized by progressive motor dysfunction, cognitive decline and psychiatric disturbance due to neuronal cell death. It is caused by an expansion of trinucleotide CAG repeats in the first exon of the huntingtin gene (also called IT-15), which translates to an abnormally long polyglutamine (polyQ) stretch in the corresponding huntingtin protein (Htt) (The_Huntington's_Disease_Collaborative_Research_Group, 1993). Expansion of the polyQ stretch beyond 35-40 residues results in the formation of intranuclear inclusions in affected neurons (Davies et al., 1997; DiFiglia et al., 1997) and the propensity of Htt aggregation dramatically increases as the length of the polyQ stretch increases (Chen et al., 2002; Georgalis et al., 1998) (Figure 9). The mean age of onset of HD is 40-50 years, but expansion of the CAG repeat beyond 60 glutamines results in juvenile HD with more severe neuronal dysfunction. The length of the polyQ stretch in Htt is directly correlated to clinical severity of the disease and its penetrance in afflicted individuals (Duyao et al., 1993; Snell et al., 1993).



\section{Figure 9: HD onset as a function of CAG repeat length.}

$\mathrm{HD}$ is caused by an expansion of a CAG repeat in the first exon of the huntingtin gene. CAG trinucleotide encodes glutamine and expansion of glutamine residues beyond 35-40 in Htt protein results in HD.

The exact function of the ubiquitously expressed wild-type Htt protein is still elusive; however, certain studies report that $\mathrm{Htt}$ is essential for embryonic development and has an antiapoptotic role by blocking the activation of caspase-3 and caspase-9. Htt is also believed to play a role in vesicle transport and in regulating gene transcription and RNA trafficking (Cattaneo et 
al., 2005; Finkbeiner, 2011; Ross and Tabrizi, 2011; Zuccato et al., 2010). Therefore, HD involves both loss-of-function of the normal Htt that impairs its fundamental role in neuronal cells, and predominantly gain-of-function by the mutant Htt proteins that form amyloid-like inclusions (Scherzinger et al., 1997).

An intriguing aspect of Htt mediated cell toxicity is concerning the identification of the protein species that actually overrides the cellular proteostasis capacity and mapping of the interactome of mutant $\mathrm{Htt}$. A considerable body of experimental evidence suggests that formation of $\mathrm{Htt}$ inclusions is a protective mechanism, employed by cells to sequester potentially dangerous soluble oligomers of misfolded Htt (Arrasate et al., 2004; Bodner et al., 2006; Miller et al., 2010; Takahashi et al., 2008). This sequestration may reduce the number of exposed regions in mutant $\mathrm{Htt}$ that otherwise would titrate away cellular folding factors, putting the folding of the metastable proteome in jeopardy (Gidalevitz et al., 2006; Satyal et al., 2000). Another plausible cause of $\mathrm{Htt}$ toxicity may involve direct or indirect interference with the ubiquitin proteasome system as shown by the presence of ubiquitinated Htt and proteasome subunits in IBs (Chai et al., 1999; Jana et al., 2001; Mitra et al., 2009; Sieradzan et al., 1999; Venkatraman et al., 2004). Alternatively, Htt can lead to cell death by its combined effect on the cellular folding and degradation machinery as it may increase the load of endogenous aggregation prone proteins, resulting in loss-of-function phenotypes.

Consistent with these hypotheses, several studies have conducted genome wide screens in different model organisms such as transgenic D. melanogaster and $C$. elegans to identify genetic factors whose suppression or over-expression regulate the toxicity of mutant Htt (KazemiEsfarjani and Benzer, 2000; Nollen et al., 2004; Silva et al., 2011; Zhang et al., 2010). All these studies have identified genes belonging to distinct classes including genes involved in RNA metabolism (RNA pol II subunits, splicing factors), protein synthesis (ribosomes, initiation and elongation factors), protein folding (chaperones) and protein degradation (proteasome subunits and autophagy factors). The role of molecular chaperones, particularly Hsp70 and Hsp40, in suppressing $\mathrm{Htt}$ aggregation and its associated toxicity has been extensively elucidated using different experimental approaches (Hageman et al., 2010; Muchowski et al., 2000; Sakahira et al., 2002; Warrick et al., 1999; Wyttenbach et al., 2000). Besides molecular chaperones, cells 
have evolved distinct mechanisms to protect themselves from accumulation of toxic protein species. These mechanisms are discussed in the following sections.

\section{II.4.4. Cellular Defense Mechanisms against Protein Misfolding}

All cells have a remarkable capacity to buffer changes in the highly dynamic intracellular environment on exposure to proteotoxic stress. Given the fact that nearly $20-30 \%$ of all proteins in mammalian cells are intrinsically disordered (Dunker et al., 2008) and can engage in promiscuous molecular interactions (Vavouri et al., 2009), cells considerably invest in a number of protein quality control factors to prevent protein misfolding and aggregation. Among these factors, molecular chaperones and degradation machinery of proteostasis play an essential role.

Members of the chaperone family, besides participating in de novo folding of nascent chains, are also involved in refolding of misfolded protein species and re-solubilizing aggregates. The degradation machinery, comprising the ubiquitin proteasome system and autophagy components, ensures the timely removal of irreparable and terminally misfolded proteins. Since both chaperone mediated refolding and degradation are ATP dependent processes, from a thermodynamic perspective refolding is preferred over degradation. This kind of cellular triage decision whether to refold or to degrade proteins is critically dependent on co-chaperones like CHIP and E3 ubiquitin ligases. Cells may also sequester and direct potentially deleterious protein species to specific cellular sites in order to avoid toxic effects of protein aggregation. Such deposition sites could be aggresomes (Johnston et al., 1998; Kopito, 2000) or recently identified juxtanuclear quality-control compartment (JUNQ) and insoluble protein deposit (IPOD) sites in yeast and higher eukaryotes (Kaganovich et al., 2008). This organization would probably allow cells to facilitate the efficient clearance of aggregate in subsequent steps of either disaggregation or degradation.

In the following sub-sections, the role of molecular chaperones in refolding and disaggregation and the role of the degradation machinery in protecting cells are briefly discussed.

\section{II.4.4.1. Chaperone Mediated Refolding and Disaggregation}

Using model proteins like $\beta$-galactosidase and firefly luciferase, many studies have addressed the role of the Hsp70 (DnaK)-Hsp40 (DnaJ)-Hsp110 (GrpE) system (also called KJE system) in re-activation of stress denatured proteins both in vitro and in vivo (Ben-Zvi et al., 
2004; Goloubinoff et al., 1999; Pinto et al., 1991; Schroder et al., 1993). The refolding reaction is generally slow and is strictly dependent on ATP hydrolysis. For example, it has been demonstrated that one Hsp70 molecule requires five ATP molecules to effectively unfold a single molecule of misfolded luciferase into an intermediate precursor, which can spontaneously refold into the native state upon chaperone dissociation (Sharma et al., 2010). It is not only the KJE system that is capable of refolding stress denatured proteins, but other chaperones such as Hsp90 in cooperation with Hsp70 also participate in recovery of cells by promoting either refolding or degradation of misfolded proteins (Schneider et al., 1996; Schumacher et al., 1996; Thulasiraman and Matts, 1996).

Since protein misfolding leads to aggregation, cells have evolved a mechanism to disaggregate IBs for efficient resolubilization and refolding by chaperones in subsequent steps. The phenomenon of disaggregation was first reported in S. cerevisiae where heat denatured proteins can be efficiently reactivated by the cooperative action of Hsp70-Hsp40 and the oligomeric, ring forming AAA+ ATPase chaperone Hsp104 (Glover and Lindquist, 1998; Parsell et al., 1994). Soon after the discovery of Hsp104 disaggregase in yeast, an orthologous protein called $\mathrm{ClpB}$ was shown to possess disaggregation activity in E. coli and in chloroplasts and mitochondria of higher eukaryotes (Mogk et al., 1999). Several in vitro studies have shown that the Hsp104 or ClpB system alone has little or no disaggregation activity and requires collaboration with the Hsp70 system for effective disaggregation (Glover and Lindquist, 1998; Goloubinoff et al., 1999).

Hsp104 together with the Hsp70 system forms a bi-chaperone system, whose induction during lethal stress enables cells to transiently acquire thermotolerance against subsequent stress (Zietkiewicz et al., 2004). Though the precise mechanism of disaggregation is still elusive, some evidence suggests that Hsp70-Hsp40 remodels protein aggregates and allows the transfer of aggregated polypeptides to the substrate processing pore of Hsp104 (Figure 10). Hsp104, in an ATP dependent process, then exerts a threading or pulling activity to facilitate extraction of the misfolded polypeptides from the aggregates (Liberek et al., 2008; Lum et al., 2004; Tyedmers et al., 2010). Once inside the Hsp104 cylinder, the polypeptide is disentangled to soluble form. Upon exit from the Hsp104, the solubilized protein can re-enter chaperone mediated refolding cycles (Figure 10). There are only few reports about a disaggregation activity in mammalian 
cells (Yamamoto et al., 2000) and in C. elegans (Cohen et al., 2006). Recently, in a cell-free system, Hsp110 (Apg-2) has been identified as a mammalian disaggregase that in conjunction with Hsp70-Hsp40 can catalyze protein disaggregation and reactivation (Shorter, 2011).

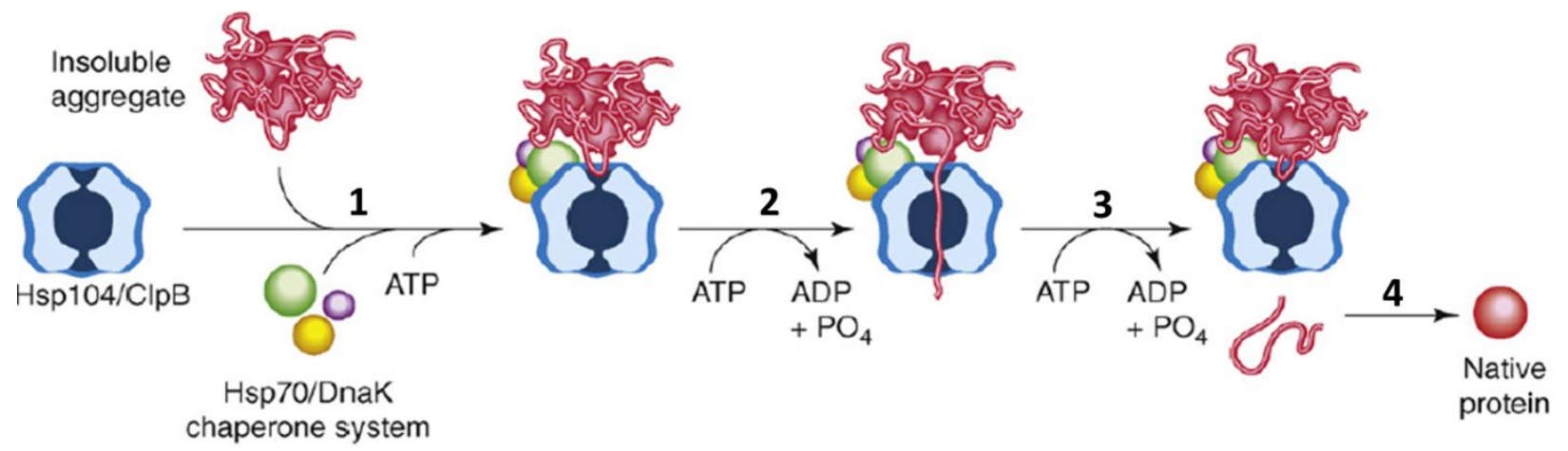

Figure 10: Disaggregation mediated by Hsp104 and ClpB in cooperation with the Hsp70 chaperone system.

Protein disaggregation in cells is carried out by AAA+ proteins, Hsp104 in S. cerevisiae or ClpB in E. coli and occurs in multiple steps. (1) The Hsp70 (DnaK)-Hsp40 (DnaJ) chaperone system remodels protein aggregates in an ATP dependent process. (2) The Hsp70-Hsp40 system then assists in presenting substrate to the translocation pore of $\mathrm{Hsp} 104 / \mathrm{ClpB}$ where polypeptide is pulled at the cost of ATP hydrolysis into their catalytic core. (3) The polypeptide is then disentangled to a soluble form and (4) released either to refold spontaneously or with assistance from additional molecular chaperones. Adapted from (Doyle and Wickner, 2009).

The Hsp70-Hsp40-Hsp104 mediated protein disaggregation process is further enhanced by small HSPs such as Hsp27 that directly interact and bind to aggregating proteins. This interaction of small HSPs with aggregates may shield the non-native surfaces and hence serves to protect the misfolded proteins from proteases. In an ATP independent process, an aspect that is remarkably different from other chaperones (except TF), small HSPs induce changes in the physiochemical properties of misfolded polypeptides in the aggregates. This presumably facilitates the disaggregation machinery to effectively separate and unfold the individual polypeptides in the aggregates (Haslbeck, 2002; Haslbeck et al., 2005; Mogk et al., 2003).

\section{II.4.4.2. The Degradation Arm of Proteostasis}

The degradation machinery of a cell consisting of ubiquitin-proteasome system (UPS) and autophagy serves to remove irreparable misfolded proteins and aggregates. Both UPS and autophagy have been shown to play a protective role in mitigating the toxic effects of proteins 
responsible for protein conformational diseases (Hara et al., 2006; Komatsu et al., 2006; Ravikumar et al., 2002; Rubinsztein, 2006; Webb et al., 2003). The following sub-sections discuss in detail the main components and features of UPS and autophagy in protein quality control.

\section{II.4.4.2.1. The Ubiquitin-Proteasome System (UPS)}

The UPS is constituted by two essential protein components, ubiquitin and the proteasome. Cells must be able to faithfully distinguish proteins destined for degradation from the rest of its constituent proteome in order to avoid the widespread and unregulated breakdown of proteins. Therefore, cells have ingeniously designed a mechanism that involves the covalent tagging of a 76 residues protein called ubiquitin to proteasome substrates (Hershko and Ciechanover, 1998). This process of covalent attachment of ubiquitin is highly specific and follows an ATP dependent enzymatic cascade through different enzymes E1, E2 and E3 (Ciechanover, 1998) (Figure 11a).

Briefly, in the first step, ubiquitin is activated by an E1 ubiquitin-activating enzyme that adenylates the C-terminal carboxyl group of ubiquitin using ATP. This activation step is followed by the formation of a thioester intermediate between conserved Cys residue of the E1 and the C-terminus of ubiquitin. Ubiquitin is then shuttled from the E1 to a conserved Cys residue of the ubiquitin-conjugating enzyme E2. The final step in protein ubiquitination involves the transfer of ubiquitin from the E2 to the target protein via an isopeptide bond between the Cterminal carboxyl group of ubiquitin Gly76 and the $\varepsilon$-amino group of a Lys residue on the target protein. E3 ubiquitin ligases, together with their ancillary factors, bind to the substrates and assist the E2 enzymes in transferring the ubiquitin moiety. Thus, E3 ligases bind to the substrates prior to ubiquitin conjugation and many are highly specific and selective for different substrates. Upon conjugation of a single ubiquitin to the target protein, additional isopeptide bonds are sequentially formed between the C-terminal Gly76 of another incoming ubiquitin and one of the seven Lys residues (generally Lys48) of previously conjugated ubiquitin on the modified protein to generate polyubiquitin chains (Finley et al., 1994; Pickart, 2000). A polyubiquitin conjugated protein is subsequently targeted to the proteasome for its degradation. 

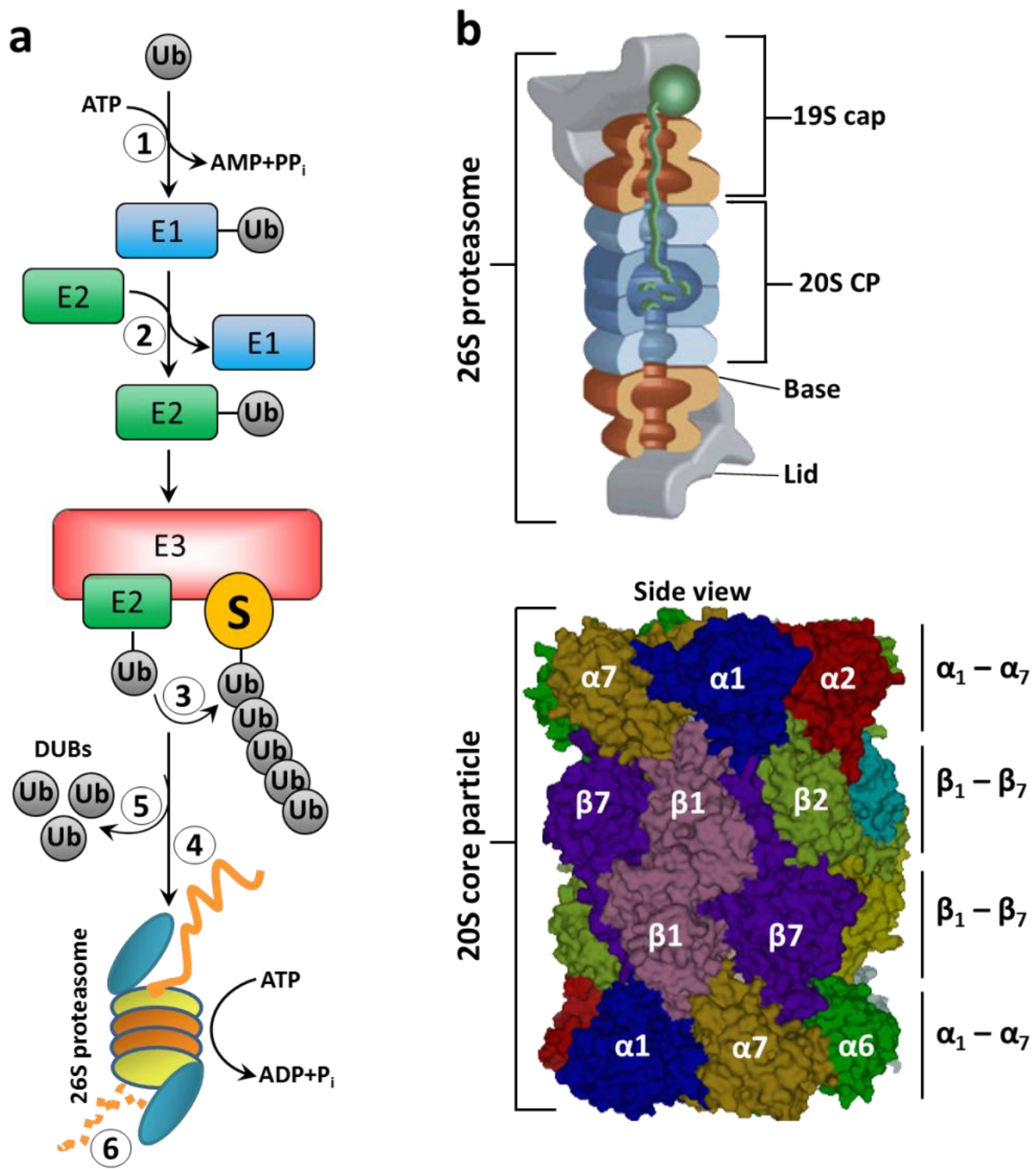

Figure 11: The ubiquitin-proteasome system (UPS).

(a) Proteasome-dependent degradation of ubiquitinated substrates. Ubiquitination and degradation of substrate by proteasome involves an enzymatic cascade and occurs through multiple steps. (1) Activation of ubiquitin by E1 ubiquitin-activating enzyme. (2) Activated ubiquitin is then transferred to E2 conjugating enzyme. (3) E3 ubiquitin ligase binds the protein substrate and the E2 enzyme which carries an activated ubiquitin. The E3 enzyme then catalyzes the transfer of the ubiquitin moiety from the E2 enzyme to the protein substrate. This step is repeated multiple times to generate a polyubiquitin chain on the substrate protein. (4) The polyubiquitinated substrate is subsequently targeted to the $26 \mathrm{~S}$ proteasome for degradation. (5) The ubiquitin pool is restored by the action of deubiquitinating enzymes that remove polyubiquitin chains from the substrate (6) The substrate is then channeled through the catalytic core of the proteasome where it is cleaved into short peptides. (b) Structure of eukaryotic $26 \mathrm{~S}$ proteasome. The $26 \mathrm{~S}$ proteasome consists of two subcomplexes; the $20 \mathrm{~S}$ core particle and the $19 \mathrm{~S}$ regulatory particle. The $20 \mathrm{~S}$ core particle is a barrel-shaped structure made of four stacked rings, two identical outer $\alpha$-rings and two identical inner $\beta$-rings. Each $\alpha$ and $\beta$ ring consists of 7 subunits which are arranged in $\alpha_{7} \beta_{7} \beta_{7} \alpha_{7}$ symmetry in the 20S complex. Adapted from (Ciechanover, 2005; Maupin-Furlow, 2012). 
The proteasome is a large, self-compartmentalized nanomachine that harbors different proteolytic subunits and is evolutionary conserved across the three domains of life (Baumeister et al., 1998; Maupin-Furlow, 2012). The self-assembly of these proteolytic subunits to form a functionally active proteasome enables the cells to localize them to different cellular locations in the cytosol or in the nucleus, depending on the need. This adds another layer of complexity to the spatial-temporal regulation of proteolysis, concomitant with the physiological and developmental requisite of the cells. Similar to molecular chaperones, the proteasome also forms a central hub of the proteostasis network. It regulates essential cellular processes such as cell division, DNA repair, apoptosis etc. by controlling the turnover of regulatory proteins, including cyclins and kinases.

The functional eukaryotic proteasome (26S) consists of a barrel-shaped 20S core particle capped on each side by 19S regulatory complexes (Figure 11b). The 20S core particle is cylindrical and is composed of structurally similar $\alpha$ and $\beta$ subunits, stacked upon each other to form four heptameric rings (Groll et al., 1997). The outermost rings contain $\alpha$ subunits while the inner rings are made of $\beta$ subunits, assembled in $\alpha_{7} \beta_{7} \beta_{7} \alpha_{7}$ symmetry. The amino-terminal threonine residues of $\beta$ subunits form the catalytic active sites for proteolysis. In eukaryotes, only three of the seven $\beta$ subunits in a single ring are proteolytically active. This results in a total number of 6 active sites in the 20S core particle (Baumeister et al., 1998; Voges et al., 1999). Using fluorogenic peptide substrates, studies have shown that eukaryotic proteasomes possess three major peptidase activities: (i) chymotrypsin-like activity on $\beta 5$ subunits, which cleaves after hydrophobic residues, (ii) trypsin-like activity on $\beta 2$ subunits, which cleaves after basic residues, and (iii) endopeptidase Glu-C like activity on $\beta 1$ subunits, which cleaves after acidic residues. Additionally, mammalian proteasomes can also cleave after branched chain residues and between small neutral acids (Cardozo, 1993). The structural and functional information of proteasomes has allowed the design of small molecules to inhibit its proteolytic activity. These inhibitors, in most of the cases, block the chymotrypsin-like activity of $\beta$ subunits either reversibly, such as MG132, or irreversibly such as lactacystin and epoxomicin (Kisselev and Goldberg, 2001; Myung et al., 2001).

The openings at the end of the 20S core particle is gated by the N-terminal tails of $\alpha$ subunits which may play a role in regulating the translocation of substrates through the proteolytic channel and may participate in mediating the interaction of $20 \mathrm{~S}$ core particle with 
$19 \mathrm{~S}$ regulatory complexes. The $19 \mathrm{~S}$ regulatory particle is composed of a lid and a base subcomplex. The base is made of six different AAA+ family ATPase subunits (Rpt1-6) and three non-ATPase subunits (Rpn1, Rpn2 and Rpn13). The lid harbors nine other Rpn subunits (Rpn3, Rpn5-9, Rpn11-12 and Rpn15) (Tomko and Hochstrasser, 2011; Wolf and Hilt, 2004). The AAA+ proteins (Rpt1-6) of 19S regulatory complexes selectively bind and unfold substrate proteins, open the gate of $20 \mathrm{~S}$ core particle and facilitate the vectorial translocation of unfolded substrates into the catalytic chamber for proteolysis (Braun et al., 1999; Smith et al., 2005). Proteasomal subunits Rpn10 and Rpn13 bind to polyubiquitin chains with very high affinity and tetra-ubiquitin chains have been shown to be a good signal for degradation (Thrower et al., 2000). Upon stable interaction of the polyubiquitin tagged polypeptide with the proteasome, the deubiquitylating enzymes (DUBs) cleave off the ubiquitin chain and restore the ubiquitin pool.

The proteasome is highly processive in nature and generally generates small peptides of around 7-9 residues that can be further cleaved into individual amino acids by cytosolic proteases. Besides recycling the amino acids for protein biogenesis, the proteasome also has an essential role in generating immunocompetent peptides to be displayed by the MHC class I complex.

\section{II.4.4.2.2. Autophagy Mediated Degradation}

Autophagy is defined as a process by which intracellular cytosolic material, including proteins, organelles and pathogens are degraded inside the acidic lumen of lysosomes. Therefore, autophagy provides an alternative protein quality control pathway for the destruction of irreparable misfolded proteins, both soluble forms and aggregates. Autophagy is activated under different physiological stress conditions primarily during starvation and oxidative stress (Cuervo et al., 1995; Kiffin et al., 2004). Thus, like the UPS, autophagy helps in buffering the effects of proteotoxic insults by degrading misfolded proteins and coordinates with the chaperone network to fine tune the proteostasis balance between folded and unfolded proteome (Wong and Cuervo, 2010).

Four types of autophagy have been shown to co-exist in most cells. While macroautophagy and microautophagy are non-selective for their targets, chaperone mediated autophagy (CMA) (Cuervo, 2011) and chaperone-assisted selective autophagy (CASA) (Arndt et al., 2007; Kettern et al., 2010) are highly selective forms of autophagy. Macroautophagy is a 
process where cellular organelles like mitochondria (mitophagy), giant proteins like ribosomes (ribophagy), lipid droplets (lipophagy) and protein aggregates are secluded 'in bulk' from the cytosol by a de novo formed double membrane vesicle called autophagosome (Mizushima et al., 2002). The autophagosomes, in a microtubule-dynein dependent process fuse with lysosomes. The acidic environment and hydrolases (proteases, lipases, glycosidases etc.) in the lumen of lysosomes then digest the cargo. Microautophagy, on the other hand, involves invagination of the lysosomal membrane to sequester cytosolic proteins (in a non-selective manner) in a vesicle that later pinches off and release its content in the lysosomal lumen for degradation.

During CMA, soluble cytosolic proteins (often misfolded) and aggregates displaying a pentapeptide sequence similar to KFERQ are recognized and bound by Hsc70 and its cochaperones, which deliver the substrate to the monomeric form of LAMP2A receptor (lysosome associated membrane protein $2 \mathrm{~A})$ at the lysosome membrane. The docking of substratechaperone complex onto the cytosolic tail of LAMP2A receptor promotes multimerization of LAMP2A into a high molecular weight complex. This process then causes unfolding of the substrate and its translocation across the lysosomal membrane with aid from Hsc70 in the lumen of lysosome. Inside the lumen, the translocation complex disassembles and the polypeptides are degraded by the processive action of endo- and exo-proteases (Cuervo, 2011).

Unlike CMA, which does not involve vesicle formation or invagination, CASA involves selective engulfment of the substrates by an autophagic isolated membrane leading to lysosomal degradation (Arndt et al., 2007; Kettern et al., 2010). In CASA, ubiquitination serves as an initial degradation signal, comparable to the proteasomal degradation. The ubiquitinated substrates are recognized by the Hsc70-BAG-3 complex that facilitates the interaction of the substrate with the adaptor proteins p62 or NBR1 on the autophagosome membrane. This process directs diverse chaperone clients towards the lysosomal degradation pathway. The interaction of the cochaperone BAG-3 with Hsc70 directs ubiquitinated substrates to the autophagy pathway, while the interaction of BAG-1 with Hsc70 promotes UPS mediated degradation.

Several differences in degradation mechanisms exist between the UPS and autophagy. For example, in the UPS, proteolysis is selective, regulated and fast whereas in autophagy it is relatively slow and the exact mechanism from initiation of signal for autophagy to protein turnover is still not very clear. Also, unlike UPS, autophagy plays an essential role in recycling 
precursor molecules (amino acids, fatty acids, sugar monomers etc.) by eliminating the nonessential pool of macromolecules particularly during starvation.

\section{II.4.5. Small Molecule Regulators of Proteostasis}

The advancement in x-ray crystallography has enabled the detailed understanding of the structures of various protein molecules and has allowed correlating the structural changes in proteins upon their interaction with different molecules under different physiological conditions. Hence, it is now possible to rationally design chemical compounds that can reversibly or irreversibly bind to proteins at specific sites to alter their structural and functional properties. Such chemically synthesized compounds, so called small molecule regulators, have also been designed to restore deficiencies in proteostasis by modulating the key proteins of the proteostasis network. Since the proteostasis network encompasses a wide range of key proteins involved in different steps in protein biogenesis, maintenance (folding and refolding), trafficking and degradation, both activators and inhibitors have been screened to affect each of these individual steps. A combination of chemical and biological approaches can be used to rebalance the cellular proteostasis capacity under stress conditions and therefore hold a great therapeutic potential to treat several human diseases including protein conformational disorders and cancer (Lindquist and Kelly, 2011; Mu et al., 2008; Powers et al., 2009).

The small molecule regulators of proteostasis can be categorized into several groups depending on their mode of action and their targets in the proteostasis network. The first group consists of small molecules that directly activate or inhibit HSF1 mediated heat shock response. Since induction of various HSPs is dependent on the heat shock response, these proteostasis regulators (PR) can change the folding landscape of cells under stress conditions. Indeed, results from compound screens have identified several PR that induce or prevent the heat shock response (Calamini et al., 2012; Westerheide and Morimoto, 2005). For instance, celastrol has been identified as an activator of heat shock response (Trott et al., 2008; Westerheide et al., 2004) and has been shown to protect neuronal cells against toxic effects of mutant Htt (Wang et al., 2005; Zhang and Sarge, 2007) or mutant SOD1 (Kiaei et al., 2005). Similarly, triptolide has been identified as an inhibitor of HSF1 and hence of heat shock response (Westerheide et al., 2006) and has been demonstrated to induce apoptosis in pancreatic cancer cells by reducing Hsp70 levels (Phillips et al., 2007). 
The second group of molecules acts by directly interacting with chaperones or the proteasome. For example, geldanamycin and its derivatives have been documented to inhibit Hsp90 and prevent the maturation of its client proteins. Likewise, MG132 inhibits the proteasome and increases the load of misfolded proteins in the cells. Therefore, both geldnamycin and MG132 at optimal concentrations are suitable drug candidates against transformed cells like certain types of tumor (Kisselev and Goldberg, 2001; Whitesell and Lindquist, 2005). Both geldanamycin and MG132 treatment leads to the accumulation of misfolded proteins and as a result the chaperones (mainly Hsp70 and Hsp90) dissociate from HSF1. This leads to the activation of HSF1. Therefore, both these compounds also induce the heat shock response and increase the cellular concentrations of HSPs (Bush et al., 1997; Sittler et al., 2001). This effect could be beneficial in preventing the aggregation of proteins such as mutant Htt (Sittler et al., 2001).

The next group of small molecules includes compounds that act as chemical chaperones by mimicking the role of a co-chaperone or as pharmacological chaperones (PC) by directly interacting with the substrate protein and helping it in acquiring its native folded state. For example, an unbiased screen has identified a compound $115-7 \mathrm{c}$ which has been shown to act as an artificial co-chaperone for Hsp70. This compound stimulates ATPase and folding cycle of Hsp70 (DnaK) and can partially compensate for a Hsp40 loss-of-function phenotype in yeast (Wisen et al., 2010). In a recent study, it was shown that administration of PR such as celastrol or MG132 increase the unfolded protein response (UPR) resulting in the coordinated transcription and translation of chaperones and folding catalysts that promote folding of mutant enzyme in the ER and prevent it from misfolding/aggregation. The co-administration of PC stabilizes the folded state of mutant enzyme and synergizes with PR to effectively rescue the loss-of-function phenotype of the enzyme (Mu et al., 2008). Therefore, it seems possible that a combination therapy of highly specific PR and PC can be useful to treat protein conformational disorders.

\section{II.4.6. Proteostasis in Aging}

Aging, a unidirectional and complex phenomenon from birth to death of an organism, is associated with progressive decline in the physiological functions and the compromised ability of cells to mount a stress response. At the molecular level, aging is characterized by the culmination of multiple effects on the structural and functional properties of macromolecules. There is a 
gradual accumulation of deleterious modifications in nucleic acids, proteins, carbohydrates and lipids that are no longer repairable by cells, leading to loss- or gain-of-function phenotypes and hence age related diseases (Kenyon, 2005).
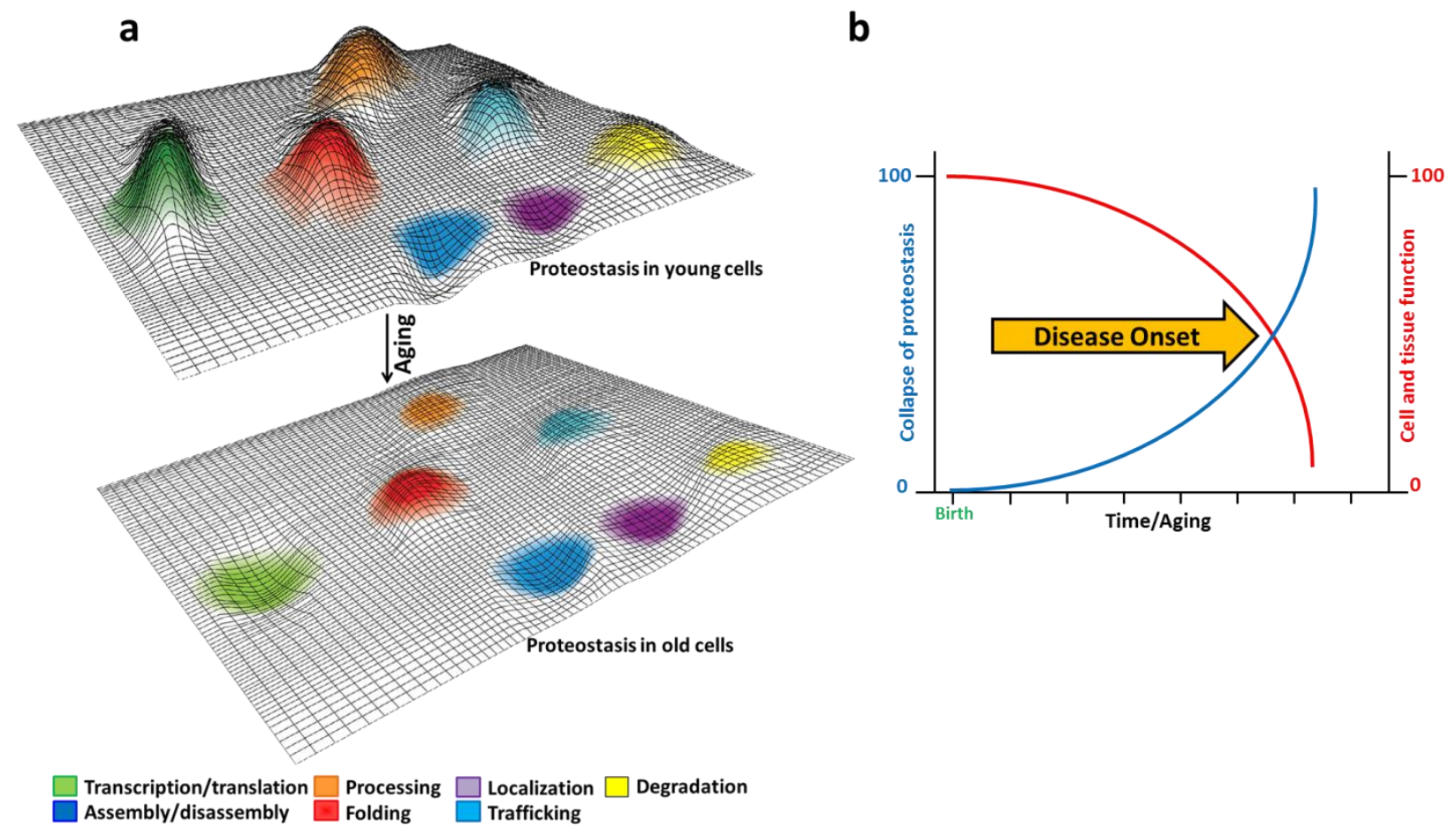

\section{Figure 12: Gradual collapse of proteostasis during aging.}

(a) An arbitrary representation of the major components of the proteostasis network on a global landscape in two different conditions, young and old. General activity for components involved in transcription, translation, folding, trafficking, processing, assembly/disassembly, localization and degradation are depicted as nodes in which amplitude of node reflects strength of homeostatic activity. A general imbalance in the activities of the major components of the proteostasis network is suggested to ensue during aging. Adapted from (Douglas and Dillin, 2010). (b) An inverse correlation exists between failure of cellular proteostasis and loss of cellular function during aging. The progressive loss of proteostasis capacity during aging results in decline of cell and tissue function and consequently predisposes individuals to age-related disorders such as HD.

During aging, there is a progressive decline in the efficiency of protein quality control mechanisms (Gidalevitz et al., 2006; Morimoto, 2008) (Figure 12). This deficit in the proteostasis capacity predisposes individuals to many late-age onset diseases including neurodegenerative diseases such as AD, PD and HD and other maladies like diabetes type II, myopathies etc. (Kikis et al., 2010). The role of various signaling pathways and stress responses in aging is exemplified from recent findings obtained from C. elegans and D. melanogaster. For 
example, the reduced activity of the insulin/IGF-1-like signaling (IIS) pathway in mediating lifespan extension in various organisms has been well demonstrated (Kenyon, 2010). In $C$. elegans, it has been reported that reduced IIS is beneficial for cells as it protects them from toxicity by protein aggregates and HSF1 is required for the IIS pathway to extend lifespan (Cohen et al., 2006; Cohen et al., 2009; Morley and Morimoto, 2004).

The essential role of molecular chaperones in maintaining proteostasis in aging has been extensively elucidated by several studies. For instance, over-expression of small heat shock proteins is sufficient to extend lifespan in C. elegans and in D. melanogaster (Morley and Morimoto, 2004; Morrow et al., 2004). Similarly, induction of molecular chaperones by either over-expressing HSF1 (Hsu et al., 2003; Morley and Morimoto, 2004) or by a sublethal dose of heat shock significantly extends the lifespan in model organisms and induces resistance against subsequent proteotoxic stress (this process is called as hormesis) (Cypser et al., 2006; Olsen et al., 2006). Likewise, several studies have shown that the inability of cells to mount an adequate stress response against misfolded proteins results in low Hsp70 proteins levels and augments the aging process (Bonelli et al., 1999; Fargnoli et al., 1990; Gutsmann-Conrad et al., 1998). This affect is presumably due to an increase load of misfolded proteins on the proteostasis machinery that directly or indirectly affects the folding of the metastable proteome of a cell, resulting in widespread failure of diverse pathways (Ben-Zvi et al., 2009).

With aging, there is also a gradual decline in the turnover of proteins by UPS and CMA in a variety of tissues which further enhances the aggregation of proteins in cells (Cuervo and Dice, 2000; David et al., 2010; Tonoki et al., 2009). The decrease in UPS activity can be due to multiple mechanisms like a decrease in the expression levels of proteasomal subunits or by inhibition of the proteasome by oxidized and damaged proteins. In a transgenic mouse model it has been recently shown that by modulating the amount of LAMP2A proteins the CMA activity can be maintained until advanced age (Zhang and Cuervo, 2008). Therefore, up-regulation of UPS and autophagy by small molecules or by genetic modulation can extend the lifespan of an organism.

Beside defects in cytosolic stress response and protein quality control mechanisms, other factors such as oxidative stress, improper UPR in the ER and mitochondria, or failure of DNA repair mechanisms all contribute to aging. The downstream effect of all these factors results an 
unstable environment in the cells, not capable of sustaining homeostasis under basal or elevated stress conditions (Kourtis and Tavernarakis, 2011; Taylor and Dillin, 2011). Thus, aging is a process exemplified by a cumulative failure of cellular homeostasis due to a multitude of diverse and possibly inter-connected factors.

\section{II.4.7. Sensors of Proteostasis}

Research in the field of protein folding and misfolding has only begun to investigate how different components of the proteostasis collaborate with each other to shape the structural and functional properties of protein molecules. Our understanding of how different protein quality control pathways are functionally integrated with each other and with the stress responses is still limited. Several studies have given insights into the molecular mechanisms responsible for proteostasis collapse in protein conformational diseases and during aging. However, several questions still remain to be investigated such as the identification of toxic species in these diseases and the status of proteostasis in different organelles and in different tissues. In order to screen large numbers of small molecules as therapeutic drugs for diseases of proteostasis deficiency, it becomes essential to have a suitable reporter to rapidly measure proteostasis in vivo. The fundamental understanding of the function and regulation of the proteostasis network requires molecular tools or sensors to measure its status in different physiological conditions (proteostasis sensors).

Pioneering studies in $C$. elegans have established the use of temperature sensitive mutants of endogenous proteins such as $\alpha$-paramyosin, dynamin, $\alpha$-myosin and other proteins as sensors of proteostasis (Ben-Zvi et al., 2009; Gidalevitz et al., 2006). These mutant proteins, at permissive temperature $\left(15^{\circ} \mathrm{C}\right)$ are correctly folded and stable, but at higher temperature $\left(25^{\circ} \mathrm{C}\right)$ rapidly unfold and aggregate. Upon expression of mutant Htt protein or during normal aging, these mutant proteins start aggregating even at the permissive temperature. Though useful in understanding dysregulation of proteostasis in C. elegans, these proteins have some limitations as proteostasis sensors. Since these proteins are endogenously expressed in cells, their compromised folding may lead to loss-of-function effects and reduced viability. Moreover, at restrictive temperature, these mutant proteins can themselves cause some intrinsic stress to tissues and therefore can contribute to the observed effects. These mutant proteins, being tissuespecific, limit the scope of performing comparative analyses of the effect of aging or any other 
physiological stress on the activity of the proteostasis network in different cell types, tissues and model organisms.

Apart from proteins, the small organic dye ANS (1-anilino-8-naphthalenesulfonate) has been used as a sensor to detect protein misfolding in vivo (Hadley et al., 2011). This dye has a relatively low fluorescence in aqueous solution which increases significantly upon binding of the dye to a hydrophobic surface. Therefore, any stress such as heat stress or UPS inhibition that results in protein misfolding enhances the ANS fluorescence compared to normal conditions. However, the use of ANS as a sensor has several disadvantages. For example, it cannot be used to measure the folding status of proteins in cells in the presence of amyloid proteins such as Htt or $\alpha$-synuclein that will bind to ANS more strongly. Moreover, ANS can also bind to lipid droplets and membranes in cells and therefore a change either in the concentration or composition of lipids during stress may change ANS fluorescence which would be difficult to correlate with the changes occurring at the protein level.

Many other studies have used different kinds of reporters to measure changes in individual components of the proteostasis network. For example, green fluorescent protein fused with a short degron sequence at its C-terminus (GFP-CL1) is a good substrate of UPS and hence it is rapidly degraded in cells under normal conditions. However, in the presence of aggregation prone proteins that inhibit UPS function, the cellular concentration of GFP-CL1 fusion protein increases and can be easily monitored (Bence et al., 2001; Nonaka and Hasegawa, 2009). Since GFP-CL1 only reports the changes in UPS activity and cannot assess the changes in other components of the proteostasis network, it is not an ideal sensor to measure cellular stress. 


\section{Aim of the Study}

One of the biggest problems in the field of protein folding and misfolding is to understand how changes in the proteostasis machinery affect the dynamics and regulation of proteins. The comprehensive understanding of the proteostasis network and its status during disease or aging relies on the development of molecular tools or reporters that can accurately and rapidly measure the cellular proteostasis capacity in different conditions. All previously described sensors have some limitations and are therefore not optimal to assess the proteostasis status in a wide range of experimental systems, including cell and organism models of stress, neurodegenerative disease and aging.

To circumvent these limitations, the objectives covered in this thesis are the following.

1. To design sensor proteins that can provide a robust, generic and quantitative readout of proteostasis in vivo. We selected American firefly (Photinus pyralis) luciferase (Fluc) as a model protein and designed site-specific mutants to serve as sensitive reporters of proteostasis capacity.

2. To characterize the Fluc mutants in vitro using rabbit reticulocyte lysate (RRL) and to assess their sensitivity in a tissue culture model system under different stress conditions.

3. To demonstrate the applications of the Fluc-based sensors in C. elegans and to investigate the tissue specific differences (body wall muscle cells and neuronal cells) in maintaining proteostasis during heat stress and aging.

4. To selectively target Fluc-based sensor proteins to the nucleus and the cytosol of cells to measure and compare inter-compartmental proteostasis capacity. 


\section{Materials and Methods}

\section{IV.1. Materials}

\section{IV.1.1. Chemicals}

10X DNA Loading Buffer

17-AAG

1-Propanol p.a.

2-Phenylbenzothiazole (PBT)

Acetic acid

Acetone

Acrylamide : Bisacrylamide; $37.5: 1 ; 30 \%$

Agar

Agarose LE

Albumin bovine Fraction V

Ammonium Persulfate

Ampicillin-Natriumsalz

Bacto-Agar

Bacto-Peptone

Bacto-Tryptone

Bacto-Yeast Extract

Bio-Rad Protein Assay

Bis-Tris

Bromophenol Blue Sodium Salt
Fermentas (St. Leon-Rot, Germany)

Enzo Life Sciences (Lorrach, Germany)

Merck (Darmstadt, Germany)

Sigma (Deisenhofen, Germany)

Merck (Darmstadt, Germany)

Sigma (Deisenhofen, Germany)

Serva (Heidelberg, Germany)

Fluka (Buchs, Switzerland)

Biozym (Hess. Oldendorf, Germany)

Serva (Heidelberg, Germany)

Sigma (Deisenhofen, Germany)

Merck (Darmstadt, Germany)

Difco (Detroit, USA)

Difco (Detroit, USA)

Difco (Detroit, USA)

Difco (Detroit, USA)

Bio-Rad (Munich, Germany)

Fluka (Buchs, Switzerland)

Sigma (Deisenhofen, Germany) 
BSA Standard

Calcium Chloride Dihydrate

Cholesterol

Celastrol

Complete Protease Inhibitor Cocktail

Cycloheximide

DAPI

DharmaFECT Transfection Reagent

Dimethylsulphoxide (DMSO)

di-Potassium Hydrogen Phosphate

di-Sodium Hydrogen Phosphate

Dithiothreitol (DTT)

dNTP Set

Dulbecco's MEM

Dulbecco's MEM without phenol red

ECL Plus Detection Kit

Endotoxin Free Water

Ethanol Absolute

Fetal Bovine Serum (FBS)

Fluorescent Mounting Medium

GeneRuler 100bp DNA ladder

GeneRuler 1kb DNA ladder

Geneticin (G418)/Neomycin
Pierce (Rockford, USA)

Sigma (Deisenhofen, Germany)

Fluka (Buchs, Switzerland)

Calbiochem (La Jolla, USA)

Roche (Basel, Switzerland)

Sigma (Deisenhofen, Germany)

Invitrogen (Carlsbad, USA)

Thermo Scientific (Colorado, USA)

Sigma (Deisenhofen, Germany)

Carl Roth GmbH (Karlsruhe, Germany)

Carl Roth GmbH (Karlsruhe, Germany)

Sigma (Deisenhofen, Germany)

Metabion (Martinsried, Germany)

Biochrom AG (Berlin, Germany)

Biochrom AG (Berlin, Germany)

Millipore (MA, USA)

Sigma (Deisenhofen, Germany)

Merck (Darmstadt, Germany)

Gibco (Paisley, UK)

DAKO (Hamburg, Germany)

Fermentas (St. Leon-Rot, Germany)

Fermentas (St. Leon-Rot, Germany)

PAA (Pasching, Austria) 
Glycerin/Glycerol

Glycine

Guanidinium Chloride

Hygromycin B

Immersion oil

Kanamycin

Leptomycin B

L-Glutamine

Lipofectamine 2000 reagent

Lipofectamine PLUS reagent

Lipofectamine RNAiMAX reagent

Magnesium Sulphate

Manganese Chloride Dihydrate

Methanol p.a.

MG132

MOPS

N,N,N',N'-Tetramethyl-ethane-1,2-diamine (TEMED)

Nonidet P40 (NP-40)

Opti-MEM I

PageRuler Prestained Protein Ladder

Paraformaldehyde

Pen Strep (Penicillin Streptomycin)

Phenylmethanesulfonylfluoride (PMSF)
Merck (Darmstadt, Germany)

Sigma (Deisenhofen, Germany)

Merck (Darmstadt, Germany)

Sigma (Deisenhofen, Germany)

VWR International (Darmstadt, Germany)

Sigma (Deisenhofen, Germany)

Sigma (Deisenhofen, Germany)

Gibco (Paisley, UK)

Invitrogen (Carlsbad, USA)

Invitrogen (Carlsbad, USA)

Invitrogen (Carlsbad, USA)

Sigma (Deisenhofen, Germany)

Sigma (Deisenhofen, Germany)

Merck (Darmstadt, Germany)

BioMol (Hamburg, Germany)

Sigma (Deisenhofen, Germany)

Sigma (Deisenhofen, Germany)

Roche (Basel, Switzerland)

Gibco (Paisley, UK)

Thermo Scientific (Colorado, USA)

Merck (Darmstadt, Germany)

Gibco (Paisley, UK)

Serva (Heidelberg, Germany) 
Phosphate Buffered Saline (PBS) pH 7.2

Ponceau S

Potassium Acetate

Potassium Chloride

Potassium Dihydrogen Phosphate

Potassium Phosphate

Rubidium Chloride

Skim Milk Powder

Sodium Chloride

Sodium Deoxycholate

Sodium Dihydrogen Phosphate-monohydrate

Sodium Hydroxide

Sodiumdodecylsulfate (SDS)

Sodium-Ethylenediaminetetraacetic acid (EDTA)

Sybr Safe DNA gel stain

Trans- 1,2- cyclo- hexanediaminetetraacetate (CDTA)

Tris (Trizma base)

Triton X-100

TrypLE ${ }^{\mathrm{TM}}$ Express

Tween 20

$\beta$-Mercaptoethanol
Gibco (Paisley, UK)

Sigma (Deisenhofen, Germany)

Sigma (Deisenhofen, Germany)

Merck (Darmstadt, Germany)

Carl Roth GmbH (Karlsruhe, Germany)

Carl Roth GmbH (Karlsruhe, Germany)

Sigma (Deisenhofen, Germany)

Gabler \& Saliter (Oberguenzburg, Germany)

Merck (Darmstadt, Germany)

Sigma (Deisenhofen, Germany)

Merck (Darmstadt, Germany)

Merck (Darmstadt, Germany)

Serva (Heidelberg, Germany)

Merck (Darmstadt, Germany)

Invitrogen (Carlsbad, USA)

Sigma (Deisenhofen, Germany)

Sigma (Deisenhofen, Germany)

Sigma (Deisenhofen, Germany)

Gibco (Paisley, UK)

Sigma (Deisenhofen, Germany)

Merck (Darmstadt, Germany) 


\section{IV.1.2. Materials and Instruments}

$\mu$-Slide 8 well for Live Cell Analysis

$15 \mathrm{ml}$ Polypropylene Conical Tube

$50 \mathrm{ml}$ Polypropylene Conical Tube

5415C and 5417R Centrifuges

Accumet Basic pH Meter

AG285 and PB602 Balances

Avanti J-25 Centrifuge with Rotors JLA 10.500 and JA 25.50

$\mathrm{CO}_{2}$ Water Jacketed Incubator

Cryo-tubes

DNA Speedvac DNA110

DU 640 UV/VIS Spectrophotometer

Fluorescence Microscope Axiovert 200M

Gel Documentation System BioCapt

Gilson Pipetman 2, 10, 20, 100, 200 and $1000 \mu \mathrm{L}$

ImageReader LAS-3000

Innova 4430 Incubator

Light Microscope Diavert

Luminometer Lumat LB 9507

Millex SV Filter Units, pore size $0.22 \mu \mathrm{M}$

MilliQ Plus Deionization System

MiniProtean2 Electrophoresis Chamber
IBIDI (Martinsried, Germany)

Becton and Dickinson (New Jersey, USA)

Becton and Dickinson (New Jersey, USA)

Eppendorf (Hamburg, Germany)

Fisher Scientific (Schwerte, Germany)

Mettler Toledo (Gießen, Germany)

Beckman (Munich, Germany)

Forma Scientific (Waltham, USA)

Nunc (Roskilde, Denmark)

Savant (New York, USA)

Beckman (Munich, Germany)

Zeiss (Jena, Germany)

MWG BiotechAG (Göttingen, Germany)

Abimed (Langenfeld, Germany)

FUJI (Tokyo, Japan)

New Brunswick Scientific (Nürtingen, Germany)

Leitz (Wetzlar, Germany)

Berthold (Bad-Wildbad, Germany)

Millipore (Eschborn, Germany)

Millipore (Eschborn, Germany)

Bio-Rad (Munich, Germany) 
Nanodrop 1000 Spectrophotometer

Nitrocellulose Membrane, PROTRAN

PCR Tubes AB-0266

pH-Meter pH538

Poly-L-Lysine Round Coverslips, 12mm

Safe Imager 2.0 Blue-Light Transilluminator

Scepter ${ }^{\mathrm{TM}}$ Automated Cell Counter

Scepter ${ }^{\mathrm{TM}}$ Sensors - 60 $\mu \mathrm{M}$

Tank Blot System

TCS SP2 Confocal Laser Scanning Microscope

Thermocycler PCR T3

Thermomixer Comfort

Tissue Culture Dish 100 x 20 mm

Tissue Culture Plate, 96 well

Tissue Culture Plate, 48 well

Tissue Culture Plate, 12 well

Tissue Culture Plate, 24 well

Tissue Culture Plate, 6 well

Transformer for Electrophoresis PAC300

UltraVIEW Vox Spinning Disk Confocal Microscope

Weighing Balance CP3202P
Thermo Scientific (Colorado, USA)

Schleicher \& Schuell (Dassel, Germany)

Thermo Scientific (Colorado, USA)

WTW (Weilheim, Germany)

BD Biosciences (CA, USA)

Invitogen (Carlsbad, USA)

Millipore (MA, USA)

Millipore (MA, USA)

Bio-Rad (Munich, Germany)

Leica (Wetzlar, Germany)

Biometra (Göttingen, Germany)

Eppendorf (Hamburg, Germany)

Becton and Dickinson (New Jersey, USA)

Becton and Dickinson (New Jersey, USA)

Becton and Dickinson (New Jersey, USA)

Becton and Dickinson (New Jersey, USA)

Becton and Dickinson (New Jersey, USA)

Becton and Dickinson (New Jersey, USA)

Bio-Rad (Munich, Germany)

Perkin Elmer (MA, USA)

Sartorius (Göttingen, Germany) 


\section{IV.1.3. Enzymes}

DpnI

$P f u$ DNA Polymerase

Proteinase K

Restriction Enzymes

$\mathrm{T}_{4}$ DNA Ligase

\section{IV.1.4. Bacterial Strains}

DH5 $\alpha F^{\prime}$

SURE

\section{IV.1.5. Softwares}

Aida Image Analyzer 4.15.025

Adobe Photoshop CS5.1

Axiovision Rel 4.7 software

Chromas version 1.45

FIJI

Leica TCS Analysis

Sigma Plot 10.0

Volocity 6.0
New England Biolabs (Ipswich, USA)

Promega (Madison, USA)

Merck (Darmstadt, Germany)

New England Biolabs (Ipswich, USA)

New England Biolabs (Ipswich, USA)
$F^{\prime}$ lendA1 hsdR17 $\left(r_{k}{ }^{-}, m_{k}{ }^{+}\right)$glnV44 thi-1 recAl gyrA $\left(N A I^{r}\right.$ ) relA14 (lacIZYA-argF) U169 deoR [p80dlacd (lacZ)M15]

e14-(McrA-) $\quad \triangle($ mcrCB-hsdSMR-mrr $) 171$ endA1 supE44 thi-1 gyrA96 relA1 lac recB recJ sbcC uтиC::Tn5 $\left(\operatorname{Kan}^{R}\right)$ uvrC[F' $\operatorname{proAB} \operatorname{lacl}^{q} Z \Delta M 15$ Tn10 $\left(\right.$ Tet $\left.\left.^{\mathrm{r}}\right)\right]$ 


\section{IV.1.6. Kits Used}

\begin{tabular}{|l|c|c|}
\hline \multicolumn{1}{|c|}{ Name } & Cat. No. & Company \\
\hline Dual Glo Luciferase Assay System & E2920 & Promega (Madison, USA) \\
\hline iScript Select cDNA Synthesis Kit & $170-8896$ & Bio-Rad (Munich, Germany) \\
\hline Gel and PCR Clean Up System & A1460 & Promega (Madison, USA) \\
\hline Luciferase Assay System & E1510 & Promega (Madison, USA) \\
\hline Minipreps Wizard Plus & A9282 & Promega (Madison, USA) \\
\hline Plasmid Midiprep System & A2495 & Promega (Madison, USA) \\
\hline Steady Glo Luciferase Assay System & E2510 & Promega (Madison, USA) \\
\hline $\begin{array}{l}\text { TnT T7 Quick coupled } \\
\text { Transcription/Translation System }\end{array}$ & L1170 & Promega (Madison, USA) \\
\hline
\end{tabular}

\section{IV.1.7. Antibodies}

\begin{tabular}{|l|c|l|}
\hline \multicolumn{1}{|c|}{ Name } & Cat. No. & \multicolumn{1}{c|}{ Company } \\
\hline Anti-Luciferase pAb & G7451 & Promega (Madison, USA) \\
\hline Anti-GFP & 11814460001 & Roche (Basel, Switzerland) \\
\hline Anti-GAPDH & MAB374 & Millipore (MA, USA) \\
\hline Anti-c-Myc (9E10) & sc- 40 & Santa Cruz Biotechnology Inc. (CA, USA) \\
\hline Anti-Renilla Luciferase & MAB4410 & Millipore (MA, USA) \\
\hline Anti-Hsc70 (1B5) & sc-59560 & Santa Cruz Biotechnology Inc. (CA, USA) \\
\hline Anti-Hsp40 & 4868 & Cell Signaling Technology (MA, USA) \\
\hline Anti-20S Proteasome & ab22673 & Abcam (Cambridge, UK) \\
\hline Anti-Ubiquitin (FK2) & $04-263$ & Millipore (MA, USA) \\
\hline $\begin{array}{l}\text { Anti-Mouse IgG (whole molecule) } \\
\text { Peroxidase Conjugate }\end{array}$ & A4416 & Sigma (Deisenhofen, Germany) \\
\hline $\begin{array}{l}\text { Anti-Rabbit IgG (whole molecule) } \\
\text { Peroxidase Conjugate }\end{array}$ & A9037 & Sigma (Deisenhofen, Germany) \\
\hline $\begin{array}{l}\text { Anti-Goat IgG (whole molecule) } \\
\text { Peroxidase Conjugate }\end{array}$ & A5420 & Sigma (Deisenhofen, Germany) \\
\hline Cy3 labeled Goat anti-mouse IgG & $115-165-062$ & Jackson ImmunoResearch (Suffolk, UK) \\
\hline Cy3 labeled Goat anti-rabbit IgG & $111-165-045$ & Jackson ImmunoResearch (Suffolk, UK) \\
\hline
\end{tabular}




\section{IV.2. Media and Buffers}

\section{IV.2.1. Media}

\section{LB Medium}

$10 \mathrm{~g} / \mathrm{l}$ Bacto tryptone, $5 \mathrm{~g} / \mathrm{l}$ Bacto yeast extract, $10 \mathrm{~g} / \mathrm{l} \mathrm{NaCl}$, $\mathrm{pH}$ adjusted to 7.0 with $\mathrm{NaOH}$.

\section{LB Agar}

$16 \mathrm{~g} / \mathrm{l} \mathrm{Bacto}$ agar dissolved in LB medium.

\section{NGM Agar}

$0.3 \%$ (w/v) $\mathrm{NaCl}, 0.25 \%$ (w/v) peptone, $25 \mathrm{mM} \mathrm{KPO}_{4}, 1 \mathrm{mM} \mathrm{CaCl}_{2}, 1 \mathrm{mM} \mathrm{MgSO}$, $5 \mathrm{mg}$ cholesterol (dissolved in ethanol) and 1.7\% (w/v) agar.

\section{IV.2.2. Buffers}

\section{X SDS Sample Buffer (Laemmli Buffer)}

$240 \mathrm{mM}$ Tris (pH 6.8), 8\% (w/v) SDS, 40\% (v/v) glycerol, 1.4 M $\beta$-Mercaptoethanol, $0.02 \%$ (w/v) bromphenol blue

\section{PBS (Phosphate buffered saline)}

$137 \mathrm{mM} \mathrm{NaCl}, 2.68 \mathrm{mM} \mathrm{KCl}, 10.1 \mathrm{mM} \mathrm{Na}_{2} \mathrm{HPO}_{4}, 1.76 \mathrm{mM} \mathrm{NaH}_{2} \mathrm{PO}_{4}, \mathrm{pH}$ adjusted to 7.4 with $\mathrm{HCl}$

\section{SDS-PAGE Running Buffer}

$50 \mathrm{mM}$ Tris- $\mathrm{HCl} \mathrm{pH}$ 8.3, $380 \mathrm{mM}$ glycine, $0.1 \%$ (w/v) SDS

1x TAE Agarose Gel Electrophoresis Buffer

$242 \mathrm{~g} / \mathrm{l}$ Tris base, $57.1 \mathrm{ml} / \mathrm{l}$ acetic acid, $50 \mathrm{mM}$ EDTA

\section{TBS (Tris buffered saline)}

25 mM Tris- $\mathrm{HCl}, \mathrm{pH} 7.2,150 \mathrm{mM} \mathrm{NaCl}$

TBST $($ TBS + Tween 20)

$0.1 \%(\mathrm{v} / \mathrm{v})$ Tween 20 in TBS 


\section{Transfer Buffer for Western Blotting}

$25 \mathrm{mM}$ Tris, $192 \mathrm{mM}$ glycine, 20\% (v/v) methanol, $\mathrm{pH} 8.4$

\section{Cell Lysis Buffer}

$50 \mathrm{mM}$ Tris (pH 7.8), $150 \mathrm{mM} \mathrm{NaCl}, 0.25 \%$ (w/v) sodium deoxycholate, $1 \%$ (v/v) NP-40, $1 \mathrm{mM}$ EDTA and 1 tablet protease inhibitor cocktail per $10 \mathrm{ml}$.

\section{SDT Buffer}

4\% (w/v) SDS, 100 mM Tris-HCl (pH 7.6) and 0.1 M DTT

\section{Stop Buffer}

25 mM Tris-phosphate (pH 7.8), 2 mM CDTA, $1 \mathrm{mg} / \mathrm{ml} \mathrm{BSA}$ and $2 \mathrm{mM}$ cycloheximide

\section{IV.3. Molecular Cloning Methods}

All routine molecular biology methods such as polymerase chain reaction (PCR), agarose gel electrophoresis, DNA quantification, competent cell preparation and transformation of bacterial cells etc. were performed according to "Molecular cloning" (Sambrook, 2001) unless otherwise stated. Plasmid DNA was isolated from DH5 $\alpha$ or SURE (for isolation of Htt-20QmCherry and Htt-97Q-mCherry constructs) cells using Plasmid Midiprep System (Promega) according to manufacturer's protocol. Only high quality DNA (with $\mathrm{A}_{260 / 280}>1.85$ and $\mathrm{A}_{260 / 230}>$ 2.10) was used for in vitro experiments in rabbit reticulocyte lysate and for transfection of mammalian cells. Primers used for PCR amplification were purchased from Metabion (Martinsried, Germany). Routine plasmid preparation for cloning was done using Minipreps Wizard Plus kit (Promega). PCR clean up and gel purification of DNA for cloning purpose was done using Gel and PCR Clean up System (Promega). DNA sequencing was performed by the sequencing facility at MPI core facility (Martinsried, Germany). 


\section{IV.3.1. Preparation and Transformation of Chemically Competent E. coli Cells}

For preparation of chemically competent $E$. coli cells, a single colony of DH5 $\alpha$ or SURE strain was grown in LB overnight with continuous shaking at $37^{\circ} \mathrm{C}$. Next day, $5 \mathrm{~mL}$ of bacterial culture was inoculated in $500 \mathrm{~mL}$ fresh $\mathrm{LB}$ medium and grown to an optical density (O.D. 600 ) of 0.5 at $37^{\circ} \mathrm{C}$. The cells were then centrifuged in sterile tubes at $5000 \mathrm{~g}$ for $10 \mathrm{~min}$ at $4{ }^{\circ} \mathrm{C}$. The cell pellet was resuspended in $125 \mathrm{ml}$ ice-cold transformation buffer I (30 mM potassium acetate, 50 $\mathrm{mM}$ manganese chloride, $100 \mathrm{mM}$ rubidium chloride, $10 \mathrm{mM}$ calcium chloride and $15 \%$ glycerol) and incubated on ice for $20 \mathrm{~min}$. After incubation, the cells were again centrifuged at $5000 \mathrm{~g}$ for $10 \mathrm{~min}$ at $4^{\circ} \mathrm{C}$ and the pellet was gently resuspended in $20 \mathrm{ml}$ ice-cold transformation buffer II (10 mM MOPS, $75 \mathrm{mM}$ calcium chloride, $10 \mathrm{mM}$ rubidium chloride and $15 \%$ glycerol). Finally, $50 \mu \mathrm{l}$ aliquots were frozen in liquid nitrogen and stored at $-80^{\circ} \mathrm{C}$.

For transformation, $50 \mu \mathrm{l}$ chemically competent E. coli cells were mixed with 50-100 ng plasmid DNA or $20 \mu \mathrm{l}$ ligation mixture and incubated on ice for $30 \mathrm{~min}$. The cells were heat shocked at $42^{\circ} \mathrm{C}$ for $50 \mathrm{sec}$ and tubes were immediately placed on ice for $2 \mathrm{~min} .1 \mathrm{ml}$ of $\mathrm{LB}$ medium was subsequently added and the cells were shaken at $37^{\circ} \mathrm{C}$ for $1 \mathrm{~h}$. The cell suspension was then plated on LB agar plates containing suitable antibiotic(s) and incubated at $37^{\circ} \mathrm{C}$ until colonies had appeared.

\section{IV.3.2. PCR Amplification}

PCR mediated amplification of plasmid DNA or linear DNA was performed by setting a $25 \mu \mathrm{l}$ PCR reaction containing the following components

\begin{tabular}{|l|l|}
\hline DNA Template & 20 ng DNA \\
\hline Primers & 20 pmole each \\
\hline dNTPs & $200 \mu \mathrm{M}$ each \\
\hline Pfu Polymerase & 2.5 units \\
\hline Polymerase reaction buffer (with $\left.\mathrm{Mg}^{2+}\right)$ & $1 \mathrm{X}$ \\
\hline Additives & $3-6 \%$ DMSO if GC content $>50 \%$ \\
\hline
\end{tabular}


PCR Cycling conditions:

\begin{tabular}{|l|c|c|}
\hline Reaction Step & Temp & Time \\
\hline Initial Denaturation & $95^{\circ} \mathrm{C}$ & $3 \mathrm{~min}$ \\
\hline Cycle Denaturation & $95^{\circ} \mathrm{C}$ & $30-60 \mathrm{sec}$ \\
\hline Annealing (dependent on Tm of primers) & $\sim 55^{\circ} \mathrm{C}$ & $30-60 \mathrm{sec}$ \\
\hline Extension (duration dependent on template length) & $72^{\circ} \mathrm{C}$ & $500 \mathrm{bp} / \mathrm{min}$ \\
\hline Final Extension & $72^{\circ} \mathrm{C}$ & $15 \mathrm{~min}$ \\
\hline
\end{tabular}

\section{IV.3.3. Site-Directed Mutagenesis PCR}

Site-directed mutagenesis was done to introduce mutations at specific positions in Firefly luciferase (Fluc) to generate mutants. Briefly, the plasmid was PCR amplified using Pfu polymerase and primers as described before. The PCR product was digested with $1 \mu 1 \mathrm{Dpn}$ I (New England Biolabs) in appropriate buffer for $2 \mathrm{~h}$ at $37^{\circ} \mathrm{C}$ to cleave the parental (methylated) DNA. The PCR product was then used to transform chemically competent E. coli cells and plated on suitable LB agar plates. The plates were incubated overnight at $37^{\circ} \mathrm{C}$ until colonies had appeared. The colonies were inoculated into fresh LB medium with suitable antibiotic and plasmid was isolated using Minipreps Wizard Plus Kit (Promega). The constructs were routinely sequenced to confirm the mutations.

\section{IV.3.4. DNA Restriction and Ligation}

DNA digestion was performed using restriction endonuclease enzymes according to the manufacturer's instructions (New England Biolabs). A $20 \mu$ l digestion reaction was set containing $2 \mu \mathrm{g}$ plasmid DNA or PCR product and 1-2 $\mu 1$ of each restriction enzyme in the appropriate reaction buffer. The digested DNA was separated from the uncut DNA by agarose gel electrophoresis. The digested DNA was purified from the agarose gel using Gel and PCR Clean up System (Promega). DNA concentrations were measured by UV absorption spectroscopy at $\lambda=260 \mathrm{~nm}$.

For ligation reaction, $20 \mu 1$ reaction was set containing 100-200 ng digested vector DNA, 100-200 ng insert DNA in molar ratio of 1:3 (vector:insert) and 100 units of $\mathrm{T}_{4}$ DNA ligase in 
ligase buffer. The reaction mixture was incubated at $16^{\circ} \mathrm{C}$ for overnight. The ligation product was transformed into competent $E$. coli cells as described.

\section{IV.3.5. Generation of Expression Constructs}

Following primers were used for the cloning of various constructs used in this study. The underlined residues indicate restriction endonuclease sites introduced for cloning.

\begin{tabular}{|c|c|}
\hline Primer & Sequence \\
\hline Fluc FOR & 5'- CCGCTCGAGCATGGAAGACGCCAAAAAC - 3' \\
\hline Fluc REV & 5'- TCCCCCCGGGTTACAATTTGGACTTTCCGC - 3' \\
\hline Rluc FOR & 5'- ACGCCTCGAGATGGCTTCCAAGGTGTACGA - 3' \\
\hline Rluc REV & 5'- ACGCGGCCGGCCTTACTGCTCGTTCTTCAGCACG - 3' \\
\hline NPM1 FOR & 5'- CGGGATCCCGATGGAAGATTCGATGGACATGGAC - 3' \\
\hline NPM1 REV & 5'- CGGAATTCCGGTGGAGGTGAAAGAGACTTCCTCCACTGCC - 3' \\
\hline
\end{tabular}

\section{Cloning of Firefly Luciferase in Mammalian Expression Vector}

The Firefly luciferase (Fluc) gene from plasmid pGL3 basic (Promega, Madison, USA) encoding residues 1-550 of wild-type Fluc (with the C-terminal peroxisomal targeting signal Serlys-Leu replaced by Ile-Ala-Val and containing two point mutations, Asn50Asp and Asn119Gly, to improve efficiency of the enzyme) was PCR amplified using Fluc FOR and REV primers. The PCR product was digested with XhoI and XmaI restriction endonucleases and sub-cloned into the pCI-neo vector (Promega) to form pCIneoFluc. pCIneoFluc constructs were used for in vitro translation in rabbit reticulocyte lysate (RRL) and for expression in mammalian cell culture.

\section{Generation of Fluc mutants}

To generate Fluc mutants, site-specific mutations were done using site-directed mutagenesis primers. Following primers were used to introduce mutations in Fluc gene in pCIneoFluc plasmid in order to generate Fluc mutants. 


\begin{tabular}{|c|c|}
\hline Primer & Sequence \\
\hline K135Q FOR & 5' - CCAAAAAGGGGTTGCAACAAAATTTTGAACGTGCAA - 3' \\
\hline K135Q REV & 5'- TTGCACGTTCAAAATTTGTTTGCAACCCCTTTTTGG - 3' \\
\hline K135M FOR & 5' - CCAAAAAGGGGTTGCAAATGATTTTGAACGTGCAA - 3' \\
\hline K135M REV & 5'- GCGGCCGGCCAATAAGCTTTTGTTCTTTCTGC - 3' \\
\hline R188Q FOR & 5' - CGATTTTGTGCCAGAGTCCTTCGATCAGGACAAGACAATTGC - 3' \\
\hline R188Q REV & 5' - GCAATTGTCTTGTCCTGATCGAAGGACTCTGGCACAAAATCG - 3' \\
\hline R188K FOR & 5' - CGATTTTGTGCCAGAGTCCTTCGATAAAGACAAGACAATTGC - 3' \\
\hline R188K REV & 5' - GCAATTGTCTTGTCTTTATCGAAGGACTCTGGCACAAAATCG - 3' \\
\hline R261Q FOR & 5'- CGGATATTTGATATGTGGATTTCÁAGTCGTCTTAATG - 3' \\
\hline R261Q REV & 5'- CATTAAGACGACTTGAAATCCACATATCAAATATCCG - 3' \\
\hline R261K FOR & 5'- CGGATATTTGATATGTGGATTTAAAGTCGTCTTAATG - 3' \\
\hline R261K REV & 5'- CATTAAGACGACTTTAAATCCACATATCAAATATCCG - 3' \\
\hline $\begin{array}{l}\text { Fluc Stop } \\
\text { removal FOR }\end{array}$ & 5' - GGAAAGATCGCCGTGAAACCCGGGATCCACCGGTC - 3' \\
\hline $\begin{array}{l}\text { Fluc Stop } \\
\text { removal REV }\end{array}$ & 5' - GACCGGTGGATCCCGGGTTTCACGGCGATCTTTCC - 3' \\
\hline
\end{tabular}

To generate EGFP-tagged constructs, the stop codon of the luciferase gene in the pCIneoFluc was changed to AAA, encoding lysine. EGFP from pEGFP-N2 vector (Clontech, CA, USA) was inserted at the 3' end of Fluc in pCIneoFluc plasmid using XmaI and NotI restriction sites to generate pCIneoFluc-EGFP. To generate pCIneoFlucSM-EGFP, site-directed mutagenesis using primers R188Q FOR and REV and pCIneoFluc-EGFP as DNA template was performed. To generate pCIneoFlucDM-EGFP, another round of site-directed mutagenesis using primers R261Q FOR and R261Q and pCIneoFlucSM-EGFP as DNA template was performed.

EGFP-tagged luciferase variants were cloned for $C$. elegans in a similar manner in the pPD30-38 vector (under the unc-54 promoter) for muscle-specific expression and in pDP\#SU006 (under the F25B3.3 promoter) for neuron-specific expression. The Fluc-EGFP constructs for expression in $C$. elegans were prepared by Dr. Prasad Kasturi. 


\section{Generation of Huntingtin Constructs}

Huntingtin constructs were prepared by cloning the exon1 fragment of huntingtin gene (Htt) containing sequence encoding the polyglutamine region (20Q or 97Q) (Behrends et al., 2006) N-terminally fused to mCherry in the pcDNA3.1(+) mammalian expression vector (Invitrogen). The restriction endonucleases used were BamHI and XhoI. For plasmid propagation, chemically competent SURE cells were used. The length of the polyQ stretch in all constructs was verified by DNA sequencing.

\section{Generation of HSPA1A-Rluc-myc construct}

The construct HSPA1A-Rluc-myc expresses C-terminally Myc-tagged Renilla luciferase (Rluc) under control of the HSF1 dependent HSPA1A promoter. To generate this construct, the Rluc gene was PCR amplified from the phRL-TK vector (Promega) using primers Rluc FOR and REV. The PCR product was digested with XhoI and FseI restriction endonucleases and subcloned into plasmid pUB/Bsd HSP70-Luc (Fluc gene under control of the HSPA1A promoter, gift from Prof. H. Wagner, Technical University of Munich) by replacing the Fluc gene. A myc tag was introduced at the 3 ' end of the Rluc gene by four rounds of sequential site-directed mutagenesis using the following primers

\begin{tabular}{|l|l|}
\hline Primer & Sequence \\
\hline Myc1 FOR & 5'- GAGCAGAAAGAACAAAGGCCGGCCGC - 3' \\
\hline Myc1 REV & 5'- GCGGCCGGCCTTTGTTCTTTCTGCTC - 3' \\
\hline Myc2 FOR & 5'- GCAGAAAGAACAAAAGCTTATTGGCCGGCCGC - 3' \\
\hline Myc2 REV & 5'- GCGGCCGGCCAATAAGCTTTTGTTCTTTCTGC - 3' \\
\hline Myc3 FOR & 5'- AACAAAAGCTTATTTCTGAAGAGGCCGGCCGC - 3' \\
\hline Myc3 REV & 5'- GCGGCCGGCCTCTTCAGAAATAAGCTTTTGTT - 3' \\
\hline Myc4 FOR & 5'- TTATTTCTGAAGAAGACTTGGGCCGGCCGC - 3' \\
\hline Myc4 REV & 5'- GCGGCCGGCCCAAGTCTTCTTCAGAAATAA - 3' \\
\hline
\end{tabular}

The residues that were added in each subsequent step to introduce myc tag are underlined. 


\section{Generation of NLS- and NES-Fluc-EGFP Constructs}

To generate constructs for NLS- and NES-Fluc-EGFP variants, NLS (Fischer-Fantuzzi and Vesco, 1988) and NES (la Cour et al., 2004) sequences were added 3' end of CMV promoter in pcDNA3.1 myc his A plasmid (Invitrogen) by sequential site-directed mutagenesis. The FlucEGFP gene was PCR amplified and subcloned at the 3' end of NLS or NES sequence in pCDNA3.1 myc his A vector using restriction enzymes KpnI and XbaI. NLS- and NESFlucDM-EGFP constructs were generated by site-directed mutagenesis of NLS- and NES-FlucEGFP constructs, respectively. All constructs were verified by DNA sequencing. The constructs expressing NLS- and NES-Fluc-EGFP variants were generated by Andreas Wörner.

\section{Generation of NPM1-mCherry Construct}

To generate NPM1-mCherry construct, NPM1 or B23 gene (Cluster ID- Hs.557550 and Library Number- NIH_MGC_68) was PCR amplified from human genome library collection (MPI core facility, Martinsried, Germany) using primers NPM1 FOR and REV. The purified PCR product was digested with restriction endonucleases EcoRI and BamHI and subcloned upstream of mCherry by replacing Htt-20Q in Htt-20Q-mCherry plasmid. The sequence of resultant construct was verified by DNA sequencing.

\section{IV.3.5.1. Constructs}

Following constructs were used in this study

\begin{tabular}{|l|l|c|c|}
\hline \multicolumn{1}{|c|}{ Name } & Plasmid backbone & \multicolumn{2}{c|}{ Antibiotic resistance } \\
\hline & & 1 & 2 \\
\hline wild type (Fluc) & pCI-neo & Ampicillin & Neomycin \\
\hline K135Q & pCI-neo & Ampicillin & Neomycin \\
\hline K135M & pCI-neo & Ampicillin & Neomycin \\
\hline R188Q (FlucSM) & pCI-neo & Ampicillin & Neomycin \\
\hline R188K & pCI-neo & Ampicillin & Neomycin \\
\hline R261Q & pCI-neo & Ampicillin & Neomycin \\
\hline R261K & pCI-neo & Ampicillin & Neomycin \\
\hline K135Q+R188Q & pCI-neo & Ampicillin & Neomycin \\
\hline
\end{tabular}




\begin{tabular}{|c|c|c|c|}
\hline K135Q+R188K & pCI-neo & Ampicillin & Neomycin \\
\hline K135Q+R261Q & pCI-neo & Ampicillin & Neomycin \\
\hline K135Q+R261K & pCI-neo & Ampicillin & Neomycin \\
\hline K135M+R188Q & pCI-neo & Ampicillin & Neomycin \\
\hline $\mathrm{K} 135 \mathrm{M}+\mathrm{R} 188 \mathrm{~K}$ & pCI-neo & Ampicillin & Neomycin \\
\hline $\mathrm{K} 135 \mathrm{M}+\mathrm{R} 261 \mathrm{Q}$ & pCI-neo & Ampicillin & Neomycin \\
\hline $\mathrm{K} 135 \mathrm{M}+\mathrm{R} 261 \mathrm{~K}$ & pCI-neo & Ampicillin & Neomycin \\
\hline R188Q+R261Q (FlucDM) & pCI-neo & Ampicillin & Neomycin \\
\hline R188Q+R261K & pCI-neo & Ampicillin & Neomycin \\
\hline $\mathrm{R} 188 \mathrm{~K}+\mathrm{R} 261 \mathrm{Q}$ & pCI-neo & Ampicillin & Neomycin \\
\hline $\mathrm{R} 188 \mathrm{~K}+\mathrm{R} 261 \mathrm{~K}$ & pCI-neo & Ampicillin & Neomycin \\
\hline Fluc-EGFP & pCI-neo & Ampicillin & Neomycin \\
\hline FlucSM-EGFP & pCI-neo & Ampicillin & Neomycin \\
\hline FlucDM-EGFP & pCI-neo & Ampicillin & Neomycin \\
\hline HSPA1A Rluc myc & $\mathrm{pUB} / \mathrm{Bsd}$ & Ampicillin & Blasticidin \\
\hline Htt-97Q-mCherry & pcDNA3.1(+) & Ampicillin & Hygromycin B \\
\hline Htt-20Q-mCherry & pcDNA3.1(+) & Ampicillin & Hygromycin B \\
\hline NLS-Fluc-EGFP & pcDNA3.1 myc his A & Ampicillin & Neomycin \\
\hline NLS-FlucDM-EGFP & pcDNA3.1 myc his A & Ampicillin & Neomycin \\
\hline NES-Fluc-EGFP & pcDNA3.1 myc his A & Ampicillin & Neomycin \\
\hline NES-FlucDM-EGFP & pcDNA3.1 myc his A & Ampicillin & Neomycin \\
\hline NPM1-mCherry & pcDNA3.1(+) & Ampicillin & Hygromycin B \\
\hline unc-54 Fluc-EGFP & pPD30-38 & Ampicillin & ------ \\
\hline unc-54 FlucSM-EGFP & pPD30-38 & Ampicillin & ------ \\
\hline unc-54 FlucDM-EGFP & pPD30-38 & Ampicillin & ------ \\
\hline F25B3.3 Fluc-EGFP & pDP\#SU006 & Ampicillin & ------ \\
\hline F25B3.3 FlucSM-EGFP & pDP\#SU006 & Ampicillin & ------ \\
\hline F25B3.3 FlucDM-EGFP & pDP\#SU006 & Ampicillin & ------ \\
\hline
\end{tabular}




\section{IV.4. Protein Analytical Methods}

\section{IV.4.1. Sodium Dodecyl Sulfate-Polyacrylamide Gel Electrophoresis (SDS-PAGE)}

SDS-Polyacrylamide gels were prepared as follows

\begin{tabular}{|l|c|c|c|c|}
\hline \multicolumn{1}{|c|}{ Chemicals } & \multicolumn{2}{c|}{ 12\% Separating Gel } & \multicolumn{2}{c|}{ 4\% Stacking Gel } \\
\hline & $\mathbf{1 0} \mathbf{~ m l}$ & $\mathbf{1 5} \mathbf{~ m l}$ & $\mathbf{4 ~ m l}$ & $\mathbf{8 ~ m l}$ \\
\hline Distilled $\mathrm{H}_{2} \mathrm{O}$ & $3.3 \mathrm{ml}$ & $4.9 \mathrm{ml}$ & $2.7 \mathrm{ml}$ & $5.5 \mathrm{ml}$ \\
\hline $30 \%$ Acrylamide mix & $4 \mathrm{ml}$ & $6 \mathrm{ml}$ & $670 \mu \mathrm{l}$ & $1.3 \mathrm{ml}$ \\
\hline $1.5 \mathrm{M}$ Tris $(\mathrm{pH}-8.8)$ & $2.5 \mathrm{ml}$ & $3.8 \mathrm{ml}$ & ----- & ----- \\
\hline $1 \mathrm{M}$ Tris $(\mathrm{pH}-6.8)$ & ----- & ----- & $500 \mu \mathrm{l}$ & $1 \mathrm{ml}$ \\
\hline $10 \%$ SDS & $100 \mu \mathrm{l}$ & $150 \mu \mathrm{l}$ & $40 \mu \mathrm{l}$ & $80 \mu \mathrm{l}$ \\
\hline $10 \%$ APS & $100 \mu \mathrm{l}$ & $150 \mu \mathrm{l}$ & $40 \mu \mathrm{l}$ & $80 \mu \mathrm{l}$ \\
\hline TEMED & $4 \mu \mathrm{l}$ & $6 \mu \mathrm{l}$ & $4 \mu \mathrm{l}$ & $8 \mu \mathrm{l}$ \\
\hline
\end{tabular}

SDS-PAGE was performed using a discontinuous buffer system (Laemmli, 1970) in BioRad Mini-Protean2 electrophoresis chambers employing a constant current of $30 \mathrm{~mA}$ in SDSPAGE running buffer. Protein samples for SDS-PAGE were prepared by mixing the samples (cell lysate or soluble fraction) with 2X Laemmli loading buffer (Laemmli, 1970). Prior to loading, samples were boiled at $95^{\circ} \mathrm{C}$ for $5 \mathrm{~min}$.

\section{IV.4.2. Western Blotting}

Proteins were resolved by SDS-PAGE and gels were transferred onto nitrocellulose membranes in a Mini Trans-Blot Electrophoretic Transfer Cell (Bio-Rad) in transfer buffer (25 $\mathrm{mM}$ Tris, $192 \mathrm{mM}$ glycine, 20\% (v/v) methanol, $\mathrm{pH} 8.4$ ) at constant current of $250 \mathrm{~mA}$ for 1 gel (300 mA for 2 gels) for $90 \mathrm{~min}$. Following transfer, the membranes were blocked in 5\% skim milk powder in TBST solution for $1 \mathrm{~h}$. The membranes were then incubated with primary antibody (diluted in blocking solution) for $1 \mathrm{~h}$ at room temperature or overnight at $4^{\circ} \mathrm{C}$. The membranes were extensively washed with TBST solution for 3-4 times (each washing of 5 min), followed by incubation with HRP-conjugated (horseradish peroxidase) secondary antibody in 
TBST for $3 \mathrm{~h}$ at room temperature. After extensive washing with TBST solution for 3-4 times, protein bands were detected by incubating the membranes with ECL detection reagent and using Luminiscent ImageReader LAS-3000 system (FUJI).

\section{IV.4.3. Protein Quantification by Bradford's Assay}

Soluble fractions of proteins were quantified in a calorimetric assay based on the method developed by Bradford (Bradford, 1976) using Bio-Rad protein assay reagent. As a reference, a calibration curve of BSA was used. Absorbance values were recorded at $595 \mathrm{~nm}$ using DU 640 UV/VIS Spectrophotometer (Beckman).

\section{IV.5. In Vitro Characterization of Fluc Mutants}

\section{IV.5.1. Thermal Denaturation of Fluc Variants in RRL}

Luciferase (Fluc) mutants were transcribed and translated in rabbit reticulocyte lysate (RRL) using the Promega TnT T7 quick coupled transcription and translation kit as recommended by the manufacturer. Unless otherwise indicated, pCI-neo constructs of Fluc variants were incubated in $\mathrm{RRL}$ at $30^{\circ} \mathrm{C}$ for 90 minutes. To analyze the functional stability of the mutant proteins, translation reaction was stopped by three-fold dilution of samples into ice-cold stop buffer (25 mM Tris-phosphate $\mathrm{pH}$ 7.8, $2 \mathrm{mM}$ trans-1,2-cyclo- hexanediaminetetraacetate (CDTA) and $1 \mathrm{mg} \mathrm{ml}^{-1} \mathrm{BSA}$ ) containing $2 \mathrm{mM}$ cycloheximide. The reaction mixtures were incubated at various temperatures, and luciferase activity was determined using Luminometer Lumat LB 9507 (Berthold) at the indicated times by mixing $2 \mu 1$ sample in $30 \mu 1$ Luciferase Assay System (Promega). The measuring time was set to $0.1 \mathrm{sec}$ in the instrument.

\section{IV.5.2. Measurement of Fluc Specific Activity in RRL}

For specific activity measurements (luciferase activity normalized to the luciferase protein content in the sample), Fluc mutant proteins were transcribed and translated in RRL at $30^{\circ} \mathrm{C}$ for 90 minutes. Translation reaction was stopped by addition of stop buffer containing $\mathrm{CHX}$, as described above. Aliquots at $30^{\circ} \mathrm{C}$ were withdrawn at $0 \mathrm{~min}$ and $120 \mathrm{~min}$ and luciferase activity was measured. To measure Fluc amount, $2 \mu 1$ RRL sample was mixed in $20 \mu 1 \mathrm{Laemmli}$ loading buffer and ran on SDS-PAGE. Fluc amount was analyzed by immunoblotting with 
luciferase antibody (Promega G7451, polyclonal, which recognizes wild-type and mutant luciferase proteins with similar efficiency).

\section{IV.5.3. Assessment of Co-translational Folding Efficiency of Fluc Variants in RRL}

To check for the efficiency of co-translational folding of Fluc mutants, Fluc DNA was added to RRL at $30^{\circ} \mathrm{C}$ and $1 \mu \mathrm{l}$ of sample was withdrawn at different times and mixed with $30 \mu \mathrm{l}$ Luciferase Assay System (Promega) to measure the luciferase activity.

\section{IV.5.4. Limited Proteolysis by Proteinase K}

To assess the structural flexibility, Fluc variants were transcribed and translated in RRL at $30^{\circ} \mathrm{C}$ for $90 \mathrm{~min}$ followed by three-fold dilution in stop buffer containing $2 \mathrm{mM}$ cycloheximide. The reaction mixture in each case was distributed into 2 aliquots for determining luciferase activity at $20^{\circ} \mathrm{C}$ in the presence and absence of proteinase $\mathrm{K}\left(1 \mu \mathrm{g} \mathrm{ml}^{-1}\right)$ (Merck) at different time points. Luciferase activity was measured as described before. The action of proteinase K was inhibited by addition of $5 \mathrm{mM}$ PMSF. Samples for SDS-PAGE were prepared by mixing $2 \mu \mathrm{l}$ diluted RRL in $20 \mu \mathrm{l}$ Laemmli loading buffer and boiling them for 5 min at $95^{\circ} \mathrm{C}$. The pattern of proteinase $\mathrm{K}$ digestion was visualized by immunoblotting with Fluc antibody.

\section{IV.5.5. Guanidinium Chloride Denaturation}

Fluc variants were synthesized in RRL as described above. Translation was stopped by addition of stop buffer containing $2 \mathrm{mM}$ cycloheximide. The proteins were denatured by ten-fold dilution of reaction mixture in ice cold Buffer B (20 mM HEPES- KOH (pH 7.4), 100 mM $\mathrm{KAcO}, 5 \mathrm{mM} \mathrm{Mg}(\mathrm{AcO})_{2}, 0.5 \mathrm{mM}$ EDTA, $1 \mathrm{mM}$ DTT ) containing varying concentrations of guanidinium chloride $(\mathrm{GdmCl})$ in the presence and absence of proteinase $\mathrm{K}\left(1 \mu \mathrm{g} \mathrm{ml}^{-1}\right)$. To determine luciferase activity, $2 \mu \mathrm{l}$ diluted RRL sample was withdrawn and mixed with $30 \mu 1$ Luciferase Assay System (Promega).

\section{IV.5.6. Cold Denaturation Method}

Fluc mutants were transcribed and translated in RRL at $25^{\circ} \mathrm{C}$ for 90 min followed by three-fold dilution in stop buffer containing $2 \mathrm{mM}$ cycloheximide. The reaction mixture in each 
case was distributed into 2 aliquots for determining luciferase activity at $20^{\circ} \mathrm{C}$ and $4{ }^{\circ} \mathrm{C}$ at different times. After $60 \mathrm{~min}$, the aliquots at $4{ }^{\circ} \mathrm{C}$ were shifted to $20^{\circ} \mathrm{C}$ to follow the change in luciferase activity.

\section{IV.6. In Vivo Characterization of Fluc Variants}

\section{IV.6.1. Cell Culture and Transfection}

HeLa (ATCC Number: CCL-2) and HEK 293T (ATCC Number: CCL-131) cells were maintained in Dulbecco's Modified Eagle's medium (DMEM; Biochrom) supplemented with $10 \%$ fetal bovine serum (FBS), 1\% penicillin-streptomycin (Gibco) and 1\% L-glutamine (Gibco) at $37^{\circ} \mathrm{C}$ in an atmosphere of $5 \% \mathrm{CO}_{2}$. To perform heat-stress experiments, cells were transiently transferred to an incubator maintained at $43^{\circ} \mathrm{C}$ for $2 \mathrm{~h}$. The stock solutions for small molecule compounds, 17-AAG, MG132 and PBT were prepared in DMSO. The compounds were diluted in DMEM medium and applied to cells for the indicated times.

Transient transfection of HeLa cells was done using Lipofectamine and PLUS reagent (Invitrogen) according to the manufacturer's protocol. We used $1 \mu \mathrm{g}$ DNA for transfection in 12well format. HEK 293T cells were transiently transfected using Lipofectamine 2000 reagent (Invitrogen). Here we used $800 \mathrm{ng}$ DNA for transfection in 12-well format. esiRNA reversetransfection experiments were performed with Lipofectamine RNAiMAX (Invitrogen). We used $300 \mathrm{ng}$ of esiRNA for transfection in 12-well format. Forty-eight hours after esiRNA transfection, cells were again transfected with Fluc constructs as described above. For DnaJB1 down-regulation experiments, HEK 293T cells stably expressing NES-FlucDM-EGFP were transfected with either DnaJB1 siRNA (Cat. No. L-012735-01-0005) or control siRNA \#3 (Cat. No. D-001210-03-05) using DharmaFECT transfection reagent as recommended by the manufacturer (Dharmacon). In each transfection protocol (plasmid, esiRNA or siRNA), the transfection reagent was diluted in Opti-MEM medium and was replaced with DMEM medium (without penicillin-streptomycin solution) after $3 \mathrm{~h}$ of application to cells. 


\section{IV.6.2. Protein Sample Preparation and Specific Activity}

\section{Measurements}

For specific activity measurements, cells were transiently transfected with Fluc variants as described above. Twenty four hours after transfection, cells were trypsinized and resuspended in DMEM medium. Cells were counted using Scepter ${ }^{\mathrm{TM}}$ Automated Cell Counter (Millipore) and 10,000 cells/well in $100 \mu 1$ DMEM medium were seeded in 96-well format. The remaining cells were centrifuged and washed twice with PBS to remove excess trypsin and DMEM. To prepare total cell protein for immunoblotting, equal number of cells were either boiled directly in SDS sample buffer or dissolved in SDT buffer (4\% (w/v) SDS, $100 \mathrm{mM}$ Tris-HCl (pH 7.6) and $0.1 \mathrm{M}$ DTT). To prepare soluble cell fractions, cells were resuspended in cell lysis buffer (50 mM Tris (pH 7.8), $150 \mathrm{mM} \mathrm{NaCl}, 1 \%$ (v/v) NP-40, $0.25 \%$ sodium deoxycholate, $1 \mathrm{mM}$ EDTA and 1 tablet protease inhibitor cocktail per $10 \mathrm{ml}$ ) and vortexed at regular intervals for $40 \mathrm{~min}$ on ice. The crude cell lysate was then centrifuged at $16,100 \mathrm{~g}$ for $30 \mathrm{~min}$ at $4^{\circ} \mathrm{C}$. The supernatant fraction was used for analysis of soluble luciferase protein amount. Pellets were dissolved in same volume of SDT buffer.

After $24 \mathrm{~h}$, the cells in 96-well plate were lysed by adding $50 \mu \mathrm{l}$ Steady-Glo Luciferase Assay System buffer (Promega) directly to the wells and incubated in dark at room temperature for 15 min. Luminescence was then recorded in a luminometer (Berthold Lumat LB9507) and measuring time was set to $2 \mathrm{sec}$. To determine specific activities, the luminescence values (Fluc activities) were divided by luciferase band intensity quantified from immunoblots by densitometry using the AIDA software.

\section{IV.6.3. Fluorescence Microscopy}

For preparing samples for fluorescence microscopy, cells were transiently transfected in 12-well format and $24 \mathrm{~h}$ later were seeded onto $12 \mathrm{~mm}$ round poly-L-lysine coverslips (BD Biocoat). Following day, the cells were given treatment (heat stress or addition of small molecules) and fixed with $4 \%$ paraformaldehyde. After fixation, cells were washed thrice with PBS and stained with DAPI (to stain DNA). DAPI staining was followed by three times washing with PBS and coverslips were then mounted on glass slides using Dako fluorescent mounting medium for fluorescence microscopy. 
Worms were fixed in ethanol and mounted on glass slides using Dako fluorescent mounting medium. Fluorescence imaging was performed on a Zeiss Axiovert 200M fluorescence microscope equipped with a Zeiss Axiocam HRM camera. Images were acquired using Axiovision Rel 4.7 software. Images were resized and brightness and contrast was adjusted in Adobe Photoshop CS5.1 software. The filter sets used for image acquisition were the following

\begin{tabular}{|c|c|c|c|c|}
\hline Channel & Filter Set No. & Beam Splitter & Excitation Filter & Emission Filter \\
\hline DAPI & 1 & FT395 & BP365/12 & LP397 \\
\hline EGFP & 38 & FT580 & BP470/40 & BP525/50 \\
\hline Cy3/mCherry & 15 & FT495 & BP546/12 & LP590 \\
\hline
\end{tabular}

For confocal micrographs (only of HEK 293T cells in this study), samples were prepared as described above. The confocal microscopy was performed in the MPI core facility using the Leica TCS SP2 confocal laser scanning microscope equipped with AOBS beam splitter. Images were acquired using the Leica HCX PL APO 63X oil immersion objective with numerical aperture of 1.4 and a resolution of 512 x 512. Virtual zoom for each confocal image was set to 4.00 and image acquisition and analysis was done using Leica TCS Analysis software (Leica).

For fluorescence immunocytochemistry, cells were fixed with $4 \%$ paraformaldehyde, followed by 3 times washing with PBS ( $\sim 5 \mathrm{~min}$ each). Cells were then treated with ice-cold methanol-acetone solution $(1: 1)$ and kept at $-20^{\circ} \mathrm{C}$ for $10 \mathrm{~min}$. After washing with PBS, cells were treated with 5\% skimmed milk solution containing $0.1 \%(\mathrm{v} / \mathrm{v})$ Triton-X-100 for $1 \mathrm{~h}$ at room temperature to block and permealize the cells. Primary antibody was diluted (1:500-1:1000) in blocking solution and cells were incubated with the antibody solution at $4{ }^{\circ} \mathrm{C}$ for overnight. Cells were extensively washed with PBS and incubated with fluorochrome-conjugated secondary antibody (diluted 1:200) in PBS/Triton for $2 \mathrm{~h}$ at room temperature in dark. For DAPI staining, cells were rinsed twice with PBS to remove excess secondary antibody and were stained with DAPI. DAPI staining was followed by three times washing with PBS and coverslips were then mounted on glass slides using Dako fluorescent mounting medium for fluorescence microscopy.

\section{IV.6.4. Live Cell Imaging}

For Live cell imaging, 7500 cells/well were grown in DMEM (without phenol red) in a $\mu$-Slide 8-well chambered dish coated with poly-L-lysine (IBIDI). Live cell imaging was 
performed using Perkin Elmer UltraVIEW Vox Spinning Disk system with a Leica DMI6000 B inverted microscope equipped with a Leica HCX PL APO 63X oil immersion objective with numerical aperture of 1.4. The system was also equipped with HAMAMATSU ImagEM camera with a resolution of 512 x 512 and with fully motorized XYZ stage for automated point visiting feature. During live cell imaging experiment, the cells were provided optimum growth conditions of $5 \%$ humidified $\mathrm{CO}_{2}$ and $37^{\circ} \mathrm{C}$ temperature. For live cell imaging of HEK 293T cells transiently co-expressing Fluc-EGFP variants and Htt-polyQ-mCherry constructs, image acquisition was started $24 \mathrm{~h}$ after transfection. To monitor the effect of proteasome inhibition on the stability of NES-FlucEGFP variants, live cell imaging of HEK 293T cells stably expressing NES-Fluc-GEFP or NES-FlucDM-EGFP was started soon after the addition of $5 \mu \mathrm{M}$ MG132 to the medium. Image acquisition and analysis was done using Volocity 6.0 (Perkin Elmer) software.

\section{IV.6.5. Generation of Stable Cell Lines}

In this study, stable cell lines of HEK 293T cells expressing NLS- and NES-Fluc-EGFP variants were generated. Before starting stable cell line generation, a kill curve of Neomycin (G418), used as a selection marker, to select transfected cells over untransfected cells was prepared. Untransfected HEK 293T cells were treated with increasing concentrations of G418 from $100-800 \mu \mathrm{g} / \mathrm{ml}$ for $\sim 10$ days till all cells were dead. The concentration of G418 used to select transfected cells was $400 \mu \mathrm{g} / \mathrm{ml}$.

Approximately $50 \times 10^{4}$ HEK 293 T cells were transfected with 1600 ng DNA of NLSand NES-Fluc-EGFP variants using Lipofectamine 2000 reagent (Invitrogen) as recommended by the manufacturer. Twenty four hours after transfection, cells were counted and $10^{4}$ cells were transferred to a new plate. A control plate containing $10^{4}$ untransfected cells was also prepared. Following day, the cells were treated with $400 \mu \mathrm{g} / \mathrm{ml} \mathrm{G418}$ and DMEM was replaced after every second day. The treatment of cells with G418 was continued till 95\% cells in control plate died and transfected cells started forming colonies. Individual colonies were then picked under sterile conditions, using pipette and microscope, and were transferred into 96 well plates. The cells were allowed to recover in DMEM without G418 and positive clones were selected by measuring luciferase activity and monitoring EGFP fluorescence in each case. Different clones of each Fluc construct were selected and stored until use. 


\section{IV.6.6. Cryo-Preservation of Mammalian Cell Lines}

To preserve mammalian cells, cells were trypsinized and resuspended in DMEM medium. The cell suspension was centrifuged at $1000 \mathrm{~g}$ for $5 \mathrm{~min}$ and supernatant was discarded to remove excess trypsin. The cell pellet was then resuspended in DMEM medium containing 10\% (v/v) DMSO, 20\% (v/v) FBS and $2 \mathrm{ml}$ aliquots were prepared in cryo-tubes. The cells were first transferred into an isopropanol filled cryo-preservation box at $-80^{\circ} \mathrm{C}$ for three days and subsequently transferred to liquid nitrogen tank.

For re-vitalizing frozen cells, an aliquot was withdrawn from liquid nitrogen tank and placed in a water bath at $37^{\circ} \mathrm{C}$. The cells were then seeded in a tissue culture plate in DMEM supplemented with $20 \%$ FBS. The cells were allowed to adhere to the plate and medium was changed with a fresh DMEM after they completely adhered to the plate bottom.

\section{IV.6.7. Generation of Transgenic C. elegans}

C. elegans were routinely maintained at $20^{\circ} \mathrm{C}$ on solid nematode growth medium (NGM) agar, seeded with E. coli strain OP50 as a food source. Transgenic C. elegans were generated by microinjection of worms (Bristol strain N2) with Fluc-EGFP expression vectors. Fluc-EGFP expression vectors $\left(25 \mu \mathrm{g} \mathrm{ml}^{-1}\right.$ ) together with the injection marker pRF4 (rol-6 (su1006), $125 \mu \mathrm{g}$ $\mathrm{ml}^{-1}$ ) were injected into the gonads of adult wild-type (N2) hermaphrodite worms. We selected transgenic F1 progeny on the basis of the roller phenotype (expressed from pRF4 marker). Individual transgenic F2 worms that were rollers with EGFP fluorescence were picked to establish independent lines. To achieve chromosomal integration, selected extrachromosomal lines were UV-light irradiated at energy of $300 \mathrm{~J}$ and integrated strains were backcrossed six times before using them in experiments. The following transgenic strains were generated and used in this study

FUH62 [marIs62 (P(unc-54)Fluc::EGFP)]

FUH134 [marls 134 (P(unc-54)FlucSM::EGFP)]

FUH135 [marls135 (P(unc-54)FlucDM::EGFP)]

FUH48 [marls48 (P(F25B3.3)Fluc::EGFP)]

FUH136 [marIs 136 (P(F25B3.3)FlucSM::EGFP)]

Neuron Specific Expression

FUH137 [marls 137 (P(F25B3.3)FlucDM::EGFP)] 


\section{IV.6.8. Reverse Transcriptase-PCR (RT-PCR) Experiments in C. elegans}

To perform RT-PCR experiments, we isolated total RNA from 600 FlucDM-EGFPexpressing worms by the trizol method. $1 \mu \mathrm{g}$ of total RNA was reverse transcribed to cDNA using the iScript Select cDNA Synthesis Kit (Bio-Rad) according to the guidelines of the manufacturer. The primers used in this experiment to amplify cDNA were the following.

\begin{tabular}{|l|l|}
\hline Primer & Sequence \\
\hline FlucDM-EGFP FOR & 5'-AGATGACGGGAACTACAAGACACG - 3' \\
\hline FlucDM-EGFP REV & 5'- GTGGTCTCTCTTTTCGTTGGGATC - 3' \\
\hline unc-54 FOR & 5'- ACGTGTTCGTGAGCTTCAATTCCAGG - 3' \\
\hline unc-54 REV & 5'-AGATGGCGATCTGATGACAGCGGC - 3' \\
\hline unc-119 FOR & 5'- AATGAGACGGAAGAGAATCTGC - 3' \\
\hline unc-119 REV & 5'- GATCATGTCGTCCATGAGTTGT - 3' \\
\hline
\end{tabular}

PCR amplification was carried out using three different dilutions of cDNA in $50 \mu$ l reaction volume and following PCR conditions were used.

\begin{tabular}{|l|c|c|}
\hline Reaction Step & Temp & Time \\
\hline Initial Denaturation & $95^{\circ} \mathrm{C}$ & $2 \mathrm{~min}$ \\
\hline Cycle Denaturation & $95^{\circ} \mathrm{C}$ & $30 \mathrm{sec}$ \\
\hline Annealing & $56^{\circ} \mathrm{C}$ & $30 \mathrm{sec}$ \\
\hline Extension & $72^{\circ} \mathrm{C}$ & $1 \mathrm{~min}$ \\
\hline Final Extension & $72^{\circ} \mathrm{C}$ & $5 \mathrm{~min}$ \\
\hline
\end{tabular}

$5 \mu \mathrm{l}$ of amplified PCR product was analyzed on $2 \%$ agarose gel at constant voltage of $100 \mathrm{~V}$. Experiments were repeated at least three times, and band intensities were quantified using AIDA image analyzer software. 


\section{Results}

\section{V.1. Generation of Conformationally Destabilized Firefly}

\section{Luciferase Mutants}

Luciferase from the American firefly Photinus pyralis (EC 1.13.12.7) (Fluc) catalyzes an oxidation reaction involving luciferin, ATP and molecular oxygen in the presence of a divalent magnesium ion to yield an electronically excited oxyluciferin species (Figure 13a). The decay of the excited-state oxyluciferin to the ground state emits a light in the yellow-green region of the visible spectrum with a quantum yield of nearly 100\% (Deluca, 1976; Seliger and Mc, 1960).

a

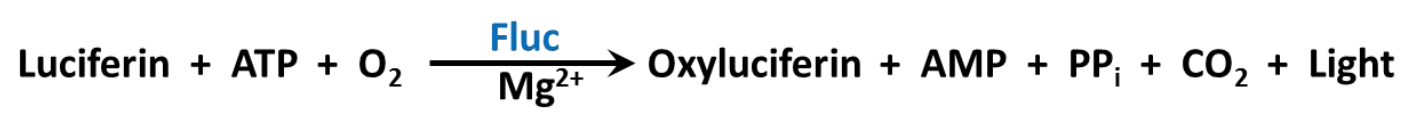

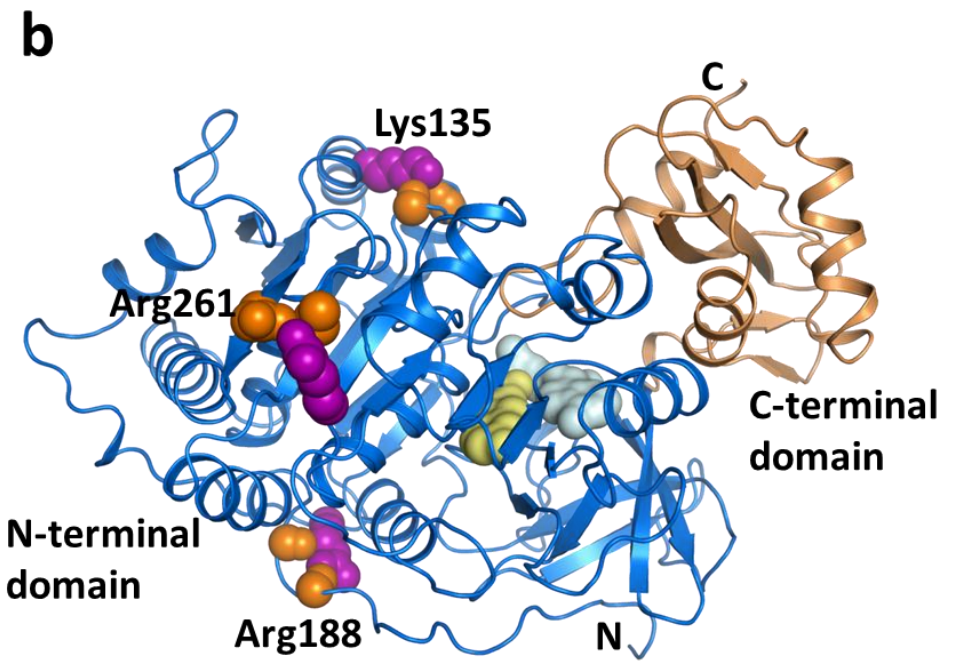

7. $K 135 Q, R 188 Q$

8. K135Q,R188K

9. $K 135 Q, R 261 Q$

1. $\mathrm{K} 135 \mathrm{Q}$

10. K135Q,R261K

2. $\mathrm{K} 135 \mathrm{M}$

11. $K 135 \mathrm{M}, \mathrm{R} 188 \mathrm{Q}$

3. $\mathrm{R} 188 \mathrm{Q}$

12. K135M, R188K

4. R188K

13. K135M,R261Q

5. R261Q

14. K135M,R261K

6. R261K

15. R188Q, R261Q

16. R188Q,R261K

17. R188K,R261Q

18. R188K,R261K

Figure 13: Design of conformationally destabilized Firefly luciferase (Fluc) variants.

(a) Fluc-mediated enzymatic reaction. (b) Crystal structure of Fluc (Protein Data Bank: 2d1r) (Nakatsu et al., 2006). Highlighted are N-terminal domain (blue) and C-terminal domain (gold). The residues chosen for mutations are highlighted in pink while their hydrogen bond interactors (Asp107 and Tyr109, Glu18 and Gly20, and Thr43 and Asn50) are shown in orange. Products of Fluc catalyzed reaction; AMP and oxyluciferin are shown in light blue and light green respectively, indicating the active site. Six single and twelve double mutants of Fluc are generated. The mutations R188Q and R188Q+R261Q in red are extensively characterized in this study. 
Fluc is a $\sim 60 \mathrm{kDa}$ protein and consists of two distinct domains, the large N-terminal domain (residues 1-421) and the small C-terminal domain (residues 422-544) (Conti et al., 1996) (Figure 13b). The N-terminal domain consists of a sub-domain (residues 1-190) of $22 \mathrm{kDa}$ that has been shown to already fold during translation (Frydman et al., 1999). This co-translational folding of the N-terminal sub-domain allows the rapid folding of the entire Fluc molecule upon release of the polypeptide chain from the ribosome. The $\mathrm{N}$-terminal domain is separated from the C-terminal domain by a wide cleft, and a flexible hinge connects these two domains. The activesite pocket for the substrates (ATP and luciferin) is constituted by highly conserved residues within this cleft. The C-terminal domain acts as a lid over the active site and during the course of the reaction it presumably seals the excited-state oxyluciferin from solvent-mediated quenching of luminescence (Conti et al., 1996) (Figure 13b).

To design increasingly destabilized mutants of Fluc, we mutated the large N-terminal domain by weakening the polar contacts between amino acids that are located distant in the Fluc sequence. The majority of the polar contacts are long-distance salt bridges and hydrogen bond interactions which often contribute substantially to the thermodynamic stability of the native fold in proteins (Matsui and Harata, 2007). To generate Fluc mutants with unchanged enzymatic activity in the folded state, we excluded the positions close to the substrate-binding pocket as well as positions at the domain-domain interface. We selected three positions (i) Lys135, (ii) Arg188 and (iii) Arg261 for mutagenesis, which are within hydrogen bond distance to Asp107 and Tyr109, Glu18 and Gly20, and Thr43 and Asn50, respectively. Using this strategy and a site directed mutagenesis approach, we generated six single mutants and twelve double mutants of Fluc as listed in Figure 13b. Fluc contains a C-terminal Ser-Lys-Leu (SKL) tripeptide that targets the protein to the peroxisomes (Gould et al., 1989). To retain Fluc in the cytosol, we replaced the SKL tripeptide by Ile-Ala-Val (IAV) which effectively blocks import of Fluc into peroxisomes (Sherf and Wood, 1994).

We first characterized the mutant proteins in vitro and then expressed them in cellular models and in $C$. elegans to validate their applicability as proteostasis sensors. Further, we tagged Fluc variants with appropriate signal sequences to study protein homeostasis in the cytosol and the nucleus of mammalian cells under different stress conditions. 


\section{V.2. In Vitro Characterization of Fluc Mutants}

\section{V.2.1. Functional Characterization of Fluc Mutants in RRL}

\section{V.2.1.1. Thermal Instability of Fluc Mutants}

In order to test the conformational and functional stability of the Fluc mutants in vitro, we used rabbit reticulocyte lysate (RRL). The RRL system contains all essential cellular components necessary for transcription, translation and protein folding and therefore allows the rapid production of proteins in vitro. We translated Fluc variants in RRL at $30^{\circ} \mathrm{C}$ for $90 \mathrm{~min}$. Following synthesis, we terminated the translation reaction by addition of buffer containing 2 $\mathrm{mM}$ cycloheximide $(\mathrm{CHX})$. This step was followed by incubation of the RRL reaction mixtures at different temperatures and measurement of Fluc activity as a function of time (Figure 14).

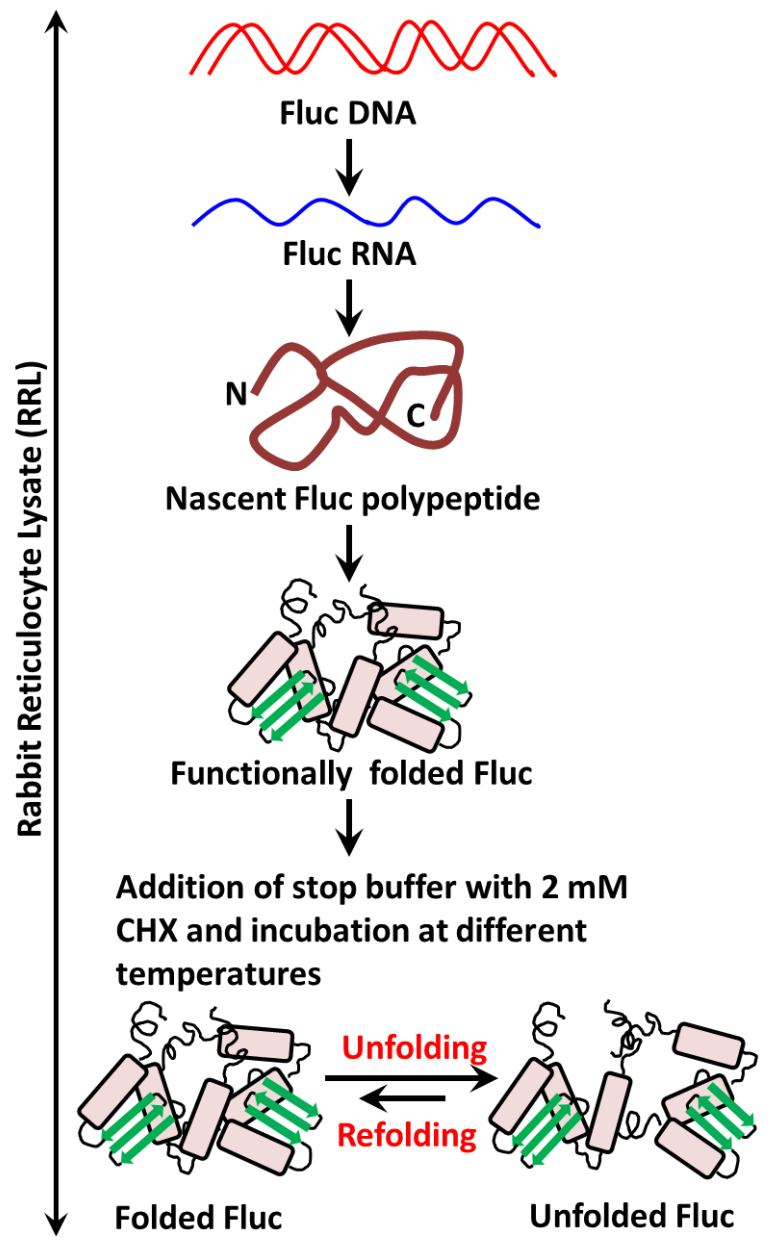

Figure 14: Schematic diagram illustrating the methodology used to characterize Fluc mutant proteins in RRL.

Fluc DNA was transcribed and translated in RRL at $30^{\circ} \mathrm{C}$ for $90 \mathrm{~min}$ to obtain Fluc protein. The translation reaction was stopped by addition of stop buffer containing $2 \mathrm{mM}$ cycloheximide (CHX) and tubes were incubated at different temperatures. At regular time intervals, RRL samples were withdrawn and Fluc activity was measured for each Fluc variant. 


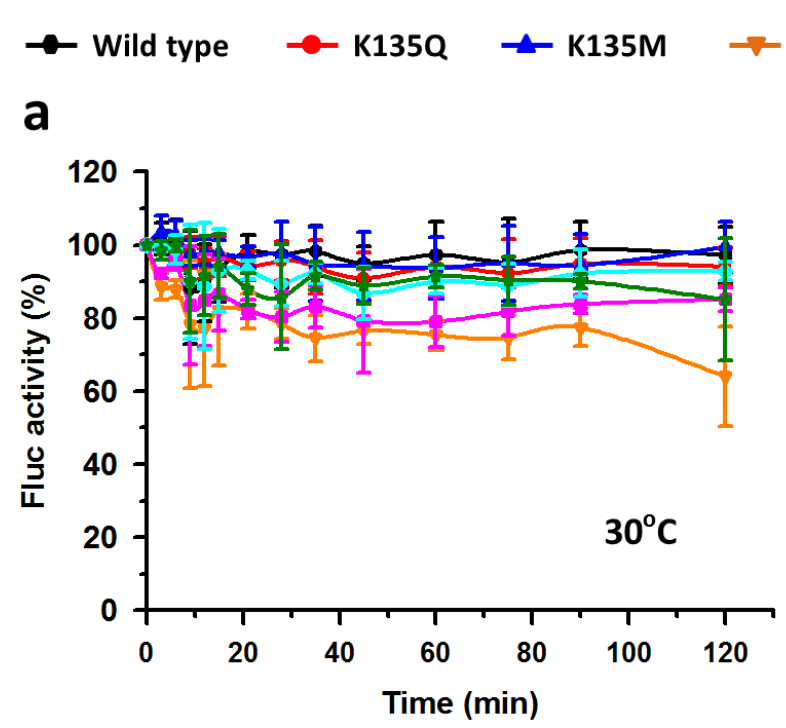

b

R261K

C
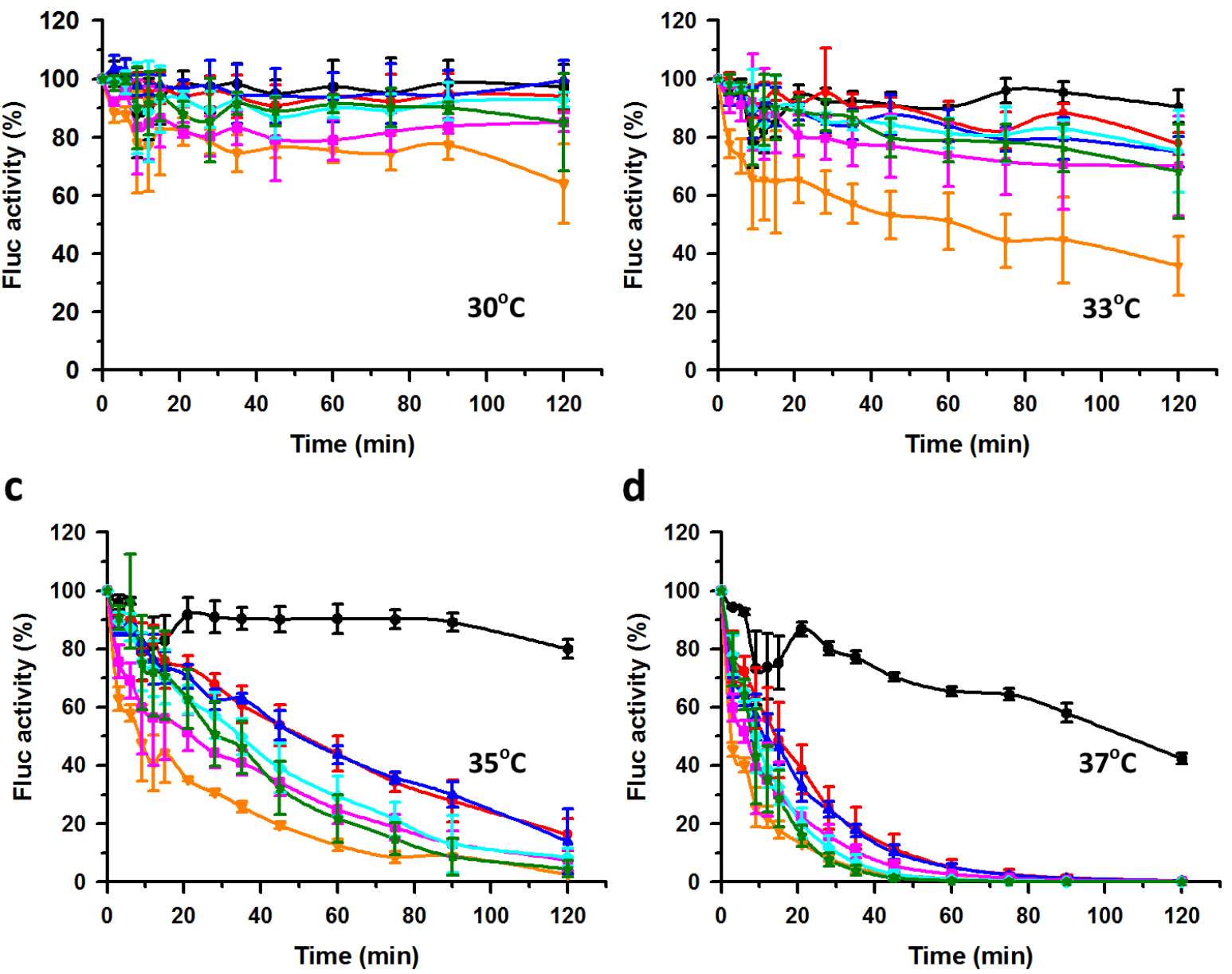

d



Figure 15: Temperature dependent loss of functionality of Fluc single mutants.

Fluc single mutants were translated in RRL at $30^{\circ} \mathrm{C}$ for $90 \mathrm{~min}$ as shown in scheme (Figure 14). The translation was inhibited and enzymatic activity of Fluc variants was followed from $30^{\circ} \mathrm{C}$ to $37^{\circ} \mathrm{C}$ (a-d) by measuring their activity at the times indicated. Fluc activity is expressed in percentage of the activity measured immediately after translation at $30^{\circ} \mathrm{C}$ (set to $100 \%$ ). Error bars indicate s.d., $n=3$.

At lower temperatures, $30^{\circ} \mathrm{C}$ and $33^{\circ} \mathrm{C}$, all Fluc single mutants except mutant $\mathrm{R} 188 \mathrm{Q}$ were enzymatically active (Figure 15a, b). The single mutant R188Q had lost 30\% activity at $30^{\circ} \mathrm{C}$ and $\sim 65 \%$ activity at $33^{\circ} \mathrm{C}$ in $120 \mathrm{~min}$. At $35^{\circ} \mathrm{C}$, wild-type Fluc was still active while Fluc single mutants lost 50-80\% of their enzymatic activities within 60 min (Figure 15c). Incubation of Fluc variants at $37^{\circ} \mathrm{C}$ had a drastic effect on the activities of the mutants which lost more than $90 \%$ of activity in 60 min, compared to wild-type Fluc that lost 35\% of the original activity in 
60 min (Figure 15d). Therefore, among all the single mutations, the mutation R188Q had the most pronounced effect on the thermodynamic stability of luciferase enzyme.


Figure 16: Temperature dependent loss of functionality of Fluc double mutants.

Fluc double mutants were translated in RRL at $30^{\circ} \mathrm{C}$ for $90 \mathrm{~min}$, followed by inhibition of translation and incubation at $30^{\circ} \mathrm{C}$ to $37^{\circ} \mathrm{C}$ (a-d) as in Figure 14. Fluc activity was measured at the times indicated and expressed in percentage of the activity measured immediately after translation at $30^{\circ} \mathrm{C}$ (set to $100 \%$ ). Error bars indicate s.d., $\mathrm{n}=3$.

Similar to Fluc single mutants, we also characterized double mutants of Fluc in RRL at different temperatures. At $30^{\circ} \mathrm{C}$, all double mutants showed gradual decline in their enzymatic activities with time. The double mutants containing R188Q lost $~ 50 \%$ of their original activities after incubation for 120 min whereas those that contain R188K lost 30-40\% activity (Figure 16a). At $33^{\circ} \mathrm{C}$, the double mutants containing R188Q lost $80 \%$ of the original activity within 30 $40 \mathrm{~min}$ in comparison to other mutants that lost same amount of activity in more than $60 \mathrm{~min}$ 
(Figure 16b). All Fluc double mutants lost 80\% of original enzymatic activity within 20 min of incubation at $35^{\circ} \mathrm{C}$ and within $5 \mathrm{~min}$ at $37^{\circ} \mathrm{C}$ (Figure 16c, d). Among all the double mutants that were functionally characterized in RRL, the mutant K135M+R188K was most stable whereas the mutants containing R188Q were the least stable. As expected, the Fluc double mutants were more destabilized relative to the single mutants from which they were derived. The loss of enzymatic activity of Fluc single and double mutants at increasing temperatures indicates that they have distinct thermodynamic stabilities at different temperatures.

\section{V.2.1.2. Specific Activities of Fluc Mutants in RRL}

To investigate whether the loss of activities of Fluc mutant proteins at $30^{\circ} \mathrm{C}$ is due to their functional inactivation or due to the reduction in protein amount, we determined the specific activities (activities relative to the protein amount) of all the Fluc variants (Figure 17).

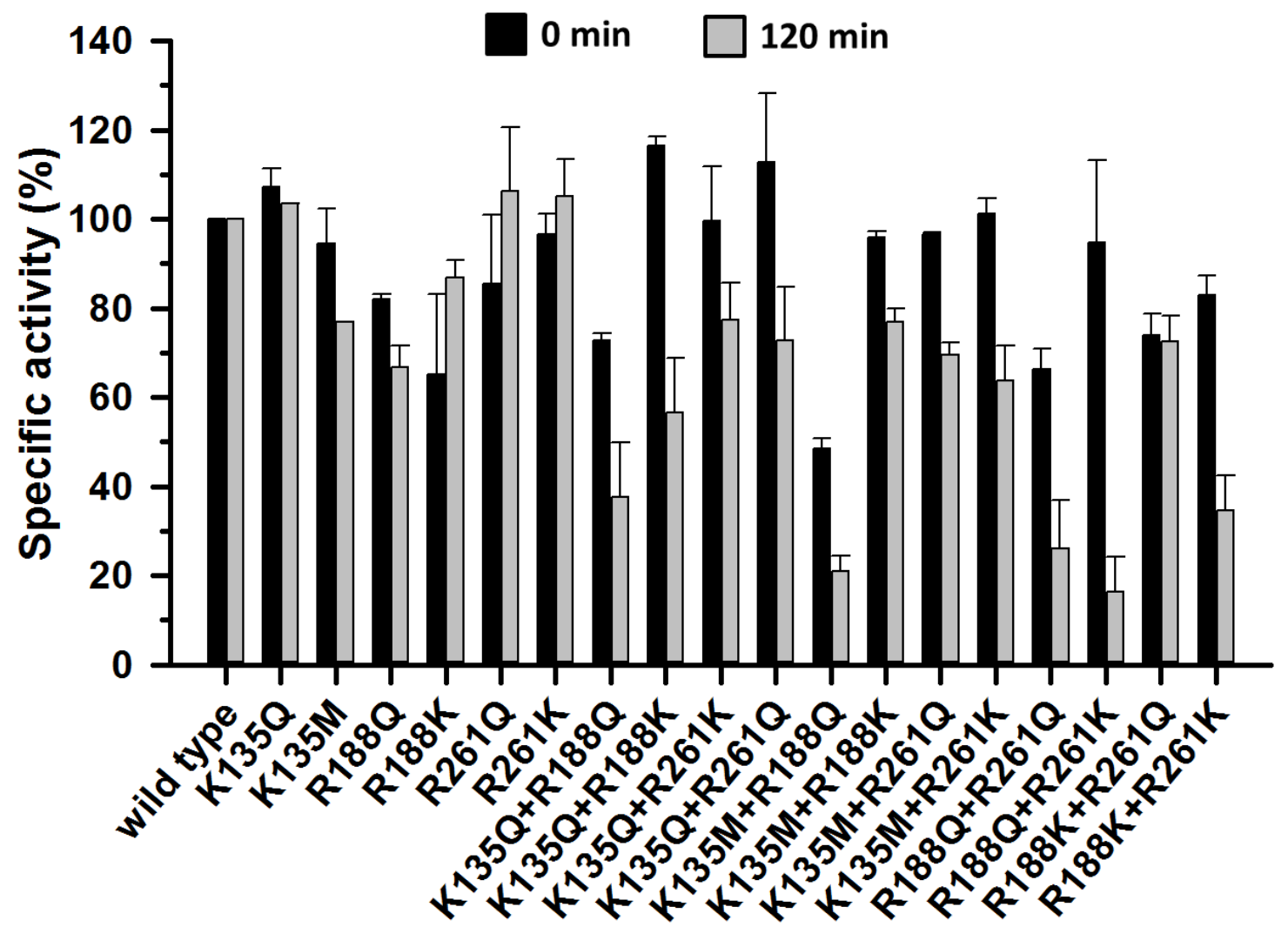

Figure 17: Specific luminescence activity of the Fluc variants in RRL at $30^{\circ} \mathrm{C}$.

Wild type Fluc and its mutant counterparts were translated in RRL at $30^{\circ} \mathrm{C}$ for $90 \mathrm{~min}$. Immediately after translation $\left(0 \mathrm{~min}\right.$, black bars) and after $2 \mathrm{~h}$ of incubation at $30^{\circ} \mathrm{C}(120 \mathrm{~min}$, grey bars $)$, the enzyme activities were measured and protein amounts were determined by immunoblotting of total RRL fractions. Specific activity was calculated by dividing the enzyme activity with the protein amount. Specific activity of wild-type Fluc was set to $100 \%$ and used to normalize for the mutants. Error bars indicate s.d., n=3. 
At the 0 min time point, just after translation, the relative specific activities of all the Fluc mutants, except K135M+R188Q, were $70-80 \%$ of the specific activity of the wild-type protein, indicating that the initial folding of the mutant proteins was highly efficient at $30^{\circ} \mathrm{C}$. However, upon incubation for $2 \mathrm{~h}$ at $30^{\circ} \mathrm{C}$, the Fluc single mutants except R188Q, retained $\sim 80 \%$ of specific activity of wild-type Fluc. The specific activities of Fluc double mutants showed variation, with mutants carrying R188Q mutation losing 70-80\% of their specific activity. Thus, among the single mutants, the mutant R188Q and among the double mutants, the mutants containing R188Q were the least stable. This observation was in agreement with the denaturation profile of Fluc single and double mutants at $30^{\circ} \mathrm{C}$ for $2 \mathrm{~h}$ (Figure 15a, 16a) and suggests that the mutant proteins were able to fold properly into their functional active state but weren't able to maintain this state and were denatured.


Figure 18: Thermal stability of wild-type (Fluc), single mutant R188Q (FlucSM) and double mutant R188Q+R261Q (FlucDM) selected for further characterization.

(a-c) Temperature-dependent loss of activity of Fluc (a), FlucSM (b) and FlucDM (c) measured at the indicated times and expressed as a percentage of the activity measured immediately after translation (set to $100 \%$ ). Proteins were translated in RRL (90 min at $30^{\circ} \mathrm{C}$ ), followed by inhibition of translation and incubation at $20-37^{\circ}$ C. (d) Specific activity of Fluc, FlucSM and FlucDM. The data points were taken from Figure 17. Error bars indicate s.d., $n=3$. 
For further in vitro and in vivo studies, we selected the single mutant R188Q and the double mutant R188Q+R261Q on the basis of their stability and examined them in detail. For future reference, the wild-type luciferase is referred as Fluc, the single mutant R188Q as FlucSM and the double mutant R188Q+R261Q as FlucDM. We additionally determined the enzymatic activities of these three Fluc variants upon incubation for $2 \mathrm{~h}$ at $20^{\circ} \mathrm{C}$ and $25^{\circ} \mathrm{C}$ in RRL (Figure 18a-c). While both Fluc and FlucSM were stable at these temperatures, FlucDM was unstable at $25^{\circ} \mathrm{C}$ relative to $20^{\circ} \mathrm{C}$. Thus, as summarized in Figure 18 , among these three Fluc variants wildtype Fluc was the most stable, FlucSM was less stable and FlucDM was the least stable. The results also suggest that polar contacts mediated by Arg188 with Glu18 and Gly20 and by Arg261 with Thr43 and Asn50 in Fluc are essential for maintaining its thermodynamic stability.

\section{V.2.1.3. Co-translational Folding Efficiency of Fluc Variants}

Using a RRL system, it has been shown before that the N-terminal sub-domain of Fluc undergoes co-translational folding which facilitate the rapid folding of the entire Fluc polypeptide upon its release from the ribosome (Frydman et al., 1999). Therefore we checked the effect of the mutations on co-translational folding. We monitored the luciferase activity at regular time intervals, from start of the reaction in RRL at $30^{\circ} \mathrm{C}$ to its completion after $90 \mathrm{~min}$ (Figure 19).



We observed a delay of $\sim 15 \mathrm{~min}$, corresponding to the time required for transcription and translation, in the read out of luciferase activity of the three Fluc variants. All Fluc variants 
reached saturation in activity after $45 \mathrm{~min}$, presumably due to the depletion of components involved in transcription and translation. Wild-type Fluc took $\sim 22$ min to reach 50\% of its maximum enzymatic activity compared to FlucSM and FlucDM which took 30 min, suggesting that the mutations have affected the co-translational folding efficiency of the Fluc variants.

\section{V.2.2. Structural Characterization of Fluc Variants in RRL V.2.2.1. Sensitivity of Fluc Variants to Proteinase K}

The results obtained from the previous experiments showed that the mutations affected the functional activity of the Fluc mutants. This could be due to their inability to reach native state or due to structural stability. Therefore, in order to obtain information about the effect of mutations on the structural flexibility of the Fluc variants, we performed proteinase $\mathrm{K}$ assays. Proteinase $\mathrm{K}$ is a serine protease that exhibits very broad cleavage specificity, cleaving peptide bonds adjacent to the carboxylic group of aliphatic and aromatic amino acids (Ebeling et al., 1974). Since completely folded proteins are less accessible to cleavage by proteinase K compared to unfolded and/or denatured proteins, the proteinase $\mathrm{K}$ assay can be used to obtain qualitative information about the structural integrity of proteins.

We compared the sensitivity of the newly translated Fluc mutant proteins to proteinase K by measuring the enzymatic activities of samples treated with the protease at $20^{\circ} \mathrm{C}$ (Figure 20a). In control reactions, without proteinase $\mathrm{K}$, there was no change in the enzymatic activities of the Fluc variants. In samples treated with proteinase K, luciferase activity decreased with a half-time $\left(t_{1 / 2}\right)$ of $\sim 20$ min for FlucSM and $\sim 7$ min for FlucDM. Proteinase $K$ treatment had no effect on the activity of Fluc. Moreover, digestion profiles of the Fluc mutant proteins as detected by immunoblotting showed a gradual decrease in their band intensities with time upon proteinase $\mathrm{K}$ addition (Figure 20b). This observation suggests that proteinase $\mathrm{K}$ has degraded the Fluc mutant proteins which corresponds to a loss of their enzymatic activities with time. Hence, these results strongly indicate that Fluc mutants are conformationally flexible and structurally destabilized relative to wild-type Fluc. 




\section{Dashed lines: +Proteinase $\mathrm{K}$}

$\triangle$ FlucDM

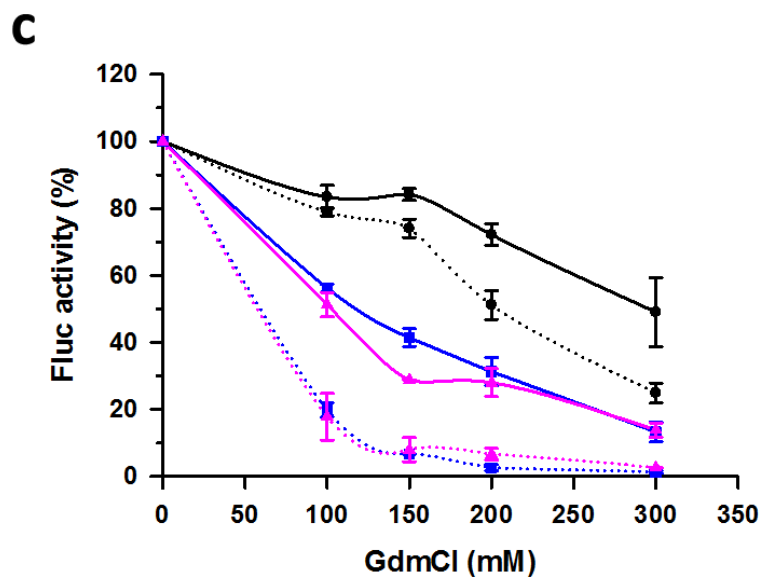

b

Fluc

FlucSM

FlucDM
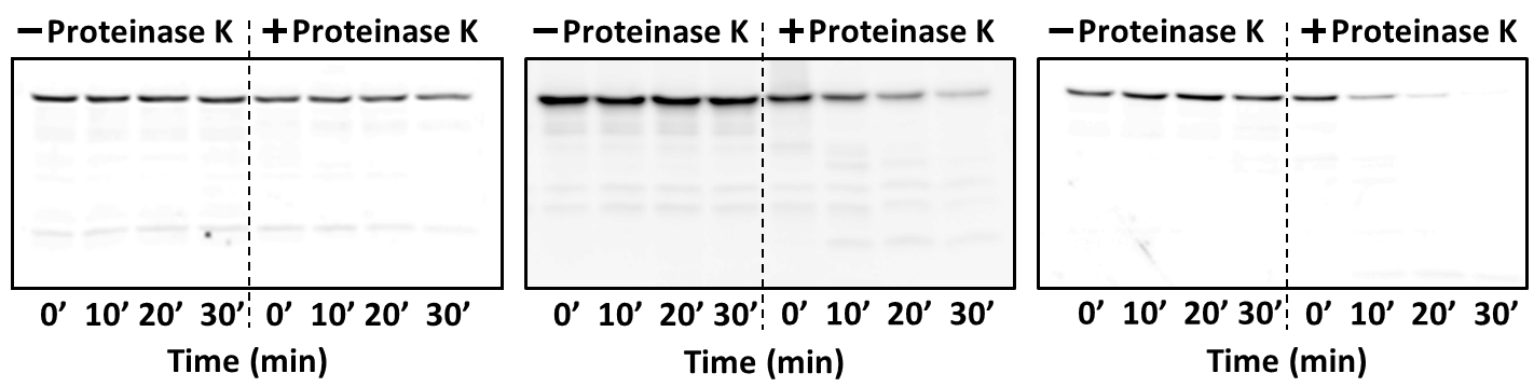

Figure 20: Assessment of conformational flexibility of Fluc variants.

Fluc mutant proteins are structurally destabilized. (a) Proteinase K sensitivity of Fluc variants in RRL at $20^{\circ} \mathrm{C}$. Proteins were translated in RRL $\left(90 \mathrm{~min}\right.$ at $30^{\circ} \mathrm{C}$ ), followed by inhibition of translation. The newlytranslated proteins were subjected to limited proteolysis by proteinase $\mathrm{K}$ at $20^{\circ} \mathrm{C}$. Enzymatic activity was measured at the times indicated and expressed in percentage of the activity measured immediately after translation (set to 100\%). (b) Proteinase $\mathrm{K}$ digestion profiles of the Fluc variants detected by immunoblotting with anti-Fluc antibody. (c) Proteinase K sensitivity of Fluc variants in presence of increasing concentration of guanidinium chloride. Protein samples were treated in the same manner as in (a). Error bars indicate s.d., $n=3$.

\section{V.2.2.2. Sensitivity of Fluc Variants to Guanidinium Chloride Mediated}

\section{Denaturation}

To obtain more insight into the structural instability of the Fluc mutant proteins, we studied their unfolding in vitro in the presence of the chaotropic denaturant guanidinium chloride $(\mathrm{GdmCl})$. $\mathrm{GdmCl}$ causes denaturation of proteins by disrupting non-covalent interactions (such as ionic interactions, hydrogen bonds etc.) and at high concentration (6 M), it leads to complete 
unfolding of proteins to random coil structures (O'Brien et al., 2007; Tanford, 1970). Therefore, $\mathrm{GdmCl}$ mediated denaturation can be used to study protein unfolding and folding intermediates. Thus, we incubated newly synthesized Fluc mutant proteins in RRL in increasing concentrations of $\mathrm{GdmCl}$ and measured their activities at $20^{\circ} \mathrm{C}$ (Figure 20c).

Compared to Fluc, which lost $\sim 50 \%$ of its original activity in $300 \mathrm{mM} \mathrm{GdmCl}$, we observed a rapid decrease in the activities of FlucSM and FlucDM at GdmCl concentration less than $150 \mathrm{mM}$, suggesting rapid unfolding of the proteins. The addition of proteinase $\mathrm{K}$ in the reaction mixture containing $\mathrm{GdmCl}$ further resulted in complete loss of enzymatic activities of Fluc mutants at $150 \mathrm{mM} \mathrm{GdmCl}$ while the Fluc remained stable under these conditions. Taken together, the sensitivity of Fluc mutants towards proteinase $\mathrm{K}$ and $\mathrm{GdmCl}$ suggest that they are structurally more destabilized than wild-type Fluc.

\section{V.2.2.3. Sensitivity of Fluc Variants to Cold Denaturation}

Following the proteinase $\mathrm{K}$ assay and the $\mathrm{GdmCl}$ mediated denaturation; we investigated the effect of low temperature on the structural and functional properties of Fluc and its variants. The rationale behind this experiment was that some proteins are sensitive to denaturation at low temperature, a phenomenon called "cold denaturation of proteins". Although the exact underlying mechanism of this phenomenon is still unclear, some studies have proposed a role of non-polar groups in unfolding of proteins due to cold denaturation (Dias et al., 2010; Privalov, 1990). From a thermodynamic perspective, the hydration of non-polar groups in a protein is favorable as this releases Gibb's energy of hydration, which is negative, and increases in magnitude at lower temperatures. Consequently, a tightly folded protein with buried hydrophobic residues unfolds at low temperature to expose these groups to water.

To investigate whether structurally destabilized Fluc variants are also prone to cold denaturation, we incubated the RRL reaction mixtures with newly translated Fluc mutant proteins at $4^{\circ} \mathrm{C}$ and measured luciferase activities at different times (Figure 21a). While wildtype Fluc was stable, FlucSM and FlucDM gradually lost 20\% and 60\% of their respective activities upon incubation at $4^{\circ} \mathrm{C}$ for $1 \mathrm{~h}$. This suggests that the mutant proteins due to their structural instability might have undergone rapid unfolding and presumably exposed their hydrophobic groups to the solvent. The subsequent incubation of the reaction mixtures from $4^{\circ} \mathrm{C}$ to $20^{\circ} \mathrm{C}$ after 60 min rapidly increased the activities of both FlucSM and FlucDM, indicating that 
the proteins immediately regained their conformations. No change in enzymatic activities of Fluc variants was observed at $20^{\circ} \mathrm{C}$. We obtained similar results in specific activity measurements of Fluc variants at $4^{\circ} \mathrm{C}$ and $20^{\circ} \mathrm{C}$ (Figure 21b). The sensitivity of FlucSM and FlucDM to low temperature supports the previous results that they are conformationally flexible relative to Fluc.



b

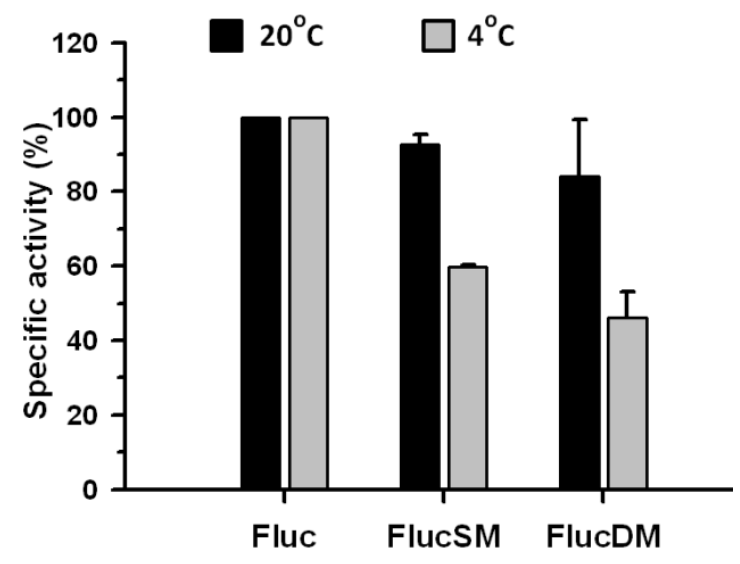

Figure 21: Fluc mutant proteins are sensitive to cold denaturation.

(a) Proteins were translated in RRL $\left(90 \mathrm{~min}\right.$ at $\left.30^{\circ} \mathrm{C}\right)$, followed by inhibition of translation. The newlytranslated proteins were incubated at either $20^{\circ} \mathrm{C}$ (solid lines) or at $4^{\circ} \mathrm{C}$ (dashed lines). Enzymatic activity was measured at the times indicated and is plotted as percentage of the activity measured immediately after translation (set to $100 \%$ ). The shift of samples from $4^{\circ} \mathrm{C}$ to $20^{\circ} \mathrm{C}$ after $60 \mathrm{~min}$ is indicated by a dashed line. (b) Specific activity of Fluc variants at $20^{\circ} \mathrm{C}$ and $4^{\circ} \mathrm{C}$ after 60 min incubation in RRL. Specific activity of wild-type Fluc was set to $100 \%$ and used to normalize for the mutants. Error bars indicate s.d., $n=3$.

\section{V.3. In Vivo Characterization of Fluc Variants}

\section{V.3.1. Chaperone Dependence of Fluc Variants in vivo}

Many studies have elucidated the role of chaperones, both in vivo and in vitro, in folding and refolding of Fluc (Frydman et al., 1994; Nimmesgern and Hartl, 1993; Schroder et al., 1993; Sharma et al., 2010; Thulasiraman and Matts, 1996). Therefore, in order to assess the stability of Fluc variants in vivo, we first determined their specific activities in HeLa cells (transformed epithelial human cell line derived from cervix). We transiently expressed Fluc, FlucSM and FlucDM at a concentration of $\sim 2-3 \mu \mathrm{g}$ luciferase protein per milligram total cell lysate. Compared to Fluc, the specific activities of FlucSM and FlucDM were 60\% and 40\% 
respectively (Figure 22a), suggesting that in HeLa cells, a substantial fraction of the mutant protein was functionally less active and misfolded. To assess the role of chaperones, we downregulated Hsc70 (HSPA8), a major constitutive chaperone required for folding of Fluc (Frydman et al., 1994), by RNAi. Down-regulation of HSPA8 by 50-70\% (Figure 22b) further decreased the specific activity of the Fluc variants (Figure 22a), presumably due to compromised folding of the mutant proteins in the absence of Hsc70 in cells. This result supports the previous findings that Fluc and its variants are dependent on Hsc70 (HSPA8) for their folding.

a

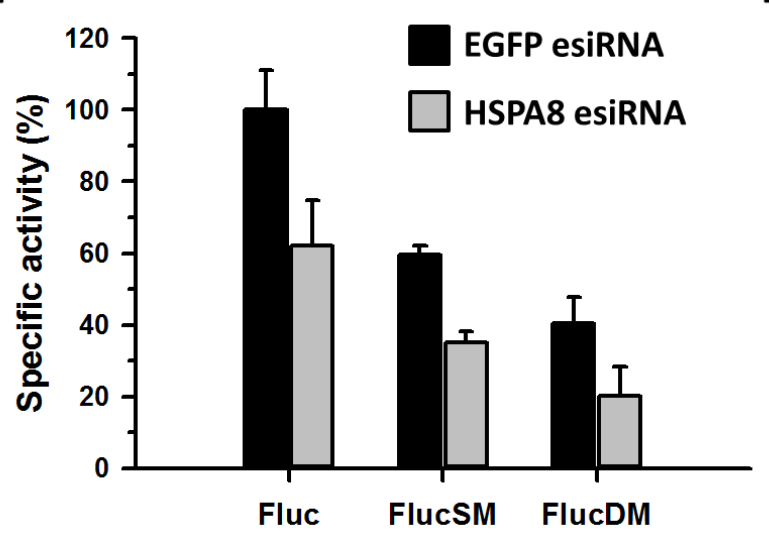

b

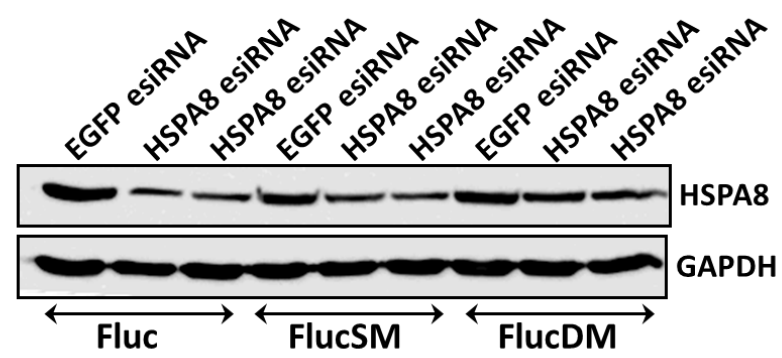

Figure 22: Chaperone dependence of Fluc variants in HeLa cells.

(a) Fluc, FlucSM and FlucDM were expressed for $48 \mathrm{~h}$ in HeLa cells treated with control esiRNA against EGFP (black bars) or esiRNA against HSPA8 (which encodes Hsc70) (grey bars). Specific activities of Fluc variants in soluble extracts were normalized to wild-type Fluc in control cells (set to 100\%). Error bars indicate s.d., $\mathrm{n}=3$. (b) A representative immunoblot showing the levels of HSPA8 (Hsc70) protein in samples treated with EGFP esiRNA or HSPA8 esiRNA. HSPA8 was probed with anti-Hsc70 antibody and GAPDH was used as a loading control.

\section{V.3.2. Generation and Characterization of Fluc-EGFP Variants in RRL}

In order to study different aspects of proteostasis in a cell culture model system, we tagged the Fluc variants with EGFP at the C-terminus. An EGFP tag was added to Fluc variants in order to monitor their cellular distribution under different stress conditions. To check the effect of the EGFP tag $(\sim 30 \mathrm{kDa})$ on the intrinsic stability of Fluc, FlucSM and FlucDM, we performed a functional and structural characterization of the respective fusion proteins in RRL as done for the proteins without the EGFP tag. Fluc-EGFP variants followed the same denaturation kinetics (Figure 23) and showed a similar digestion profile to proteinase K treatment (Figure 24) 
as their counterparts without the EGFP tag. Thus, Fluc-EGFP variants have similar functional and structural properties as variants without the tag and the EGFP tag has no effect on the stability of these fusion proteins. We therefore performed all the following experiments in vivo with the Fluc-EGFP variants.



Figure 23: Temperature dependent loss of enzymatic activity of Fluc-EGFP, FlucSMEGFP and FlucDM-EGFP.

Proteins were translated in rabbit reticulocyte lysate at $30^{\circ} \mathrm{C}$ for $90 \mathrm{~min}$, followed by inhibition of translation and incubation at $30^{\circ} \mathrm{C}$ to $37^{\circ} \mathrm{C}$. Fluc activity was measured at the times indicated and expressed as percentage of the activity measured immediately after translation at $30^{\circ} \mathrm{C}$ (set to $100 \%$ ). Error bars indicate s.d., $\mathrm{n}=3$. 


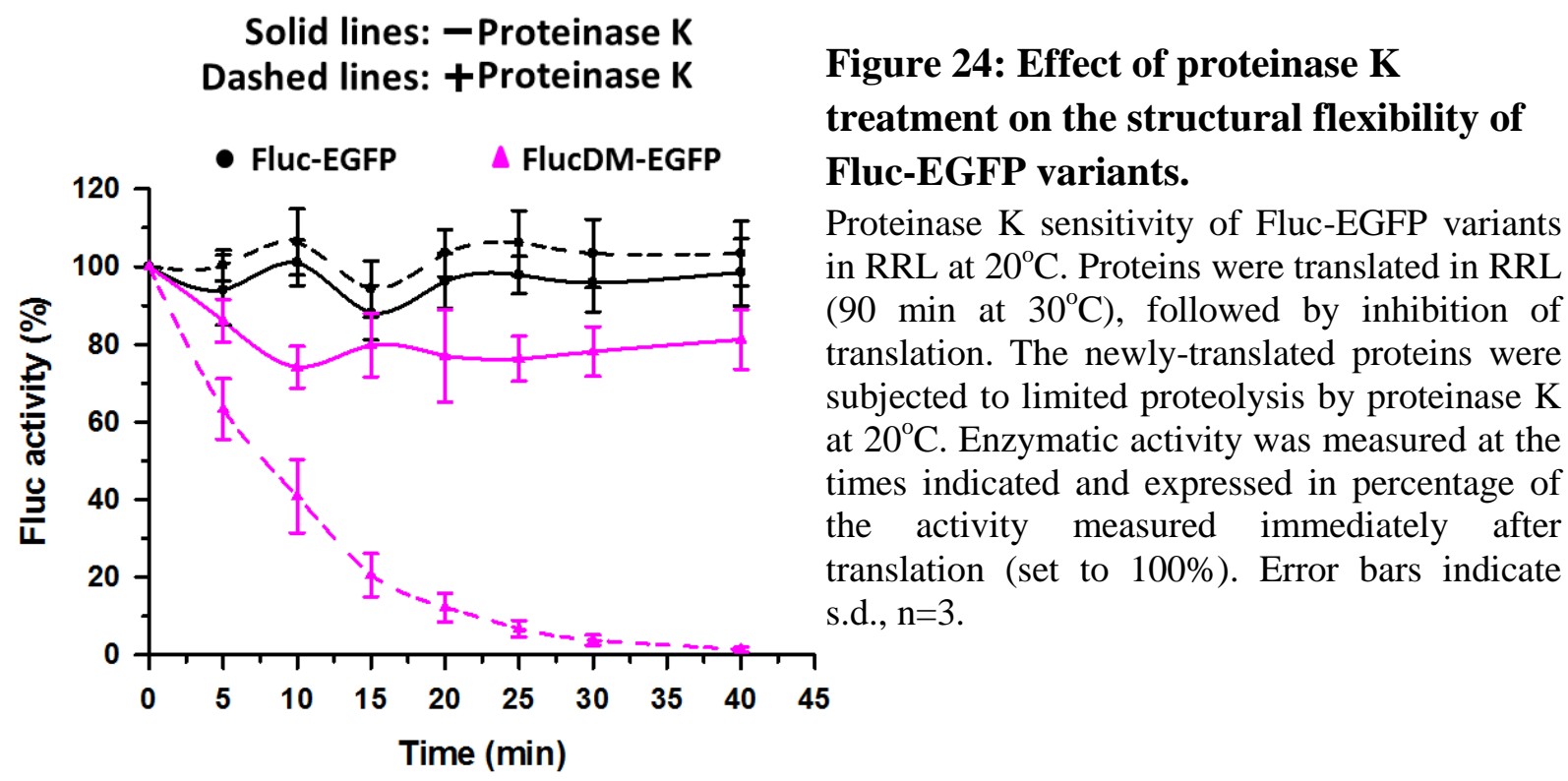

\section{V.3.3. Thermal Stability of Fluc-EGFP Variants in vivo}

To assess the sensitivity of Fluc-EGFP variants to high temperature in vivo, we subjected HeLa cells transiently expressing the Fluc-EGFP sensors to heat stress. At physiological temperature of $37^{\circ} \mathrm{C}$, all three Fluc-EGFP variants displayed a diffuse cellular distribution (Figure 25). The proteins were mainly cytosolic but they also showed some nuclear staining. However, in $~ 5 \%$ of cells expressing FlucDM-EGFP, a small number of green fluorescent foci were observed in the cytosol. This observation augmented the result from the specific activity measurements in vivo, supporting the conformational instability of FlucDM. When HeLa cells were subjected to heat stress at $43^{\circ} \mathrm{C}$ for $2 \mathrm{~h}$, Fluc-EGFP maintained its diffuse distribution in all cells while $\sim 45 \%$ of the FlucSM-EGFP-expressing cells and $\sim 75 \%$ of the FlucDM-EGFPexpressing cells showed inclusions in the cytosol. Upon recovery from heat stress $\left(2 \mathrm{~h}\right.$ at $\left.37^{\circ} \mathrm{C}\right)$, only $15-20 \%$ of FlucSM-EGFP-expressing cells and about 30\% of FlucDM-EGFP-expressing cells retained visible aggregates. This observation suggests that during recovery of cells, dissociation or degradation of aggregates had occurred. To exclude the contribution of newly synthesized protein to this process, we inhibited translation by addition of cycloheximide (CHX) to the cells immediately after the heat stress. Upon recovery of cells from heat stress, increased diffuse staining of the mutant proteins was observed (Figure 25). 


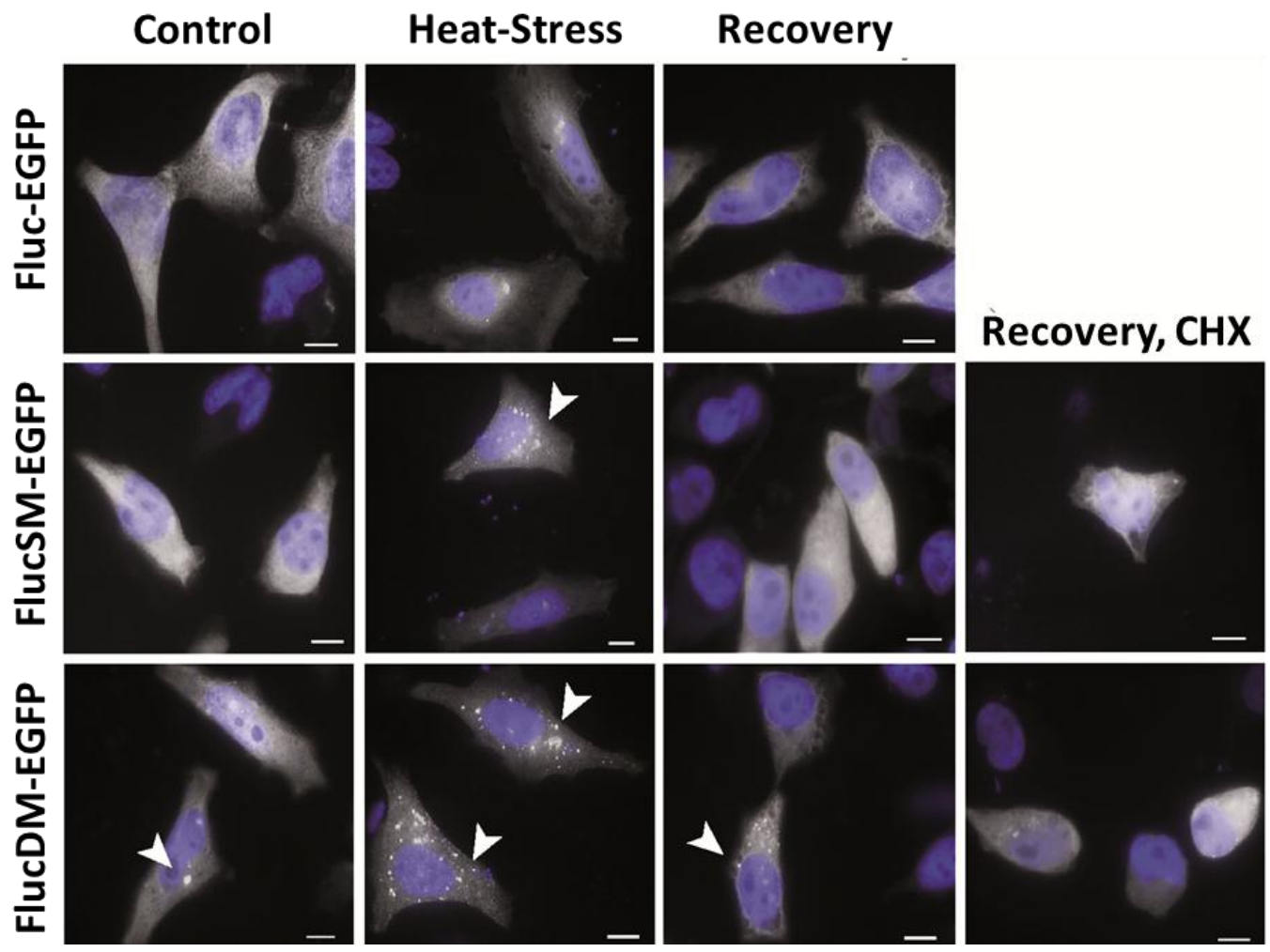

Figure 25: Thermal stability of Fluc variants in HeLa cells.

Representative fluorescence micrographs of HeLa cells expressing Fluc-EGFP, FlucSM-EGFP or FlucDM-EGFP under normal conditions at $37^{\circ} \mathrm{C}$ (control), after heat stress for $2 \mathrm{~h}$ at $43^{\circ} \mathrm{C}$ and after recovery (from heat stress) for $2 \mathrm{~h}$ at $37^{\circ} \mathrm{C}$ with or without cycloheximide (CHX). EGFP fluorescence is shown in white. Arrowheads indicate aggregates. Nuclei were stained with DAPI (blue). Scale bars correspond to $10 \mu \mathrm{m}$.

The observations were further supported by measurement of the specific activities of the Fluc-EGFP variants either immediately after heat stress $\left(43^{\circ} \mathrm{C}, 2 \mathrm{~h}\right)$ or upon subsequent recovery of the cells $\left(37^{\circ} \mathrm{C}, 2 \mathrm{~h}\right)$ in presence or absence of cycloheximide (Figure 26a). Immediately after heat stress, we could not detect any luciferase activity in HeLa cell lysates (in all three Fluc variants) owing to denaturation of the proteins. During recovery in the presence of CHX, FlucEGFP variants were able to regain $50-70 \%$ of their original specific activity, suggesting that Fluc-EGFP variants were able to refold. The result is in agreement with the microscopy experiment where dissociation of aggregates had occurred and diffuse staining of EGFP fluorescence was observed (Figure 25). During recovery in the absence of CHX, the specific activities of all Fluc variants exceeded those in control cells (without heat stress). This effect may be attributed to HSF1 mediated induction of HSPs during heat stress which may have 
resulted in more chaperone capacity and consequently increased folding efficiency of newly synthesized Fluc-EGFP proteins.

a

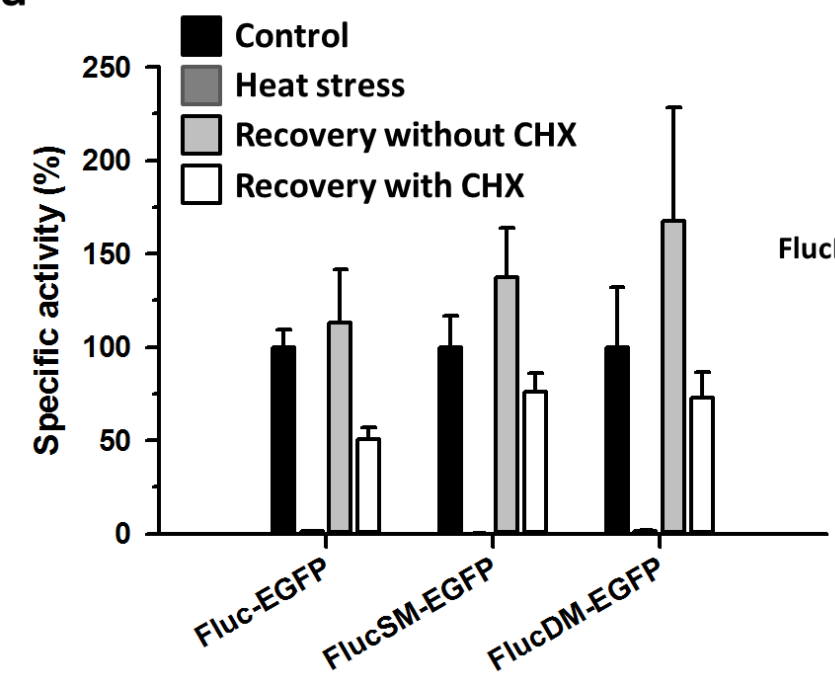

b

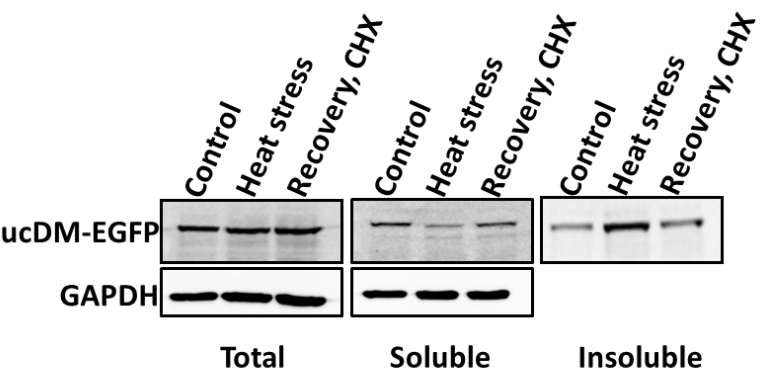

Figure 26: Assessment of refolding capacity of HeLa cells using Fluc-EGFP sensors.

(a) Specific activity of EGFP-tagged Fluc sensor proteins in HeLa cells upon heat stress $\left(2 \mathrm{~h}\right.$ at $\left.43^{\circ} \mathrm{C}\right)$ and recovery $\left(2 \mathrm{~h}\right.$ at $\left.37^{\circ} \mathrm{C}\right)$ in presence or absence of cycloheximide $(\mathrm{CHX})$. Specific activities in control cells maintained at $37^{\circ} \mathrm{C}$ were set to $100 \%$ in each case. Error bars indicate s.d., $\mathrm{n}=3$. (b) Cell-fractionation experiment showing total cell extract, detergent-soluble and insoluble fraction of FlucDM-EGFP expressing HeLa cells treated as in a. FlucDM-EGFP was detected by immunoblotting with an antibody against GFP.

To further validate that resolubilization of aggregates had occurred, we performed a cell fractionation experiment with FlucDM-EGFP expressing cells (Figure 26b). In this experiment, we prepared three fractions - total lysate, a detergent-soluble fraction and an insoluble (pellet) fraction from cells maintained under three different conditions; without heat stress (control), heat stress at $43^{\circ} \mathrm{C}$ for $2 \mathrm{~h}$ and recovery in the presence of $\mathrm{CHX}$ at $37^{\circ} \mathrm{C}$ for $2 \mathrm{~h}$ following heat stress. While there was no change in the band intensity of FlucDM-EGFP in total lysate of cells under the different conditions, we observed a decrease in the soluble fraction of cells exposed to heat stress. A corresponding increase in the band intensity in the insoluble fraction under same conditions suggests that aggregation of FlucDM-EGFP has occurred. A relatively high band intensity in the soluble fraction of cells that were allowed to recover, compared to heat stressed cells, indicates that resolubilization of FlucDM-EGFP aggregates has occurred. These results therefore further confirm that most of FlucDM-EGFP protein was insoluble after heat stress, but 
is efficiently resolubilized during recovery in presence of cycloheximide (Figure 26b). Hence, the Fluc-EGFP variants have allowed assessment of cellular capacity to refold heat-denatured protein.

The differential sensitivity of Fluc variants towards denaturation in RRL and in vivo shows that the proteins can function as sensors of proteostasis imbalance during stress conditions. The following sections will show the results obtained as part of the in vivo characterization of the Fluc-EGFP based sensors during various conditions that challenge cellular proteostasis.

\section{V.3.4. Effect of Fluc-EGFP-based Sensors on the Cytosolic Stress}

\section{Response}

EGFP-tagged FlucSM and FlucDM were structurally destabilized (as shown by the proteinase K assay, Figure 24) and hence they themselves may cause intrinsic stress to the cells. Ideally, to qualify as good sensor proteins, the Fluc variants should not affect the proteostasis capacity of the cellular system under investigation (or do so only minimally). To investigate this possibility, we transiently co-transfected Fluc-EGFP variants in HeLa cells with a reporter for the cytosolic stress response. The reporter consisted of the structurally unrelated Renilla reniformis luciferase (Rluc) under regulation of the stress-sensitive HSPAlA (Hsp70) promoter (Figure 27a). The rationale for using this reporter was that a stress condition such as heat shock will cause HSF1 binding to the HSPA1A promoter (Baler et al., 1993; Sarge et al., 1993; Westwood et al., 1991) leading to synthesis of Rluc and therefore an increase in its enzymatic activity during recovery. If Fluc variants also induce some stress to cells, an increase in Rluc activity is expected.

In this experiment, the co-transfection efficiency of Fluc-based sensors and HSPAlARluc construct in cells as determined by fluorescence microscopy was $\sim 70 \%$. This means that the majority of the cells express both constructs and therefore Rluc activity will mainly reflect the effect of Fluc-EGFP variants on the cytosolic stress response. As expected, heat stress treatment $\left(43^{\circ} \mathrm{C}, 2 \mathrm{~h}\right)$ followed by recovery $\left(37^{\circ} \mathrm{C}, 2 \mathrm{~h}\right)$ to cells containing only HSPAlA-Rluc reporter resulted in $\sim 10$-fold induction of Rluc activity and a corresponding increase in Rluc protein amount upon immunoblotting (Figure 27). A similar increase in Rluc activity was 
observed when cells co-expressing Fluc-EGFP variants were treated under the same conditions. This indicates that the cells expressing the sensors respond normally to heat stress and there is no additional contribution to the cytosolic stress response from the misfolded Fluc mutant proteins. In the control experiment, the expression of the Fluc-EGFP variants at $37^{\circ} \mathrm{C}$ without heat stress caused only a 1.5-2 fold induction of Rluc activity. Among the Fluc variants, Fluc-EGFP showed the most notable effect probably due to its higher expression than FlucSM-EGFP and FlucDMEGFP (Figure 27b).

a


b

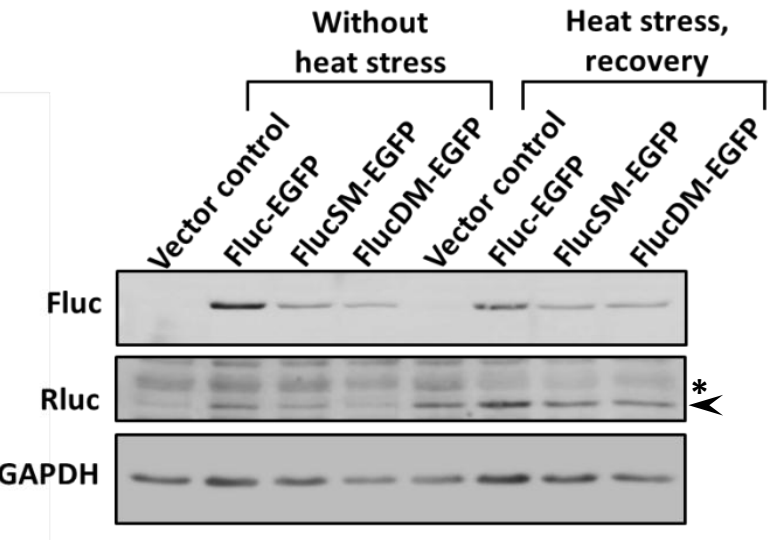

Figure 27: Effect of EGFP-tagged Fluc sensor proteins on the cytosolic stress response.

(a) HeLa cells were transiently transfected with the stress responsive HSPAIA-Rluc-myc reporter (top) along with the plasmids encoding Fluc-EGFP variants or vector-only control. Rluc activity was measured either without stress or after subjecting the cells to heat stress for $2 \mathrm{~h}$ at $43^{\circ} \mathrm{C}$ followed by recovery for $2 \mathrm{~h}$ at $37^{\circ} \mathrm{C}$. Error bars indicate s.d., $\mathrm{n}=3$. RLU stands for relative luminescence unit. (b) Amounts of stressresponsive Rluc and Fluc-EGFP variants in HeLa cells treated as in a, detected by immunoblotting. Arrow indicates Rluc band. Asterisk indicates a non-specific band. GAPDH was used as a loading control.

We further validated the results obtained from Rluc activity measurements and estimation of Rluc amounts by immunoblotting (Figure 27) by performing immunocytochemistry experiment (Figure 28). At $37^{\circ} \mathrm{C}$, the cells expressing Fluc-EGFP showed very weak Cy3 fluorescence from myc-tagged Rluc in contrast to cells expressing Fluc-EGFP mutants. This suggests that Fluc-EGFP mutants minimally induce the stress response under normal growth 
conditions, presumably due to their structural flexibility. A substantial increase in Cy3 fluorescence was observed in cells expressing Fluc-EGFP variants upon heat stress, consistent with the increased synthesis of Rluc protein. There was no detectable difference in the Cy3 fluorescence intensities of cells with or without Fluc-EGFP variants, suggesting that mutant proteins when expressed at a low-level caused only a very mild activation of the cytosolic stress response.

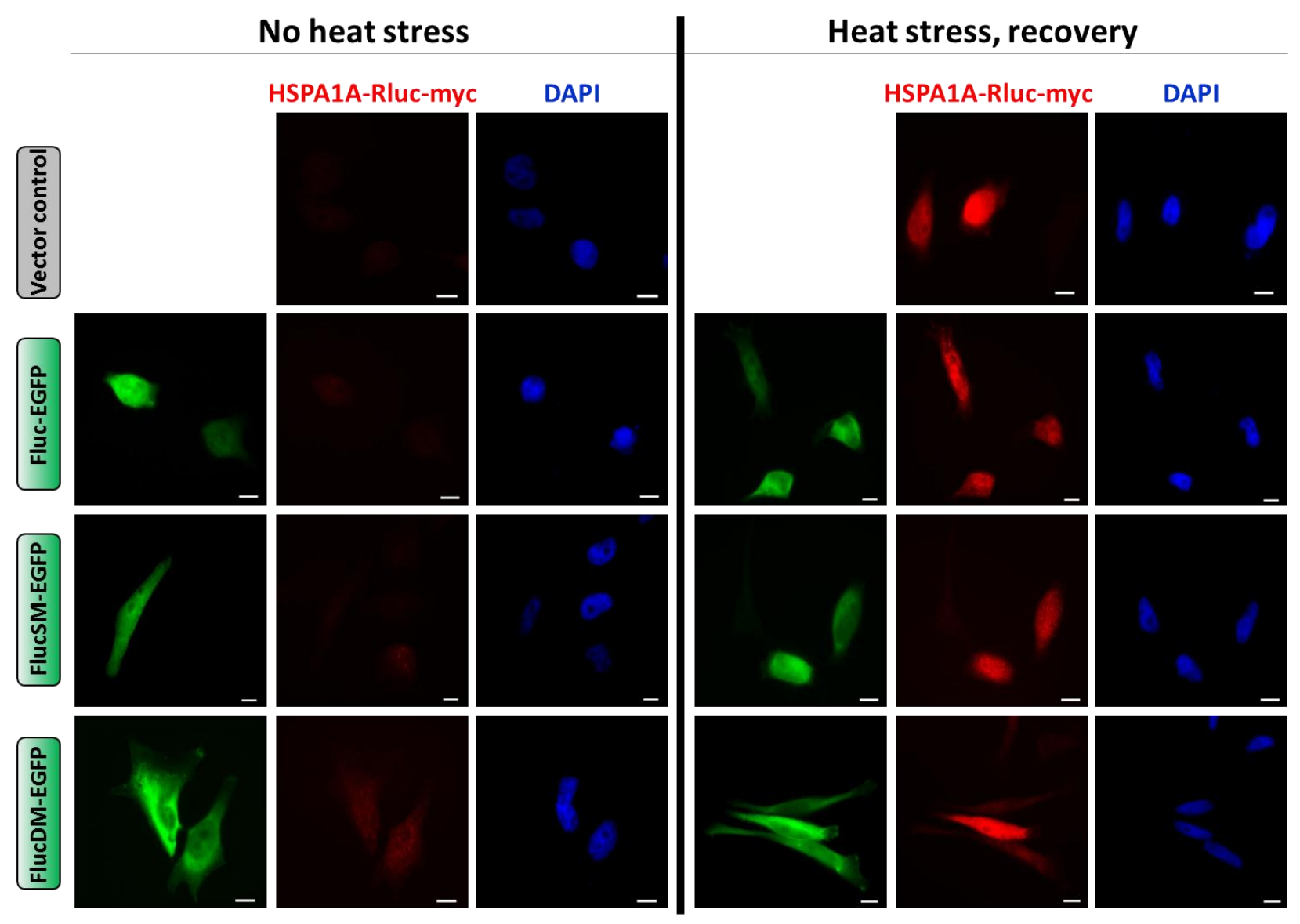

Figure 28: Fluc-EGFP based sensors minimally induce cytosolic stress response.

Representative immunofluorescence micrographs of HeLa cells co-expressing HSPAlA-Rluc-myc and Fluc-EGFP variants. The cells were either maintained at $37^{\circ} \mathrm{C}$ (no heat stress) or subjected to heat stress for $2 \mathrm{~h}$ at $43^{\circ} \mathrm{C}$ followed by recovery for $2 \mathrm{~h}$ at $37^{\circ} \mathrm{C}$. Rluc was detected by immunocytochemistry against the Myc tag with anti-Myc primary antibody followed by $\mathrm{Cy} 3$ conjugated secondary antibody (red). FlucEGFP variants were detected by GFP fluorescence and nuclei were stained with DAPI (blue). Scale bars correspond to $10 \mu \mathrm{m}$. 


\section{V.4. Applications of Fluc-EGFP-based Sensors in vivo}

\section{V.4.1. Assessment of Proteostasis Capacity in Presence of Small Molecule Inhibitors}

As discussed in section II.4.5, many small molecule compounds are known to target different components of the proteostasis network and therefore can be used to modulate the proteostasis capacity of cells. Since folding and degradation machineries form central hubs of the proteostasis network in cells (Gidalevitz et al., 2010), we used Fluc variants to measure imbalances in proteostasis after alteration of these two essential pathways. We modulated the folding pathway by inhibiting Hsp90 while degradation was inhibited by targeting the UPS. To test whether the Fluc-EGFP based sensors can report such changes, we treated HeLa cells transiently expressing Fluc-EGFP variants with the Hsp90 inhibitor 17-allylamino-17demethoxygeldnamycin (17-AAG) or with the proteasome inhibitor MG132 (Figure 29).

17-AAG is a geldanamycin analog that inhibits Hsp90 by displacing ATP from its ATP binding pocket and thereby functionally arrests the chaperone cycle of Hsp90 (Sharp and Workman, 2006; Whitesell and Lindquist, 2005). Hsp90 is a major cytosolic chaperone and has been shown to refold Fluc upon recovery from heat stress (Schneider et al., 1996). Treatment of HeLa cells with $0.5 \mu \mathrm{M}$ 17-AAG for $8 \mathrm{~h}$ led to the formation of small green-fluorescent aggregates in $\sim 8 \%$ of cells expressing FlucSM-EGFP and multiple larger aggregates in $\sim 50 \%$ of cells expressing FlucDM-EGFP (Figure 29a). Fluc-EGFP expressing cells, in contrast, showed diffuse cytosolic distribution of fluorescence upon 17-AAG treatment. To precisely quantitate the effect of 17-AAG mediated Hsp90 inhibition on the proteostasis network, we measured the specific activity of Fluc-variants. Upon Hsp90 inhibition, there was a decrease in the specific activity of Fluc-EGFP by $\sim 40 \%$, of FlucSM-EGFP by $\sim 60 \%$ and of FlucDM-EGFP by $\sim 80 \%$ (Figure 29b-c). These results suggest that inhibition of Hsp90 compromise the proteostasis system, resulting in aggregation of the mutant Fluc-EGFP sensors. 
a
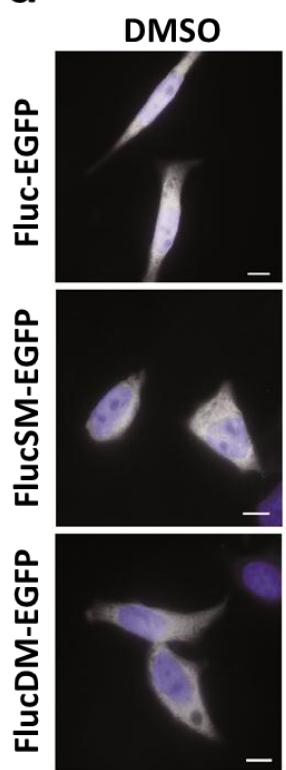

17-AAG
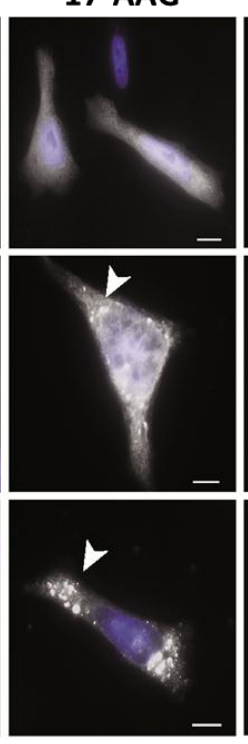

b

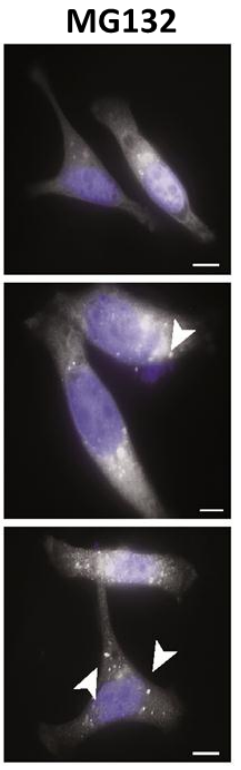

C
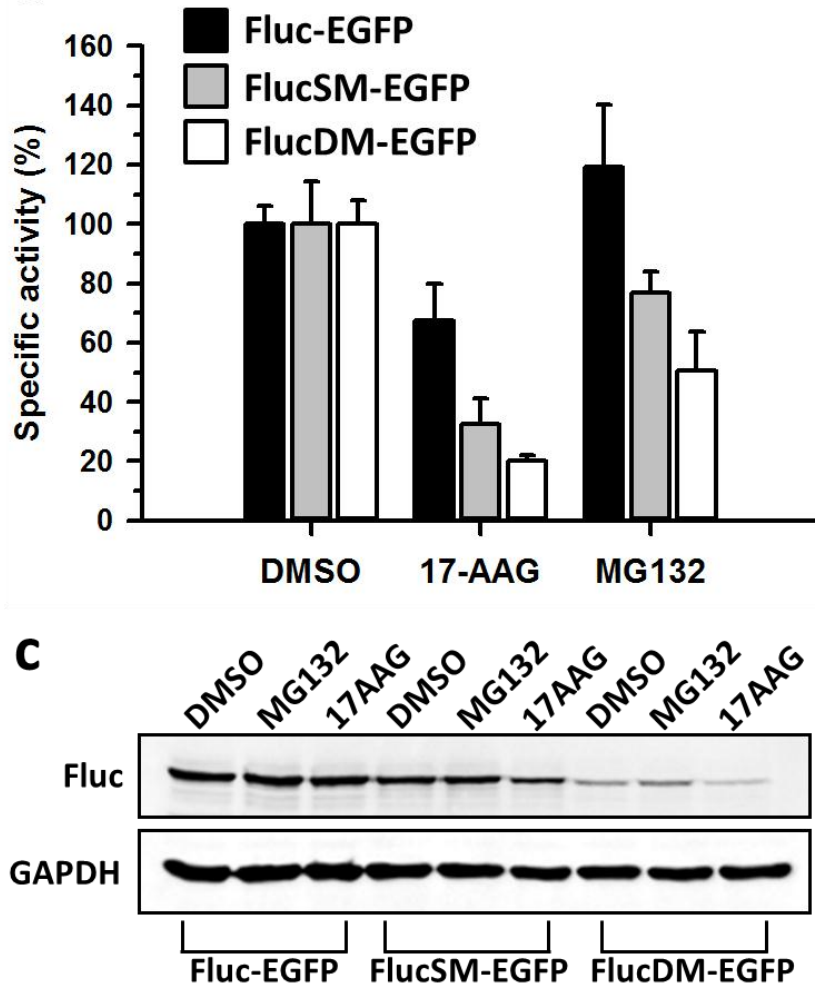

Figure 29: Fluc-mutant proteins report on impairment of proteostasis by small-molecule inhibitors.

(a) Representative fluorescence micrographs of HeLa cells expressing Fluc-EGFP, FlucSM-EGFP or FlucDM-EGFP treated with $0.1 \%$ DMSO, $0.5 \mu \mathrm{M}$ 17-AAG or $5 \mu \mathrm{M}$ MG132 for $8 \mathrm{~h}$. EGFP fluorescence is shown in white. Arrowheads indicate aggregates. Nuclei were stained with DAPI (blue). Scale bars correspond to $10 \mu \mathrm{m}$. (b) Specific activities of Fluc-EGFP variants in HeLa cells treated as above. DMSO control values were set to $100 \%$. Error bars indicate s.d., $n=3$. (c) A representative immunoblot showing the amounts of Fluc-EGFP variants in HeLa cells treated as in a, detected by anti-GFP antibody. GAPDH was used as a loading control.

MG132 is a small molecule that reversibly inhibits the chymotrypsin-like activity of the proteasome and hence leads to accumulation of polyubiquitinated proteins in cells which causes proteome stress (Kisselev and Goldberg, 2001; Myung et al., 2001). We measured the effect of proteasome inhibition on Fluc-based sensors by fluorescence microscopy of HeLa cells that were treated with $5 \mu \mathrm{M}$ MG132 for $8 \mathrm{~h}$. Here, we observed small aggregate-like structures of FlucEGFP in $\sim 25 \%$ of cells expressing Fluc-EGFP, whereas $~ 70 \%$ of FlucSM-EGFP expressing cells and $\sim 90 \%$ of FlucDM-EGFP expressing cells contained aggregates (Figure 29a). A possible explanation for this might be the accumulation of Fluc-EGFP mutant proteins upon proteasome inhibition. Therefore aggregation might have resulted from an increase in their 
cellular concentrations. However, MG132 treatment led only to a slight increase in the total cellular amount of these proteins as detected by immunoblotting (Figure 29c), which suggests that proteasome inhibition doesn't have a direct influence on the stability of Fluc-EGFP variants and therefore the aggregation of these sensor proteins could be caused by a general proteostasis imbalance. We further followed the effect of proteasome inhibition by MG132 on Fluc-EGFP variants by measuring the specific activity of the proteins. Upon proteasome inhibition, there was a minor effect on the specific activity of Fluc-EGFP but the specific activities of FlucSM-EGFP and FlucDM-EGFP were reduced by $\sim 20 \%$ and $\sim 50 \%$, respectively (Figure 29b). Thus, Fluc mutants are highly sensitive in reporting the impairment of proteostasis capacity from Hsp90 or proteasome inhibition and can be used to measure the changes in folding and degradation capacity of cells.

\section{V.4.2. Analysis of Proteostasis Collapse by Huntingtin Protein}

As mentioned in section II.4.3.1, Huntington's disease (HD) is a progressive neurodegenerative disorder caused by expansion of a polyglutamine (polyQ) sequence in the huntingtin (Htt) protein. Studies have shown that expansion of polyQ sequences beyond 35-40 residues results in the formation of aggregates in the nucleus (Davies et al., 1997; DiFiglia et al., 1997) and leads to cellular toxicity. Moreover, expression of polyQ-containing proteins in the nematode $C$. elegans has been shown to compromise the cellular folding capacity and to disrupt proteostasis (Gidalevitz et al., 2006).

To investigate the sensitivity of Fluc-based sensors to changes in proteostasis in presence of Htt-polyQ, we used HEK 293T cells (cells derived from human embryonic kidney and transformed with the T-antigen). It is known that expansion of polyQ stretch in the exon1 of the Htt gene beyond 35 residues results in Htt aggregation (Davies et al., 1997; DiFiglia et al., 1997). Therefore, we transiently co-transfected cells with plasmids encoding the Fluc-EGFP variants and with $m$ Cherry-tagged $H t t$ exon1 constructs encoding proteins with normal (20-glutamine; 20Q) or expanded (97-glutamine; 97Q) polyQ sequences. We observed large polyQ-containing aggregates $48 \mathrm{~h}$ after transfection in almost all cells expressing Htt-97Q, whereas Htt-20Q remained diffusely distributed (Figure 30). When we expressed Fluc-EGFP together with Htt97Q, small aggregate foci of Fluc-EGFP were observed in only $20 \%$ of the cells. However, multiple and more distinct aggregates were detected in 50\% of cells co-expressing FlucSM- 
EGFP and Htt-97Q and massive aggregation of FlucDM-EGFP was observed in 85\% of cells coexpressing FlucDM-EGFP and Htt-97Q (Figure 30). The aggregates of Fluc-EGFP mutant proteins varied in their size and localization. In cells expressing Htt-20Q and Fluc-EGFP variants, we detected no aggregates. Thus, the sensors confirmed findings in $C$. elegans that expression of polyQ expansion proteins results in a severe decline of cellular proteostasis and disturbs the folding of metastable proteins (Gidalevitz et al., 2006).
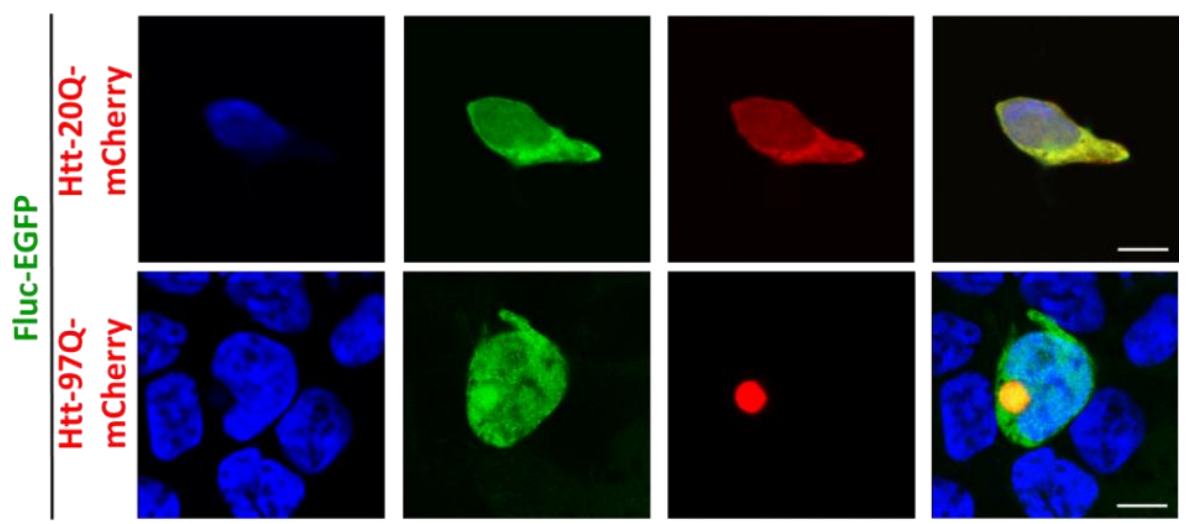

Figure 30: Flucbased sensors report on proteostasis impairment by mutant Htt.


Representative

fluorescence confocal micrographs of HEK $293 \mathrm{~T}$ cells $48 \mathrm{~h}$ after cotransfection with vectors encoding FlucEGFP variants and mCherry-tagged $\mathrm{Htt}$ proteins with 20Q or 97Q. EGFP and mCherry fluorescence are shown in green and
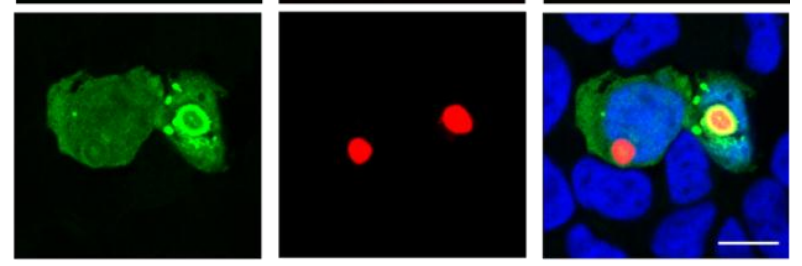
red respectively. Nuclei were stained with DAPI (blue). Scale bars correspond to $10 \mu \mathrm{m}$.

To obtain further insight into the process of proteostasis collapse by polyQ expansion proteins and to follow the aggregation kinetics of Fluc-based sensors in presence of Htt-97Q, we performed live-cell imaging of HEK 293T cells. The live-cell imaging technique allows real time monitoring of cellular events by acquiring images of cells after specified regular intervals for a fixed time period. Therefore, real-time changes in the folding status of aggregation prone proteins can be easily followed. We imaged cells expressing either the stable Fluc-EGFP or the least stable FlucDM-EGFP together with mCherry tagged Htt-97Q 24 h after transfection for a period of $44 \mathrm{~h}$ (Figure 31$)$.

In cells expressing Fluc-EGFP and Htt-97Q, we observed the aggregates of Htt-97Q after $27 \mathrm{~h}$ of transfection whereas no aggregates of Fluc-EGFP were detected until $44 \mathrm{~h}$ (Figure 31a). In cells expressing FlucDM-EGFP and Htt-97Q, the aggregation of FlucDM-EGFP started with the formation of small, multiple aggregate foci (at $26 \mathrm{~h}$ ) that gradually increased in size during the course of Htt-97Q aggregation (Figure 31b). The formation of FlucDM-EGFP aggregates preceded the aggregation of Htt-97Q. FlucDM-EGFP began to aggregate after $26 \mathrm{~h}$ compared to Htt-97Q which showed aggregates around $2 \mathrm{~h}$ later. This indicates that collapse of proteostasis might have occurred before the appearance of detectable Htt-97Q aggregates. This observation is in line with other studies that suggest a role of the oligomeric species of the mutant Htt protein in proteostasis imbalance and cellular toxicity (Arrasate et al., 2004; Bodner et al., 2006; Miller et al., 2010; Saudou et al., 1998; Takahashi et al., 2008). However, after the mutant Htt formed visible aggregates, the aggregation of FlucDM-EGFP became more severe and rapid which may suggest that aggregation of Htt continues to impose a challenge on the proteostasis machinery of cells. In control experiment, cells co-expressing FlucDM-EGFP and Htt-20Q mCherry did not show aggregation of either protein (Figure 31c). Thus, while Fluc-EGFP remains stable, FlucDM-EGFP exquisitely responds to the decline in proteostasis capacity and FlucDM-EGFP aggregation serves as a marker for proteostasis imbalance even before Htt inclusions are visible. 
a

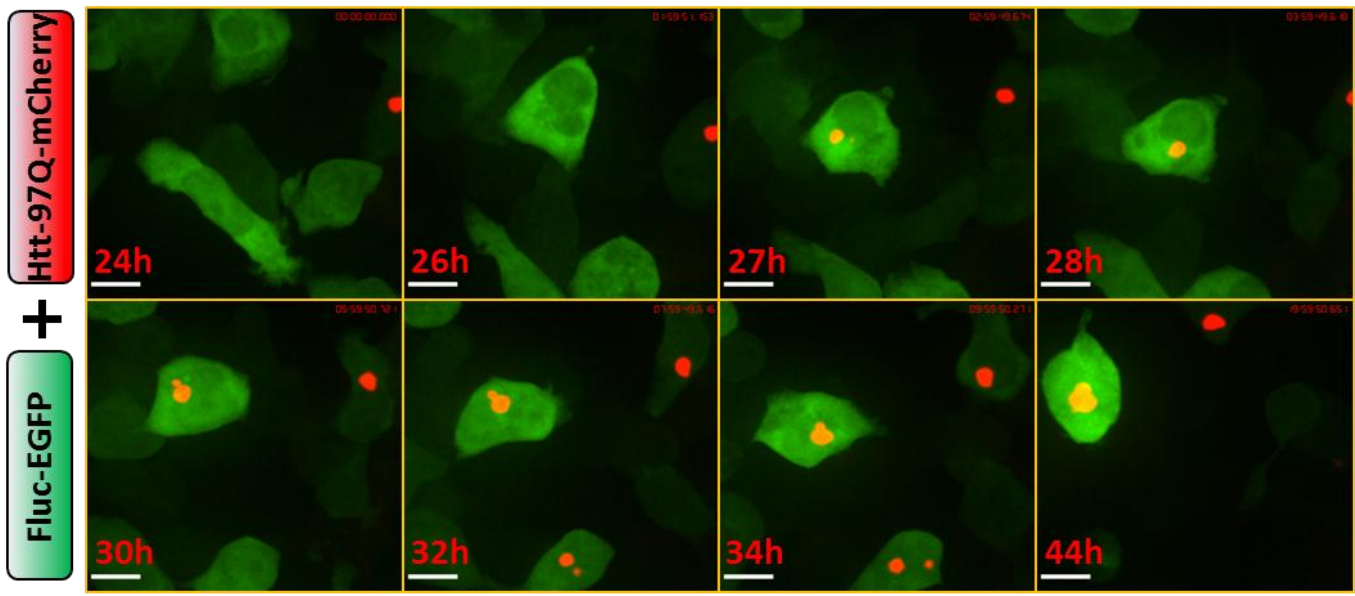

b



C



Figure 31: Time-course analysis of proteostasis collapse induced by mutant Htt.

Representative confocal micrographs from live-cell imaging of HEK 293T cells co-expressing (a) FlucEGFP and Htt-97Q-mCherry, (b) FlucDM-EGFP and Htt-97Q-mCherry or (c) FlucDM-EGFP and Htt20Q-mCherry at the times indicated. Image acquisition started $24 \mathrm{~h}$ after co-transfection of HEK 293T cells with vectors encoding Fluc-EGFP variants and Htt-20Q or 97Q-mCherry. EGFP and mCherry fluorescence are shown in green and red respectively. Scale bars correspond to $7 \mu \mathrm{m}$. 


\section{V.4.3. Assessing Proteostasis in C. elegans Upon Heat Stress}

Having demonstrated the sensitivity of Fluc-mutants in vitro and in a cell culture model system, we tested their applications as proteostasis sensors in an entire organism. We chose the multi-cellular nematode $C$. elegans as a model organism since it is simple and easy to grow and to manipulate genetically. Adult $C$. elegans (hermaphrodite) contains 959 somatic cells, out of which 302 are neuronal cells and 95 are body wall muscle cells (Sulston and Horvitz, 1977). Moreover, it has a short life span of 2-3 weeks and a generation time of 3-4 days which confers an additional advantage to study cellular differentiation and aging processes. C. elegans has been used previously to study changes in proteostasis during different conditions such as heat stress or in presence of mutant Htt (Gidalevitz et al., 2011). To check the sensitivity of FlucEGFP variants in C. elegans as a tool to monitor proteome stress, the proteins were expressed in body-wall muscle cells using the unc-54 promoter or in neuronal cells using the $F 25 B 3.3$ promoter as previously described (Morley and Morimoto, 2004) (Figure 32).
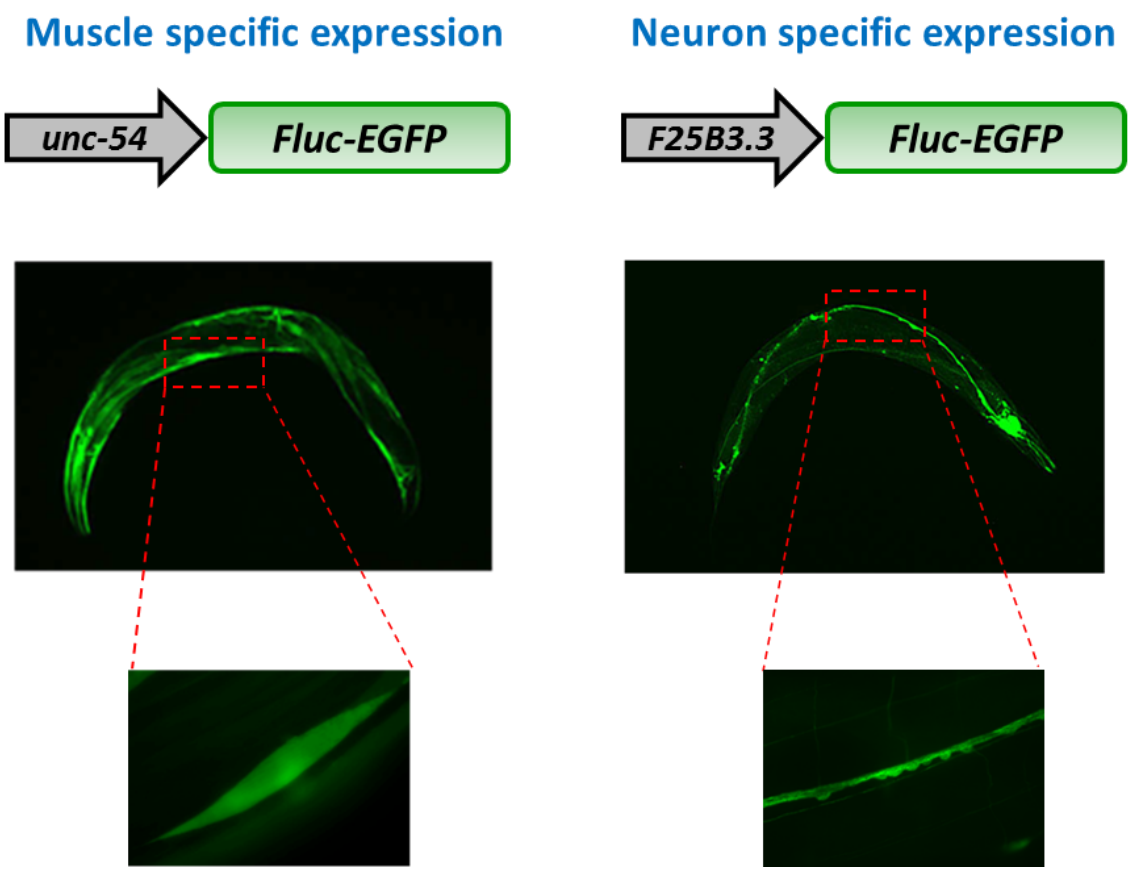

Figure 32: $C$. elegans transgenic lines expressing Fluc-based sensors in body-wall muscle cells or neuronal cells.

Representative fluorescence micrographs of $C$. elegans showing the expression of Fluc-EGFP either under muscle specific promoter (unc-54) or under neuron specific promoter (F25B3.3). A section of muscle tissue or neuron tissue is enlarged for better clarity. EGFP fluorescence is shown in green. 
Under normal growth conditions at $20^{\circ} \mathrm{C}$, the young adult worms (day 1) expressing FlucEGFP variants in body-wall muscle cells showed diffuse distribution of the sensor proteins (Figure 33a). However, upon heat stress at $33^{\circ} \mathrm{C}$ for $1 \mathrm{~h}$ (Morley and Morimoto, 2004), FlucDMEGFP formed aggregates of various sizes that were dispersed throughout the muscle cells, whereas Fluc-EGFP and FlucSM-EGFP remained diffusely distributed. Upon recovery (from heat stress), by incubating worms at $20^{\circ} \mathrm{C}$ for $6 \mathrm{~h}$, the number and size of aggregates of FlucDMEGFP decreased. A recovery of $24 \mathrm{~h}$ led to complete disappearance of aggregates in worms expressing FlucDM-EGFP and diffusely distributed EGFP fluorescence predominated, suggesting that the $C$. elegans body-wall muscle cells are able to either resolubilize or degrade the aggregates (Figure 33a).

a

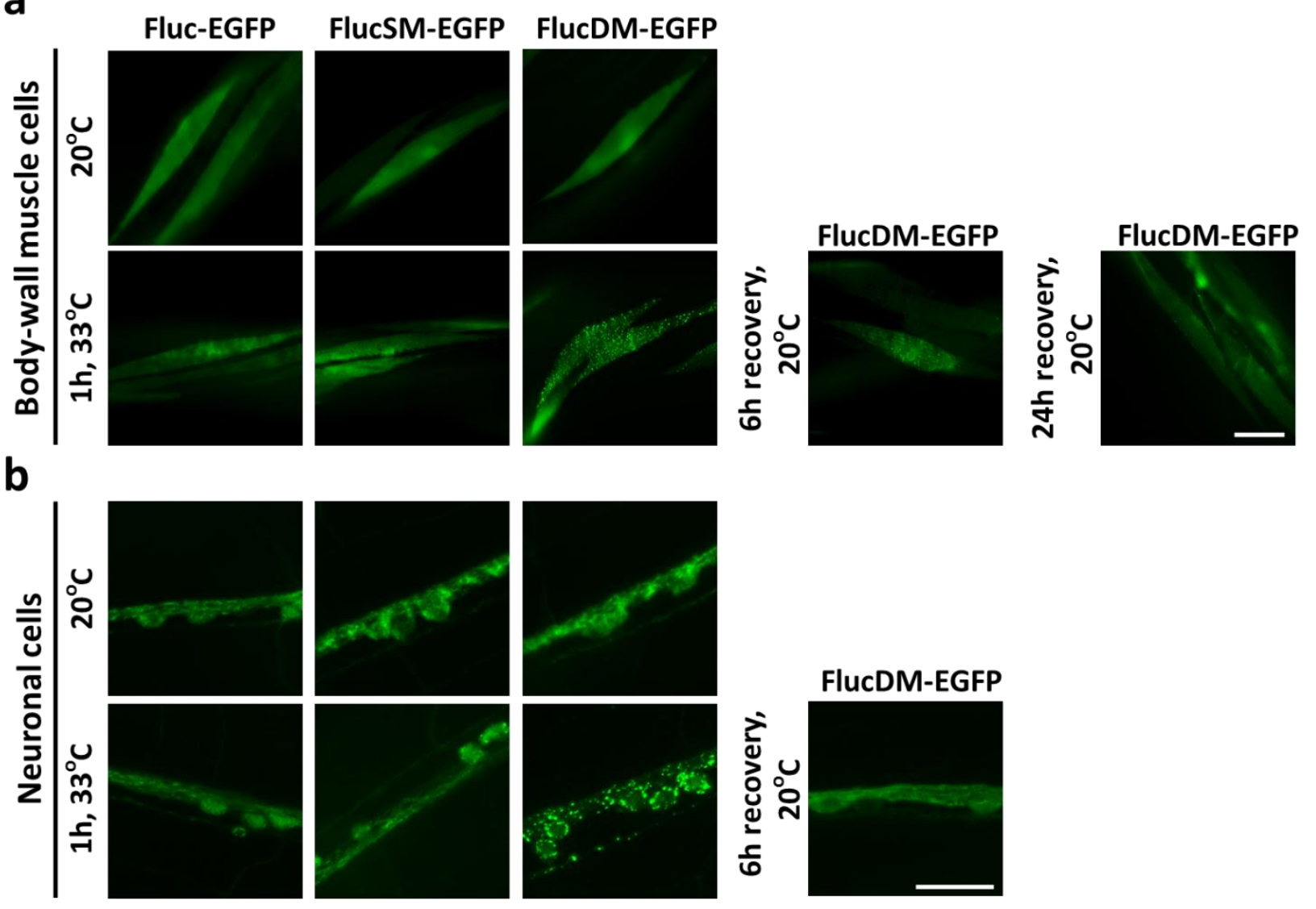

Figure 33: Fluc-based sensors report on acute proteome stress during heat shock in $C$. elegans.

(a,b) Representative fluorescence micrographs of young-adult worms (day 1) expressing Fluc-EGFP variants in body-wall muscle cells (a) or neuronal cells (b) under normal growth conditions at $20^{\circ} \mathrm{C}$, after heat stress for $1 \mathrm{~h}$ at $33^{\circ} \mathrm{C}$ and after recovery at $20^{\circ} \mathrm{C}$ for $6 \mathrm{~h}$ and $24 \mathrm{~h}$ (muscle specific expression) or $6 \mathrm{~h}$ (neuron specific expression). EGFP fluorescence is shown in green. Scale bars correspond to $10 \mu \mathrm{m}$. 
We obtained similar results from worms expressing the sensor proteins in neuronal cells (Figure 33b). Under normal growth conditions, the Fluc-EGFP variants were diffusely distributed in neuronal cells. Upon heat stress, FlucDM-EGFP formed aggregates, whereas FlucEGFP and FlucSM-EGFP remained soluble. Upon recovery for $6 \mathrm{~h}$, the aggregates of FlucDMEGFP disappeared completely in neuronal cells.

The tissue-specific differences in the recovery of FlucDM-EGFP aggregates could possibly have resulted from differential expression of sensor protein in neuronal and muscle cells. We controlled for this possibility by performing semi-quantitative reverse-transcriptasePCR (RT-PCR). Here, we monitor the relative mRNA level of FlucDM-EGFP with respect to constitutively expressed muscle-specific endogenous protein, unc-54 or neuron-specific endogenous protein, unc-119 (Figure 34). We found that the fold change in mRNA level of FlucDM-EGFP relative to unc-54 was similar in comparison to its fold change relative to unc119. This indicates that tissue-specific differences in the recovery of FlucDM-EGFP aggregates were not due to the differential expression of sensor protein in neuronal and muscle cells. Therefore, the faster recovery of aggregated FlucDM-EGFP in neuronal cells $(6 \mathrm{~h})$ compared to muscle cells $(24 \mathrm{~h})$ suggests that in young-adult worms neuronal cells are more efficient in responding to proteostasis imbalance than muscle cells.

a

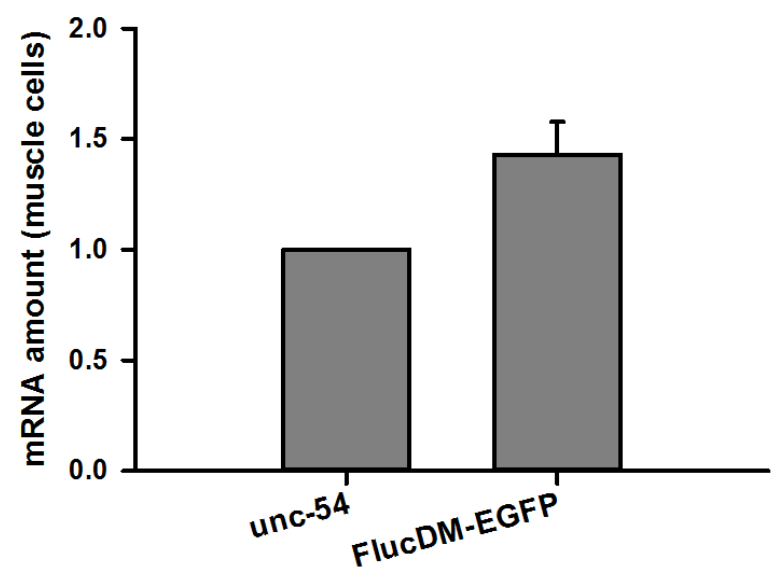

b

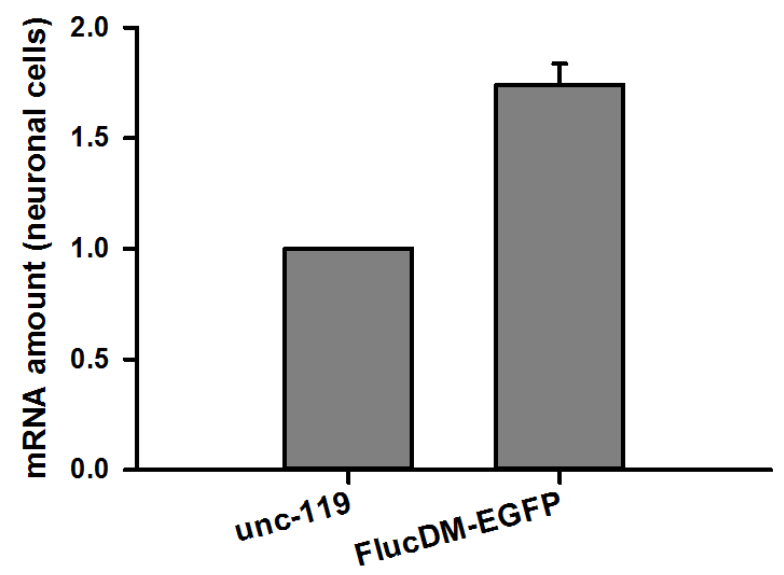

Figure 34: Tissue-specific expression level of FlucDM-EGFP in $C$. elegans.

Bar graphs showing the relative mRNA amounts of FlucDM-EGFP in (a) body-wall muscle cells and in (b) neuronal cells, determined by RT-PCR. unc-54 (muscle-specific) and unc-119 (neuron-specific) served as endogenous controls. Error bars indicate s.d., $n=3$. 


\section{V.4.4. Assessing Proteostasis in C. elegans During Aging}

In $C$. elegans, a progressive decline of proteostasis capacity during aging has been demonstrated (Ben-Zvi et al., 2009). To test the ability of the Fluc-based sensors to measure the changes in the cellular proteostasis during aging, we analyzed the distribution patterns of the Fluc-EGFP variants in muscle and neuronal cells of transgenic $C$. elegans throughout their adult lifespan (Figure 35).

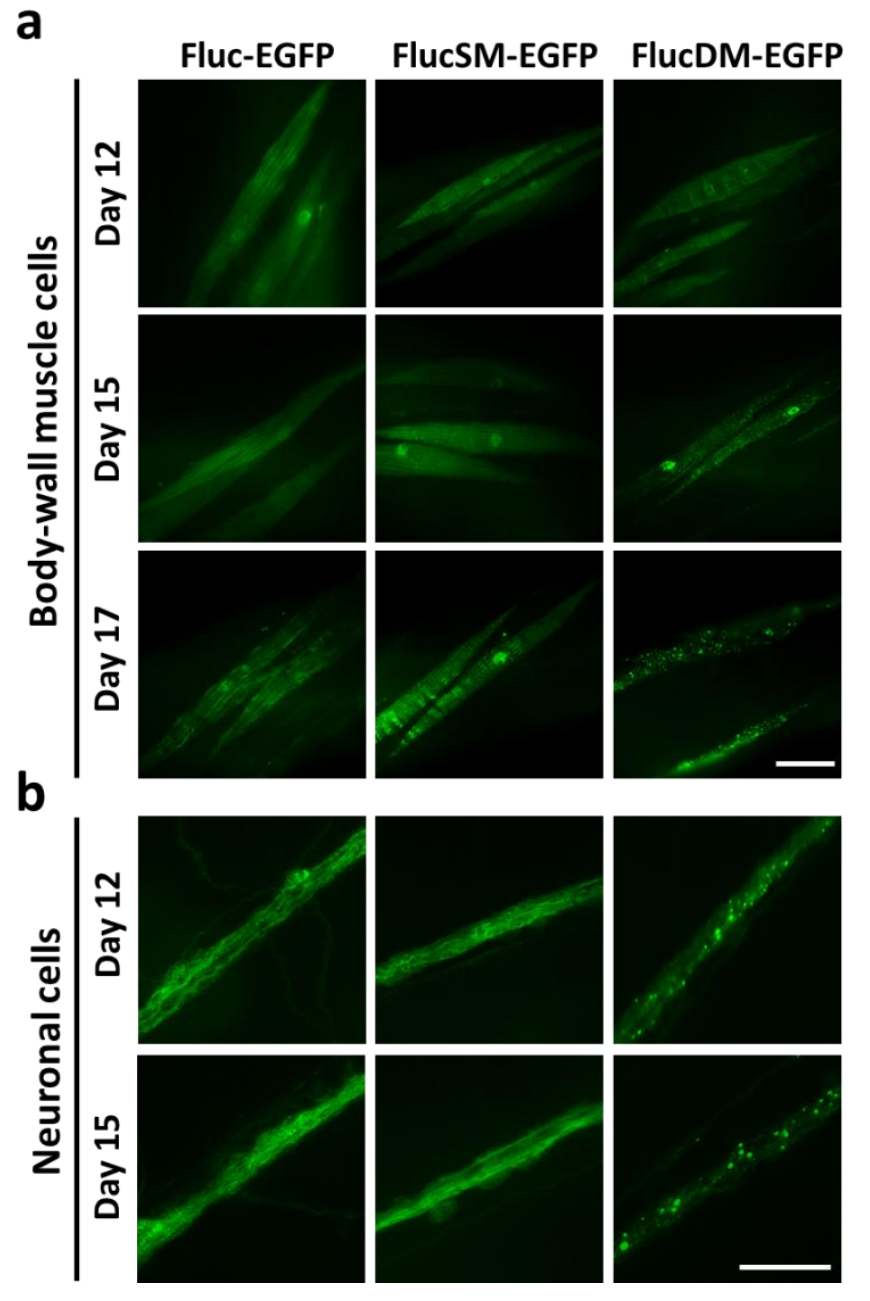

C

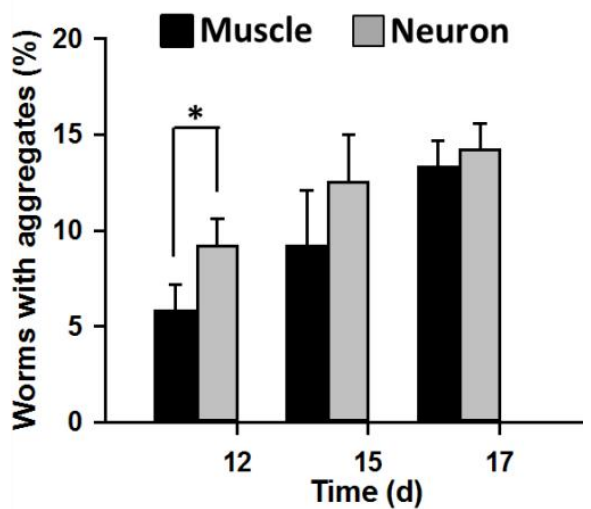

Figure 35: Fluc-based sensors report on proteostasis decline during aging in C. elegans.

(a,b) Representative fluorescence micrographs of worms expressing FlucEGFP variants in body-wall muscle cells (a) or in neuronal cells (b) under normal growth conditions at $20^{\circ} \mathrm{C}$ imaged on indicated days. EGFP fluorescence is shown in green. Scale bars correspond to $10 \mu \mathrm{m}$. (c) Percentage of FlucDM-EGFPexpressing worms containing aggregates on indicated days. Error bars indicate s.d., $\mathrm{n}=3$. Forty worms were counted in each experiment. ${ }^{*} \mathrm{P}<0.05$ (Student's paired $t$ test).

The worms expressing Fluc-EGFP and FlucSM-EGFP in body-wall muscle cells showed diffuse distribution until day 17, but those expressing FlucDM-EGFP displayed small aggregates from day 12 on (Figure 35a). The aggregates of FlucDM-EGFP increased both in number and size until day 17, with $\sim 15 \%$ of the worms containing distinct aggregates (Figure 35a, c). Similarly, in neuronal cells Fluc-EGFP and FlucSM-EGFP maintained their diffuse distribution 
while FlucDM-EGFP tended to aggregate more frequently already at days 12 and 15 (Figure 35b). Thus, in young-adult worms, neuronal cells are more efficient in recovering from acute stress (such as heat stress) than muscle cells (Figure 34); however, they appeared to be less capable of maintaining proteostasis during chronic proteome stress during aging (Figure 35).

\section{V.4.5. Analysis of the Proteostasis Capacity of Cytosol and Nucleus V.4.5.1. Design and Biochemical Characterization of NLS- and NES-Fluc- EGFP Variants}

The Fluc-EGFP variants characterized so far in vivo, although localized predominantly in cytosol, were also present in nucleus under normal conditions at $37^{\circ} \mathrm{C}$. Therefore, these mutant proteins are most likely reporting the global status of proteostasis in cells under different stress conditions. However, to measure and compare subtle changes in proteostasis in specific cellular compartments such as the cytosol and the nucleus, we created sensor proteins that localize exclusively in these compartments. To do this, we tagged Fluc-EGFP and FlucDM-EGFP with either a nuclear localization signal (NLS) or a nuclear export signal (NES) at their N-terminus to target them to the nucleus or to retain them in the cytosol (Figure 36).

\section{Fluc-EGFP}

FlucDM-EGFP
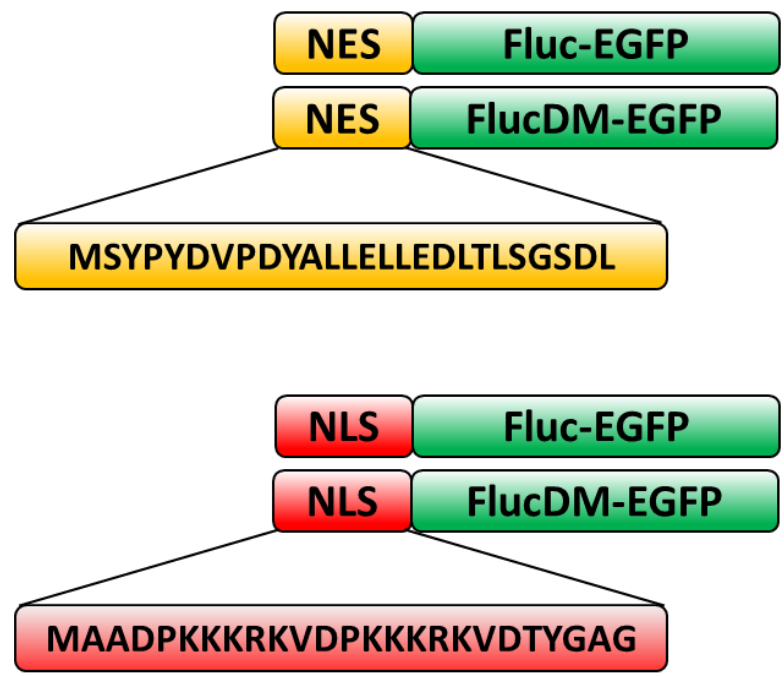

Figure 36: Design of Fluc-based sensors to study proteostasis in the cytosol and the nucleus.

The constructs Fluc-EGFP and FlucDM-EGFP without any signal sequence were used to study overall cellular proteostasis. Fluc-EGFP and FlucDM-EGFP were tagged with either a nuclear export signal (NES) (shown in yellow) or a nuclear localization signal (NLS) (shown in red) at the $\mathrm{N}$-terminus to study proteostasis of the cytoplasm and the nucleus respectively.

The NLS sequence was derived from the SV40 large T antigen and contains two repeats of a positively charged stretch rich in lysine residues (Fischer-Fantuzzi and Vesco, 1988) 
whereas, NES was derived from a consensus sequence obtained from prediction algorithm program (The NES predictor, NetNES) (http://www.cbs.dtu.dk/services/NetNES/) using experimentally validated sequences rich in leucine residues (la Cour et al., 2004).
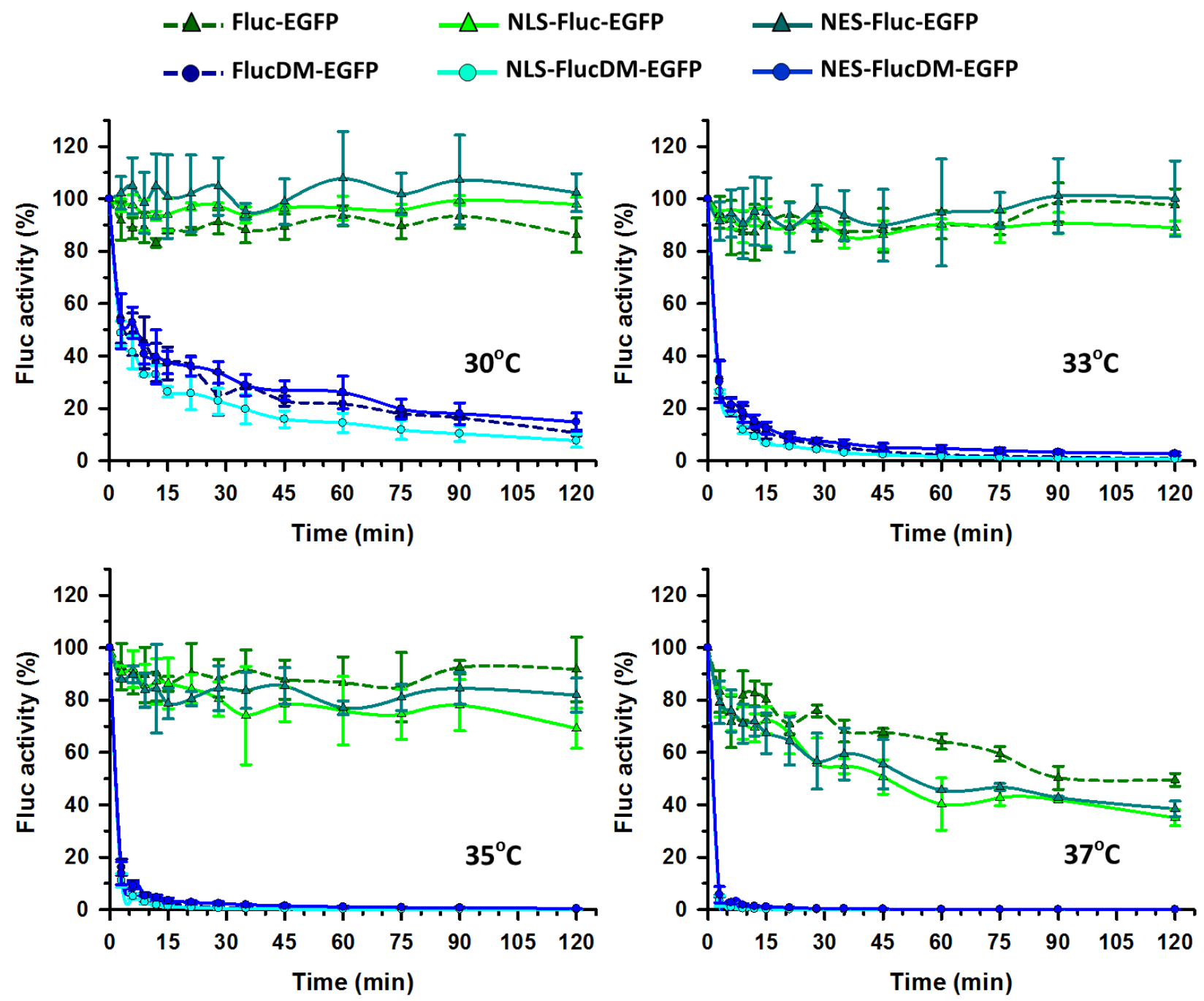

Figure 37: Temperature-dependent denaturation of NLS- or NES-Fluc-EGFP variants in RRL.

NLS-Fluc-EGFP variants, NES-Fluc-EGFP variants and Fluc-EGFP variants (without signal sequence) were translated in rabbit reticulocyte lysate at $30^{\circ} \mathrm{C}$ for $90 \mathrm{~min}$, followed by inhibition of translation and incubation at $30^{\circ} \mathrm{C}$ to $37^{\circ} \mathrm{C}$. Fluc activity was measured at the times indicated and expressed in percentage of the activity measured immediately after translation at $30^{\circ} \mathrm{C}$ (set to $100 \%$ ). Error bars indicate s.d., $\mathrm{n}=3$.

To test the effect of the NLS or NES sequence on the intrinsic heat sensitivity of FlucEGFP based sensors, we used the RRL system. We synthesized the NLS- and NES-Fluc-EGFP 
variants, along with their counterparts without signal sequence in RRL at $30^{\circ} \mathrm{C}$ for 90 min and incubated them at different temperatures to measure their stability (Figure 37). Both NLS- and NES-Fluc-EGFP variants showed the same denaturation kinetics as Fluc-EGFP variants without the signal sequence. This suggests that the NLS and NES sequences per se do not alter the sensitivity of Fluc proteins to thermal denaturation in vitro.

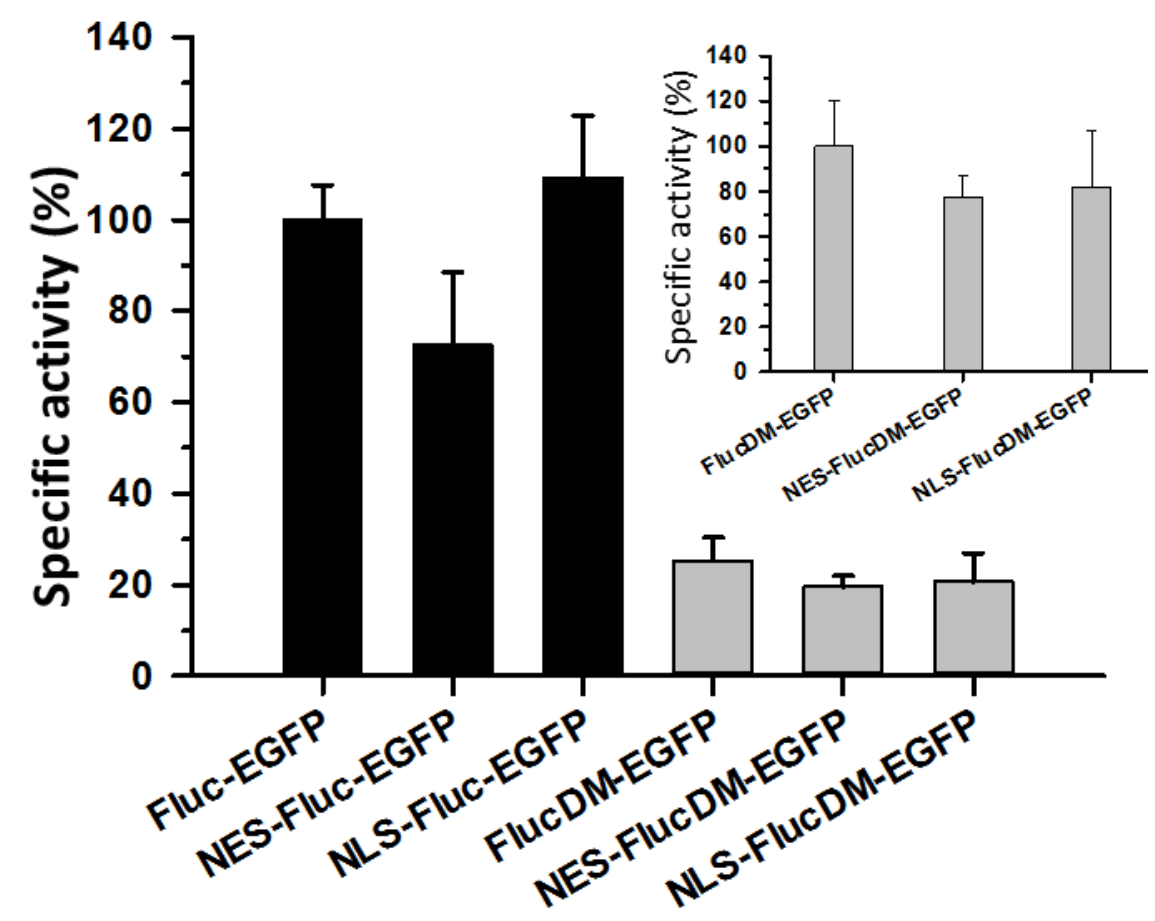

Figure 38: Biochemical characterization of compartment-specific Fluc-EGFP variants in vivo.

Fluc-EGFP variants without signal sequence and with NLS- or NES sequences were transiently expressed in HEK $293 \mathrm{~T}$ cells for $24 \mathrm{~h}$ at $37^{\circ} \mathrm{C}$. Specific activities of all the indicated Fluc-EGFP variants in soluble extracts were normalized to Fluc-EGFP without signal sequence (set to $100 \%$ ). Inset shows the specific activities of FlucDM-EGFP variants, normalized to FlucDM-EGFP without signal sequence (set to $100 \%$ ). Error bars indicate s.d., $n=3$.

We also checked the effect of the NLS and NES sequences on the stability of the FlucEGFP variants in vivo by transiently transfecting HEK 293 T cells with the constructs of FlucEGFP variants without signal sequence and with those containing an NLS- or NES signal sequence. We then measured the specific activities of the corresponding Fluc-EGFP variants (Figure 38). We observed no dramatic change in the specific activity of NLS- and NES-FlucEGFP variants compared to their counterparts without the signal sequence (Figure 38), 
supporting the results from our in vitro study which indicated that the NLS and NES sequences do not affect the enzymatic activities of the Fluc-EGFP variants in cells. This result also indicates that the proteostasis capacity of the cytosol is similar to that of the nucleus under normal conditions. Moreover, the experiment also enabled us to compare the specific activity of the Fluc-EGFP variants with the FlucDM-EGFP variants in HEK 293T cells. The specific activities of FlucDM-EGFP were 30\% of Fluc-EGFP variants, suggesting that a significant fraction of the mutant protein was misfolded in cells (Figure 38).


Figure 39: Sorting of Fluc-EGFP-based sensors to cytosol and nucleus of HEK 293T cells. Representative confocal micrographs of HEK 293T cells stably expressing cytosol localized NES-FlucEGFP and NES-FlucDM-EGFP and nucleus localized NLS-Fluc-EGFP and NLS-FlucDM-EGFP. Also shown are cells with uniformly distributed Fluc-EGFP and FlucDM-EGFP without a signal sequence. EGFP fluorescence is shown in green. Nuclei were stained with DAPI (blue). Scale bar, $10 \mu \mathrm{m}$.

To further obtain information about the inter-compartmental differences in proteostasis capacity between the nucleus and the cytosol under stress conditions, we generated stable cell lines. We transfected HEK $293 \mathrm{~T}$ cells with constructs encoding NLS- or NES-Fluc-EGFP variants and selected clones which have stably integrated NLS- or NES-Fluc-EGFP variants in their genome. We performed all further experiments with HEK 293T cells showing stable expression of nuclear or cytosolic Fluc-EGFP variants. Under normal growth conditions at $37^{\circ} \mathrm{C}$, we examined the subcellular distribution of NLS- or NES-Fluc-EGFP variants by confocal 
microscopy (Figure 39). Cells expressing NLS-Fluc-EGFP and NLS-FlucDM-EGFP showed distribution of EGFP fluorescence predominantly in the nucleus. By contrast, NES-Fluc-EGFP variants were predominantly localized in the cytosol. Fluc-EGFP variants without signal sequence were distributed both in the cytosol and the nucleus. These observations indicate that NLS- or NES-Fluc based sensors are selectively targeted to the nucleus or cytosol, respectively and thus can be used to measure inter-compartmental proteostasis.

\section{V.4.5.2. Effect of Proteasome Inhibition on the Stability of Compartment Specific Fluc Sensors}

The effect of proteasome inhibition on the proteostasis network of HeLa cells has been demonstrated earlier using untagged Fluc-EGFP based sensor proteins (Figure 29). Although, the sensor proteins established that MG132 treatment results in severe disruption of cellular proteostasis, they cannot report the changes occurring in different subcellular compartments. To measure the effect of proteasome inhibition on the proteostasis of cytosol and nucleus, we treated the cells stably expressing NLS- or NES-Fluc-EGFP variants with either DMSO, as a control, or $5 \mu \mathrm{M}$ MG132 for $8 \mathrm{~h}$ (Figure 40).

In the DMSO controls, diffuse distribution of EGFP fluorescence was observed in the nucleus or in the cytosol of cells expressing NLS- or NES-Fluc-EGFP variants, respectively (Figure 40). Fluc-EGFP variants without signal sequence were uniformly distributed both in the nucleus and in the cytosol. Upon proteasome inhibition, almost all cells expressing NLS-Fluc sensors showed massive aggregation in the nucleus (Figure 40). The aggregation of wild-type Fluc in the nucleus suggests that the nuclear environment is highly sensitive to proteostasis imbalance. Under the same treatment conditions, only 40-50\% of the cells expressing NES-FlucEGFP formed aggregates, which were localized mainly in the cytosol. However, contrary to our expectations, $70-80 \%$ of the cells expressing NES-FlucDM-EGFP showed aggregates both in the nucleus and the cytosol, whereas only $30 \%$ of the cells formed aggregates exclusively in the cytosol. This observation that NES-Fluc-EGFP formed aggregates mainly in the cytoplasm while NES-FlucDM-EGFP formed aggregates both in the nucleus and the cytoplasm suggests that there is a differential effect of proteasome inhibition on the stability and subcellular distribution of aggregates of NES-Fluc-EGFP variants (Figure 40). 


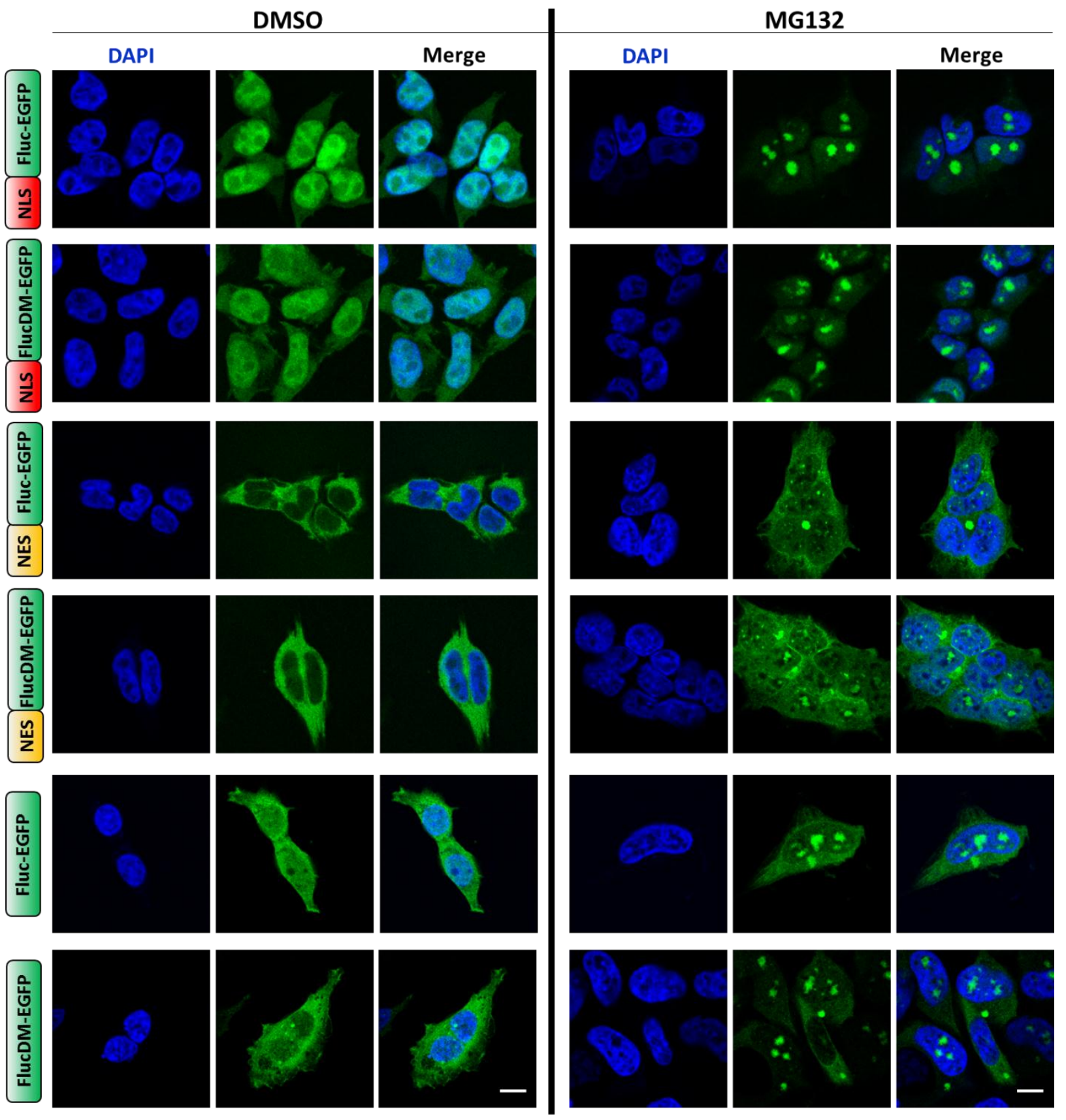

Figure 40: NES-FlucDM-EGFP forms aggregates in the nucleus upon proteasome inhibition.

Representative confocal micrographs of HEK 293T cells stably expressing indicated Fluc-EGFP constructs. The cells were treated with either $0.1 \%$ DMSO or $5 \mu \mathrm{M}$ MG132 for $8 \mathrm{~h}$. EGFP fluorescence is shown in green. Nuclei were stained with DAPI (blue). Scale bar corresponds to $10 \mu \mathrm{m}$.

When we followed the effect of proteasome inhibition on the subcellular distribution of Fluc-EGFP variants without signal sequence we observed that, while $40 \%$ of the cells expressing 
Fluc-EGFP showed aggregates in both cytosol and nucleus, $80 \%$ of the cells expressing FlucDM-EGFP showed aggregates predominantly in the nucleus (Figure 40). The propensity of structurally destabilized FlucDM-EGFP with an NES or without a signal sequence to form aggregates primarily in nucleus upon proteasome inhibition suggests a so far undiscovered role of the nucleus in protein quality control.

To understand whether the aggregates formed in the nucleus or whether they were imported from the cytosol, we performed live-cell imaging to investigate the aggregation kinetics of NES-Fluc-EGFP variants upon proteasome inhibition (Figure 41). Cells expressing NES-FlucEGFP began to show small aggregate-like foci in the cytosol after $2 \mathrm{~h}$ of MG132 addition. These foci increased in size but remained cytosolic until $8 \mathrm{~h}$. In contrast, cells expressing NESFlucDM-EGFP began to display aggregates in the cytosol within $1 \mathrm{~h}$ of MG132 addition and within $4 \mathrm{~h}$ in the nucleus. While the aggregates in the cytosol did not grow in size, the nuclear aggregates increased both in number and size in the nucleus until $8 \mathrm{~h}$. This suggests that NESFlucDM-EGFP aggregates are not imported from the cytosol but formed in the nucleus.

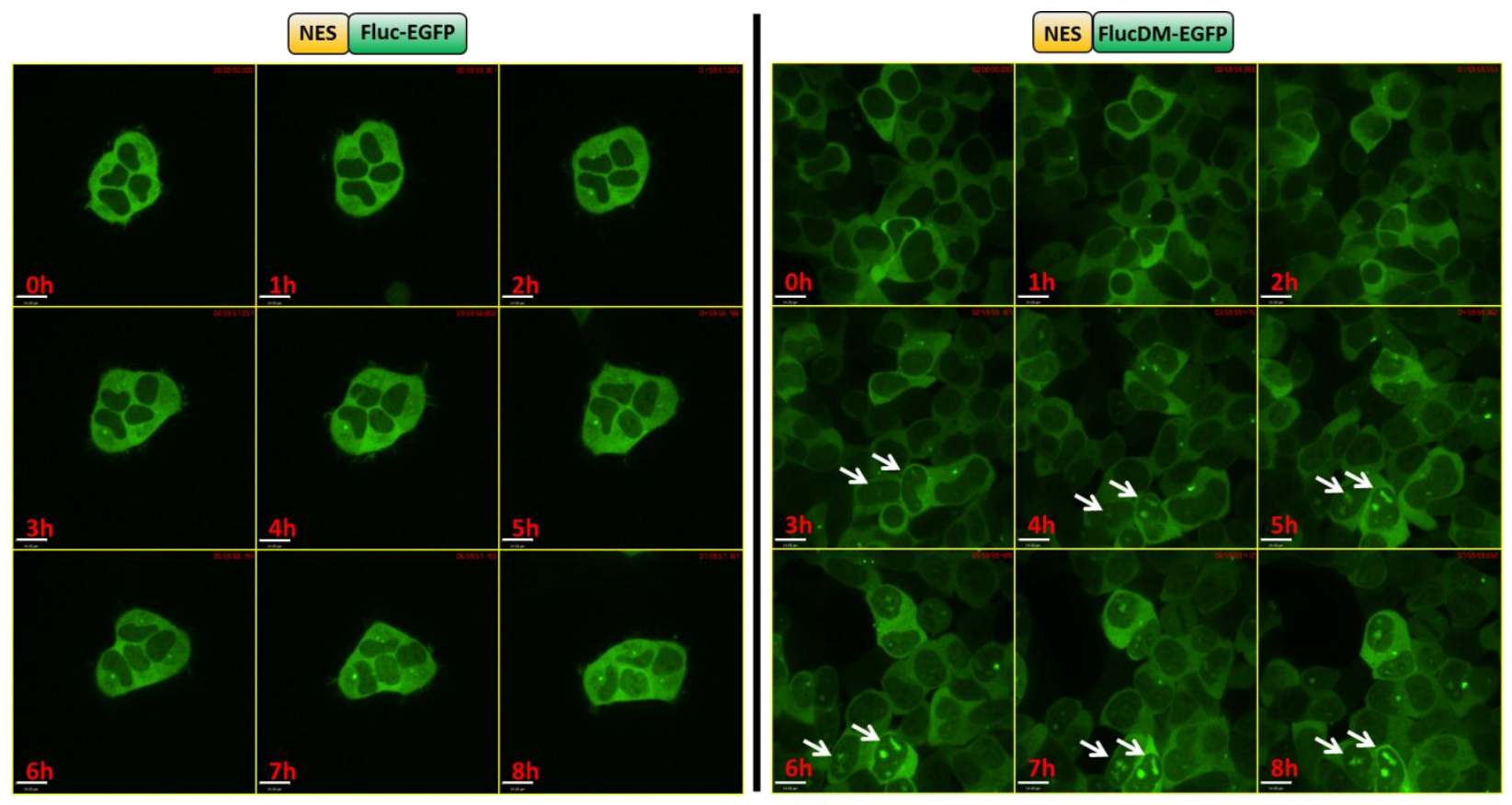

Figure 41: Time course of aggregate formation of NES-Fluc-EGFP variants upon proteasome inhibition.

Representative confocal micrographs from live cell imaging of HEK 293T cells stably expressing NESFluc-EGFP or NES-FlucDM-EGFP at the times indicated. Time point $0 \mathrm{~h}$ indicates the time of addition of $5 \mu \mathrm{M}$ MG132 to the medium. EGFP fluorescence is shown in green. White arrows indicate aggregates in the nucleus. Scale bars correspond to $14 \mu \mathrm{m}$. 


\section{V.4.5.3. Assessment of the Recovery Capacity of Nucleus and Cytosol after MG132 Removal}

To understand the recovery capacity of nucleus and cytosol from stress caused by inhibition of proteasome mediated degradation, we incubated cells expressing NLS- or NESFluc-EGFP sensor proteins in medium without MG132 for an additional $16 \mathrm{~h}$ after the MG132 treatment (recovery).



Figure 42: Differences in the recovery profile of nucleus and cytosol upon MG132 removal. Representative confocal micrographs of HEK 293T cells stably expressing indicated Fluc-EGFP constructs. The cells were fixed either immediately, after treatment with $5 \mu \mathrm{M}$ MG132 for $8 \mathrm{~h}$ or after a $16 \mathrm{~h}$ recovery period in a medium without MG132. EGFP fluorescence is shown in green. Nuclei were stained with DAPI (blue). White arrows indicate the presence of aggregates in the cytosol. Scale bars correspond to $10 \mu \mathrm{m}$.

As shown before, upon proteasome inhibition the cells expressing NLS-Fluc-EGFP variants showed dense aggregates in the nucleus, whereas cells expressing NES-Fluc-EGFP 
displayed aggregates in cytosol (Figure 42). After MG132 treatment, the majority of NESFlucDM-EGFP formed inclusions in the nucleus, and only a small subset of cells showed inclusions in the cytosol. When cells were allowed to recover from MG132 stress for $16 \mathrm{~h}$, the aggregates of NLS-Fluc-EGFP or NLS-FlucDM-EGFP in the nucleus resolved completely, but NES-Fluc-EGFP expressing cells still showed small aggregates in the cytosol. In the NESFlucDM-EGFP expressing cells, the aggregates in the nucleus resolved completely, however the aggregates in the cytosol were still present. These observations indicate that although the proteostasis capacity of the nucleus is not sufficient to prevent aggregation of proteins upon proteasome inhibition, it is highly efficient in removal of the aggregates of Fluc-EGFP sensors.

\section{V.4.5.4. Role of the Nucleolus in Stress Response}

In our fluorescence microscopy experiments, we observed that the aggregates of the NLS-Fluc-EGFP variants and NES-FlucDM-EGFP in the nucleus were excluded from DNA (DAPI negative) at specific sites in the nucleoplasm (Figure 40). To distinguish whether this absence of DNA was simply a steric effect or whether it was due to localization of aggregates to specific sites in the nucleus, we performed co-localization experiments with a marker for nucleoli.

The nucleolus is a non-membrane bound compartment in the nucleus responsible for rRNA transcription, processing and assembly of ribosomal subunits. Besides its role in ribosome biogenesis, the nucleolus has been proposed to play an active role in stress response pathways. Key stress-related proteins like Hsc70/Hsp70 immediately translocate to the nucleolus during heat stress or acidosis (Audas et al., 2012; Banski et al., 2010; Welch and Feramisco, 1984). Moreover, many cellular proteins such as p53 (Karni-Schmidt et al., 2008; Klibanov et al., 2001), promyelocytic leukemia gene product (PML) (Mattsson et al., 2001), cyclins, cyclin dependent kinases (CDKs) and several transcription factors such as Sp1 and Sp3 (Latonen et al., 2011) have been shown to accumulate in the nucleolus upon proteasome inhibition.

To test whether Fluc aggregates also localized to nucleoli after proteasome inhibition, we transiently transfected cells expressing the NLS- or NES-Fluc-EGFP variants with a mCherrytagged NPM1 construct. The NPM1 gene in humans encodes a nucleolar protein called nucleophosmin (NPM1) or nucleolar phosphoprotein B23 which participates in ribosome biogenesis and transport and is used as a marker for nucleoli (Boisvert et al., 2007; Boulon et al., 
2010). As observed before, treatment of cells with $5 \mu \mathrm{M} \mathrm{MG132}$ for $8 \mathrm{~h}$ led to the aggregation of NES-FlucDM-EGFP and all NLS-Fluc-EGFP variants in the nucleus, while NES-Fluc-EGFP formed aggregates in the cytosol (Figure 43). The nuclear aggregates co-localized with NPM1mCherry, which suggests that nuclear Fluc aggregates accumulate in the nucleolus in response to proteasome inhibition.

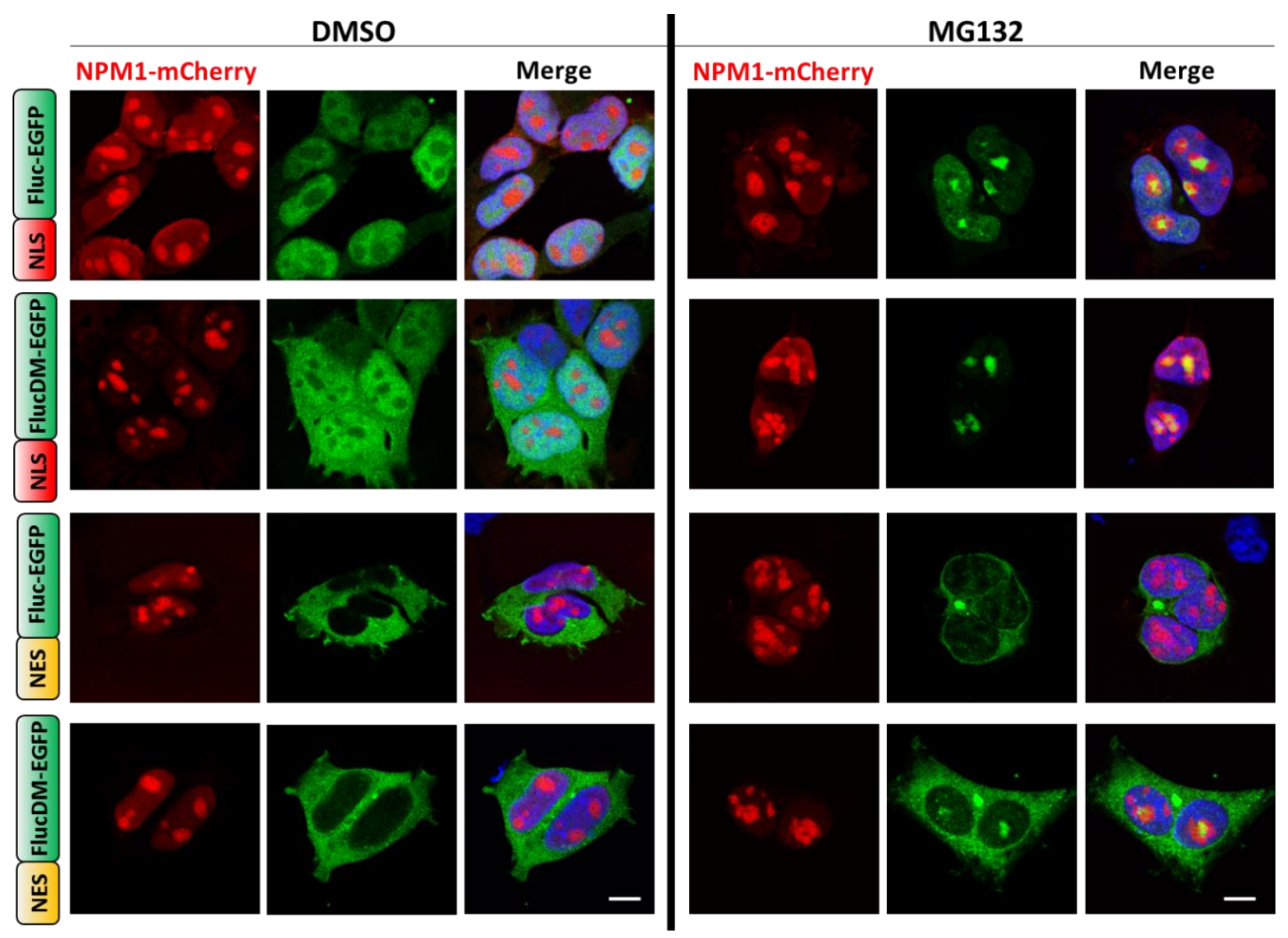

Figure 43: Accumulation of NLS-Fluc-EGFP variants and NES-FlucDM-EGFP in the nucleoli in response to proteasome inhibition.

Representative confocal micrographs of HEK 293T cells stably expressing the indicated Fluc-EGFP constructs and transiently transfected with NPMI-mCherry vector. Twenty four hours after transfection, the cells were treated with $0.1 \%$ DMSO or $5 \mu \mathrm{M}$ MG132 for $8 \mathrm{~h}$. EGFP and mCherry fluorescence are shown in green and red respectively. Nuclei were stained with DAPI (blue). Scale bars correspond to 10 $\mu \mathrm{m}$.

To test whether other forms of stress can also induce the aggregation of NLS- or NESFluc-EGFP variants in the nucleoli, we monitored the effect of heat stress $\left(43^{\circ} \mathrm{C}\right.$ for $\left.2 \mathrm{~h}\right)$ on the subcellular distribution of these proteins (Figure 44). Compared to cells maintained at $37^{\circ} \mathrm{C}$ 
where the majority of cells showed no aggregates, $80-90 \%$ of cells expressing NLS-Fluc-EGFP variants showed aggregates in nucleoli upon heat stress. In contrast to proteasome inhibition which resulted in aggregation of NES-FlucDM-EGFP predominantly in the nucleoli, heat stress induced aggregation of Fluc variants with an NES was primarily observed in the cytosol. Around $20-30 \%$ of cells expressing NES-Fluc-EGFP and 60-70\% of cells with NES-FlucDM-EGFP showed aggregates in the cytosol, and no aggregates were observed in the nucleus. This suggests that the aggregation process can be different in both compartments, and may depend on the duration and severity of cellular stresses.



Figure 44: Heat stress leads to the aggregation of NES-FlucDM-EGFP in the cytosol. Representative confocal micrographs of HEK 293T cells stably expressing indicated Fluc-EGFP constructs and transiently transfected with NPM1-mCherry. Twenty four hours after transfection, the cells were either maintained at $37^{\circ} \mathrm{C}$ or given heat stress at $43^{\circ} \mathrm{C}$ for $2 \mathrm{~h}$. EGFP and mCherry fluorescence are shown in green and red respectively. Nuclei were stained with DAPI (blue). Scale bars correspond to 10 $\mu \mathrm{m}$. 


\section{V.4.5.5. The Aggregates of Fluc-EGFP Variants Co-localize with $20 \mathrm{~S}$} Proteasome and Ubiquitin upon Proteasome Inhibition

Many studies have shown that proteotoxic stress by MG132 leads to the translocation of proteasomes to nucleoli (Latonen et al., 2011; Mattsson et al., 2001). Although direct experimental evidence supporting a role of the nucleolus in regulating proteasome mediated degradation is limited, some reports suggest that the nucleoli, directly or indirectly, influence the turn-over of some proteins, such as p53, by regulating their interaction with their binding partners (Boyd et al., 2011; Klibanov et al., 2001).

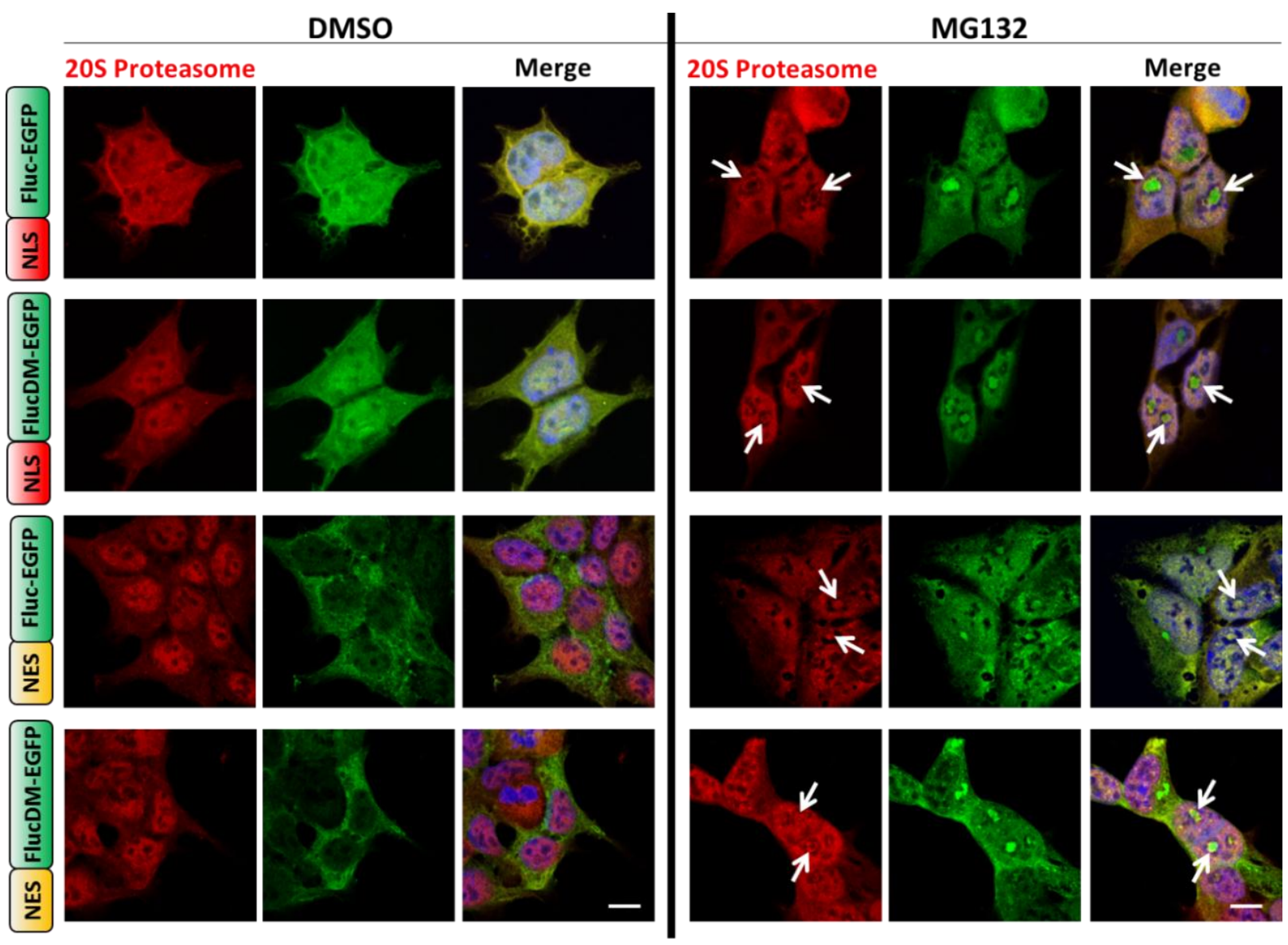

Figure 45: Co-localization of Fluc-EGFP aggregates with $20 \mathrm{~S}$ proteasome core particle.

Representative immunofluorescence confocal micrographs of HEK 293T cells stably expressing the indicated Fluc-EGFP constructs. The cells were treated with either $0.1 \%$ DMSO or $5 \mu \mathrm{M}$ MG132 for $8 \mathrm{~h}$ before fixing them with $4 \%$ paraformaldehyde. 20S proteasome core particles were detected by immunocytochemistry using a $20 \mathrm{~S}$ proteasome primary antibody followed by a $\mathrm{Cy} 3$ conjugated secondary antibody (red). Fluc-EGFP variants were detected by EGFP fluorescence and nuclei were stained with DAPI (blue). White arrows indicate the presence of $20 \mathrm{~S}$ proteasome core particle surrounding Fluc-EGFP aggregates in the nucleus. Scale bars correspond to $10 \mu \mathrm{m}$. 
To test whether aggregates of NLS- or NES-Fluc-EGFP variants co-localize with the proteasome, we employed confocal microscopy using an antibody against the 20S core particle of the proteasome (Figure 45). In DMSO control samples, the 20S proteasome core particles were uniformly distributed in cells, with slightly higher levels in the nucleus. However, upon addition of $5 \mu \mathrm{M}$ MG132 for $8 \mathrm{~h}$, the 20S core particles distributed to the nucleoli and were seen in a ring-like structure surrounding the aggregates of NLS- or NES-Fluc-EGFP proteins.

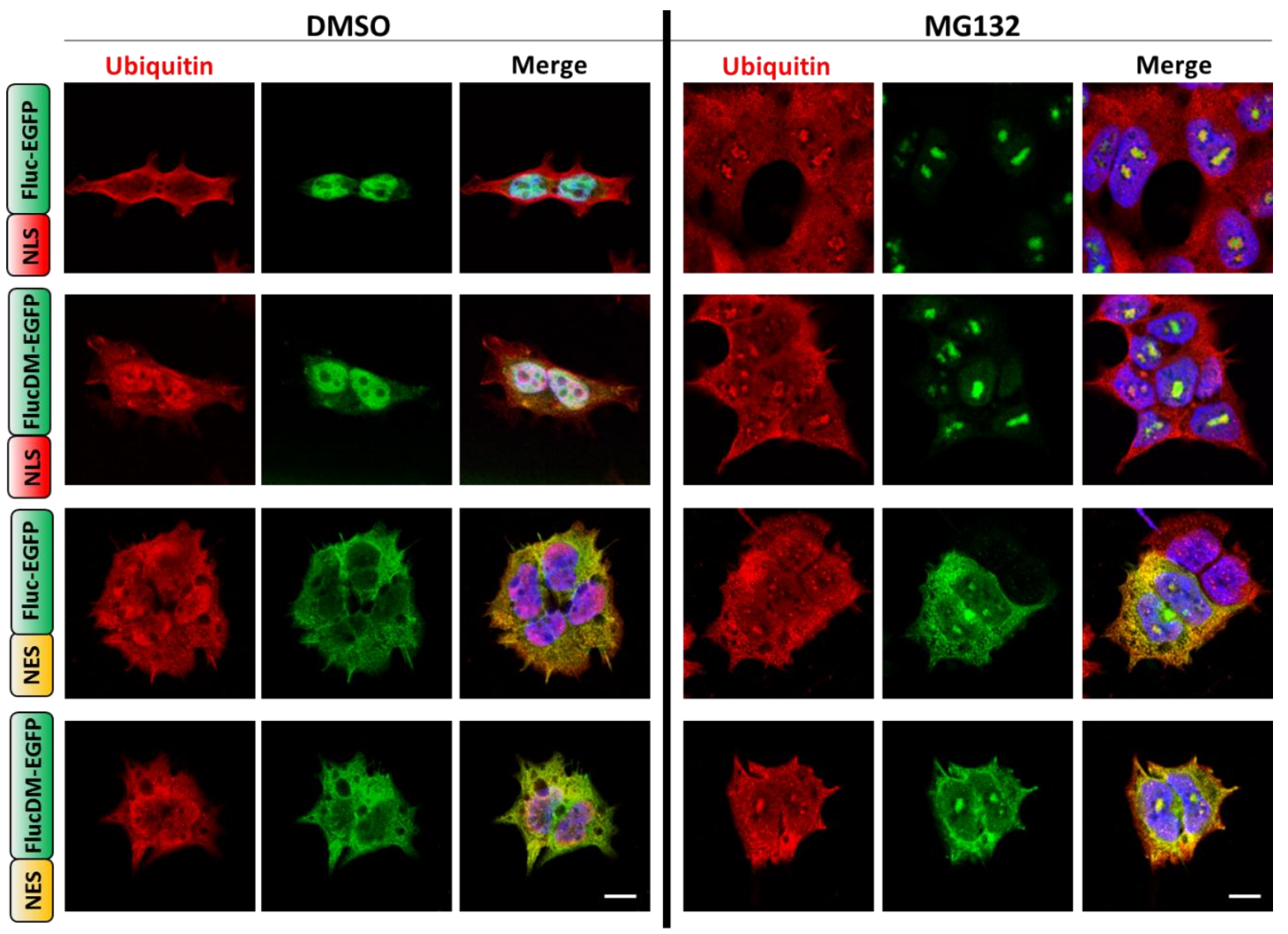

Figure 46: Fluc-EGFP aggregates in both nucleus and cytosol co-localize with ubiquitin.

Representative immunofluorescence confocal micrographs of HEK 293T cells stably expressing indicated Fluc-EGFP constructs. The cells were treated with either $0.1 \%$ DMSO or $5 \mu \mathrm{M}$ MG132 for $8 \mathrm{~h}$ before fixing them with $4 \%$ paraformaldehyde. Ubiquitin was detected by immunocytochemistry using ubiquitin primary antibody (clone FK2) followed by Cy3 conjugated secondary antibody (red). Fluc-EGFP variants were detected by EGFP fluorescence and nuclei were stained with DAPI (blue). Scale bar corresponds to $10 \mu \mathrm{m}$.

Having observed that Fluc-EGFP aggregates co-localize with the $20 \mathrm{~S}$ proteasome, we next tested if these aggregates also co-localize with ubiquitin. We selected an ubiquitin antibody 
that recognizes ubiquitin-conjugated proteins but not free ubiquitin. Like the $20 \mathrm{~S}$ proteasome, ubiquitin also co-localized with Fluc-aggregates upon MG132 stress, indicating that they may be ubiquitinated (Figure 46). The co-localization of both 20S proteasomes and ubiquitin with Flucbased sensors in the nucleoli of MG132 treated cells suggests that Fluc proteins may be transported to the nucleoli under normal conditions and that the nucleolus may have a function in the regulation of proteasome mediated protein degradation.

\section{V.4.5.6. Role of the Nucleus in the Degradation of NES-FlucDM-EGFP}

The aggregation of the cytosolic protein NES-FlucDM-EGFP in the nucleus/nucleolus upon proteasome inhibition despite its high concentration in the cytosol was a surprising result. Additionally, the co-localization of NES-FlucDM-EGFP aggregates with 20S proteasome core particles and ubiquitin suggested a role of the nucleus in the degradation of cytosolic misfolded proteins. The hypothesis is in line with a study in yeast which showed that degradation of cytosolic misfolded carboxypeptidase Y (CPY*) occurs in the nucleus (Prasad et al., 2010). To understand if the cellular localization of the protein has an influence on its degradation, we measured the degradation kinetics of NES-FlucDM-EGFP in the nucleus, by performing a cycloheximide (CHX) chase experiment in the presence of an inhibitor of nuclear export.

Proteins containing an NES sequence are maintained cytosolic by constitutive action of the CRM1/exportin mediated nuclear export cycle (Fornerod et al., 1997; Fukuda et al., 1997). The CRM1 protein, together with RanGTP, binds to leucine-rich NES containing proteins in the nucleus and transports them out of the nucleus through the nuclear pore. After exit from the nucleus, the hydrolysis of RanGTP to RanGDP by RanGTPase causes the dissociation of CRM1cargo complex and thereby releases the cargo into the cytoplasm. This export cycle can be inhibited by the small molecule leptomycin B (LMB) which alkylates Cys529 of CRM1 and blocks its function (Kudo et al., 1999; Kudo et al., 1998).

NES-FlucDM-EGFP cells treated with DMSO show a uniform distribution of EGFP fluorescence predominantly in the cytosol, whereas cells treated with LMB showed EGFP fluorescence both in the cytosol and the nucleus (Figure 47a). This observation indicates that LMB was effective in inhibiting the nuclear export cycle (Figure 47a). Next, we measured the degradation kinetics of NES-FlucDM-EGFP for up to $9 \mathrm{~h}$ by blocking the synthesis of new protein through addition of $0.5 \mathrm{mM} \mathrm{CHX}$. Under these conditions, the half-life $\left(\mathrm{t}_{1 / 2}\right)$ of NES- 
FlucDM-EGFP was $\sim 6 \mathrm{~h}$. These conditions should mainly measure the degradation of NESFlucDM-EGFP in the cytosol (Figure 47b, c). However, when LMB and CHX were added together, the $\mathrm{t}_{1 / 2}$ of NES-FlucDM-EGFP was reduced to $\sim 3 \mathrm{~h}$ which indicates faster degradation of the protein when it is retained in the nucleus (Figure 47b, c). There was no significant change in the amount of NES-FlucDM-EGFP in cells treated with LMB alone, indicating that LMB alone does not influence protein degradation. This result suggests that degradation of NESFlucDM-EGFP is enhanced when it is selectively retained in the nucleus compared to its normal degradation in the cytosol.

a

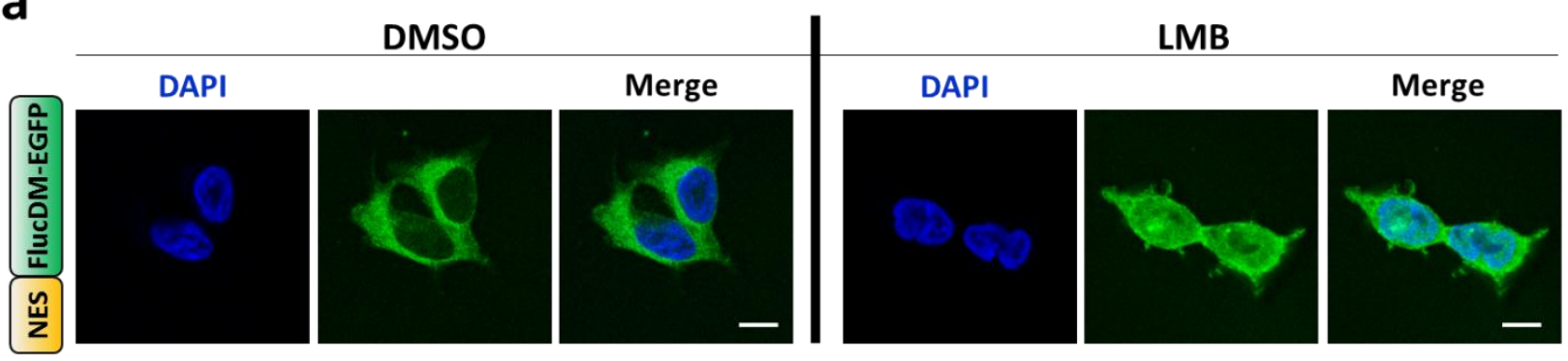

b



C



Figure 47: Leptomycin B (LMB) treatment leads to increased levels of NES-FlucDM-EGFP in the nucleus and accelerates it degradation.

(a) Representative confocal micrographs showing subcellular distribution of NES-FlucDM-EGFP in HEK 293T cells treated with either $0.1 \%$ DMSO or $10 \mathrm{ng} / \mathrm{ml}$ LMB for $8 \mathrm{~h}$. NES-FlucDM-EGFP was visualized by GFP fluorescence (green) and nuclei were stained with DAPI (blue). Scale bars correspond to $10 \mu \mathrm{m}$. (b) NES-FlucDM-EGFP levels in HEK 293T cells treated with $0.5 \mathrm{mM}$ cycloheximide (CHX), $10 \mathrm{ng} / \mathrm{ml}$ leptomycin B (LMB) or combination of both $(\mathrm{CHX}+\mathrm{LMB})$ for the times indicated. Amount of NESFlucDM-EGFP at time point $0 \mathrm{~h}$ is set to $100 \%$. Error bars indicate s.d., $\mathrm{n}=3$. (c) Representative immunoblots showing the levels of NES-FlucDM-EGFP (probed with anti-GFP antibody) in HEK 293T cells treated as above. GAPDH was used as a loading control. 


\section{V.4.5.7. Effect of DnaJB1 Down-regulation on the Degradation of NES- FlucDM-EGFP}

The results presented in Figure 47 as well as findings using CPY* in yeast (Prasad et al., 2010) suggest a model, where misfolded cytosolic proteins move to the nucleus for their degradation. This model would predict that some cellular factor can recognize these misfolded proteins in the cytosol and direct them to the nucleus for degradation.

Due to their ability to recognize and bind to misfolded proteins, chaperones are a likely candidate for such a function. It is well established that chaperones along with co-chaperones recognize misfolded proteins and prevent their accumulation either by refolding them or targeting them for degradation. The triage decision to refold or degrade a substrate relies on the interaction of a co-chaperone with its chaperone. Among chaperones, the Hsp70-Hsp40 system is extensively studied and well characterized both in vitro and in vivo.

Hsp40 (DnaJ), besides stimulating the ATPase activity of Hsp70, has been shown to play a role in stress response pathways. It has been shown in HeLa cells that DnaJB1 and Hsp70 translocate to nucleoli upon heat stress (Hattori et al., 1993; Terada and Mori, 2000). Moreover, it has been shown that DnaJB1 co-localizes with nuclear inclusions of polyQ proteins (Chai et al., 1999; Seidel et al., 2011) and that it can facilitate the degradation of some substrates (Bailey et al., 2002). Therefore, we investigated the effect of Hsp40/DNAJB1 on the folding and degradation status of NES-FlucDM-EGFP.

We studied the effect of DnaJB1 on the degradation kinetics of NES-FlucDM-EGFP by transfecting cells with either DnaJB1 siRNA or control siRNA for $72 \mathrm{~h}$, followed by a CHX chase experiment in the presence or absence of LMB. The down-regulation of DnaJB1 by siRNA treatment depleted endogeneous DnaJB1 by 50-60\% (Figure 48b). The $\mathrm{t}_{1 / 2}$ of NES-FlucDMEGFP was $\sim 2 \mathrm{~h}$ in control siRNA transfected cells treated with both CHX and LMB, compared to $\sim 4 \mathrm{~h}$ in cells treated with $\mathrm{CHX}$ alone (Figure 48), supporting the previous results which showed that degradation is enhanced when NES-FlucDM-EGFP is retained within the nucleus. In cells transfected with DnaJB1 siRNA, a significant stabilization of NES-FlucDM-EGFP was observed. The $\mathrm{t}_{1 / 2}$ was increased to $\sim 4 \mathrm{~h}$ in cells treated with CHX and LMB and up to $9 \mathrm{~h}$ in cells treated with CHX alone; suggesting that DnaJB1 is involved in the degradation of NES- 
FlucDM-EGFP and that its down-regulation stabilizes the protein both in the nucleus and the cytosol.
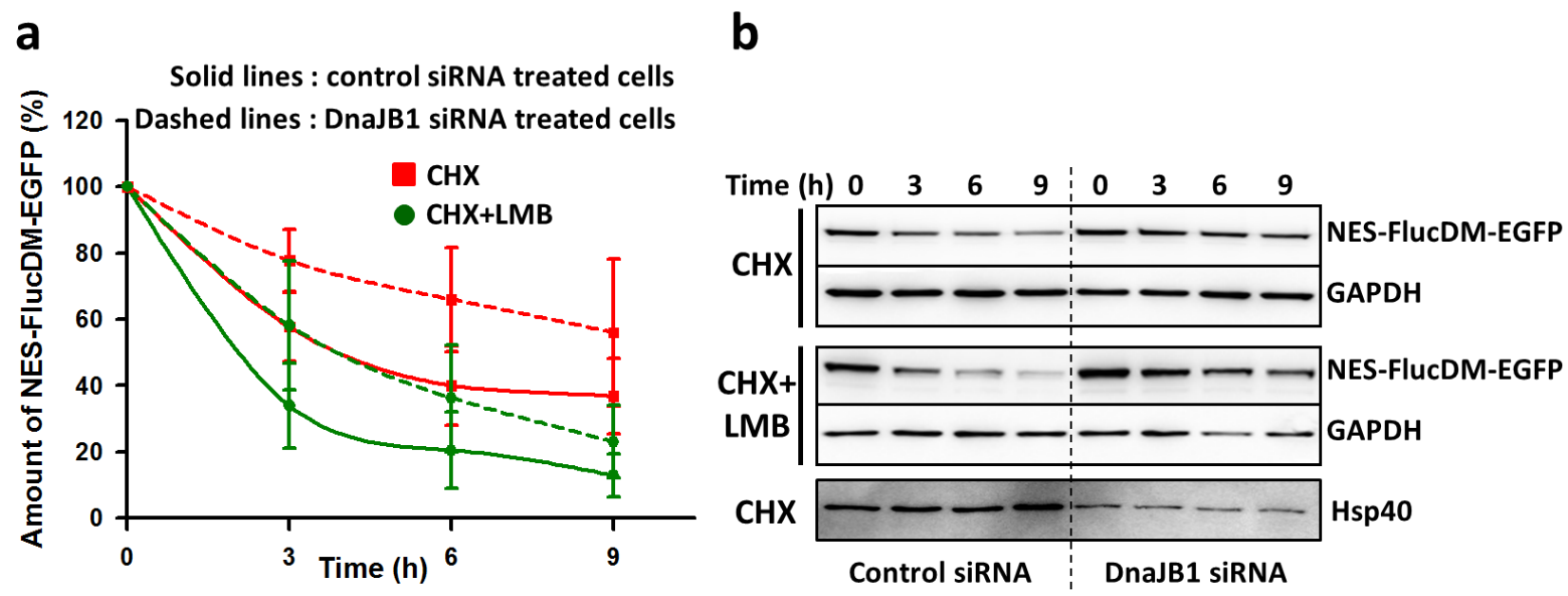

Figure 48: Degradation of NES-FlucDM-EGFP is dependent on DnaJB1 (Hsp40).

(a) Amount of NES-FlucDM-EGFP in cells transfected with either control or DnaJB1 siRNA and treated with $0.5 \mathrm{mM}$ cycloheximide (CHX) alone or together with $10 \mathrm{ng} / \mathrm{ml}$ leptomycin $\mathrm{B}(\mathrm{CHX}+\mathrm{LMB})$ at the times indicated. Amount of NES-FlucDM-EGFP at time point $0 \mathrm{~h}$ set to $100 \%$. Error bars indicate s.d., $\mathrm{n}=3$. (b) The representative immunoblots showing the levels of NES-FlucDM-EGFP (probed with antiGFP antibody) and Hsp40 (probed with anti-Hsp40 antibody) in cells transfected with either control or DnaJB1 siRNA and treated as above. GAPDH was used as a loading control.

\section{V.4.5.8. Effect of DnaJB1 Down-regulation on the Subcellular Localization of NES-FlucDM-EGFP Aggregates upon Proteasome Inhibition}

To check the effect of DnaJB1 on the subcellular distribution of NES-FlucDM-EGFP aggregates upon proteasome inhibition, we transfected cells with either control siRNA or DnaJB1 siRNA for $72 \mathrm{~h}$, followed by treatment with $5 \mu \mathrm{M}$ MG132 or DMSO for $8 \mathrm{~h}$ (Figure 49).

DnaJB1 down-regulation resulted in aggregation of NES-FlucDM-EGFP in the cytosol of $\sim 25 \%$ cells treated with DMSO, supporting previous findings that Fluc is dependent on the Hsp70-Hsp40 system for its folding (Frydman et al., 1994; Schroder et al., 1993). Upon proteasome inhibition, in cells transfected with control siRNA, the aggregates of NES-FlucDMEGFP were observed predominantly in the nucleus, in contrast to cells with DnaJB1 downregulation where aggregates were predominantly cytosolic. These observations suggest that DnaJB1 may recognize and transport misfolded forms of NES-FlucDM-EGFP into the nucleus 
for degradation and that its down-regulation therefore causes the accumulation of Fluc variant in the cytosol.

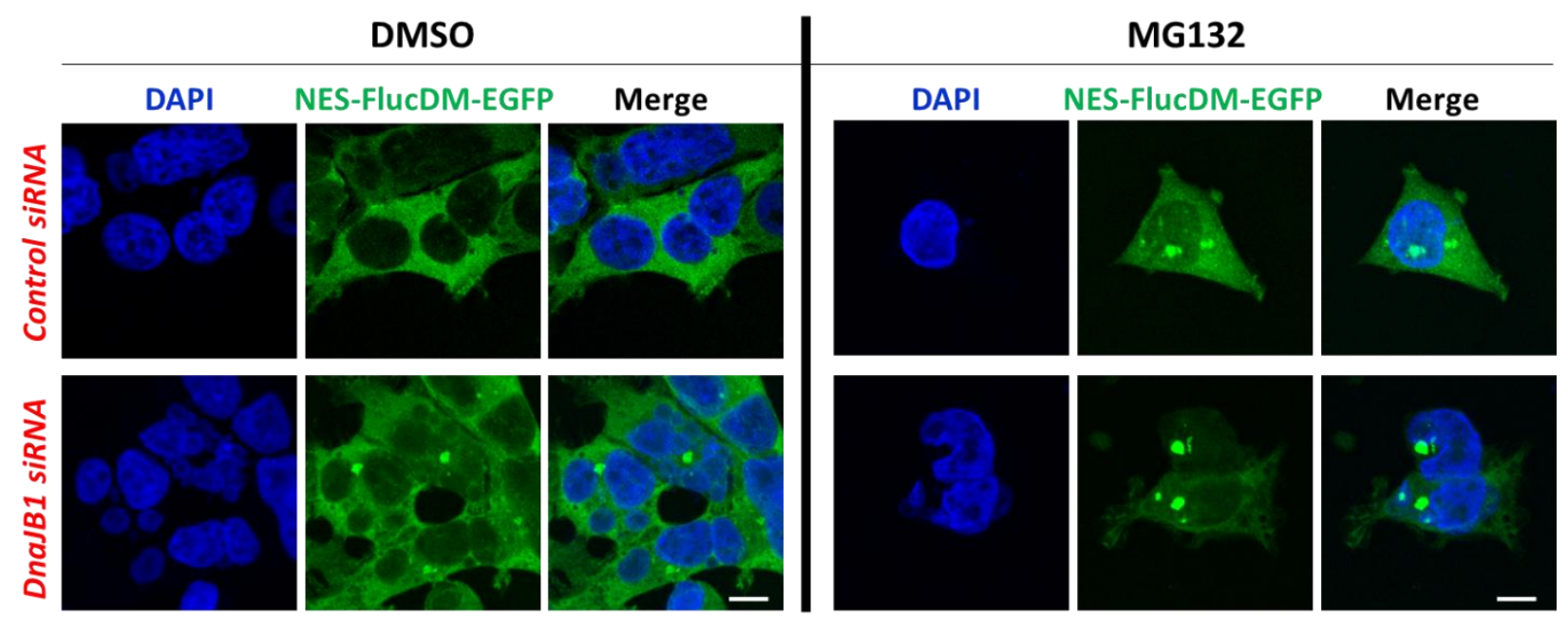

Figure 49: DnaJB1 knockdown leads to the accumulation of NES-FlucDM-EGFP in the cytosol upon proteasome inhibition.

Representative confocal micrographs showing the subcellular localization of NES-FlucDM-EGFP in HEK 293T cells transfected with either control or DnaJB1 siRNA and treated with $0.1 \%$ DMSO or $5 \mu \mathrm{M}$ MG132 for $8 \mathrm{~h}$. NES-FlucDM-EGFP was visualized by GFP fluorescence (green) and nuclei were stained with DAPI (blue). Scale bars correspond to $10 \mu \mathrm{m}$.

\section{V.4.5.9. Effect of a Pharmacological Chaperone on the Stability of NLS- and NES-Fluc-EGFP Variants}

It has been shown previously that small molecule compounds that can help proteins in acquiring their native state may have great therapeutic potential. These compounds, called pharmacological chaperones (PC), are highly specific and can rescue proteins from loss-offunction phenotypes ( $\mathrm{Mu}$ et al., 2008). Therefore, small molecules that resemble natural substrates may act as PC for enzymes. The small chemical compound 2-phenyl benzothiazole (PBT) is structurally similar to the natural Fluc substrate D-luciferin and can act as a substrate analog (Figure 50a). PBT has been shown to competitively inhibit Fluc by binding specifically to the luciferin binding site in vitro with a $\mathrm{K}_{\mathrm{I}}=0.015 \mu \mathrm{M}$. It has also been shown that PBT significantly enhances the stability of purified luciferase in vitro (Thompson et al., 1991).

To test whether PBT can act as a PC for the Fluc-based sensors, especially for the destabilized variants NLS- or NES-FlucDM-EGFP, we determined the levels of Fluc-EGFP- 
variants in presence or absence of PBT (Figure 50b). In all NLS- or NES-Fluc-EGFP variants, there was a 4-5 fold increase in the levels of Fluc-proteins after a $9 \mathrm{~h}$ incubation of cells with PBT, which suggests that PBT significantly stabilizes the conformation of wild-type and mutant Fluc-proteins.
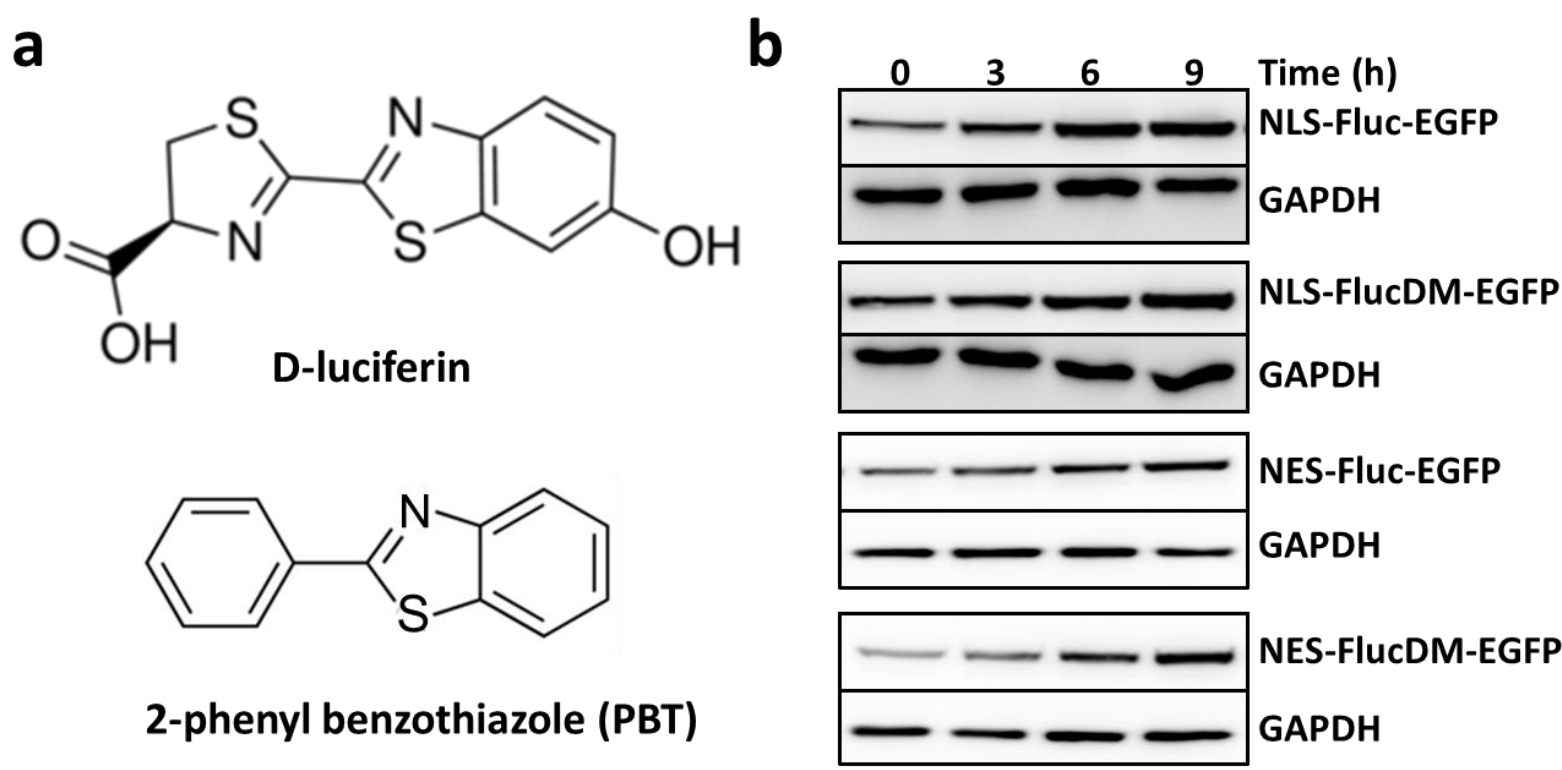

NES-Fluc-EGFP GAPDH

\section{2-phenyl benzothiazole (PBT)}

\section{NES-FlucDM-EGFP}

GAPDH

\section{Figure 50: 2-phenyl benzothiazole stabilizes Fluc-EGFP variants.}

(a) Chemical structures of D-luciferin and 2-phenyl benzothiazole (PBT), a competitive inhibitor of Fluc. (b) Representative immunoblots showing the levels of NLS- or NES-Fluc-EGFP variants in HEK 293T cells treated with $0.1 \%$ DMSO or $40 \mu \mathrm{g} / \mathrm{ml}$ PBT for the indicated times. Fluc-EGFP variants were detected by anti-GFP antibody and GAPDH was used as a loading control.

To test whether PBT can prevent Fluc-based sensors from MG132 induced aggregation, we treated cells expressing NLS- or NES-Fluc-EGFP variants with $5 \mu \mathrm{M}$ MG132 and PBT for 8 h (Figure 51). As shown before, cells treated with MG132 alone showed aggregates of all FlucEGFP variants. However, proteasome inhibition in the presence of PBT resulted in only small and few aggregates in the nucleus in cells expressing NLS-Fluc-EGFP variants, and no aggregates could be observed in cells expressing the NES-Fluc-EGFP variants. These observations suggest that PBT significantly stabilizes Fluc variants and reinforces the concept of pharmacological chaperones in preventing protein misfolding. 


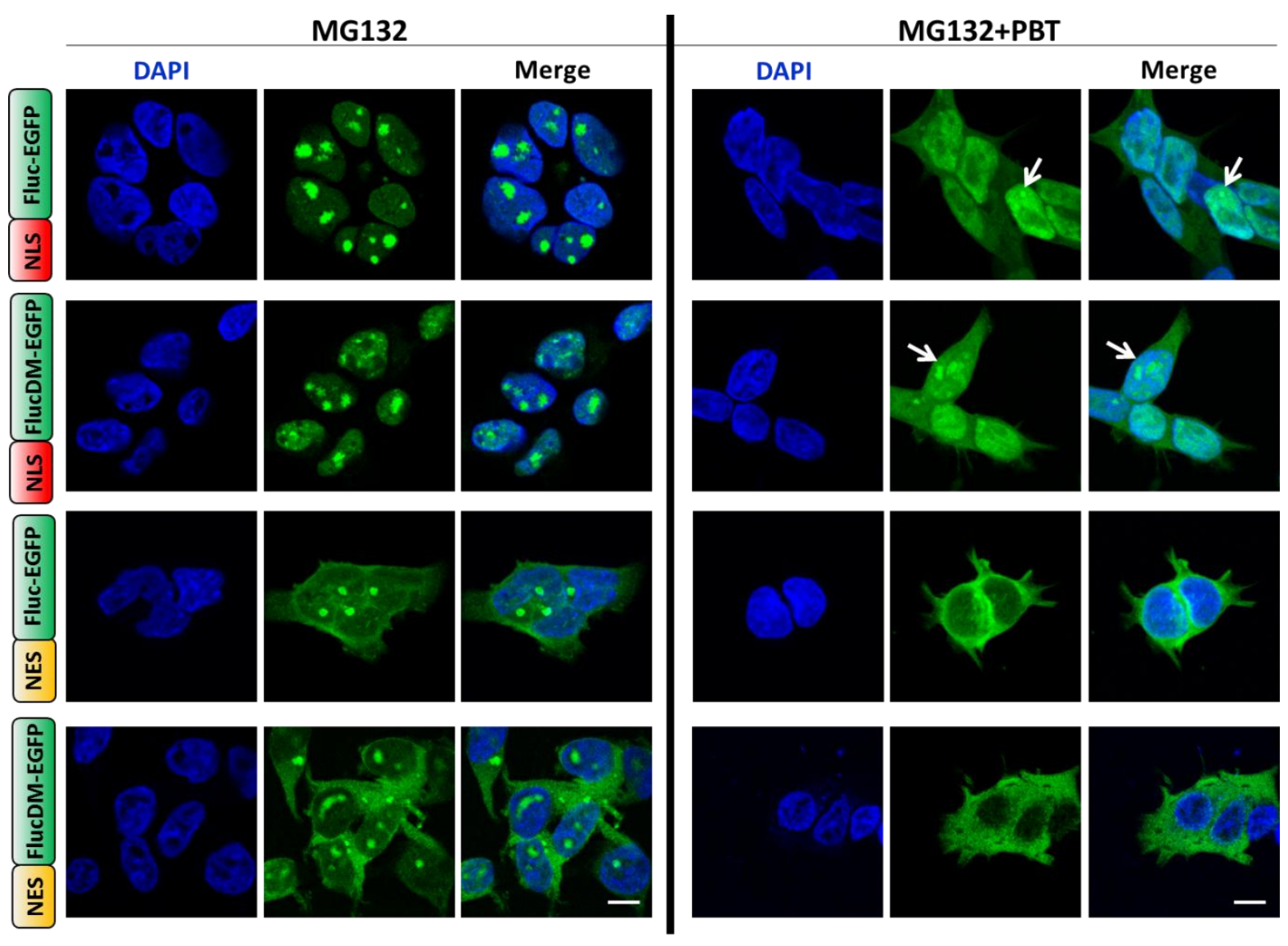

Figure 51: 2-phenyl benzothiazole prevents aggregation of Fluc-EGFP variants caused by MG132 treatment.

Representative confocal micrographs of HEK $293 \mathrm{~T}$ cells stably expressing the indicated Fluc-EGFP constructs and treated with $5 \mu \mathrm{M}$ MG132 alone or together with $40 \mu \mathrm{g} / \mathrm{ml}$ PBT for $8 \mathrm{~h}$. EGFP fluorescence is shown in green and nuclei were stained with DAPI (blue). White arrows indicate the presence of aggregates in the nucleus. Scale bars correspond to $10 \mu \mathrm{m}$. 


\section{Discussion}

Protein homeostasis or proteostasis describes the ability of a cell to maintain and protect its proteome in a biologically active functional state during normal and adverse conditions. Conditions such as environmental stress, mutations or genetic polymorphism cause protein misfolding and consequently lead to loss-of-function or toxic gain-of-function phenotypes. Therefore, a fine balance between folded and misfolded protein species is essential for the survival of cells. This is achieved by several dedicated proteins including molecular chaperones and their regulators as well as components of the ubiquitin-proteasome and autophagy systems. Together these proteins constitute the proteostasis network (PN). Deficiency in cellular proteostasis capacity has been implicated in numerous human neurodegenerative diseases such as HD, PD and ALS which are caused by protein misfolding and aggregation. Many of these diseases affect preferentially the elderly, which is consistent with a gradual decline in the efficiency of protein quality control mechanisms during aging (Gidalevitz et al., 2006; Morimoto and Cuervo, 2009).

To prevent protein misfolding and to maintain integrity of the proteome, cells express proteins such as HSF1 in cytoplasm and IRE1 $\alpha$, PERK and ATF6 $\alpha$ in the ER membrane which behave as natural sensors of proteostasis. Protein misfolding in the cytoplasm causes the activation of the HSF1 mediated heat shock response which results in increased transcription of heat shock genes. In the past, HSF1 activation was often measured to assess the status of the PN (Abravaya et al., 1991). There are different ways to measure for the activation of HSF1. Gel mobility shift assays monitor binding of HSF1 to DNA, immunoblotting can be used to measure the phosphorylation status of HSF1. Alternatively, it is possible to measure the protein and mRNA levels of HSPs, or to use a suitable reporter system driven by the HSP70 promoter. However, HSF1 activity reflects the global degree of proteome stress in cells, and cannot be used to assess subtle changes at the level of individual components of the PN. Moreover, some toxic and aggregation prone proteins such as mutant huntingtin (Htt) (Hay et al., 2004; Hipp et al., 2012; Zourlidou et al., 2007) or artificial $\beta$-sheet proteins (Olzscha et al., 2011) do not induce the heat shock response and might even suppress it. Therefore, HSF1 activity cannot be used to infer the changes in cellular proteostasis under these conditions. Protein misfolding in the ER results in an activation of the unfolded protein response (Walter and Ron, 2011) which can be semi- 
quantitatively measured by reporter based assays (Merksamer et al., 2008). The activation of heat shock response and unfolded protein response indicate that a response has been initiated but does not report whether homeostasis is restored. The relatively low sensitivity of these proteins in reporting and measuring the global changes in the $\mathrm{PN}$ in different physiological states necessitates the development of exogenous sensors.

Ideally, a sensor is able to quantitatively measure changes in proteostasis during acute stress conditions such as heat stress or during chronic stresses, including aging. Several sensors have been used to report on PN alterations. In C. elegans, temperature sensitive mutants of the muscle proteins, $\alpha$-paramyosin and $\alpha$-myosin, which are tissue-specific and can lead to loss-offunction effects, have been used to measure the folding capacity of cells during heat stress and upon expression of mutant Htt protein (Ben-Zvi et al., 2009; Gidalevitz et al., 2006). Also, proteins such as GFP-CL1, which are limited to reporting changes in specific components of the PN have been employed to measure the degradation capacity of cells (Bence et al., 2001; Nonaka and Hasegawa, 2009). The major limitation in using these sensors is their inability to report the global changes occurring in the PN. Accordingly, they are not useful in comparing proteostasis in different cells/tissues and in different cellular organelles.

To avoid these limitations, we selected Firefly luciferase (Fluc) as a sensor protein to study proteostasis. Fluc has no known biological role in the widely used cellular and animal models, and thus the sensors derived from it can be used with minimal perturbation of the system under investigation. Additionally, its enzymatic activity can be measured by a luminescencebased assay with exquisite sensitivity over a wide dynamic range in cell extracts, intact cells and model organisms. To make Fluc more sensitive to the cellular environment, we introduced point mutations to generate proteins with varying conformational stability. We also created Fluc mutants tagged with EGFP to further enhance the application of these proteins in cell biology experiments. In this study, we used Fluc-EGFP based sensors to monitor the changes in cellular protein homeostasis when different nodes of the PN, specifically folding, refolding and degradation components were altered. We further attached a nuclear localization sequence (NLS) or nuclear export sequence (NES) at the N-terminus of the Fluc-EGFP variants to study the proteostasis capacity of the nucleus and the cytoplasm. We believe that our Fluc-EGFP based sensors can be used to measure the effect and potency of small molecules on the PN. Chemical 
screens can be designed to identify proteostasis-regulating compounds that improve the activity of these sensor proteins. Moreover, the sensors can be used in genetic screens to identify suppressors and/or enhancers of cellular proteostasis capacity in different model organisms.

In the following sections, we will discuss in detail the results obtained during this study and the future perspectives regarding the applications of Fluc-based sensors in understanding proteostasis.

\section{VI.1. Fluc Mutants Are Structurally and Functionally Destabilized}

The thermostability of a protein can be modulated by substitution of amino acids to Pro and Gly which can decrease or increase the flexibility of the polypeptide chains (Matsui and Harata, 2007; Matthews et al., 1987). Besides hydrophobic interactions and the packing density of the molecules, the number of ion pairs and hydrogen bonds in proteins equally contributes to their thermostability. This is evident from the presence of large numbers of ion pairs in thermostable proteins such as those from hyperthermophilic archaea where ionic interactions prevent protein unfolding at higher temperatures (Petsko, 2001; Vieille and Zeikus, 2001). The role of hydrogen bonds in the stability of proteins has long been known and been demonstrated using various biophysical techniques. It is known that in an unfolded protein, polar amino acids form hydrogen bonds with the surrounding water molecules whereas, in a folded protein, these amino acids form intramolecular hydrogen bonds which facilitates in the folding process (Myers and Pace, 1996; Stickle et al., 1992). Therefore both, electrostatic interactions between ion pairs and intramolecular hydrogen bonding, play an important role in the stability of proteins.

In this study, we tried to destabilize the native state of Fluc by disrupting the salt bridges and hydrogen bond interactions in its N-terminal domain. We created site-specific mutations in Fluc at conserved residues, excluding positions near the enzyme's active site and at the domaindomain interface to maintain the quantum yield of the luminescence reaction (Figure 13). The positively charged residues Lys135, Arg188 and Arg261 were substituted with either polar uncharged amino acids such as Gln (K135Q, R188Q and R261Q) or with the hydrophobic amino acid Met (K135M) to disrupt the salt bridges with their corresponding ion pairs located distant in the Fluc sequence. In two cases, R188K and R261K, the aim of the mutation was to break the hydrogen bonds (due to shorter length of side chain of Lys compared to Arg) without disturbing the salt bridge between ion pairs. Thus, we aimed to decrease the stability of wild-type Fluc by 
weakening the intramolecular polar contacts in its large N-terminal domain. We generated 6 single and 12 double mutants from wild-type Fluc.

We tested the effect of mutations on the structural and functional stability of Fluc through a series of in vitro experiments using translation in rabbit reticulocyte lysate (RRL). Here we observed that mutations had a significant effect on the thermostability of the Fluc single and double mutants. The effect of double mutations on the stability of Fluc was more severe than of single mutations. This is most likely due to the synergistic effect of the loss of two polar contacts (Figure 15 and 16). To test whether the decrease in the enzymatic activities of Fluc mutants in RRL is due to their degradation, we also measured specific enzyme activities. We define specific activity as the enzymatic activity of Fluc normalized to its protein amount (quantified from immunoblotting). Intriguingly, measurement of the specific activity of Fluc mutant proteins in $\mathrm{RRL}$ at $30^{\circ} \mathrm{C}$ showed that the proteins were able to fold properly into their functional active state, but were no longer able to maintain this form (Figure 17). This observation confirms that mutations don't affect the quantum yield of the luminescence reaction but introduce a thermodynamic instability (due to the loss of the polar contacts). This is further enhanced at higher temperatures. Moreover, the results obtained from the structural characterization of these Fluc mutants by a proteinase $\mathrm{K}$ digestion assay and guanidinium chloride denaturation (Figure 20) correlated the effect of structural flexibility with enzymatic activity.

Our results show that the mutations don't significantly affect the functional properties of the Fluc mutants under normal conditions but introduce a degree of conformational flexibility in their structures. In cells, these mutations can be buffered by the action of molecular chaperones which retain these proteins in their conformationally compromised, yet functional state. Therefore it is reasonable to assume that these structurally destabilized Fluc mutants in cells depend on cellular folding factors to maintain their native state. Any stress that may cause titration of these factors is expected to result in destabilization of the mutant proteins to varying degrees. Thus, the activity read-out of Fluc mutant proteins under these conditions will measure the degree of stress on the protein quality control machinery and therefore Fluc sensors may act as "cellular thermometers". 


\section{VI.2. Assessment of Folding Capacity of Cells Using Fluc-Based Sensors}

In cells, there is a constant generation of destabilized proteins due to translational errors and genetic mutations. Consequently, a metastable sub-proteome exists that is particularly vulnerable to proteotoxic stress (Olzscha et al., 2011). Given the fact that $\sim 18 \%$ of proteins contain at least one missense substitution (Drummond and Wilke, 2008) and nearly 20-30\% of all proteins in mammalian cells are intrinsically disordered (Dunker et al., 2008), a substantial fraction of the human proteome is always at risk of misfolding. To represent the stable and metastable proteome of cells, we selected three Fluc variants on the basis of their stabilities (Figure 18); the most stable wild-type (Fluc), the moderately stable single mutant R188Q (FlucSM) and the least stable double mutant R188Q+R261Q (FlucDM). Using these three Fluc variants, we measured the effect of different stresses on the stable and the metastable cellular proteome.

Since Fluc mutant proteins are conformationally destabilized, they are good chaperone substrates and reporters to measure subtle changes in the folding capacity of cells. We determined the stability of the three Fluc variants in HeLa cells by measuring their specific activities. The results showed that a substantial fraction of mutant proteins was misfolded (Figure 22), but not aggregated (Figure 25), under normal growth conditions. The misfolding of mutant proteins could be due to the macromolecular crowding in cells which is known to significantly increase the collision between partially folded intermediates (Ellis, 2006). Our results suggest that the proteostasis capacity of HeLa cells is sufficient to prevent Fluc variants from forming aggregates, possibly by continual removal of their non-native states by degradation. Upon downregulation of the constitutive chaperone Hsc70, the specific activity of all Fluc variants further decreased (Figure 22), which supports previous findings that Fluc is dependent on Hsc70 for its folding and can be used to monitor changes in the folding capacity of cells (Frydman et al., 1994; Nimmesgern and Hartl, 1993). In the present study, Fluc-based sensors were used to assess the folding capacity of HeLa cells (Figure 22). Fluc has been used in many different model organisms and cells for various applications (Naylor, 1999). Therefore, we believe that the FlucEGFP based sensors can also be used to measure the chaperone capacity of cells derived from different tissues. This will aid in the comprehensive analysis of cell line specific responses to buffer proteotoxic insults. 
We have used the Fluc variants to study the effects of Hsp90 inhibition on the cellular protein folding homeostasis. Upon Hsp90 inhibition by 17-AAG, both FlucSM-EGFP and FlucDM-EGFP formed inclusions while Fluc-EGFP fluorescence remained diffusely distributed in cells (Figure 29a). Several reasons may account for the aggregation of Fluc mutant proteins under these conditions. It could be due to the direct effect of 17-AAG on the folding machinery of cells. Hsp90 has been shown to co-operate with Hsp70-Hsp40 via the co-chaperones such as HOP in refolding stress-denatured proteins and in preventing misfolded proteins to form aggregates (Hutchison et al., 1994; McClellan et al., 2007; Powers et al., 2008; Schumacher et al., 1996; Walerych et al., 2009; Wegele et al., 2006). Additionally, Hsp90 inhibition has been shown to induce transcription of HSPs by HSF1 mediated activation (Sittler et al., 2001). However, the aggregation of Fluc mutants under these conditions suggests that Hsp90 is absolutely essential for their conformational maintenance. It also indicates that in functional absence of Hsp90, other chaperones cannot overtake its essential functions. This is in line with previous reports that demonstrated that Hsp90 is absolutely important for the refolding of Fluc and cannot be substituted by addition of other chaperones (Schroder et al., 1993; Thulasiraman et al., 1999).

Hsp90, besides folding and refolding its client substrates, also participates in silencing mutations that may arise due to genetic polymorphism, genomic instability, mistranslation, or due to incorporation of amino acid analogs in the proteome (Jarosz and Lindquist, 2010; Queitsch et al., 2002; Rutherford and Lindquist, 1998). Therefore, an indirect effect of Hsp90 inhibition on Fluc-EGFP based sensors could be exposure of these "silent mutations" which might have increased substrate load on the proteostasis machinery. The preoccupation of cellular folding factors, including Hsp90, by these hidden mutations might have therefore resulted in aggregation of metastable Fluc variants.

The results presented in this study demonstrate that Fluc-EGFP-based sensors can report the changes in the folding landscape of cells and therefore can be used to measure the effect of stress on the folding machinery of cells. In the past, RRL systems have been widely used to obtain mechanistic details about the role of various chaperones in refolding of firefly luciferase (Nimmesgern and Hartl, 1993; Schumacher et al., 1996) and to identify small molecules that modify protein folding (Cassel et al., 2012; Galam et al., 2007; Wisen and Gestwicki, 2008). We 
suggest that using a RRL based Fluc assay, it should be possible in the future to obtain detailed information about the interactions of various chaperones during the folding of differentially stabilized Fluc proteins.

\section{VI.3. Assessment of Refolding Capacity of Cells by Fluc Sensors}

In cells, following a proteotoxic stress, misfolded proteins need to be cleared to prevent their accumulation and aggregation. This is achieved by refolding and/or degradation pathways that have been shown to integrate with each other to remove these misfolded proteins (Arndt et al., 2007). In cells, refolding is thermodynamically favorable over protein degradation and the decision between these two pathways may depend on two factors. First, the recognition of damage through specific features of the substrate and second, if chaperone mediated refolding fails, the substrate is directed to the degradation pathway (Arndt et al., 2007; Kettern et al., 2010). This means that surface features on a substrate protein, most likely exposed hydrophobic residues, determine the decision of cells whether to refold or degrade a substrate. A substrate that continues to display its hydrophobic residues for a considerable amount of time is eventually targeted to degradation (Marques et al., 2006). For instance, it has been shown in vivo that Hsp90 together with Hsp70 and other co-chaperones mediate the refolding of heat denatured luciferase. Inhibition of Hsp90-mediated refolding by geldanamycin prevents the dissociation of Hsp90 from luciferase and results in proteolysis (Schneider et al., 1996). Therefore, both the refolding and the degradation pathways compete with each other to restore proteostasis after stress.

In this study, we used Fluc-based sensors to monitor the disaggregation and the refolding capacity of cells during recovery from heat stress. In mammalian cells, the Fluc-based sensors formed cytosolic foci during heat stress that disappeared partially during recovery (Figure 25). Moreover, the renaturation of Fluc-EGFP variants (both enzymatic activity and soluble amount) during recovery in the presence of cycloheximide indicates that resolubilization of aggregates had occurred (Figure 26). Our results are in line with previous studies that heat denatured luciferase can be efficiently refolded in cells (Michels et al., 1997; Pinto et al., 1991; Schneider et al., 1996). Although the presence of a disaggregase, such as Hsp104 (in S. cerevisiae) or ClpB (in E. coli), in mammalian cells is still unclear, the resolubilization of Fluc aggregates suggests that a disaggregation activity is present. Consistent with this hypothesis, a recent study identified Hsp110 (Apg-2) as a mammalian disaggregase (Shorter, 2011). However, this study utilized a 
cell free system and therefore the exact mechanism of Hsp110 and its protein interacting partners, if any, is still elusive. We believe that experiments such as immunoprecipitation of Fluc aggregates followed by mass spectrometry will aid in the identification of novel proteins that participate in the disaggregation and the refolding process.

\section{VI.4. Fluc-Based Sensors Report on Tissue-Specific Differences in C. elegans}

An intriguing aspect of protein conformational diseases is the selective vulnerability of some cells and tissues to the toxic effects of disease-associated proteins such as the huntingtin. This specificity occurs despite the ubiquitous expression of these proteins in a wide range of cells and tissues at different developmental stages. This may be explained by variations in the expression levels of chaperones and other components of the PN within different cell types (Powers et al., 2009; Vos et al., 2008). However, a comprehensive understanding of PN regulation during development and aging is still lacking.

Using C. elegans as a model, we observed a difference between muscle and neuronal cells in their capacity to remove FlucDM aggregates during recovery from heat stress (Figure 33). We observed that neuronal cells can recover much faster than muscle cells from acute heat stress. This suggests that the proteostasis machinery in neuronal cells is better equipped to dispose of aggregated proteins. This observation is in agreement with a previous report which states that the chaperoning capacity of body-wall muscle cells is different from that of neuronal cells (Kern et al., 2010). Interestingly, we also observed differences between the muscle and the neuronal cells in their ability to maintain protein homeostasis during aging (Figure 35). We observed that FlucDM in muscle cells appeared as distinct aggregates at day 15 compared to neuronal cells where aggregates could already be detected at day 12 . This suggests that neuronal cells, which are more efficient than muscle cells in recovering from heat stress, are less capable of maintaining proteostasis during chronic proteome stress (e.g. aging in our study).

The tissue-specific differences in maintaining protein stability can be due to multiple factors. One possible explanation are differences in the ability of tissues to mount a HSF1 mediated adaptive stress response. It has been shown previously that neuronal cells are less efficient in mounting a stress response when compared with muscle cells and consequently they are more sensitive to stress (Kern et al., 2010). The molecular basis for this observation is still unclear. Future experiments should be designed that can determine HSF1 expression levels, its 
efficiency to trimerize and to bind promoter and its ability to induce expression of HSPs in muscle and neuronal cells. In C. elegans, even within neuronal cells, differences in protein quality control mechanisms have been observed in the presence of pathogenic polyQ proteins (Brignull et al., 2006). In this study, the authors have shown that polyQ proteins can exist in a soluble state in certain lateral neurons whereas in an aggregated state in motor neurons of the same animal. Therefore it seems possible that different tissues have distinct requirements for certain factors (involved in protein quality control) whose levels can vary significantly. This may then influence their overall buffering capacity.

The tissue-specific differences can also be due to other reasons such as differences in the capacity of clearance pathways, gene-expression profiles, signaling pathways etc. For example, it has been shown recently in mammalian cell lines that muscle cells are more efficient in clearing aggregates of misfolded mutant SOD1 than neuronal cells. This is due to more efficient proteasome activity and rapid activation of the autophagy system in muscle cells (Onesto et al., 2011). Moreover, many reports have demonstrated that during aging there is a decline in the expression and/or activity of the components of the PN such as chaperones (Bonelli et al., 1999; Gutsmann-Conrad et al., 1998; Pahlavani et al., 1995), the UPS (Ferrington et al., 2005; Tonoki et al., 2009) and the autophagy system (Cuervo and Dice, 2000). Therefore, spatial and temporal regulation of the components of the PN may vary in muscle and neuronal cells. To our knowledge, there is presently no data allowing a comparison of the levels of different components of the PN in young and old muscle cells and neuronal cells. We believe that a proteomic analysis of muscle and neuronal cells at different stages of development will further broaden the understanding of regulation and interaction of various components of the PN during aging.

\section{VI.5. Fluc-Based Sensors Report on Proteostasis Collapse by Mutant Huntingtin}

In protein conformational diseases, including $\mathrm{HD}, \mathrm{PD}$ and $\mathrm{AD}$, many questions still remain unanswered such as elucidating the mechanisms by which aggregates and/or oligomeric species cause cellular toxicity, the molecular processes that are affected, tissue-specific differences in response to aggregation and the role of the genetic background. In this study, we used mutant $\mathrm{Htt}$ as a model for an aggregation prone protein and studied its effect on the stability 
of Fluc variants (Figure 30). The severe aggregation of FlucDM, and to a lesser extent that of FlucSM and Fluc, suggests that the protein quality control machinery is compromised by mutant Htt. Our observation supports previous findings where mutant $\mathrm{Htt}$ has been proposed to modulate the folding capacity of cells (Gidalevitz et al., 2006; Satyal et al., 2000). A study by Vendruscolo and co-workers has indicated that proteins are only marginally stable under a given set of conditions where they remain soluble and function efficiently. However these proteins have almost no margin of safety to respond to conditions that impair the cellular regulatory processes (Tartaglia et al., 2007). Therefore, it is likely that Htt induces changes in the proteostasis capacity of cells. This then has the consequence that proteins with a very low margin of stability, like FlucDM, collapse to their unfolded or misfolded conformation.

If the above mentioned hypothesis is true, the next question that arises is which species of Htt inflicts the damage to the proteostasis system? At the moment this question is difficult to answer. While several lines of indirect evidence support a role of Htt inclusion bodies in cellular toxicity (Davies et al., 1997; DiFiglia et al., 1997; Yang et al., 2002), other studies indicate a role of misfolded monomers or oligomers in neuronal cell death (Arrasate et al., 2004; Bodner et al., 2006; Miller et al., 2010; Saudou et al., 1998; Takahashi et al., 2008). To address this question using Fluc sensors, we performed live-cell imaging in HEK 293T cells that were transiently cotransfected with equal amounts of DNA encoding Fluc sensors and mutant Htt (Figure 31). Aggregation of FlucDM-EGFP could be observed earlier than aggregation of Htt97Q-mCherry (Figure 31b). This suggests that an imbalance in proteostasis had occurred before the detection of visible Htt aggregates. This hints towards a role of oligomers in proteotoxicity. However, the detection of Htt oligomers is technically difficult and it is even more challenging to predict the conformation of oligomers that can result in toxicity. It has been reported that Htt can misfold into distinct conformations which exhibit different toxicity levels in cells (Nekooki-Machida et al., 2009).We also observed in our experiments that at later time points, the aggregation of mutant $\mathrm{Htt}$ further accelerated the aggregation of FlucDM-EGFP.

Our two observations: (i) the formation of FlucDM-EGFP aggregates before mutant Htt aggregates and (ii) severe aggregation of FlucDM-EGFP after Htt aggregation has occurred, suggest that both oligomers and visible aggregates of mutant Htt disturb the cellular proteostasis machinery. The fact that FlucDM aggregates and Htt aggregates form at different sites suggests 
that cells may utilize different aggregation pathways for FlucDM and mutant $\mathrm{Htt}$ and deposit aggregates of these proteins to distinct cellular sites such as IPOD (Insoluble Protein Deposit) and JUNQ (Juxta Nuclear Quality control) (Kaganovich et al., 2008). Therefore, by using an inducible expression system it would be interesting to know whether the aggregation of these two proteins is reversible. Through proteomic studies it may then be possible to identify cellular factors responsible for resolubilization and disaggregation.

Several studies have documented a role of Htt in causing UPS impairment (Bence et al., 2001; Bennett et al., 2005; Venkatraman et al., 2004). More recently, it was shown that mutant $\mathrm{Htt}$, whether aggregated or not, does not impair the $26 \mathrm{~S}$ proteasome directly but it leads to the accumulation of ubiquitinated substrates which disrupts cellular proteostasis (Hipp et al., 2012). This study also proposes that $\mathrm{Htt}$ can increase the burden on the folding machinery of cells which causes some normal chaperone substrates to misfold. As a result, Htt may indirectly affect UPS mediated degradation by enhancing the competition among ubiquitinated normal chaperone clients. In our study, we have also observed the severe aggregation of FlucDM-EGFP upon treatment with proteasome inhibitor MG132 (Figure 29a). Therefore, it is likely that besides compromising protein folding homeostasis, Htt also blocks degradation of other cellular proteins leading to global dysfunction of the proteostasis machinery.

\section{VI.6. Analysis of Intra-Compartmental Proteostasis Capacity by Fluc-Based Sensors}

Higher eukaryotes contain over 60 different HSP genes that code for proteins belonging to different families. For example, the human genome encodes 13 different Hsp70 members and 41 different Hsp40 (DnaJ) members which might have resulted from multiple duplications and retrotranspositions during evolution (Brocchieri et al., 2008; Qiu et al., 2006). Under normal conditions, most of the members belonging to these chaperone families are found both in the cytosol and to a lesser extent in the nucleus. The different members within the same chaperone family sometimes share a significant sequence homology such as cytosolic Hsc70 and Hsp70 and sometimes they have a substantial sequence divergence in certain domains, such as DnaJA1 and DnaJB1. However, why the human genome encodes so many members within the same chaperone family is still unclear. On the one hand the redundancy may indicate the requirement of chaperones in different compartments to mediate protein folding. On the other hand, it also 
reflects that chaperones are not generally promiscuous towards their substrates and that cells also require highly specialized chaperones for distinct functions (Hageman et al., 2011; Kabani and Martineau, 2008). Moreover, it has been shown in a recent study that some HSPs exhibit tissueor development specific expression which further makes the folding environment of cells highly dynamic (Hageman and Kampinga, 2009). Therefore, it is reasonable to envision that proteostasis capacity in different compartments could vary and spatial-temporal regulation of the PN may differentially affect the sensitivity of compartments to stress.

In eukaryotic cells there exists a substantial difference between the stability of proteins in the nucleus and in the cytosol. In our study, we observed that both the nucleus and the cytosol have similar proteostasis capacity to keep proteins soluble under normal conditions (Figure 38). However, the severe aggregation of NLS-Fluc-EGFP variants in the nucleus upon heat stress and proteasome inhibition, compared to NES-Fluc-EGFP variants in the cytosol (Figure 40 and 44) demonstrates that under stress conditions, nucleus is more sensitive than the cytosol. This could be due to two reasons; either the nuclear environment is highly dense and crowded with macromolecules which enhances the sensitivity of the Fluc variants to stress and/or, this can be due to the differences in the proteostasis capacity to deal with unfolded proteins. Indeed, it has been shown in past by many studies that the nuclear proteome is highly sensitive to stress and that nuclear damage following protein misfolding stress plays a very critical role in stress induced cell death (Hageman et al., 2007; Michels et al., 1997; Michels et al., 1995).

Under normal growth conditions, the nucleus has a relatively low abundance of molecular chaperones compared to the cytosol. It has been proposed that high concentration of chaperones in the nucleus may be detrimental as they have a high nucleic acid binding affinity which can interfere with essential cellular processes such as DNA synthesis (Velazquez and Lindquist, 1984). However upon stress, many HSPs along with their co-chaperones translocate within $15 \mathrm{~min}$ to the nucleus to prevent protein damage (Anderson et al., 2010; Ellis, 2001; Ohtsuka and Laszlo, 1992; Velazquez and Lindquist, 1984). Although no scientific data exist to explain the lower levels of chaperones in the nucleus, the aggregation of proteins could be a combined effect of macromolecular crowding and the absence of chaperones during the first few minutes of stress. 


\section{VI.7. Role of the Nucleus in Protein Quality Control of Cytoplasmic Proteins}

It is now becoming clearer that cells have evolved a defense mechanism to sequester toxic misfolded proteins to discrete cellular sites; this may depend on the ubiquitination status and aggregation state of these proteins (Kaganovich et al., 2008). Compartmentalization seems to be an ingenious way of sequestering deleterious protein conformers that cannot be degraded immediately. It is known that inhibition of the UPS by MG132 results in the formation of inclusion bodies, also called aggresomes at distinct juxtanuclear location (Johnston et al., 1998; Kopito, 2000). The formation of aggresomes has been proposed to facilitate the efficient clearance of cytoplasmic aggregates by autophagy during UPS impairment (Iwata et al., 2005). Additionally, in yeast and mammalian cells, two additional compartments called JUNQ and IPOD have been reported to sequester cytosolic proteins into distinct compartments (Kaganovich et al., 2008). While the JUNQ is reported to accumulate soluble misfolded proteins near the nucleus, the IPOD sequesters terminally-misfolded insoluble species at the cell's periphery. The proteins in the JUNQ are ubiquitinated and co-localize with proteasomes, whereas the proteins in IPOD are not ubiquitinated and co-localize with the autophagy markers.

In this study, a surprising observation was noticed in the cellular localization of aggregates of NES-Fluc-EGFP variants upon proteasome inhibition (Figure 40). While the aggregates of NES-Fluc-EGFP were mainly cytoplasmic, the aggregates of NES-FlucDM-EGFP were mainly localized in the nucleus. The localization of NES-Fluc-EGFP aggregates near the nucleus suggests that deposition occured either in aggresomes or JUNQ compartments. The livecell imaging experiments showed that upon MG132 stress, aggregates of NES-FlucDM-EGFP formed first in the cytosol and only later began to appear in the nucleus where they gradually increased both in size and number (Figure 41). The gradual concentration of the protein in the nucleus could be due to uncontrolled flux of proteins into the nucleus or due to a reduced activity of the nuclear export machinery. Despite the high concentration of NES-FlucDM-EGFP in the cytosol, its propensity to form aggregates in the nucleus upon proteasome inhibition suggests an additional pathway utilized by cells for protein quality control.

In the nucleus, autophagy is absent (Iwata et al., 2005) and therefore the only known degradation machinery that exist is the UPS. Interestingly, it was demonstrated in S. cerevisiae that the terminally misfolded cytosolic protein, mutant carboxypeptidase $\mathrm{Y}$ without signal 
sequence $\left(\triangle \mathrm{ssCPY}^{*}\right)$, migrates into the nucleus and is selectively ubiquitinated by a nuclear E3 ligase called San1p for degradation by the UPS (Prasad et al., 2010). It is therefore possible that some destabilized proteins in the cytoplasm are recognized and selectively targeted to the nucleus for UPS mediated degradation. Our microscopy results showed the co-localization of NES-FlucDM-EGFP with the 20S proteasome core particle and ubiquitin (Figure 45 and 46), suggesting a role of nuclear factors in the degradation of NES-FlucDM-EGFP. These observations were further supported by our finding that degradation of NES-FlucDM-EGFP was significantly enhanced when its export from the nucleus was blocked by leptomycin B (LMB), an inhibitor of the nuclear export cycle (Figure 47b, c).

Although a mammalian homolog of San1p has not been identified so far, another nuclear E3 ligase called UHRF-2 has been identified as a San1p functional ortholog in mammals (Iwata et al., 2009). Moreover, the over-expression of San1p or UHRF-2 has been shown to enhance degradation of polyQ aggregates in cultured cells and primary neurons (Iwata et al., 2009) which underscores the role of the nucleus in preventing the accumulation of misfolded proteins. Recently, it was also demonstrated that San1p recognizes exposed hydrophobic residues in its substrates for ubiquitination and subsequently targets them for degradation (Fredrickson et al., 2011). Although the molecular mechanism are still far from clear, it seems possible that NESFlucDM-EGFP, due to its structural instability, may expose otherwise buried hydrophobic residues which are eventually recognized by nuclear E3 ligases for degradation. This hypothesis is augmented by the observation that NES-Fluc-EGFP, which is conformationally stable, predominantly forms aggregates in the cytosol upon proteasome inhibition (Figure 40). Reverse genetic screens to identify potential E3 ligases in nucleus for degradation of cytoplasmic proteins will be useful in understanding protein quality control mechanisms in the nucleus.

\section{VI.8. Role of DnaJB1 in the Degradation of Proteins}

The co-localization of NES-FlucDM-EGFP with the 20S proteasome core particle and ubiquitin in the nucleus upon proteasome inhibition (Figure 45 and 46) and its rapid degradation upon addition of the nuclear export inhibitor (LMB) (Figure 47b, c) suggests that under normal conditions this protein may be degraded in the nucleus. In order to be degraded in the nucleus, a cytosolic protein such as NES-FlucDM-EGFP should be selectively recognized and shuttled by some specific cellular factors from the cytosol to the nucleus. Among the components of the 
protein quality control machinery, only molecular chaperones (with co-chaperones) and E3 ubiquitin ligases are known to specifically recognize non-native protein conformations.

Post-translational modifications of proteins like covalent attachment of SUMO (Small Ubiquitin like Modifier) are known to alter the subcellular distribution of the modified protein (Johnson, 2004). SUMO has a similar structure as ubiquitin and it is covalently conjugated to proteins (on Lys residues) by an enzyme cascade in a manner similar to the ubiquitin-conjugation pathway. SUMO E3 ligases recognize a $\Psi$ KXE motif in proteins, where $\Psi$ stands for a large hydrophobic amino acid like Ile, Leu or Val; $\mathrm{K}$ is the Lys residue that is modified; $\mathrm{X}$ is any amino acid and E is a Glu residue (Johnson, 2004; Teng et al., 2012). Interestingly, a study has shown that sumoylation of the Htt protein can alter its stability and promotes neurodegeneration in D. melanogaster (Steffan et al., 2004). Additionally, it has been shown that upon proteasome inhibition, sumoylation of proteins increases and SUMO-conjugated proteins accumulate in the nucleolus (Matafora et al., 2009). Sumoylation of proteins has also been shown to play a role in shuttling of proteins between the cytosol and the nucleus (Johnson, 2004). This made sumoylation a potential mechanism for the transport of misfolded luciferase into the nucleus. However, when we checked the presence of sumoylation sites (YKXE motif) in NES-FlucDMEGFP using the seeSUMO web server (http://bioinfo.ggc.org/seesumo/) (Teng et al., 2012), we could not find any putative sumoylation sites in NES-FlucDM-EGFP. This suggests that nuclear targeting of this protein upon proteasome inhibition does not occur because of sumoylation but may involve other specific sorting factors that recognize and shuttle NES-FlucDM-EGFP to the nucleus.

It has been demonstrated in different cell-types that most of the HSPs such as Hsp90, Hsp70 and Hsp40 immediately move to the nucleus during stress, presumably to prevent the further aggregation of nuclear proteins and to rapidly restore the proteostasis in the nucleus for cell survival (Akner et al., 1992; Kampinga, 1993; Velazquez and Lindquist, 1984; Welch and Feramisco, 1984). Therefore, it is possible that during stress, misfolded proteins bound to HSPs may enter the nucleus via translocation of these HSPs.

Among the various chaperones, the Hsp70-Hsp40 system is extensively studied in terms of both structural and mechanistic details. Hsp40 (DnaJ), besides stimulating ATPase activity of Hsp70 (Cyr et al., 1992; Mayer, 2010), also recognizes, binds and carries substrates to their 
partner Hsp70 molecules (Misselwitz et al., 1998; Rudiger et al., 2001; Szabo et al., 1996). The human genome encodes 41 different Hsp40 members. It has been suggested that different Hsp40s, due to their substrate recognition ability, may provide substrate specificity to Hsp70 (Qiu et al., 2006). The Hsp40 members have in common a conserved J-domain and are subdivided into three distinct groups (DnaJA, DnaJB and DnaJC) based on the presence of certain structural motifs (Cheetham and Caplan, 1998; Kampinga and Craig, 2010). However, among these different Hsp40 members, only DnaJB1 has been studied in detail. In HEK 293T cells, DnaJB1 is moderately expressed and is highly inducible upon heat stress (Hageman et al., 2010; Hageman et al., 2011). It has been shown that DnaJB1 suppresses the aggregation of polyQ proteins (Rujano et al., 2007; Zijlstra et al., 2010) and also prevents luciferase aggregation during heat stress (Hageman et al., 2010). Moreover, it has been demonstrated that overexpression of Hsp70 and DnaJB1 facilitates the degradation of polyQ expanded forms of the androgen receptor (Bailey et al., 2002). Recently, it has also been shown that less characterized members of the DnaJB family, DnaJB6 and DnaJB8, bind non-foldable clients such as polyQ proteins and keep them in a state competent for (proteasomal) degradation (Hageman et al., 2010; Kampinga and Craig, 2010). This suggests that DnaJ proteins, besides participating in folding also play an important role in preventing the accumulation of misfolded proteins by targeting them for proteasome mediated degradation.

These characteristics of DnaJB1 in suppressing protein aggregation and its role in degradation made it a likely candidate to test its effect on the degradation kinetics of NESFlucDM-EGFP. We found that, under conditions when it is retained in the nucleus by the nuclear export inhibitor LMB, degradation of NES-FlucDM-EGFP is significantly reduced when DnaJB1 is down-regulated (Figure 48). Moreover, in cells with reduced DnaJB1, NES-FlucDMEGFP formed inclusions predominantly in the cytosol rather than in the nucleus upon proteasome inhibition (Figure 49). Taken together, these results suggest that DnaJB1 participates in the degradation of misfolded NES-FlucDM-EGFP and may transport misfolded proteins from the cytosol to the nucleus for degradation.

It is interesting to note that DnaJB1 is active as a dimer and has a V-shaped clamp-like architecture that can bind to a large exposed surface area on its substrates with a very high affinity (Stirling et al., 2006). Therefore, it is reasonable to assume that the increase in the 
concentration of DnaJB1, induced by stress, shifts the equilibrium of DnaJB1 from monomers to the dimer formation. DnaJB1 dimers can then efficiently sequester misfolded proteins and carry them to the nucleus during stress. E3 ligases in the nucleus such as San1p or UHRF-2 can then ubiquitinate these proteins and thereby tag them for degradation by the proteasome. This suggests that a protein quality control mechanism for the clearance (sequestration or degradation) of cytosolic misfolded proteins may exist in the nucleus. This mechanism may be more efficient than the mechanisms in the cytosol. More recently, a study has identified two proteins in $S$. cerevisiae called Btn2 and Cur1, which form a complex with Sis1 (DnaJB1 homolog in yeast) during acute stress and transport it to the nucleus (Malinovska et al., 2012). Thus, it is likely that DnaJB1 in higher eukaryotes may also require some sorting factors to shuttle cytosolic misfolded proteins to the nucleus.

In this study, we selected three Fluc variants; the most stable Fluc wild-type, a moderately stable FlucSM and the least stable FlucDM to serve as sensors of proteostasis. We showed that these Fluc variants have different stabilities. These sensors can act as representative examples of both the stable and the metastable proteome of cells. Using these Fluc sensors we can evaluate the effect of proteotoxic stresses on the stability of the endogenous metastable proteome. The differential aggregation propensity (or solubility) and luminescence activity of our sensors measures the status of the PN under different stress conditions. Our results show that Fluc-EGFP based sensors are highly versatile in reporting imbalances in proteostasis both during acute (such as heat stress) and chronic stress (such as expression of mutant Htt and during aging) conditions. This set of three sensors proteins can therefore serve as a convenient toolkit to measure the status of proteostasis in a wide range of experimental systems. Our results also point towards a role of the nucleus in the quality control of misfolded proteins in the cytosol. It will be interesting to obtain mechanistic details about the role of various PN components in maintaining and restoring proteostasis in different cellular compartments. Reporter cell lines simultaneously expressing two or more of these sensors, carrying different fluorescent tags, may be employed to directly compare the fate of differentially destabilized proteins. A major challenge of future research will be to determine the extent to which these sensor proteins can monitor the full range of proteostasis-related functions. Fluc sensors are non-endogenous proteins and they represent a single structural class of proteins. A comparison of their behavior with that of endogenous proteins of different structural classes will be useful for understanding of the PN. 


\section{References}

Abravaya, K., Myers, M.P., Murphy, S.P., and Morimoto, R.I. (1992). The human heat shock protein hsp70 interacts with HSF, the transcription factor that regulates heat shock gene expression. Genes Dev 6, 1153-1164.

Abravaya, K., Phillips, B., and Morimoto, R.I. (1991). Heat shock-induced interactions of heat shock transcription factor and the human hsp70 promoter examined by in vivo footprinting. Mol Cell Biol 11, 586-592.

Agashe, V.R., Guha, S., Chang, H.C., Genevaux, P., Hayer-Hartl, M., Stemp, M., Georgopoulos, C., Hartl, F.U., and Barral, J.M. (2004). Function of trigger factor and DnaK in multidomain protein folding: increase in yield at the expense of folding speed. Cell 117, 199-209.

Akerfelt, M., Morimoto, R.I., and Sistonen, L. (2010). Heat shock factors: integrators of cell stress, development and lifespan. Nat Rev Mol Cell Biol 11, 545-555.

Akerfelt, M., Trouillet, D., Mezger, V., and Sistonen, L. (2007). Heat shock factors at a crossroad between stress and development. Ann N Y Acad Sci 1113, 15-27.

Akner, G., Mossberg, K., Sundqvist, K.G., Gustafsson, J.A., and Wikstrom, A.C. (1992). Evidence for reversible, non-microtubule and non-microfilament-dependent nuclear translocation of hsp90 after heat shock in human fibroblasts. Eur J Cell Biol 58, 356-364.

Ali, A., Bharadwaj, S., O'Carroll, R., and Ovsenek, N. (1998). HSP90 interacts with and regulates the activity of heat shock factor 1 in Xenopus oocytes. Mol Cell Biol 18, 4949-4960.

Amaral, M.D. (2005). Processing of CFTR: traversing the cellular maze--how much CFTR needs to go through to avoid cystic fibrosis? Pediatr Pulmonol 39, 479-491.

Amin, J., Ananthan, J., and Voellmy, R. (1988). Key features of heat shock regulatory elements. Mol Cell Biol 8, 3761-3769.

Ananthan, J., Goldberg, A.L., and Voellmy, R. (1986). Abnormal proteins serve as eukaryotic stress signals and trigger the activation of heat shock genes. Science 232, 522-524.

Anderson, L.G., Meeker, R.B., Poulton, W.E., and Huang, D.Y. (2010). Brain distribution of carboxy terminus of Hsc70-interacting protein (CHIP) and its nuclear translocation in cultured cortical neurons following heat stress or oxygen-glucose deprivation. Cell Stress Chaperones 15, 487-495.

Arndt, V., Rogon, C., and Hohfeld, J. (2007). To be, or not to be--molecular chaperones in protein degradation. Cell Mol Life Sci 64, 2525-2541.

Arrasate, M., Mitra, S., Schweitzer, E.S., Segal, M.R., and Finkbeiner, S. (2004). Inclusion body formation reduces levels of mutant huntingtin and the risk of neuronal death. Nature 431, 805-810.

Audas, T.E., Jacob, M.D., and Lee, S. (2012). Immobilization of proteins in the nucleolus by ribosomal intergenic spacer noncoding RNA. Mol Cell 45, 147-157. 
Auluck, P.K., Chan, H.Y., Trojanowski, J.Q., Lee, V.M., and Bonini, N.M. (2002). Chaperone suppression of alpha-synuclein toxicity in a Drosophila model for Parkinson's disease. Science 295, 865868.

Bailey, C.K., Andriola, I.F., Kampinga, H.H., and Merry, D.E. (2002). Molecular chaperones enhance the degradation of expanded polyglutamine repeat androgen receptor in a cellular model of spinal and bulbar muscular atrophy. Hum Mol Genet 11, 515-523.

Balch, W.E., Morimoto, R.I., Dillin, A., and Kelly, J.W. (2008). Adapting proteostasis for disease intervention. Science 319, 916-919.

Baldwin, R.L. (1989). How does protein folding get started? Trends Biochem Sci 14, 291-294.

Baler, R., Dahl, G., and Voellmy, R. (1993). Activation of human heat shock genes is accompanied by oligomerization, modification, and rapid translocation of heat shock transcription factor HSF1. Mol Cell Biol 13, 2486-2496.

Baler, R., Welch, W.J., and Voellmy, R. (1992). Heat shock gene regulation by nascent polypeptides and denatured proteins: hsp70 as a potential autoregulatory factor. J Cell Biol 117, 1151-1159.

Ballinger, C.A., Connell, P., Wu, Y., Hu, Z., Thompson, L.J., Yin, L.Y., and Patterson, C. (1999). Identification of CHIP, a novel tetratricopeptide repeat-containing protein that interacts with heat shock proteins and negatively regulates chaperone functions. Mol Cell Biol 19, 4535-4545.

Banski, P., Kodiha, M., and Stochaj, U. (2010). Chaperones and multitasking proteins in the nucleolus: networking together for survival? Trends Biochem Sci 35, 361-367.

Bardwell, J.C., and Craig, E.A. (1988). Ancient heat shock gene is dispensable. J Bacteriol 170, 29772983.

Baumeister, W., Walz, J., Zuhl, F., and Seemuller, E. (1998). The proteasome: paradigm of a selfcompartmentalizing protease. Cell 92, 367-380.

Behrends, C., Langer, C.A., Boteva, R., Bottcher, U.M., Stemp, M.J., Schaffar, G., Rao, B.V., Giese, A., Kretzschmar, H., Siegers, K., et al. (2006). Chaperonin TRiC promotes the assembly of polyQ expansion proteins into nontoxic oligomers. Mol Cell 23, 887-897.

Ben-Zvi, A., De Los Rios, P., Dietler, G., and Goloubinoff, P. (2004). Active solubilization and refolding of stable protein aggregates by cooperative unfolding action of individual hsp70 chaperones. J Biol Chem 279, 37298-37303.

Ben-Zvi, A., Miller, E.A., and Morimoto, R.I. (2009). Collapse of proteostasis represents an early molecular event in Caenorhabditis elegans aging. Proc Natl Acad Sci U S A 106, 14914-14919.

Ben-Zvi, A.P., and Goloubinoff, P. (2001). Review: mechanisms of disaggregation and refolding of stable protein aggregates by molecular chaperones. J Struct Biol 135, 84-93.

Bence, N.F., Sampat, R.M., and Kopito, R.R. (2001). Impairment of the ubiquitin-proteasome system by protein aggregation. Science 292, 1552-1555. 
Bennett, E.J., Bence, N.F., Jayakumar, R., and Kopito, R.R. (2005). Global impairment of the ubiquitinproteasome system by nuclear or cytoplasmic protein aggregates precedes inclusion body formation. Mol Cell 17, 351-365.

Bercovich, B., Stancovski, I., Mayer, A., Blumenfeld, N., Laszlo, A., Schwartz, A.L., and Ciechanover, A. (1997). Ubiquitin-dependent degradation of certain protein substrates in vitro requires the molecular chaperone Hsc70. J Biol Chem 272, 9002-9010.

Bienz, M., and Pelham, H.R. (1987). Mechanisms of heat-shock gene activation in higher eukaryotes. Adv Genet 24, 31-72.

Blond-Elguindi, S., Cwirla, S.E., Dower, W.J., Lipshutz, R.J., Sprang, S.R., Sambrook, J.F., and Gething, M.J. (1993). Affinity panning of a library of peptides displayed on bacteriophages reveals the binding specificity of BiP. Cell 75, 717-728.

Bloss, T.A., Witze, E.S., and Rothman, J.H. (2003). Suppression of CED-3-independent apoptosis by mitochondrial betaNAC in Caenorhabditis elegans. Nature 424, 1066-1071.

Bodner, R.A., Outeiro, T.F., Altmann, S., Maxwell, M.M., Cho, S.H., Hyman, B.T., McLean, P.J., Young, A.B., Housman, D.E., and Kazantsev, A.G. (2006). Pharmacological promotion of inclusion formation: a therapeutic approach for Huntington's and Parkinson's diseases. Proc Natl Acad Sci U S A $103,4246-4251$.

Boisvert, F.M., van Koningsbruggen, S., Navascues, J., and Lamond, A.I. (2007). The multifunctional nucleolus. Nat Rev Mol Cell Biol 8, 574-585.

Bonelli, M.A., Alfieri, R.R., Petronini, P.G., Brigotti, M., Campanini, C., and Borghetti, A.F. (1999). Attenuated expression of 70-kDa heat shock protein in WI-38 human fibroblasts during aging in vitro. Exp Cell Res 252, 20-32.

Borkovich, K.A., Farrelly, F.W., Finkelstein, D.B., Taulien, J., and Lindquist, S. (1989). hsp82 is an essential protein that is required in higher concentrations for growth of cells at higher temperatures. Mol Cell Biol 9, 3919-3930.

Boulon, S., Westman, B.J., Hutten, S., Boisvert, F.M., and Lamond, A.I. (2010). The nucleolus under stress. Mol Cell 40, 216-227.

Boyd, M.T., Vlatkovic, N., and Rubbi, C.P. (2011). The nucleolus directly regulates p53 export and degradation. J Cell Biol 194, 689-703.

Bradford, M.M. (1976). A rapid and sensitive method for the quantitation of microgram quantities of protein utilizing the principle of protein-dye binding. Anal Biochem 72, 248-254.

Braig, K., Otwinowski, Z., Hegde, R., Boisvert, D.C., Joachimiak, A., Horwich, A.L., and Sigler, P.B. (1994). The crystal structure of the bacterial chaperonin GroEL at 2.8 A. Nature 371, 578-586.

Brandt, F., Carlson, L.A., Hartl, F.U., Baumeister, W., and Grunewald, K. (2010). The three-dimensional organization of polyribosomes in intact human cells. Mol Cell 39, 560-569.

Brandt, F., Etchells, S.A., Ortiz, J.O., Elcock, A.H., Hartl, F.U., and Baumeister, W. (2009). The native 3D organization of bacterial polysomes. Cell 136, 261-271. 
Braun, B.C., Glickman, M., Kraft, R., Dahlmann, B., Kloetzel, P.M., Finley, D., and Schmidt, M. (1999). The base of the proteasome regulatory particle exhibits chaperone-like activity. Nat Cell Biol 1, 221-226.

Brignull, H.R., Moore, F.E., Tang, S.J., and Morimoto, R.I. (2006). Polyglutamine proteins at the pathogenic threshold display neuron-specific aggregation in a pan-neuronal Caenorhabditis elegans model. J Neurosci 26, 7597-7606.

Broadley, S.A., and Hartl, F.U. (2009). The role of molecular chaperones in human misfolding diseases. FEBS Lett 583, 2647-2653.

Brocchieri, L., Conway de Macario, E., and Macario, A.J. (2008). hsp70 genes in the human genome: Conservation and differentiation patterns predict a wide array of overlapping and specialized functions. BMC Evol Biol 8, 19.

Brockwell, D.J., and Radford, S.E. (2007). Intermediates: ubiquitous species on folding energy landscapes? Curr Opin Struct Biol 17, 30-37.

Brugge, J.S., Erikson, E., and Erikson, R.L. (1981). The specific interaction of the Rous sarcoma virus transforming protein, pp60src, with two cellular proteins. Cell 25, 363-372.

Bryngelson, J.D., Onuchic, J.N., Socci, N.D., and Wolynes, P.G. (1995). Funnels, pathways, and the energy landscape of protein folding: a synthesis. Proteins 21, 167-195.

Bucciantini, M., Giannoni, E., Chiti, F., Baroni, F., Formigli, L., Zurdo, J., Taddei, N., Ramponi, G., Dobson, C.M., and Stefani, M. (2002). Inherent toxicity of aggregates implies a common mechanism for protein misfolding diseases. Nature 416, 507-511.

Bukau, B., and Horwich, A.L. (1998). The Hsp70 and Hsp60 chaperone machines. Cell 92, 351-366.

Bush, K.T., Goldberg, A.L., and Nigam, S.K. (1997). Proteasome inhibition leads to a heat-shock response, induction of endoplasmic reticulum chaperones, and thermotolerance. J Biol Chem 272, 90869092.

Calamini, B., Silva, M.C., Madoux, F., Hutt, D.M., Khanna, S., Chalfant, M.A., Saldanha, S.A., Hodder, P., Tait, B.D., Garza, D., et al. (2012). Small-molecule proteostasis regulators for protein conformational diseases. Nat Chem Biol 8, 185-196.

Calloni, G., Chen, T., Schermann, S.M., Chang, H.C., Genevaux, P., Agostini, F., Tartaglia, G.G., Hayer-Hartl, M., Hartl, F.U. (2012). DnaK Functions as a Central Hub in the E. coli Chaperone Network. Cell Reports 1, 251-264.

Cardozo, C. (1993). Catalytic components of the bovine pituitary multicatalytic proteinase complex (proteasome). Enzyme Protein 47, 296-305.

Cassel, J.A., Ilyin, S., McDonnell, M.E., and Reitz, A.B. (2012). Novel inhibitors of heat shock protein Hsp70-mediated luciferase refolding that bind to DnaJ. Bioorg Med Chem 20, 3609-3614.

Cattaneo, E., Zuccato, C., and Tartari, M. (2005). Normal huntingtin function: an alternative approach to Huntington's disease. Nat Rev Neurosci 6, 919-930. 
Chai, Y., Koppenhafer, S.L., Shoesmith, S.J., Perez, M.K., and Paulson, H.L. (1999). Evidence for proteasome involvement in polyglutamine disease: localization to nuclear inclusions in SCA3/MJD and suppression of polyglutamine aggregation in vitro. Hum Mol Genet 8, 673-682.

Chai, Y., Shao, J., Miller, V.M., Williams, A., and Paulson, H.L. (2002). Live-cell imaging reveals divergent intracellular dynamics of polyglutamine disease proteins and supports a sequestration model of pathogenesis. Proc Natl Acad Sci U S A 99, 9310-9315.

Chakraborty, K., Chatila, M., Sinha, J., Shi, Q., Poschner, B.C., Sikor, M., Jiang, G., Lamb, D.C., Hartl, F.U., and Hayer-Hartl, M. (2010). Chaperonin-catalyzed rescue of kinetically trapped states in protein folding. Cell 142, 112-122.

Cheetham, M.E., and Caplan, A.J. (1998). Structure, function and evolution of DnaJ: conservation and adaptation of chaperone function. Cell Stress Chaperones 3, 28-36.

Chen, B., Zhong, D., and Monteiro, A. (2006). Comparative genomics and evolution of the HSP90 family of genes across all kingdoms of organisms. BMC Genomics 7, 156.

Chen, S., Berthelier, V., Hamilton, J.B., O'Nuallain, B., and Wetzel, R. (2002). Amyloid-like features of polyglutamine aggregates and their assembly kinetics. Biochemistry 41, 7391-7399.

Ciechanover, A. (1998). The ubiquitin-proteasome pathway: on protein death and cell life. EMBO J 17, 7151-7160.

Ciechanover, A. (2005). Proteolysis: from the lysosome to ubiquitin and the proteasome. Nat Rev Mol Cell Biol 6, 79-87.

Cintron, N.S., and Toft, D. (2006). Defining the requirements for Hsp40 and Hsp70 in the Hsp90 chaperone pathway. J Biol Chem 281, 26235-26244.

Clark, P.L. (2004). Protein folding in the cell: reshaping the folding funnel. Trends Biochem Sci 29, 527534.

Cohen, E., Bieschke, J., Perciavalle, R.M., Kelly, J.W., and Dillin, A. (2006). Opposing activities protect against age-onset proteotoxicity. Science 313, 1604-1610.

Cohen, E., Paulsson, J.F., Blinder, P., Burstyn-Cohen, T., Du, D., Estepa, G., Adame, A., Pham, H.M., Holzenberger, M., Kelly, J.W., et al. (2009). Reduced IGF-1 signaling delays age-associated proteotoxicity in mice. Cell 139, 1157-1169.

Connell, P., Ballinger, C.A., Jiang, J., Wu, Y., Thompson, L.J., Hohfeld, J., and Patterson, C. (2001). The co-chaperone CHIP regulates protein triage decisions mediated by heat-shock proteins. Nat Cell Biol 3, 93-96.

Conti, E., Franks, N.P., and Brick, P. (1996). Crystal structure of firefly luciferase throws light on a superfamily of adenylate-forming enzymes. Structure 4, 287-298.

Corrales, F.J., and Fersht, A.R. (1996). Toward a mechanism for GroEL.GroES chaperone activity: an ATPase-gated and -pulsed folding and annealing cage. Proc Natl Acad Sci U S A 93, 4509-4512. 
Csermely, P., and Kahn, C.R. (1991). The 90-kDa heat shock protein (hsp-90) possesses an ATP binding site and autophosphorylating activity. J Biol Chem 266, 4943-4950.

Cuervo, A.M. (2011). Chaperone-mediated autophagy: Dice's 'wild' idea about lysosomal selectivity. Nat Rev Mol Cell Biol 12, 535-541.

Cuervo, A.M., and Dice, J.F. (2000). Age-related decline in chaperone-mediated autophagy. J Biol Chem $275,31505-31513$.

Cuervo, A.M., Knecht, E., Terlecky, S.R., and Dice, J.F. (1995). Activation of a selective pathway of lysosomal proteolysis in rat liver by prolonged starvation. Am J Physiol 269, C1200-1208.

Cuervo, A.M., Stefanis, L., Fredenburg, R., Lansbury, P.T., and Sulzer, D. (2004). Impaired degradation of mutant alpha-synuclein by chaperone-mediated autophagy. Science 305, 1292-1295.

Cunningham, C.N., Krukenberg, K.A., and Agard, D.A. (2008). Intra- and intermonomer interactions are required to synergistically facilitate ATP hydrolysis in Hsp90. J Biol Chem 283, 21170-21178.

Cypser, J.R., Tedesco, P., and Johnson, T.E. (2006). Hormesis and aging in Caenorhabditis elegans. Exp Gerontol 41, 935-939.

Cyr, D.M., Lu, X., and Douglas, M.G. (1992). Regulation of Hsp70 function by a eukaryotic DnaJ homolog. J Biol Chem 267, 20927-20931.

Daggett, V., and Fersht, A.R. (2003). Is there a unifying mechanism for protein folding? Trends Biochem Sci 28, 18-25.

David, D.C., Ollikainen, N., Trinidad, J.C., Cary, M.P., Burlingame, A.L., and Kenyon, C. (2010). Widespread protein aggregation as an inherent part of aging in C. elegans. PLoS Biol 8, e1000450.

Davies, S.W., Turmaine, M., Cozens, B.A., DiFiglia, M., Sharp, A.H., Ross, C.A., Scherzinger, E., Wanker, E.E., Mangiarini, L., and Bates, G.P. (1997). Formation of neuronal intranuclear inclusions underlies the neurological dysfunction in mice transgenic for the HD mutation. Cell 90, 537-548.

Dekker, C., Roe, S.M., McCormack, E.A., Beuron, F., Pearl, L.H., and Willison, K.R. (2011). The crystal structure of yeast CCT reveals intrinsic asymmetry of eukaryotic cytosolic chaperonins. EMBO J 30, 3078-3090.

Deluca, M. (1976). Firefly luciferase. Adv Enzymol Relat Areas Mol Biol 44, 37-68.

Deng, J.M., and Behringer, R.R. (1995). An insertional mutation in the BTF3 transcription factor gene leads to an early postimplantation lethality in mice. Transgenic Res 4, 264-269.

Deuerling, E., Schulze-Specking, A., Tomoyasu, T., Mogk, A., and Bukau, B. (1999). Trigger factor and DnaK cooperate in folding of newly synthesized proteins. Nature 400, 693-696.

Diamant, S., Ben-Zvi, A.P., Bukau, B., and Goloubinoff, P. (2000). Size-dependent disaggregation of stable protein aggregates by the DnaK chaperone machinery. J Biol Chem 275, 21107-21113.

Dias, C.L., Ala-Nissila, T., Wong-ekkabut, J., Vattulainen, I., Grant, M., and Karttunen, M. (2010). The hydrophobic effect and its role in cold denaturation. Cryobiology 60, 91-99. 
DiFiglia, M., Sapp, E., Chase, K.O., Davies, S.W., Bates, G.P., Vonsattel, J.P., and Aronin, N. (1997). Aggregation of huntingtin in neuronal intranuclear inclusions and dystrophic neurites in brain. Science 277, 1990-1993.

Dill, K.A., Bromberg, S., Yue, K., Fiebig, K.M., Yee, D.P., Thomas, P.D., and Chan, H.S. (1995). Principles of protein folding--a perspective from simple exact models. Protein Sci 4, 561-602.

Dill, K.A., and Chan, H.S. (1997). From Levinthal to pathways to funnels. Nat Struct Biol 4, 10-19.

Ditzel, L., Lowe, J., Stock, D., Stetter, K.O., Huber, H., Huber, R., and Steinbacher, S. (1998). Crystal structure of the thermosome, the archaeal chaperonin and homolog of CCT. Cell 93, 125-138.

Dobrzynski, J.K., Sternlicht, M.L., Farr, G.W., and Sternlicht, H. (1996). Newly-synthesized beta-tubulin demonstrates domain-specific interactions with the cytosolic chaperonin. Biochemistry 35, 15870-15882.

Dobson, C.M. (1999). Protein misfolding, evolution and disease. Trends Biochem Sci 24, 329-332.

Dobson, C.M. (2003). Protein folding and misfolding. Nature 426, 884-890.

Douglas, P.M., and Dillin, A. (2010). Protein homeostasis and aging in neurodegeneration. J Cell Biol 190, 719-729.

Doyle, S.M., and Wickner, S. (2009). Hsp104 and ClpB: protein disaggregating machines. Trends Biochem Sci 34, 40-48.

Dragovic, Z., Broadley, S.A., Shomura, Y., Bracher, A., and Hartl, F.U. (2006). Molecular chaperones of the Hsp1 10 family act as nucleotide exchange factors of Hsp70s. EMBO J 25, 2519-2528.

Drummond, D.A., and Wilke, C.O. (2008). Mistranslation-induced protein misfolding as a dominant constraint on coding-sequence evolution. Cell 134, 341-352.

Dunker, A.K., Silman, I., Uversky, V.N., and Sussman, J.L. (2008). Function and structure of inherently disordered proteins. Curr Opin Struct Biol 18, 756-764.

Duyao, M., Ambrose, C., Myers, R., Novelletto, A., Persichetti, F., Frontali, M., Folstein, S., Ross, C., Franz, M., Abbott, M., et al. (1993). Trinucleotide repeat length instability and age of onset in Huntington's disease. Nat Genet 4, 387-392.

Ebeling, W., Hennrich, N., Klockow, M., Metz, H., Orth, H.D., and Lang, H. (1974). Proteinase K from Tritirachium album Limber. Eur J Biochem 47, 91-97.

Ellis, J. (1987). Proteins as molecular chaperones. Nature 328, 378-379.

Ellis, R.J. (1993). The general concept of molecular chaperones. Philos Trans R Soc Lond B Biol Sci 339, 257-261.

Ellis, R.J. (1994). Molecular chaperones. Opening and closing the Anfinsen cage. Curr Biol 4, 633-635.

Ellis, R.J. (2001). Macromolecular crowding: obvious but underappreciated. Trends Biochem Sci 26, 597-604. 
Ellis, R.J. (2006). Molecular chaperones: assisting assembly in addition to folding. Trends Biochem Sci $31,395-401$.

Ewalt, K.L., Hendrick, J.P., Houry, W.A., and Hartl, F.U. (1997). In vivo observation of polypeptide flux through the bacterial chaperonin system. Cell 90, 491-500.

Fargnoli, J., Kunisada, T., Fornace, A.J., Jr., Schneider, E.L., and Holbrook, N.J. (1990). Decreased expression of heat shock protein $70 \mathrm{mRNA}$ and protein after heat treatment in cells of aged rats. Proc Natl Acad Sci U S A 87, 846-850.

Fayet, O., Ziegelhoffer, T., and Georgopoulos, C. (1989). The groES and groEL heat shock gene products of Escherichia coli are essential for bacterial growth at all temperatures. J Bacteriol 171, 1379-1385.

Ferrington, D.A., Husom, A.D., and Thompson, L.V. (2005). Altered proteasome structure, function, and oxidation in aged muscle. FASEB J 19, 644-646.

Fersht, A.R. (2008). From the first protein structures to our current knowledge of protein folding: delights and scepticisms. Nat Rev Mol Cell Biol 9, 650-654.

Finkbeiner, S. (2011). Huntington's Disease. Cold Spring Harb Perspect Biol 3.

Finley, D., Sadis, S., Monia, B.P., Boucher, P., Ecker, D.J., Crooke, S.T., and Chau, V. (1994). Inhibition of proteolysis and cell cycle progression in a multiubiquitination-deficient yeast mutant. Mol Cell Biol 14, 5501-5509.

Fischer-Fantuzzi, L., and Vesco, C. (1988). Cell-dependent efficiency of reiterated nuclear signals in a mutant simian virus 40 oncoprotein targeted to the nucleus. Mol Cell Biol 8, 5495-5503.

Fisher, E.A., Zhou, M., Mitchell, D.M., Wu, X., Omura, S., Wang, H., Goldberg, A.L., and Ginsberg, H.N. (1997). The degradation of apolipoprotein B100 is mediated by the ubiquitin-proteasome pathway and involves heat shock protein 70. J Biol Chem 272, 20427-20434.

Flaherty, K.M., DeLuca-Flaherty, C., and McKay, D.B. (1990). Three-dimensional structure of the ATPase fragment of a 70K heat-shock cognate protein. Nature 346, 623-628.

Flynn, G.C., Pohl, J., Flocco, M.T., and Rothman, J.E. (1991). Peptide-binding specificity of the molecular chaperone BiP. Nature 353, 726-730.

Fornerod, M., Ohno, M., Yoshida, M., and Mattaj, I.W. (1997). CRM1 is an export receptor for leucinerich nuclear export signals. Cell 90, 1051-1060.

Fredrickson, E.K., Rosenbaum, J.C., Locke, M.N., Milac, T.I., and Gardner, R.G. (2011). Exposed hydrophobicity is a key determinant of nuclear quality control degradation. Mol Biol Cell 22, 2384-2395.

Freeman, B.C., and Morimoto, R.I. (1996). The human cytosolic molecular chaperones hsp90, hsp70 (hsc70) and hdj-1 have distinct roles in recognition of a non-native protein and protein refolding. EMBO J $15,2969-2979$.

Frydman, J., Erdjument-Bromage, H., Tempst, P., and Hartl, F.U. (1999). Co-translational domain folding as the structural basis for the rapid de novo folding of firefly luciferase. Nat Struct Biol 6, 697-705. 
Frydman, J., Nimmesgern, E., Erdjument-Bromage, H., Wall, J.S., Tempst, P., and Hartl, F.U. (1992). Function in protein folding of TRiC, a cytosolic ring complex containing TCP-1 and structurally related subunits. EMBO J 11, 4767-4778.

Frydman, J., Nimmesgern, E., Ohtsuka, K., and Hartl, F.U. (1994). Folding of nascent polypeptide chains in a high molecular mass assembly with molecular chaperones. Nature 370, 111-117.

Fukuda, M., Asano, S., Nakamura, T., Adachi, M., Yoshida, M., Yanagida, M., and Nishida, E. (1997). CRM1 is responsible for intracellular transport mediated by the nuclear export signal. Nature 390, 308311.

Galam, L., Hadden, M.K., Ma, Z., Ye, Q.Z., Yun, B.G., Blagg, B.S., and Matts, R.L. (2007). Highthroughput assay for the identification of Hsp90 inhibitors based on Hsp90-dependent refolding of firefly luciferase. Bioorg Med Chem 15, 1939-1946.

Gao, Y., Thomas, J.O., Chow, R.L., Lee, G.H., and Cowan, N.J. (1992). A cytoplasmic chaperonin that catalyzes beta-actin folding. Cell 69, 1043-1050.

Geissler, S., Siegers, K., and Schiebel, E. (1998). A novel protein complex promoting formation of functional alpha- and gamma-tubulin. EMBO J 17, 952-966.

Georgalis, Y., Starikov, E.B., Hollenbach, B., Lurz, R., Scherzinger, E., Saenger, W., Lehrach, H., and Wanker, E.E. (1998). Huntingtin aggregation monitored by dynamic light scattering. Proc Natl Acad Sci U S A 95, 6118-6121.

Georgopoulos, C. (1992). The emergence of the chaperone machines. Trends Biochem Sci 17, 295-299.

Gidalevitz, T., Ben-Zvi, A., Ho, K.H., Brignull, H.R., and Morimoto, R.I. (2006). Progressive disruption of cellular protein folding in models of polyglutamine diseases. Science 311, 1471-1474.

Gidalevitz, T., Kikis, E.A., and Morimoto, R.I. (2010). A cellular perspective on conformational disease: the role of genetic background and proteostasis networks. Curr Opin Struct Biol 20, 23-32.

Gidalevitz, T., Prahlad, V., and Morimoto, R.I. (2011). The stress of protein misfolding: from single cells to multicellular organisms. Cold Spring Harb Perspect Biol 3.

Glover, J.R., and Lindquist, S. (1998). Hsp104, Hsp70, and Hsp40: a novel chaperone system that rescues previously aggregated proteins. Cell $94,73-82$.

Goloubinoff, P., Mogk, A., Zvi, A.P., Tomoyasu, T., and Bukau, B. (1999). Sequential mechanism of solubilization and refolding of stable protein aggregates by a bichaperone network. Proc Natl Acad Sci U S A 96, 13732-13737.

Gould, S.J., Keller, G.A., Hosken, N., Wilkinson, J., and Subramani, S. (1989). A conserved tripeptide sorts proteins to peroxisomes. J Cell Biol 108, 1657-1664.

Groll, M., Ditzel, L., Lowe, J., Stock, D., Bochtler, M., Bartunik, H.D., and Huber, R. (1997). Structure of 20S proteasome from yeast at 2.4 A resolution. Nature 386, 463-471.

Gross, M., and Hessefort, S. (1996). Purification and characterization of a 66-kDa protein from rabbit reticulocyte lysate which promotes the recycling of hsp 70. J Biol Chem 271, 16833-16841. 
Guo, Y., Guettouche, T., Fenna, M., Boellmann, F., Pratt, W.B., Toft, D.O., Smith, D.F., and Voellmy, R. (2001). Evidence for a mechanism of repression of heat shock factor 1 transcriptional activity by a multichaperone complex. J Biol Chem 276, 45791-45799.

Gutsmann-Conrad, A., Heydari, A.R., You, S., and Richardson, A. (1998). The expression of heat shock protein 70 decreases with cellular senescence in vitro and in cells derived from young and old human subjects. Exp Cell Res 241, 404-413.

Hadley, K.C., Borrelli, M.J., Lepock, J.R., McLaurin, J., Croul, S.E., Guha, A., and Chakrabartty, A. (2011). Multiphoton ANS fluorescence microscopy as an in vivo sensor for protein misfolding stress. Cell Stress Chaperones 16, 549-561.

Hageman, J., and Kampinga, H.H. (2009). Computational analysis of the human HSPH/HSPA/DNAJ family and cloning of a human HSPH/HSPA/DNAJ expression library. Cell Stress Chaperones 14, 1-21.

Hageman, J., Rujano, M.A., van Waarde, M.A., Kakkar, V., Dirks, R.P., Govorukhina, N., OosterveldHut, H.M., Lubsen, N.H., and Kampinga, H.H. (2010). A DNAJB chaperone subfamily with HDACdependent activities suppresses toxic protein aggregation. Mol Cell 37, 355-369.

Hageman, J., van Waarde, M.A., Zylicz, A., Walerych, D., and Kampinga, H.H. (2011). The diverse members of the mammalian HSP70 machine show distinct chaperone-like activities. Biochem J 435, 127142.

Hageman, J., Vos, M.J., van Waarde, M.A., and Kampinga, H.H. (2007). Comparison of intra-organellar chaperone capacity for dealing with stress-induced protein unfolding. J Biol Chem 282, 34334-34345.

Hansen, W.J., Cowan, N.J., and Welch, W.J. (1999). Prefoldin-nascent chain complexes in the folding of cytoskeletal proteins. J Cell Biol 145, 265-277.

Hara, T., Nakamura, K., Matsui, M., Yamamoto, A., Nakahara, Y., Suzuki-Migishima, R., Yokoyama, M., Mishima, K., Saito, I., Okano, H., et al. (2006). Suppression of basal autophagy in neural cells causes neurodegenerative disease in mice. Nature 441, 885-889.

Harris, S.F., Shiau, A.K., and Agard, D.A. (2004). The crystal structure of the carboxy-terminal dimerization domain of htpG, the Escherichia coli Hsp90, reveals a potential substrate binding site. Structure 12, 1087-1097.

Harrison, C.J., Hayer-Hartl, M., Di Liberto, M., Hartl, F., and Kuriyan, J. (1997). Crystal structure of the nucleotide exchange factor GrpE bound to the ATPase domain of the molecular chaperone DnaK. Science $276,431-435$.

Hartl, F.U., Bracher, A., and Hayer-Hartl, M. (2011). Molecular chaperones in protein folding and proteostasis. Nature 475, 324-332.

Hartl, F.U., and Hayer-Hartl, M. (2002). Molecular chaperones in the cytosol: from nascent chain to folded protein. Science 295, 1852-1858.

Hartl, F.U., and Hayer-Hartl, M. (2009). Converging concepts of protein folding in vitro and in vivo. Nat Struct Mol Biol 16, 574-581.

Haslbeck, M. (2002). sHsps and their role in the chaperone network. Cell Mol Life Sci 59, 1649-1657. 
Haslbeck, M., Franzmann, T., Weinfurtner, D., and Buchner, J. (2005). Some like it hot: the structure and function of small heat-shock proteins. Nat Struct Mol Biol 12, 842-846.

Hattori, H., Kaneda, T., Lokeshwar, B., Laszlo, A., and Ohtsuka, K. (1993). A stress-inducible 40 kDa protein (hsp40): purification by modified two-dimensional gel electrophoresis and co-localization with hsc70(p73) in heat-shocked HeLa cells. J Cell Sci 104 (3), 629-638.

Hay, D.G., Sathasivam, K., Tobaben, S., Stahl, B., Marber, M., Mestril, R., Mahal, A., Smith, D.L., Woodman, B., and Bates, G.P. (2004). Progressive decrease in chaperone protein levels in a mouse model of Huntington's disease and induction of stress proteins as a therapeutic approach. Hum Mol Genet 13, 1389-1405.

Hemmingsen, S.M., Woolford, C., van der Vies, S.M., Tilly, K., Dennis, D.T., Georgopoulos, C.P., Hendrix, R.W., and Ellis, R.J. (1988). Homologous plant and bacterial proteins chaperone oligomeric protein assembly. Nature 333, 330-334.

Hershko, A., and Ciechanover, A. (1998). The ubiquitin system. Annu Rev Biochem 67, 425-479.

Hessling, M., Richter, K., and Buchner, J. (2009). Dissection of the ATP-induced conformational cycle of the molecular chaperone Hsp90. Nat Struct Mol Biol 16, 287-293.

Hesterkamp, T., Hauser, S., Lutcke, H., and Bukau, B. (1996). Escherichia coli trigger factor is a prolyl isomerase that associates with nascent polypeptide chains. Proc Natl Acad Sci U S A 93, 4437-4441.

Hipp, M.S., Patel, C.N., Bersuker, K., Riley, B.E., Kaiser, S.E., Shaler, T.A., Brandeis, M., and Kopito, R.R. (2012). Indirect inhibition of $26 \mathrm{~S}$ proteasome activity in a cellular model of Huntington's disease. J Cell Biol 196, 573-587.

Hohfeld, J., and Jentsch, S. (1997). GrpE-like regulation of the hsc70 chaperone by the anti-apoptotic protein BAG-1. EMBO J 16, 6209-6216.

Hohfeld, J., Minami, Y., and Hartl, F.U. (1995). Hip, a novel cochaperone involved in the eukaryotic Hsc70/Hsp40 reaction cycle. Cell 83, 589-598.

Horwich, A.L., Low, K.B., Fenton, W.A., Hirshfield, I.N., and Furtak, K. (1993). Folding in vivo of bacterial cytoplasmic proteins: role of GroEL. Cell 74, 909-917.

Houry, W.A., Frishman, D., Eckerskorn, C., Lottspeich, F., and Hartl, F.U. (1999). Identification of in vivo substrates of the chaperonin GroEL. Nature 402, 147-154.

Howarth, J.L., Glover, C.P., and Uney, J.B. (2009). HSP70 interacting protein prevents the accumulation of inclusions in polyglutamine disease. J Neurochem 108, 945-951.

Hsu, A.L., Murphy, C.T., and Kenyon, C. (2003). Regulation of aging and age-related disease by DAF-16 and heat-shock factor. Science 300, 1142-1145.

Hunt, J.F., Weaver, A.J., Landry, S.J., Gierasch, L., and Deisenhofer, J. (1996). The crystal structure of the GroES co-chaperonin at 2.8 A resolution. Nature 379, 37-45. 
Hutchison, K.A., Dittmar, K.D., and Pratt, W.B. (1994). All of the factors required for assembly of the glucocorticoid receptor into a functional heterocomplex with heat shock protein 90 are preassociated in a self-sufficient protein folding structure, a "foldosome". J Biol Chem 269, 27894-27899.

Iwata, A., Christianson, J.C., Bucci, M., Ellerby, L.M., Nukina, N., Forno, L.S., and Kopito, R.R. (2005). Increased susceptibility of cytoplasmic over nuclear polyglutamine aggregates to autophagic degradation. Proc Natl Acad Sci U S A 102, 13135-13140.

Iwata, A., Nagashima, Y., Matsumoto, L., Suzuki, T., Yamanaka, T., Date, H., Deoka, K., Nukina, N., and Tsuji, S. (2009). Intranuclear degradation of polyglutamine aggregates by the ubiquitin-proteasome system. J Biol Chem 284, 9796-9803.

Jana, N.R., Zemskov, E.A., Wang, G., and Nukina, N. (2001). Altered proteasomal function due to the expression of polyglutamine-expanded truncated N-terminal huntingtin induces apoptosis by caspase activation through mitochondrial cytochrome c release. Hum Mol Genet 10, 1049-1059.

Jarosz, D.F., and Lindquist, S. (2010). Hsp90 and environmental stress transform the adaptive value of natural genetic variation. Science 330, 1820-1824.

Joab, I., Radanyi, C., Renoir, M., Buchou, T., Catelli, M.G., Binart, N., Mester, J., and Baulieu, E.E. (1984). Common non-hormone binding component in non-transformed chick oviduct receptors of four steroid hormones. Nature 308, 850-853.

Johnson, B.D., Schumacher, R.J., Ross, E.D., and Toft, D.O. (1998). Hop modulates Hsp70/Hsp90 interactions in protein folding. J Biol Chem 273, 3679-3686.

Johnson, E.S. (2004). Protein modification by SUMO. Annu Rev Biochem 73, 355-382.

Johnston, J.A., Ward, C.L., and Kopito, R.R. (1998). Aggresomes: a cellular response to misfolded proteins. J Cell Biol 143, 1883-1898.

Kabani, M., and Martineau, C.N. (2008). Multiple hsp70 isoforms in the eukaryotic cytosol: mere redundancy or functional specificity? Curr Genomics 9, 338-248.

Kaganovich, D., Kopito, R., and Frydman, J. (2008). Misfolded proteins partition between two distinct quality control compartments. Nature 454, 1088-1095.

Kaiser, C.M., Chang, H.C., Agashe, V.R., Lakshmipathy, S.K., Etchells, S.A., Hayer-Hartl, M., Hartl, F.U., and Barral, J.M. (2006). Real-time observation of trigger factor function on translating ribosomes. Nature 444, 455-460.

Kampinga, H.H. (1993). Thermotolerance in mammalian cells. Protein denaturation and aggregation, and stress proteins. J Cell Sci 104 ( Pt 1), 11-17.

Kampinga, H.H., and Craig, E.A. (2010). The HSP70 chaperone machinery: J proteins as drivers of functional specificity. Nat Rev Mol Cell Biol 11, 579-592.

Karni-Schmidt, O., Zupnick, A., Castillo, M., Ahmed, A., Matos, T., Bouvet, P., Cordon-Cardo, C., and Prives, C. (2008). p53 is localized to a sub-nucleolar compartment after proteasomal inhibition in an energy-dependent manner. J Cell Sci 121, 4098-4105. 
Kauzmann, W. (1959). Some factors in the interpretation of protein denaturation. Adv Protein Chem 14, $1-63$.

Kazemi-Esfarjani, P., and Benzer, S. (2000). Genetic suppression of polyglutamine toxicity in Drosophila. Science 287, 1837-1840.

Kenyon, C. (2005). The plasticity of aging: insights from long-lived mutants. Cell 120, 449-460.

Kenyon, C.J. (2010). The genetics of ageing. Nature 464, 504-512.

Kern, A., Ackermann, B., Clement, A.M., Duerk, H., and Behl, C. (2010). HSF1-controlled and ageassociated chaperone capacity in neurons and muscle cells of C. elegans. PLoS One 5, e8568.

Kerner, M.J., Naylor, D.J., Ishihama, Y., Maier, T., Chang, H.C., Stines, A.P., Georgopoulos, C., Frishman, D., Hayer-Hartl, M., Mann, M., et al. (2005). Proteome-wide analysis of chaperonin-dependent protein folding in Escherichia coli. Cell 122, 209-220.

Kettern, N., Dreiseidler, M., Tawo, R., and Hohfeld, J. (2010). Chaperone-assisted degradation: multiple paths to destruction. Biol Chem 391, 481-489.

Kiaei, M., Kipiani, K., Petri, S., Chen, J., Calingasan, N.Y., and Beal, M.F. (2005). Celastrol blocks neuronal cell death and extends life in transgenic mouse model of amyotrophic lateral sclerosis. Neurodegener Dis 2, 246-254.

Kiffin, R., Christian, C., Knecht, E., and Cuervo, A.M. (2004). Activation of chaperone-mediated autophagy during oxidative stress. Mol Biol Cell 15, 4829-4840.

Kikis, E.A., Gidalevitz, T., and Morimoto, R.I. (2010). Protein homeostasis in models of aging and agerelated conformational disease. Adv Exp Med Biol 694, 138-159.

Kim, P.S., and Baldwin, R.L. (1982). Specific intermediates in the folding reactions of small proteins and the mechanism of protein folding. Annu Rev Biochem 51, 459-489.

Kim, S., Willison, K.R., and Horwich, A.L. (1994). Cystosolic chaperonin subunits have a conserved ATPase domain but diverged polypeptide-binding domains. Trends Biochem Sci 19, 543-548.

Kingston, R.E., Schuetz, T.J., and Larin, Z. (1987). Heat-inducible human factor that binds to a human hsp70 promoter. Mol Cell Biol 7, 1530-1534.

Kisselev, A.F., and Goldberg, A.L. (2001). Proteasome inhibitors: from research tools to drug candidates. Chem Biol 8, 739-758.

Klibanov, S.A., O'Hagan, H.M., and Ljungman, M. (2001). Accumulation of soluble and nucleolarassociated p53 proteins following cellular stress. J Cell Sci 114, 1867-1873.

Klucken, J., Shin, Y., Masliah, E., Hyman, B.T., and McLean, P.J. (2004). Hsp70 Reduces alphaSynuclein Aggregation and Toxicity. J Biol Chem 279, 25497-25502.

Klumpp, M., Baumeister, W., and Essen, L.O. (1997). Structure of the substrate binding domain of the thermosome, an archaeal group II chaperonin. Cell 91, 263-270. 
Komatsu, M., Waguri, S., Chiba, T., Murata, S., Iwata, J., Tanida, I., Ueno, T., Koike, M., Uchiyama, Y., Kominami, E., et al. (2006). Loss of autophagy in the central nervous system causes neurodegeneration in mice. Nature $441,880-884$.

Kopito, R.R. (2000). Aggresomes, inclusion bodies and protein aggregation. Trends Cell Biol 10, 524530.

Kourtis, N., and Tavernarakis, N. (2011). Cellular stress response pathways and ageing: intricate molecular relationships. EMBO J 30, 2520-2531.

Kramer, G., Boehringer, D., Ban, N., and Bukau, B. (2009). The ribosome as a platform for cotranslational processing, folding and targeting of newly synthesized proteins. Nat Struct Mol Biol 16, 589-597.

Kubota, H., Yamamoto, S., Itoh, E., Abe, Y., Nakamura, A., Izumi, Y., Okada, H., Iida, M., Nanjo, H., Itoh, H., et al. (2010). Increased expression of co-chaperone HOP with HSP90 and HSC70 and complex formation in human colonic carcinoma. Cell Stress Chaperones 15, 1003-1011.

Kudo, N., Matsumori, N., Taoka, H., Fujiwara, D., Schreiner, E.P., Wolff, B., Yoshida, M., and Horinouchi, S. (1999). Leptomycin B inactivates CRM1/exportin 1 by covalent modification at a cysteine residue in the central conserved region. Proc Natl Acad Sci U S A 96, 9112-9117.

Kudo, N., Wolff, B., Sekimoto, T., Schreiner, E.P., Yoneda, Y., Yanagida, M., Horinouchi, S., and Yoshida, M. (1998). Leptomycin B inhibition of signal-mediated nuclear export by direct binding to CRM1. Exp Cell Res 242, 540-547.

Kundrat, L., and Regan, L. (2010). Balance between folding and degradation for Hsp90-dependent client proteins: a key role for CHIP. Biochemistry 49, 7428-7438.

la Cour, T., Kiemer, L., Molgaard, A., Gupta, R., Skriver, K., and Brunak, S. (2004). Analysis and prediction of leucine-rich nuclear export signals. Protein Eng Des Sel 17, 527-536.

Laemmli, U.K. (1970). Cleavage of structural proteins during the assembly of the head of bacteriophage T4. Nature 227, 680-685.

Lakshmipathy, S.K., Gupta, R., Pinkert, S., Etchells, S.A., and Hartl, F.U. (2010). Versatility of trigger factor interactions with ribosome-nascent chain complexes. J Biol Chem 285, 27911-27923.

Langer, T., Lu, C., Echols, H., Flanagan, J., Hayer, M.K., and Hartl, F.U. (1992). Successive action of DnaK, DnaJ and GroEL along the pathway of chaperone-mediated protein folding. Nature 356, 683-689.

Lashuel, H.A., Hartley, D., Petre, B.M., Walz, T., and Lansbury, P.T., Jr. (2002). Neurodegenerative disease: amyloid pores from pathogenic mutations. Nature 418, 291.

Laskey, R.A., Honda, B.M., Mills, A.D., and Finch, J.T. (1978). Nucleosomes are assembled by an acidic protein which binds histones and transfers them to DNA. Nature 275, 416-420.

Latonen, L., Moore, H.M., Bai, B., Jaamaa, S., and Laiho, M. (2011). Proteasome inhibitors induce nucleolar aggregation of proteasome target proteins and polyadenylated RNA by altering ubiquitin availability. Oncogene 30, 790-805. 
Levinthal, C., Signer, E.R., and Fetherolf, K. (1962). Reactivation and hybridization of reduced alkaline phosphatase. Proc Natl Acad Sci U S A 48, 1230-1237.

Li, J., Soroka, J., and Buchner, J. (2012). The Hsp90 chaperone machinery: Conformational dynamics and regulation by co-chaperones. Biochim Biophys Acta 1823, 624-635.

Liang, S.T., Xu, Y.C., Dennis, P., and Bremer, H. (2000). mRNA composition and control of bacterial gene expression. J Bacteriol 182, 3037-3044.

Liberek, K., Lewandowska, A., and Zietkiewicz, S. (2008). Chaperones in control of protein disaggregation. EMBO J 27, 328-335.

Liberek, K., Marszalek, J., Ang, D., Georgopoulos, C., and Zylicz, M. (1991a). Escherichia coli DnaJ and GrpE heat shock proteins jointly stimulate ATPase activity of DnaK. Proc Natl Acad Sci U S A 88, 28742878.

Liberek, K., Skowyra, D., Zylicz, M., Johnson, C., and Georgopoulos, C. (1991b). The Escherichia coli DnaK chaperone, the 70-kDa heat shock protein eukaryotic equivalent, changes conformation upon ATP hydrolysis, thus triggering its dissociation from a bound target protein. J Biol Chem 266, 14491-14496.

Lindquist, S. (1986). The heat-shock response. Annu Rev Biochem 55, 1151-1191.

Lindquist, S., and Craig, E.A. (1988). The heat-shock proteins. Annu Rev Genet 22, 631-677.

Lindquist, S.L., and Kelly, J.W. (2011). Chemical and biological approaches for adapting proteostasis to ameliorate protein misfolding and aggregation diseases: progress and prognosis. Cold Spring Harb Perspect Biol 3.

Liu, C., Young, A.L., Starling-Windhof, A., Bracher, A., Saschenbrecker, S., Rao, B.V., Rao, K.V., Berninghausen, O., Mielke, T., Hartl, F.U., et al. (2010). Coupled chaperone action in folding and assembly of hexadecameric Rubisco. Nature 463, 197-202.

Llorca, O., McCormack, E.A., Hynes, G., Grantham, J., Cordell, J., Carrascosa, J.L., Willison, K.R., Fernandez, J.J., and Valpuesta, J.M. (1999). Eukaryotic type II chaperonin CCT interacts with actin through specific subunits. Nature 402, 693-696.

Lum, R., Tkach, J.M., Vierling, E., and Glover, J.R. (2004). Evidence for an unfolding/threading mechanism for protein disaggregation by Saccharomyces cerevisiae Hsp104. J Biol Chem 279, 2913929146.

Lyman, S.K., and Schekman, R. (1997). Binding of secretory precursor polypeptides to a translocon subcomplex is regulated by BiP. Cell $88,85-96$.

Malinovska, L., Kroschwald, S., Munder, M.C., Richter, D., and Alberti, S. (2012). Molecular chaperones and stress-inducible protein sorting factors coordinate the spatio-temporal distribution of protein aggregates. Mol Biol Cell doi: 10.1091/mbc.E12-03-0194

Markesich, D.C., Gajewski, K.M., Nazimiec, M.E., and Beckingham, K. (2000). bicaudal encodes the Drosophila beta NAC homolog, a component of the ribosomal translational machinery*. Development $127,559-572$. 
Marques, C., Guo, W., Pereira, P., Taylor, A., Patterson, C., Evans, P.C., and Shang, F. (2006). The triage of damaged proteins: degradation by the ubiquitin-proteasome pathway or repair by molecular chaperones. FASEB J 20, 741-743.

Martin, J., and Hartl, F.U. (1997). The effect of macromolecular crowding on chaperonin-mediated protein folding. Proc Natl Acad Sci U S A 94, 1107-1112.

Matafora, V., D'Amato, A., Mori, S., Blasi, F., and Bachi, A. (2009). Proteomics analysis of nucleolar SUMO-1 target proteins upon proteasome inhibition. Mol Cell Proteomics 8, 2243-2255.

Matlack, K.E., Misselwitz, B., Plath, K., and Rapoport, T.A. (1999). BiP acts as a molecular ratchet during posttranslational transport of prepro-alpha factor across the ER membrane. Cell 97, 553-564.

Matsui, I., and Harata, K. (2007). Implication for buried polar contacts and ion pairs in hyperthermostable enzymes. FEBS J 274, 4012-4022.

Matthews, B.W., Nicholson, H., and Becktel, W.J. (1987). Enhanced protein thermostability from sitedirected mutations that decrease the entropy of unfolding. Proc Natl Acad Sci U S A 84, 6663-6667.

Mattsson, K., Pokrovskaja, K., Kiss, C., Klein, G., and Szekely, L. (2001). Proteins associated with the promyelocytic leukemia gene product (PML)-containing nuclear body move to the nucleolus upon inhibition of proteasome-dependent protein degradation. Proc Natl Acad Sci U S A 98, 1012-1017.

Maupin-Furlow, J. (2012). Proteasomes and protein conjugation across domains of life. Nat Rev Microbiol 10, 100-111.

Mayer, M.P. (2010). Gymnastics of molecular chaperones. Mol Cell 39, 321-331.

Mayer, M.P., Schroder, H., Rudiger, S., Paal, K., Laufen, T., and Bukau, B. (2000). Multistep mechanism of substrate binding determines chaperone activity of Hsp70. Nat Struct Biol 7, 586-593.

Mayhew, M., da Silva, A.C., Martin, J., Erdjument-Bromage, H., Tempst, P., and Hartl, F.U. (1996). Protein folding in the central cavity of the GroEL-GroES chaperonin complex. Nature 379, 420-426.

McClellan, A.J., Scott, M.D., and Frydman, J. (2005). Folding and quality control of the VHL tumor suppressor proceed through distinct chaperone pathways. Cell 121, 739-748.

McClellan, A.J., Xia, Y., Deutschbauer, A.M., Davis, R.W., Gerstein, M., and Frydman, J. (2007). Diverse cellular functions of the Hsp90 molecular chaperone uncovered using systems approaches. Cell $131,121-135$.

McLaughlin, S.H., Sobott, F., Yao, Z.P., Zhang, W., Nielsen, P.R., Grossmann, J.G., Laue, E.D., Robinson, C.V., and Jackson, S.E. (2006). The co-chaperone p23 arrests the Hsp90 ATPase cycle to trap client proteins. J Mol Biol 356, 746-758.

Meacham, G.C., Patterson, C., Zhang, W., Younger, J.M., and Cyr, D.M. (2001). The Hsc70 cochaperone CHIP targets immature CFTR for proteasomal degradation. Nat Cell Biol 3, 100-105.

Melville, M.W., McClellan, A.J., Meyer, A.S., Darveau, A., and Frydman, J. (2003). The Hsp70 and TRiC/CCT chaperone systems cooperate in vivo to assemble the von Hippel-Lindau tumor suppressor complex. Mol Cell Biol 23, 3141-3151. 
Merksamer, P.I., Trusina, A., and Papa, F.R. (2008). Real-time redox measurements during endoplasmic reticulum stress reveal interlinked protein folding functions. Cell 135, 933-947.

Meyer, A.S., Gillespie, J.R., Walther, D., Millet, I.S., Doniach, S., and Frydman, J. (2003). Closing the folding chamber of the eukaryotic chaperonin requires the transition state of ATP hydrolysis. Cell 113, 369-381.

Meyer, P., Prodromou, C., Liao, C., Hu, B., Mark Roe, S., Vaughan, C.K., Vlasic, I., Panaretou, B., Piper, P.W., and Pearl, L.H. (2004). Structural basis for recruitment of the ATPase activator Aha1 to the Hsp90 chaperone machinery. EMBO J 23, 511-519.

Michels, A.A., Kanon, B., Konings, A.W., Ohtsuka, K., Bensaude, O., and Kampinga, H.H. (1997). Hsp70 and Hsp40 chaperone activities in the cytoplasm and the nucleus of mammalian cells. J Biol Chem 272, 33283-33289.

Michels, A.A., Nguyen, V.T., Konings, A.W., Kampinga, H.H., and Bensaude, O. (1995). Thermostability of a nuclear-targeted luciferase expressed in mammalian cells. Destabilizing influence of the intranuclear microenvironment. Eur J Biochem 234, 382-389.

Miller, J., Arrasate, M., Shaby, B.A., Mitra, S., Masliah, E., and Finkbeiner, S. (2010). Quantitative relationships between huntingtin levels, polyglutamine length, inclusion body formation, and neuronal death provide novel insight into huntington's disease molecular pathogenesis. J Neurosci 30, 1054110550.

Minami, Y., Kimura, Y., Kawasaki, H., Suzuki, K., and Yahara, I. (1994). The carboxy-terminal region of mammalian HSP90 is required for its dimerization and function in vivo. Mol Cell Biol 14, 1459-1464.

Misselwitz, B., Staeck, O., and Rapoport, T.A. (1998). J proteins catalytically activate Hsp70 molecules to trap a wide range of peptide sequences. Mol Cell 2, 593-603.

Mitra, S., Tsvetkov, A.S., and Finkbeiner, S. (2009). Single neuron ubiquitin-proteasome dynamics accompanying inclusion body formation in huntington disease. J Biol Chem 284, 4398-4403.

Mizushima, N., Ohsumi, Y., and Yoshimori, T. (2002). Autophagosome formation in mammalian cells. Cell Struct Funct 27, 421-429.

Mogk, A., Deuerling, E., Vorderwulbecke, S., Vierling, E., and Bukau, B. (2003). Small heat shock proteins, $\mathrm{ClpB}$ and the DnaK system form a functional triade in reversing protein aggregation. Mol Microbiol 50, 585-595.

Mogk, A., Tomoyasu, T., Goloubinoff, P., Rudiger, S., Roder, D., Langen, H., and Bukau, B. (1999). Identification of thermolabile Escherichia coli proteins: prevention and reversion of aggregation by DnaK and ClpB. EMBO J 18, 6934-6949.

Morimoto, R.I. (1993). Cells in stress: transcriptional activation of heat shock genes. Science 259, 14091410.

Morimoto, R.I. (1998). Regulation of the heat shock transcriptional response: cross talk between a family of heat shock factors, molecular chaperones, and negative regulators. Genes Dev 12, 3788-3796. 
Morimoto, R.I. (2008). Proteotoxic stress and inducible chaperone networks in neurodegenerative disease and aging. Genes Dev 22, 1427-1438.

Morimoto, R.I., and Cuervo, A.M. (2009). Protein homeostasis and aging: taking care of proteins from the cradle to the grave. J Gerontol A Biol Sci Med Sci 64, 167-170.

Morley, J.F., and Morimoto, R.I. (2004). Regulation of longevity in Caenorhabditis elegans by heat shock factor and molecular chaperones. Mol Biol Cell 15, 657-664.

Morrow, G., Samson, M., Michaud, S., and Tanguay, R.M. (2004). Overexpression of the small mitochondrial Hsp22 extends Drosophila life span and increases resistance to oxidative stress. FASEB J $18,598-599$.

Mosser, D.D., Duchaine, J., and Massie, B. (1993). The DNA-binding activity of the human heat shock transcription factor is regulated in vivo by hsp70. Mol Cell Biol 13, 5427-5438.

Mossuto, M.F., Dhulesia, A., Devlin, G., Frare, E., Kumita, J.R., de Laureto, P.P., Dumoulin, M., Fontana, A., Dobson, C.M., and Salvatella, X. (2010). The non-core regions of human lysozyme amyloid fibrils influence cytotoxicity. J Mol Biol 402, 783-796.

Mu, T.W., Ong, D.S., Wang, Y.J., Balch, W.E., Yates, J.R., 3rd, Segatori, L., and Kelly, J.W. (2008). Chemical and biological approaches synergize to ameliorate protein-folding diseases. Cell 134, 769-781.

Muchowski, P.J., Schaffar, G., Sittler, A., Wanker, E.E., Hayer-Hartl, M.K., and Hartl, F.U. (2000). Hsp70 and hsp40 chaperones can inhibit self-assembly of polyglutamine proteins into amyloid-like fibrils. Proc Natl Acad Sci U S A 97, 7841-7846.

Muller, W.E., Eckert, A., Kurz, C., Eckert, G.P., and Leuner, K. (2010). Mitochondrial dysfunction: common final pathway in brain aging and Alzheimer's disease--therapeutic aspects. Mol Neurobiol 41, 159-171.

Murata, S., Minami, Y., Minami, M., Chiba, T., and Tanaka, K. (2001). CHIP is a chaperone-dependent E3 ligase that ubiquitylates unfolded protein. EMBO Rep 2, 1133-1138.

Myers, J.K., and Pace, C.N. (1996). Hydrogen bonding stabilizes globular proteins. Biophys J 71, 20332039.

Myung, J., Kim, K.B., and Crews, C.M. (2001). The ubiquitin-proteasome pathway and proteasome inhibitors. Med Res Rev 21, 245-273.

Nadeau, K., Das, A., and Walsh, C.T. (1993). Hsp90 chaperonins possess ATPase activity and bind heat shock transcription factors and peptidyl prolyl isomerases. J Biol Chem 268, 1479-1487.

Nakatsu, T., Ichiyama, S., Hiratake, J., Saldanha, A., Kobashi, N., Sakata, K., and Kato, H. (2006). Structural basis for the spectral difference in luciferase bioluminescence. Nature 440, 372-376.

Naylor, L.H. (1999). Reporter gene technology: the future looks bright. Biochem Pharmacol 58, 749-757.

Neckers, L. (2007). Heat shock protein 90: the cancer chaperone. J Biosci 32, 517-530.

Neef, D.W., Jaeger, A.M., and Thiele, D.J. (2011). Heat shock transcription factor 1 as a therapeutic target in neurodegenerative diseases. Nat Rev Drug Discov 10, 930-944. 
Nekooki-Machida, Y., Kurosawa, M., Nukina, N., Ito, K., Oda, T., and Tanaka, M. (2009). Distinct conformations of in vitro and in vivo amyloids of huntingtin-exon1 show different cytotoxicity. Proc Natl Acad Sci U S A 106, 9679-9684.

Nelson, R.J., Ziegelhoffer, T., Nicolet, C., Werner-Washburne, M., and Craig, E.A. (1992). The translation machinery and $70 \mathrm{kd}$ heat shock protein cooperate in protein synthesis. Cell 71, 97-105.

Netzer, W.J., and Hartl, F.U. (1997). Recombination of protein domains facilitated by co-translational folding in eukaryotes. Nature 388, 343-349.

Nicolet, C.M., and Craig, E.A. (1989). Isolation and characterization of STI1, a stress-inducible gene from Saccharomyces cerevisiae. Mol Cell Biol 9, 3638-3646.

Nimmesgern, E., and Hartl, F.U. (1993). ATP-dependent protein refolding activity in reticulocyte lysate. Evidence for the participation of different chaperone components. FEBS Lett 331, 25-30.

Nollen, E.A., Garcia, S.M., van Haaften, G., Kim, S., Chavez, A., Morimoto, R.I., and Plasterk, R.H. (2004). Genome-wide RNA interference screen identifies previously undescribed regulators of polyglutamine aggregation. Proc Natl Acad Sci U S A 101, 6403-6408.

Nonaka, T., and Hasegawa, M. (2009). A cellular model to monitor proteasome dysfunction by alphasynuclein. Biochemistry 48, 8014-8022.

O'Brien, E.P., Dima, R.I., Brooks, B., and Thirumalai, D. (2007). Interactions between hydrophobic and ionic solutes in aqueous guanidinium chloride and urea solutions: lessons for protein denaturation mechanism. J Am Chem Soc 129, 7346-7353.

Obermann, W.M., Sondermann, H., Russo, A.A., Pavletich, N.P., and Hartl, F.U. (1998). In vivo function of Hsp90 is dependent on ATP binding and ATP hydrolysis. J Cell Biol 143, 901-910.

Oh, E., Becker, A.H., Sandikci, A., Huber, D., Chaba, R., Gloge, F., Nichols, R.J., Typas, A., Gross, C.A., Kramer, G., et al. (2011). Selective ribosome profiling reveals the cotranslational chaperone action of trigger factor in vivo. Cell 147, 1295-1308.

Ohtsuka, K., and Laszlo, A. (1992). The relationship between hsp 70 localization and heat resistance. Exp Cell Res 202, 507-518.

Olsen, A., Vantipalli, M.C., and Lithgow, G.J. (2006). Lifespan extension of Caenorhabditis elegans following repeated mild hormetic heat treatments. Biogerontology 7, 221-230.

Olzscha, H., Schermann, S.M., Woerner, A.C., Pinkert, S., Hecht, M.H., Tartaglia, G.G., Vendruscolo, M., Hayer-Hartl, M., Hartl, F.U., and Vabulas, R.M. (2011). Amyloid-like aggregates sequester numerous metastable proteins with essential cellular functions. Cell 144, 67-78.

Onesto, E., Rusmini, P., Crippa, V., Ferri, N., Zito, A., Galbiati, M., and Poletti, A. (2011). Muscle cells and motoneurons differentially remove mutant SOD1 causing familial amyotrophic lateral sclerosis. J Neurochem 118, 266-280.

Onuchic, J.N., Luthey-Schulten, Z., and Wolynes, P.G. (1997). Theory of protein folding: the energy landscape perspective. Annu Rev Phys Chem 48, 545-600. 
Pahlavani, M.A., Harris, M.D., Moore, S.A., Weindruch, R., and Richardson, A. (1995). The expression of heat shock protein 70 decreases with age in lymphocytes from rats and rhesus monkeys. Exp Cell Res 218, 310-318.

Palleros, D.R., Shi, L., Reid, K.L., and Fink, A.L. (1994). hsp70-protein complexes. Complex stability and conformation of bound substrate protein. J Biol Chem 269, 13107-13114.

Palleros, D.R., Welch, W.J., and Fink, A.L. (1991). Interaction of hsp70 with unfolded proteins: effects of temperature and nucleotides on the kinetics of binding. Proc Natl Acad Sci U S A 88, 5719-5723.

Panaretou, B., Siligardi, G., Meyer, P., Maloney, A., Sullivan, J.K., Singh, S., Millson, S.H., Clarke, P.A., Naaby-Hansen, S., Stein, R., et al. (2002). Activation of the ATPase activity of hsp90 by the stressregulated cochaperone aha1. Mol Cell 10, 1307-1318.

Parker, C.S., and Topol, J. (1984). A Drosophila RNA polymerase II transcription factor binds to the regulatory site of an hsp 70 gene. Cell 37, 273-283.

Parsell, D.A., Kowal, A.S., Singer, M.A., and Lindquist, S. (1994). Protein disaggregation mediated by heat-shock protein Hsp104. Nature 372, 475-478.

Patzelt, H., Rudiger, S., Brehmer, D., Kramer, G., Vorderwulbecke, S., Schaffitzel, E., Waitz, A., Hesterkamp, T., Dong, L., Schneider-Mergener, J., et al. (2001). Binding specificity of Escherichia coli trigger factor. Proc Natl Acad Sci U S A 98, 14244-14249.

Pearl, L.H., and Prodromou, C. (2006). Structure and mechanism of the Hsp90 molecular chaperone machinery. Annu Rev Biochem 75, 271-294.

Pedersen, S. (1984). Escherichia coli ribosomes translate in vivo with variable rate. EMBO J 3, 28952898.

Pelham, H.R. (1982). A regulatory upstream promoter element in the Drosophila hsp 70 heat-shock gene. Cell 30, 517-528.

Pellecchia, M., Montgomery, D.L., Stevens, S.Y., Vander Kooi, C.W., Feng, H.P., Gierasch, L.M., and Zuiderweg, E.R. (2000). Structural insights into substrate binding by the molecular chaperone DnaK. Nat Struct Biol 7, 298-303.

Perisic, O., Xiao, H., and Lis, J.T. (1989). Stable binding of Drosophila heat shock factor to head-to-head and tail-to-tail repeats of a conserved 5 bp recognition unit. Cell 59, 797-806.

Petsko, G.A. (2001). Structural basis of thermostability in hyperthermophilic proteins, or "there's more than one way to skin a cat". Methods Enzymol 334, 469-478.

Pfund, C., Lopez-Hoyo, N., Ziegelhoffer, T., Schilke, B.A., Lopez-Buesa, P., Walter, W.A., Wiedmann, M., and Craig, E.A. (1998). The molecular chaperone Ssb from Saccharomyces cerevisiae is a component of the ribosome-nascent chain complex. EMBO J 17, 3981-3989.

Phillips, P.A., Dudeja, V., McCarroll, J.A., Borja-Cacho, D., Dawra, R.K., Grizzle, W.E., Vickers, S.M., and Saluja, A.K. (2007). Triptolide induces pancreatic cancer cell death via inhibition of heat shock protein 70. Cancer Res 67, 9407-9416. 
Pick, E., Kluger, Y., Giltnane, J.M., Moeder, C., Camp, R.L., Rimm, D.L., and Kluger, H.M. (2007). High HSP90 expression is associated with decreased survival in breast cancer. Cancer Res 67, 2932-2937.

Pickart, C.M. (2000). Ubiquitin in chains. Trends Biochem Sci 25, 544-548.

Pinto, M., Morange, M., and Bensaude, O. (1991). Denaturation of proteins during heat shock. In vivo recovery of solubility and activity of reporter enzymes. J Biol Chem 266, 13941-13946.

Polier, S., Dragovic, Z., Hartl, F.U., and Bracher, A. (2008). Structural basis for the cooperation of Hsp70 and Hsp110 chaperones in protein folding. Cell 133, 1068-1079.

Powers, E.T., Morimoto, R.I., Dillin, A., Kelly, J.W., and Balch, W.E. (2009). Biological and chemical approaches to diseases of proteostasis deficiency. Annu Rev Biochem 78, 959-991.

Powers, M.V., Clarke, P.A., and Workman, P. (2008). Dual targeting of HSC70 and HSP72 inhibits HSP90 function and induces tumor-specific apoptosis. Cancer Cell 14, 250-262.

Prahlad, V., and Morimoto, R.I. (2009). Integrating the stress response: lessons for neurodegenerative diseases from C. elegans. Trends Cell Biol 19, 52-61.

Prasad, R., Kawaguchi, S., and Ng, D.T. (2010). A nucleus-based quality control mechanism for cytosolic proteins. Mol Biol Cell 21, 2117-2127.

Privalov, P.L. (1990). Cold denaturation of proteins. Crit Rev Biochem Mol Biol 25, 281-305.

Prodromou, C., Panaretou, B., Chohan, S., Siligardi, G., O'Brien, R., Ladbury, J.E., Roe, S.M., Piper, P.W., and Pearl, L.H. (2000). The ATPase cycle of Hsp90 drives a molecular 'clamp' via transient dimerization of the N-terminal domains. EMBO J 19, 4383-4392.

Prodromou, C., Roe, S.M., O'Brien, R., Ladbury, J.E., Piper, P.W., and Pearl, L.H. (1997). Identification and structural characterization of the ATP/ADP-binding site in the Hsp90 molecular chaperone. Cell 90, $65-75$.

Prodromou, C., Siligardi, G., O'Brien, R., Woolfson, D.N., Regan, L., Panaretou, B., Ladbury, J.E., Piper, P.W., and Pearl, L.H. (1999). Regulation of Hsp90 ATPase activity by tetratricopeptide repeat (TPR)domain co-chaperones. EMBO J 18, 754-762.

Qiu, X.B., Shao, Y.M., Miao, S., and Wang, L. (2006). The diversity of the DnaJ/Hsp40 family, the crucial partners for Hsp70 chaperones. Cell Mol Life Sci 63, 2560-2570.

Queitsch, C., Sangster, T.A., and Lindquist, S. (2002). Hsp90 as a capacitor of phenotypic variation. Nature 417, 618-624.

Quist, A., Doudevski, I., Lin, H., Azimova, R., Ng, D., Frangione, B., Kagan, B., Ghiso, J., and Lal, R. (2005). Amyloid ion channels: a common structural link for protein-misfolding disease. Proc Natl Acad Sci U S A 102, 10427-10432.

Ravikumar, B., Duden, R., and Rubinsztein, D.C. (2002). Aggregate-prone proteins with polyglutamine and polyalanine expansions are degraded by autophagy. Hum Mol Genet 11, 1107-1117.

Raviol, H., Sadlish, H., Rodriguez, F., Mayer, M.P., and Bukau, B. (2006). Chaperone network in the yeast cytosol: Hsp110 is revealed as an Hsp70 nucleotide exchange factor. EMBO J 25, 2510-2518. 
Richarme, G., and Kohiyama, M. (1993). Specificity of the Escherichia coli chaperone DnaK (70-kDa heat shock protein) for hydrophobic amino acids. J Biol Chem 268, 24074-24077.

Ritossa, F. (1962). New Puffing Pattern Induced by Temperature Shock and Dnp in Drosophila. Experientia 18, 571-573.

Ross, C.A., and Tabrizi, S.J. (2011). Huntington's disease: from molecular pathogenesis to clinical treatment. Lancet Neurol 10, 83-98.

Rosser, M.F., Washburn, E., Muchowski, P.J., Patterson, C., and Cyr, D.M. (2007). Chaperone functions of the E3 ubiquitin ligase CHIP. J Biol Chem 282, 22267-22277.

Rousseau, F., Serrano, L., and Schymkowitz, J.W. (2006). How evolutionary pressure against protein aggregation shaped chaperone specificity. J Mol Biol 355, 1037-1047.

Rubinsztein, D.C. (2006). The roles of intracellular protein-degradation pathways in neurodegeneration. Nature 443, 780-786.

Rudiger, S., Germeroth, L., Schneider-Mergener, J., and Bukau, B. (1997). Substrate specificity of the DnaK chaperone determined by screening cellulose-bound peptide libraries. EMBO J 16, 1501-1507.

Rudiger, S., Schneider-Mergener, J., and Bukau, B. (2001). Its substrate specificity characterizes the DnaJ co-chaperone as a scanning factor for the DnaK chaperone. EMBO J 20, 1042-1050.

Rujano, M.A., Kampinga, H.H., and Salomons, F.A. (2007). Modulation of polyglutamine inclusion formation by the Hsp70 chaperone machine. Exp Cell Res 313, 3568-3578.

Rutherford, S.L., and Lindquist, S. (1998). Hsp90 as a capacitor for morphological evolution. Nature 396, 336-342.

Sakahira, H., Breuer, P., Hayer-Hartl, M.K., and Hartl, F.U. (2002). Molecular chaperones as modulators of polyglutamine protein aggregation and toxicity. Proc Natl Acad Sci U S A 99 Suppl 4, 16412-16418.

Sakikawa, C., Taguchi, H., Makino, Y., and Yoshida, M. (1999). On the maximum size of proteins to stay and fold in the cavity of GroEL underneath GroES. J Biol Chem 274, 21251-21256.

Sambrook, J., Russel, D. W. (2001). Molecular cloning: A Laboratory Manual. Cold Spring Harb Laboratory Press.

Sarge, K.D., Murphy, S.P., and Morimoto, R.I. (1993). Activation of heat shock gene transcription by heat shock factor 1 involves oligomerization, acquisition of DNA-binding activity, and nuclear localization and can occur in the absence of stress. Mol Cell Biol 13, 1392-1407.

Saschenbrecker, S., Bracher, A., Rao, K.V., Rao, B.V., Hartl, F.U., and Hayer-Hartl, M. (2007). Structure and function of RbcX, an assembly chaperone for hexadecameric Rubisco. Cell 129, 1189-1200.

Satyal, S.H., Schmidt, E., Kitagawa, K., Sondheimer, N., Lindquist, S., Kramer, J.M., and Morimoto, R.I. (2000). Polyglutamine aggregates alter protein folding homeostasis in Caenorhabditis elegans. Proc Natl Acad Sci U S A 97, 5750-5755. 
Saudou, F., Finkbeiner, S., Devys, D., and Greenberg, M.E. (1998). Huntingtin acts in the nucleus to induce apoptosis but death does not correlate with the formation of intranuclear inclusions. Cell 95, 5566.

Scherzinger, E., Lurz, R., Turmaine, M., Mangiarini, L., Hollenbach, B., Hasenbank, R., Bates, G.P., Davies, S.W., Lehrach, H., and Wanker, E.E. (1997). Huntingtin-encoded polyglutamine expansions form amyloid-like protein aggregates in vitro and in vivo. Cell 90, 549-558.

Scheufler, C., Brinker, A., Bourenkov, G., Pegoraro, S., Moroder, L., Bartunik, H., Hartl, F.U., and Moarefi, I. (2000). Structure of TPR domain-peptide complexes: critical elements in the assembly of the Hsp70-Hsp90 multichaperone machine. Cell 101, 199-210.

Schmid, A.B., Lagleder, S., Grawert, M.A., Rohl, A., Hagn, F., Wandinger, S.K., Cox, M.B., Demmer, O., Richter, K., Groll, M., et al. (2012). The architecture of functional modules in the Hsp90 cochaperone Sti1/Hop. EMBO J 31, 1506-1517.

Schneider, C., Sepp-Lorenzino, L., Nimmesgern, E., Ouerfelli, O., Danishefsky, S., Rosen, N., and Hartl, F.U. (1996). Pharmacologic shifting of a balance between protein refolding and degradation mediated by Hsp90. Proc Natl Acad Sci U S A 93, 14536-14541.

Schroder, H., Langer, T., Hartl, F.U., and Bukau, B. (1993). DnaK, DnaJ and GrpE form a cellular chaperone machinery capable of repairing heat-induced protein damage. EMBO J 12, 4137-4144.

Schuh, S., Yonemoto, W., Brugge, J., Bauer, V.J., Riehl, R.M., Sullivan, W.P., and Toft, D.O. (1985). A 90,000-dalton binding protein common to both steroid receptors and the Rous sarcoma virus transforming protein, pp60v-src. J Biol Chem 260, 14292-14296.

Schumacher, R.J., Hansen, W.J., Freeman, B.C., Alnemri, E., Litwack, G., and Toft, D.O. (1996). Cooperative action of Hsp70, Hsp90, and DnaJ proteins in protein renaturation. Biochemistry 35, 1488914898.

Scott, M.D., and Frydman, J. (2003). Aberrant protein folding as the molecular basis of cancer. Methods Mol Biol 232, 67-76.

Seidel, K., Meister, M., Dugbartey, G.J., Zijlstra, M.P., Vinet, J., Brunt, E.R., van Leeuwen, F.W., Rub, U., Kampinga, H.H., and den Dunnen, W.F. (2011). Cellular protein quality control and the evolution of aggregates in SCA3. Neuropathol Appl Neurobiol doi: 10.1111/j.1365-2990.2011.01220.x.

Seliger, H.H., and Mc, E.W. (1960). Spectral emission and quantum yield of firefly bioluminescence. Arch Biochem Biophys 88, 136-141.

Sharma, K., Vabulas, R.M., Macek, B., Pinkert, S., Cox, J., Mann, M., and Hartl, F.U. (2012). Quantitative proteomics reveals that Hsp90 inhibition preferentially targets kinases and the DNA damage response. Mol Cell Proteomics 11.

Sharma, S.K., De los Rios, P., Christen, P., Lustig, A., and Goloubinoff, P. (2010). The kinetic parameters and energy cost of the Hsp70 chaperone as a polypeptide unfoldase. Nat Chem Biol 6, 914920.

Sharp, S., and Workman, P. (2006). Inhibitors of the HSP90 molecular chaperone: current status. Adv Cancer Res 95, 323-348. 
Sherf, B.A., and Wood, K.V. (1994). Firefly luciferase engineered for improved genetic reporting. Promega Notes Magazine 49.

Shi, Y., Mosser, D.D., and Morimoto, R.I. (1998). Molecular chaperones as HSF1-specific transcriptional repressors. Genes Dev 12, 654-666.

Shorter, J. (2011). The mammalian disaggregase machinery: Hsp110 synergizes with Hsp70 and Hsp40 to catalyze protein disaggregation and reactivation in a cell-free system. PLoS One 6, e26319.

Siegert, R., Leroux, M.R., Scheufler, C., Hartl, F.U., and Moarefi, I. (2000). Structure of the molecular chaperone prefoldin: unique interaction of multiple coiled coil tentacles with unfolded proteins. Cell 103, 621-632.

Sieradzan, K.A., Mechan, A.O., Jones, L., Wanker, E.E., Nukina, N., and Mann, D.M. (1999). Huntington's disease intranuclear inclusions contain truncated, ubiquitinated huntingtin protein. Exp Neurol 156, 92-99.

Silva, M.C., Fox, S., Beam, M., Thakkar, H., Amaral, M.D., and Morimoto, R.I. (2011). A genetic screening strategy identifies novel regulators of the proteostasis network. PLoS Genet 7, e1002438.

Sittler, A., Lurz, R., Lueder, G., Priller, J., Lehrach, H., Hayer-Hartl, M.K., Hartl, F.U., and Wanker, E.E. (2001). Geldanamycin activates a heat shock response and inhibits huntingtin aggregation in a cell culture model of Huntington's disease. Hum Mol Genet 10, 1307-1315.

Smith, D.F. (1993). Dynamics of heat shock protein 90-progesterone receptor binding and the disactivation loop model for steroid receptor complexes. Mol Endocrinol 7, 1418-1429.

Smith, D.F., Stensgard, B.A., Welch, W.J., and Toft, D.O. (1992). Assembly of progesterone receptor with heat shock proteins and receptor activation are ATP mediated events. J Biol Chem 267, 1350-1356.

Smith, D.F., Sullivan, W.P., Marion, T.N., Zaitsu, K., Madden, B., McCormick, D.J., and Toft, D.O. (1993). Identification of a 60-kilodalton stress-related protein, p60, which interacts with hsp90 and hsp70. Mol Cell Biol 13, 869-876.

Smith, D.M., Kafri, G., Cheng, Y., Ng, D., Walz, T., and Goldberg, A.L. (2005). ATP binding to PAN or the 26S ATPases causes association with the 20S proteasome, gate opening, and translocation of unfolded proteins. Mol Cell 20, 687-698.

Snell, R.G., MacMillan, J.C., Cheadle, J.P., Fenton, I., Lazarou, L.P., Davies, P., MacDonald, M.E., Gusella, J.F., Harper, P.S., and Shaw, D.J. (1993). Relationship between trinucleotide repeat expansion and phenotypic variation in Huntington's disease. Nat Genet 4, 393-397.

Sorger, P.K., and Nelson, H.C. (1989). Trimerization of a yeast transcriptional activator via a coiled-coil motif. Cell 59, 807-813.

Soto, C. (2003). Unfolding the role of protein misfolding in neurodegenerative diseases. Nat Rev Neurosci 4, 49-60.

Southworth, D.R., and Agard, D.A. (2011). Client-loading conformation of the Hsp90 molecular chaperone revealed in the cryo-EM structure of the human Hsp90:Hop complex. Mol Cell 42, 771-781. 
Spiess, C., Miller, E.J., McClellan, A.J., and Frydman, J. (2006). Identification of the TRiC/CCT substrate binding sites uncovers the function of subunit diversity in eukaryotic chaperonins. Mol Cell 24, 25-37.

Stankiewicz, M., Nikolay, R., Rybin, V., and Mayer, M.P. (2010). CHIP participates in protein triage decisions by preferentially ubiquitinating Hsp70-bound substrates. FEBS J 277, 3353-3367.

Stebbins, C.E., Russo, A.A., Schneider, C., Rosen, N., Hartl, F.U., and Pavletich, N.P. (1997). Crystal structure of an Hsp90-geldanamycin complex: targeting of a protein chaperone by an antitumor agent. Cell 89, 239-250.

Steffan, J.S., Agrawal, N., Pallos, J., Rockabrand, E., Trotman, L.C., Slepko, N., Illes, K., Lukacsovich, T., Zhu, Y.Z., Cattaneo, E., et al. (2004). SUMO modification of Huntingtin and Huntington's disease pathology. Science 304, 100-104.

Stickle, D.F., Presta, L.G., Dill, K.A., and Rose, G.D. (1992). Hydrogen bonding in globular proteins. J Mol Biol 226, 1143-1159.

Stirling, P.C., Bakhoum, S.F., Feigl, A.B., and Leroux, M.R. (2006). Convergent evolution of clamp-like binding sites in diverse chaperones. Nat Struct Mol Biol 13, 865-870.

Suhr, S.T., Senut, M.C., Whitelegge, J.P., Faull, K.F., Cuizon, D.B., and Gage, F.H. (2001). Identities of sequestered proteins in aggregates from cells with induced polyglutamine expression. J Cell Biol 153, 283-294.

Sullivan, W., Stensgard, B., Caucutt, G., Bartha, B., McMahon, N., Alnemri, E.S., Litwack, G., and Toft, D. (1997). Nucleotides and two functional states of hsp90. J Biol Chem 272, 8007-8012.

Sulston, J.E., and Horvitz, H.R. (1977). Post-embryonic cell lineages of the nematode, Caenorhabditis elegans. Dev Biol 56, 110-156.

Szabo, A., Korszun, R., Hartl, F.U., and Flanagan, J. (1996). A zinc finger-like domain of the molecular chaperone DnaJ is involved in binding to denatured protein substrates. EMBO J 15, 408-417.

Szabo, A., Langer, T., Schroder, H., Flanagan, J., Bukau, B., and Hartl, F.U. (1994). The ATP hydrolysisdependent reaction cycle of the Escherichia coli Hsp70 system DnaK, DnaJ, and GrpE. Proc Natl Acad Sci U S A 91, 10345-10349.

Taipale, M., Jarosz, D.F., and Lindquist, S. (2010). HSP90 at the hub of protein homeostasis: emerging mechanistic insights. Nat Rev Mol Cell Biol 11, 515-528.

Takahashi, T., Kikuchi, S., Katada, S., Nagai, Y., Nishizawa, M., and Onodera, O. (2008). Soluble polyglutamine oligomers formed prior to inclusion body formation are cytotoxic. Hum Mol Genet 17, 345-356.

Takayama, S., Bimston, D.N., Matsuzawa, S., Freeman, B.C., Aime-Sempe, C., Xie, Z., Morimoto, R.I., and Reed, J.C. (1997). BAG-1 modulates the chaperone activity of Hsp70/Hsc70. EMBO J 16, 48874896.

Tanford, C. (1970). Protein denaturation. C. Theoretical models for the mechanism of denaturation. Adv Protein Chem 24, 1-95. 
Tang, Y.C., Chang, H.C., Roeben, A., Wischnewski, D., Wischnewski, N., Kerner, M.J., Hartl, F.U., and Hayer-Hartl, M. (2006). Structural features of the GroEL-GroES nano-cage required for rapid folding of encapsulated protein. Cell 125, 903-914.

Tartaglia, G.G., Pechmann, S., Dobson, C.M., and Vendruscolo, M. (2007). Life on the edge: a link between gene expression levels and aggregation rates of human proteins. Trends Biochem Sci 32, 204206.

Taylor, R.C., and Dillin, A. (2011). Aging as an event of proteostasis collapse. Cold Spring Harb Perspect Biol 3.

Teng, S., Luo, H., and Wang, L. (2012). Predicting protein sumoylation sites from sequence features. Amino Acids 43, 447-455.

Terada, K., and Mori, M. (2000). Human DnaJ homologs dj2 and dj3, and bag-1 are positive cochaperones of hsc70. J Biol Chem 275, 24728-24734.

Teter, S.A., Houry, W.A., Ang, D., Tradler, T., Rockabrand, D., Fischer, G., Blum, P., Georgopoulos, C., and Hartl, F.U. (1999). Polypeptide flux through bacterial Hsp70: DnaK cooperates with trigger factor in chaperoning nascent chains. Cell 97, 755-765.

The_Huntington's_Disease_Collaborative_Research_Group (1993). A novel gene containing a trinucleotide repeat that is expanded and unstable on Huntington's disease chromosomes. Cell 72, 971983.

Thompson, J.F., Hayes, L.S., and Lloyd, D.B. (1991). Modulation of firefly luciferase stability and impact on studies of gene regulation. Gene 103, 171-177.

Thrower, J.S., Hoffman, L., Rechsteiner, M., and Pickart, C.M. (2000). Recognition of the polyubiquitin proteolytic signal. EMBO J 19, 94-102.

Thulasiraman, V., and Matts, R.L. (1996). Effect of geldanamycin on the kinetics of chaperone-mediated renaturation of firefly luciferase in rabbit reticulocyte lysate. Biochemistry 35, 13443-13450.

Thulasiraman, V., Yang, C.F., and Frydman, J. (1999). In vivo newly translated polypeptides are sequestered in a protected folding environment. EMBO J 18, 85-95.

Tian, G., Vainberg, I.E., Tap, W.D., Lewis, S.A., and Cowan, N.J. (1995). Specificity in chaperoninmediated protein folding. Nature 375, 250-253.

Tomko, R.J., Jr., and Hochstrasser, M. (2011). Order of the proteasomal ATPases and eukaryotic proteasome assembly. Cell Biochem Biophys 60, 13-20.

Tonoki, A., Kuranaga, E., Tomioka, T., Hamazaki, J., Murata, S., Tanaka, K., and Miura, M. (2009). Genetic evidence linking age-dependent attenuation of the $26 \mathrm{~S}$ proteasome with the aging process. Mol Cell Biol 29, 1095-1106.

Trinklein, N.D., Murray, J.I., Hartman, S.J., Botstein, D., and Myers, R.M. (2004). The role of heat shock transcription factor 1 in the genome-wide regulation of the mammalian heat shock response. Mol Biol Cell 15, 1254-1261. 
Trott, A., West, J.D., Klaic, L., Westerheide, S.D., Silverman, R.B., Morimoto, R.I., and Morano, K.A. (2008). Activation of heat shock and antioxidant responses by the natural product celastrol: transcriptional signatures of a thiol-targeted molecule. Mol Biol Cell 19, 1104-1112.

Tyedmers, J., Mogk, A., and Bukau, B. (2010). Cellular strategies for controlling protein aggregation. Nat Rev Mol Cell Biol 11, 777-788.

Urushitani, M., Kurisu, J., Tateno, M., Hatakeyama, S., Nakayama, K., Kato, S., and Takahashi, R. (2004). CHIP promotes proteasomal degradation of familial ALS-linked mutant SOD1 by ubiquitinating Hsp/Hsc70. J Neurochem 90, 231-244.

Vainberg, I.E., Lewis, S.A., Rommelaere, H., Ampe, C., Vandekerckhove, J., Klein, H.L., and Cowan, N.J. (1998). Prefoldin, a chaperone that delivers unfolded proteins to cytosolic chaperonin. Cell 93, 863873.

van den Berg, B., Wain, R., Dobson, C.M., and Ellis, R.J. (2000). Macromolecular crowding perturbs protein refolding kinetics: implications for folding inside the cell. EMBO J 19, 3870-3875.

Vavouri, T., Semple, J.I., Garcia-Verdugo, R., and Lehner, B. (2009). Intrinsic protein disorder and interaction promiscuity are widely associated with dosage sensitivity. Cell 138, 198-208.

Velazquez, J.M., and Lindquist, S. (1984). hsp70: nuclear concentration during environmental stress and cytoplasmic storage during recovery. Cell $36,655-662$.

Velten, M., Villoutreix, B.O., and Ladjimi, M.M. (2000). Quaternary structure of the HSC70 cochaperone HIP. Biochemistry 39, 307-315.

Venkatraman, P., Wetzel, R., Tanaka, M., Nukina, N., and Goldberg, A.L. (2004). Eukaryotic proteasomes cannot digest polyglutamine sequences and release them during degradation of polyglutamine-containing proteins. Mol Cell 14, 95-104.

Vieille, C., and Zeikus, G.J. (2001). Hyperthermophilic enzymes: sources, uses, and molecular mechanisms for thermostability. Microbiol Mol Biol Rev 65, 1-43.

Viitanen, P.V., Gatenby, A.A., and Lorimer, G.H. (1992). Purified chaperonin 60 (groEL) interacts with the nonnative states of a multitude of Escherichia coli proteins. Protein Sci 1, 363-369.

Voges, D., Zwickl, P., and Baumeister, W. (1999). The 26S proteasome: a molecular machine designed for controlled proteolysis. Annu Rev Biochem 68, 1015-1068.

Vos, M.J., Hageman, J., Carra, S., and Kampinga, H.H. (2008). Structural and functional diversities between members of the human HSPB, HSPH, HSPA, and DNAJ chaperone families. Biochemistry 47, 7001-7011.

Walerych, D., Olszewski, M.B., Gutkowska, M., Helwak, A., Zylicz, M., and Zylicz, A. (2009). Hsp70 molecular chaperones are required to support p53 tumor suppressor activity under stress conditions. Oncogene 28, 4284-4294.

Walter, P., and Ron, D. (2011). The unfolded protein response: from stress pathway to homeostatic regulation. Science 334, 1081-1086. 
Wang, J., Gines, S., MacDonald, M.E., and Gusella, J.F. (2005). Reversal of a full-length mutant huntingtin neuronal cell phenotype by chemical inhibitors of polyglutamine-mediated aggregation. BMC Neurosci 6,1 .

Warrick, J.M., Chan, H.Y., Gray-Board, G.L., Chai, Y., Paulson, H.L., and Bonini, N.M. (1999). Suppression of polyglutamine-mediated neurodegeneration in Drosophila by the molecular chaperone HSP70. Nat Genet 23, 425-428.

Webb, J.L., Ravikumar, B., Atkins, J., Skepper, J.N., and Rubinsztein, D.C. (2003). Alpha-Synuclein is degraded by both autophagy and the proteasome. J Biol Chem 278, 25009-25013.

Wegele, H., Wandinger, S.K., Schmid, A.B., Reinstein, J., and Buchner, J. (2006). Substrate transfer from the chaperone Hsp70 to Hsp90. J Mol Biol 356, 802-811.

Welch, W.J., and Feramisco, J.R. (1984). Nuclear and nucleolar localization of the 72,000-dalton heat shock protein in heat-shocked mammalian cells. J Biol Chem 259, 4501-4513.

Westerheide, S.D., Bosman, J.D., Mbadugha, B.N., Kawahara, T.L., Matsumoto, G., Kim, S., Gu, W., Devlin, J.P., Silverman, R.B., and Morimoto, R.I. (2004). Celastrols as inducers of the heat shock response and cytoprotection. J Biol Chem 279, 56053-56060.

Westerheide, S.D., Kawahara, T.L., Orton, K., and Morimoto, R.I. (2006). Triptolide, an inhibitor of the human heat shock response that enhances stress-induced cell death. J Biol Chem 281, 9616-9622.

Westerheide, S.D., and Morimoto, R.I. (2005). Heat shock response modulators as therapeutic tools for diseases of protein conformation. J Biol Chem 280, 33097-33100.

Westwood, J.T., Clos, J., and $\mathrm{Wu}$, C. (1991). Stress-induced oligomerization and chromosomal relocalization of heat-shock factor. Nature 353, 822-827.

Whitesell, L., and Lindquist, S.L. (2005). HSP90 and the chaperoning of cancer. Nat Rev Cancer 5, 761772.

Wiech, H., Buchner, J., Zimmermann, R., and Jakob, U. (1992). Hsp90 chaperones protein folding in vitro. Nature 358, 169-170.

Winklhofer, K.F., Tatzelt, J., and Haass, C. (2008). The two faces of protein misfolding: gain- and lossof-function in neurodegenerative diseases. EMBO J 27, 336-349.

Wisen, S., Bertelsen, E.B., Thompson, A.D., Patury, S., Ung, P., Chang, L., Evans, C.G., Walter, G.M., Wipf, P., Carlson, H.A., et al. (2010). Binding of a small molecule at a protein-protein interface regulates the chaperone activity of hsp70-hsp40. ACS Chem Biol 5, 611-622.

Wisen, S., and Gestwicki, J.E. (2008). Identification of small molecules that modify the protein folding activity of heat shock protein 70. Anal Biochem 374, 371-377.

Wolf, D.H., and Hilt, W. (2004). The proteasome: a proteolytic nanomachine of cell regulation and waste disposal. Biochim Biophys Acta 1695, 19-31.

Wong, E., and Cuervo, A.M. (2010). Integration of clearance mechanisms: the proteasome and autophagy. Cold Spring Harb Perspect Biol 2, a006734. 
$\mathrm{Wu}, \mathrm{C}$. (1984). Activating protein factor binds in vitro to upstream control sequences in heat shock gene chromatin. Nature 311, 81-84.

Wyttenbach, A., Carmichael, J., Swartz, J., Furlong, R.A., Narain, Y., Rankin, J., and Rubinsztein, D.C. (2000). Effects of heat shock, heat shock protein 40 (HDJ-2), and proteasome inhibition on protein aggregation in cellular models of Huntington's disease. Proc Natl Acad Sci U S A 97, 2898-2903.

Xu, Z., Horwich, A.L., and Sigler, P.B. (1997). The crystal structure of the asymmetric GroEL-GroES(ADP)7 chaperonin complex. Nature 388, 741-750.

Yaffe, M.B., Farr, G.W., Miklos, D., Horwich, A.L., Sternlicht, M.L., and Sternlicht, H. (1992). TCP1 complex is a molecular chaperone in tubulin biogenesis. Nature 358, 245-248.

Yam, A.Y., Xia, Y., Lin, H.T., Burlingame, A., Gerstein, M., and Frydman, J. (2008). Defining the TRiC/CCT interactome links chaperonin function to stabilization of newly made proteins with complex topologies. Nat Struct Mol Biol 15, 1255-1262.

Yamamoto, A., Lucas, J.J., and Hen, R. (2000). Reversal of neuropathology and motor dysfunction in a conditional model of Huntington's disease. Cell 101, 57-66.

Yang, W., Dunlap, J.R., Andrews, R.B., and Wetzel, R. (2002). Aggregated polyglutamine peptides delivered to nuclei are toxic to mammalian cells. Hum Mol Genet 11, 2905-2917.

Yonehara, M., Minami, Y., Kawata, Y., Nagai, J., and Yahara, I. (1996). Heat-induced chaperone activity of HSP90. J Biol Chem 271, 2641-2645.

Young, J.C., and Hartl, F.U. (2000). Polypeptide release by Hsp90 involves ATP hydrolysis and is enhanced by the co-chaperone p23. EMBO J 19, 5930-5940.

Young, J.C., Hoogenraad, N.J., and Hartl, F.U. (2003). Molecular chaperones Hsp90 and Hsp70 deliver preproteins to the mitochondrial import receptor Tom70. Cell 112, 41-50.

Young, J.C., Obermann, W.M., and Hartl, F.U. (1998). Specific binding of tetratricopeptide repeat proteins to the C-terminal 12-kDa domain of hsp90. J Biol Chem 273, 18007-18010.

Zhang, C., and Cuervo, A.M. (2008). Restoration of chaperone-mediated autophagy in aging liver improves cellular maintenance and hepatic function. Nat Med 14, 959-965.

Zhang, S., Binari, R., Zhou, R., and Perrimon, N. (2010). A genomewide RNA interference screen for modifiers of aggregates formation by mutant Huntingtin in Drosophila. Genetics 184, 1165-1179.

Zhang, Y.Q., and Sarge, K.D. (2007). Celastrol inhibits polyglutamine aggregation and toxicity though induction of the heat shock response. J Mol Med (Berl) 85, 1421-1428.

Zhu, X., Zhao, X., Burkholder, W.F., Gragerov, A., Ogata, C.M., Gottesman, M.E., and Hendrickson, W.A. (1996). Structural analysis of substrate binding by the molecular chaperone DnaK. Science 272, 1606-1614.

Zietkiewicz, S., Krzewska, J., and Liberek, K. (2004). Successive and synergistic action of the Hsp70 and Hsp100 chaperones in protein disaggregation. J Biol Chem 279, 44376-44383. 
Zijlstra, M.P., Rujano, M.A., Van Waarde, M.A., Vis, E., Brunt, E.R., and Kampinga, H.H. (2010). Levels of DNAJB family members (HSP40) correlate with disease onset in patients with spinocerebellar ataxia type 3. Eur J Neurosci 32, 760-770.

Zimmerman, S.B., and Trach, S.O. (1991). Estimation of macromolecule concentrations and excluded volume effects for the cytoplasm of Escherichia coli. J Mol Biol 222, 599-620.

Zou, J., Guo, Y., Guettouche, T., Smith, D.F., and Voellmy, R. (1998). Repression of heat shock transcription factor HSF1 activation by HSP90 (HSP90 complex) that forms a stress-sensitive complex with HSF1. Cell 94, 471-480.

Zourlidou, A., Gidalevitz, T., Kristiansen, M., Landles, C., Woodman, B., Wells, D.J., Latchman, D.S., de Belleroche, J., Tabrizi, S.J., Morimoto, R.I., et al. (2007). Hsp27 overexpression in the R6/2 mouse model of Huntington's disease: chronic neurodegeneration does not induce Hsp27 activation. Hum Mol Genet $16,1078-1090$.

Zuccato, C., Valenza, M., and Cattaneo, E. (2010). Molecular mechanisms and potential therapeutical targets in Huntington's disease. Physiol Rev 90, 905-981.

Zwanzig, R., Szabo, A., and Bagchi, B. (1992). Levinthal's paradox. Proc Natl Acad Sci U S A 89, 20-22. 


\section{Appendices}

\section{VIII.1. Abbreviations}

17-AAG 17-allylamino-17-demethoxygeldnamycin

AD Alzheimer's Disease

ADP Adenosine 5'-diphosphate

ALS Amyotrophic Lateral Sclerosis

ANS 1-anilino-8-naphthalenesulfonate

ATP Adenosine 5'-triphosphate

CASA Chaperone Assisted Selective Autophagy

C. elegans Caenorhabditis elegans

CCT Chaperonin Containing TCP-1

CDKs Cyclin Dependent kinases

CFTR Cystic Fibrosis Transmembrane Conductance Regulator

CHIP Carboxy terminus of Hsp70 Interacting Protein

CHX Cycloheximide

CMA Chaperone Mediated Autophagy

CPY Carboxypeptidase Y enzyme

CTD Carboxyl terminal Domain

DMSO Dimethyl Sulphoxide

DNA Deoxyribo Nucleic Acid

DnaJ Bacterial Hsp40 Co-chaperone

DnaK Bacterial Hsp70 Chaperone

DUBs Deubiquitylating Enzymes

E. coli Escherichia coli

EGFP Enhanced Green Fluorescent Protein

Fluc wild-type Firefly Luciferase

FlucDM Fluc Double Mutant (luciferase with point mutations R188Q and R261Q)

FlucSM Fluc Single Mutant (luciferase with point mutation R188Q)

g Acceleration of Gravity, $9.81 \mathrm{~m} / \mathrm{sec}^{2}$

$\mathrm{GdmCl}$ Guanidinium Chloride

GroEL Bacterial Hsp60 Chaperonin 


\begin{tabular}{|c|c|}
\hline GroES & Bacterial Hsp10 Chaperonin \\
\hline GrpE & Bacterial Nucleotide Exchange factor of DnaK \\
\hline $\mathrm{h}$ & Hour \\
\hline HD & Huntington's Disease \\
\hline HIP & Hsp70 Interacting Protein \\
\hline HOP & Hsp70-Hsp90 Organizing Protein \\
\hline Hsc70 & Heat Shock Cognate 70 \\
\hline HSEs & Heat Shock Elements \\
\hline HSF1 & Heat Shock Factor 1 \\
\hline Hsp110 & Heat Shock Protein 110 \\
\hline Hsp27 & Heat Shock Protein 27 \\
\hline Hsp40 & Heat Shock Protein 40 \\
\hline Hsp70 & Heat Shock Protein 70 \\
\hline Hsp90 & Heat Shock Protein 90 \\
\hline HSPs & Heat Shock Proteins \\
\hline HtpG & High temperature protein $\mathrm{G}$ \\
\hline $\mathrm{Htt}$ & Huntingtin \\
\hline IBs & Inclusion Bodies \\
\hline IIS & Insulin/IGF1 like Signaling \\
\hline IPOD & Insoluble Protein Deposit \\
\hline JUNQ & Juxtanuclear Quality Control \\
\hline LB & Luria Bertani \\
\hline LMB & Leptomycin B \\
\hline MD & Middle Domain \\
\hline mRNA & Messenger RNA \\
\hline MTOC & Microtubule Organizing Centre \\
\hline NAC & Nascent Chain Associated Complex \\
\hline NEF & Nucleotide Exchange Factor \\
\hline NES & Nuclear Export Signal \\
\hline NLS & Nuclear Localization Signal \\
\hline NPM1 & Nucleophosmin-1. Also called B23 \\
\hline NTD & Nucleotide Domain \\
\hline O.D. & Optical Density \\
\hline
\end{tabular}


PBT 2-phenyl benzothiazole

PC Pharmacological Chaperone

PCR Polymerase Chain Reaction

PD Parkinson's Disease

PDB Protein Data Bank

PFD Prefoldin

PML Promyelocytic Leukemia Gene Product

PN Proteostasis Network

PolyQ Polyglutamine

PR Proteostasis regulators

RAC Ribosome Associated Chaperones

Rluc Renilla reniformis Luciferase

RNA Ribo Nucleic Acid

RRL Rabbit Reticulocyte Lysate

RT-PCR Reverse Transcriptase PCR

Rubisco Ribulose-1,5-bisphosphate Carboxylase Oxygenase

S. cerevisiae Saccharomyces cerevisiae

SBMA Spinal Bulbar Muscular Atrophy

SDS Sodium Dodecyl Sulfate

SOD1 Superoxide Dismutase 1

SUMO Small Ubiquitin like Modifier

TF Trigger Factor

TPR Tetratricopeptide Repeat

TRAP TNFR Associated Protein

TRiC TCP-1 Ring Complex

UPR Unfolded Protein Response

UPS Ubiquitin Proteasome System

VHL Von Hippel-Lindau Protein 


\section{VIII.2. Curriculum Vitae}

Name

Birth Date

Birth Place

Nationality

Dissertation

Aug. 2008 - Present

\section{Education}

May 2007 - May 2008

May 2006 - May 2008

May 2003 - May 2006
Rajat Gupta

December 03, 1984

New Delhi, India

Indian
Ph.D. dissertation under the supervision of Prof. Dr. F. Ulrich Hartl in the Department of Cellular Biochemistry, Max Planck Institute of Biochemistry, Martinsried.

Dissertation title: Firefly luciferase mutants as sensors of proteome stress

Master thesis under the supervision of Prof. Dr. Dulal Panda at Indian Institute of Technology-Bombay, Mumbai, India.

Title: Interaction of tau protein with microtubules: implications in neuronal disorders.

Master of Science in Biotechnology from Indian Institute of Technology-Bombay, Mumbai, India.

Bachelor of Science (Biochemistry Honors) from Deshbandhu College, University of Delhi, New Delhi, India.

\section{Higher Education}

May 2001 - May 2003

Mother Teresa Public School, New Delhi, India. 